\title{
Defluorodearomatization: a Photocatalytic Birch-like Reduction that Enables C-C Bond Formation and Provides Access to Unnatural Cannabinoids
}

Jon I. Day ${ }^{\alpha, \nu}$, Sascha Grotjahn ${ }^{\beta, \nu}$, Sameera Senaweera ${ }^{\delta}$, Burkhard Koenig ${ }^{\beta}$, Jimmie D. Weaver III ${ }^{\alpha *}$

${ }^{\alpha}$ Department of Chemistry, Oklahoma State University, 107, Physical Science, 74078 Stillwater, Oklahoma, United States

${ }^{\beta}$ Institut für Organische Chemie, Universität Regensburg, Universitätstrasse 31, 93053 Regensburg, Germany

${ }^{\delta}$ Center for Drug Design, College of Pharmacy, University of Minnesota, 7-158 Phillips Wangensteen Building, 516

Delaware Street SE, Minneapolis, MN 55455

jimmie.weaver@okstate.edu

Supporting Information 


\section{Table of Contents}

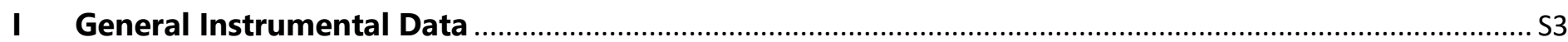

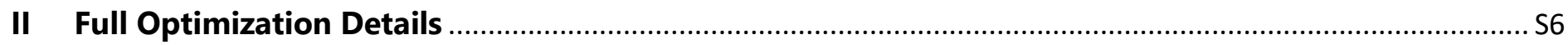

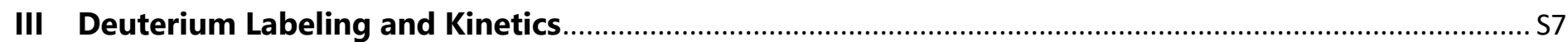

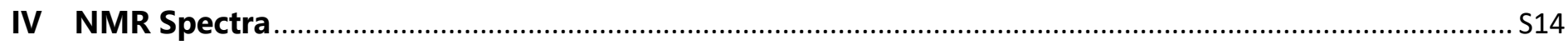

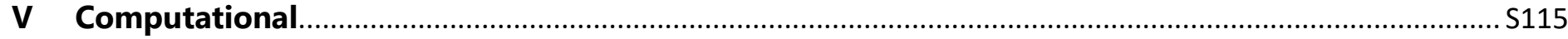

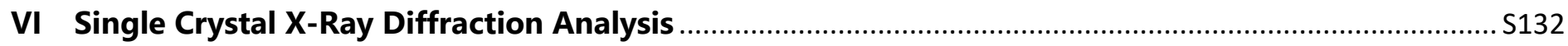

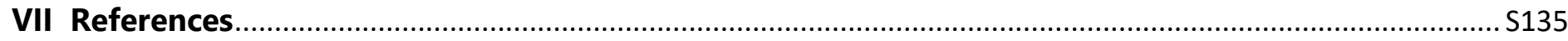




\section{General Instrumental Data}

\section{Reaction Setup for Photo-Birch Arylation}

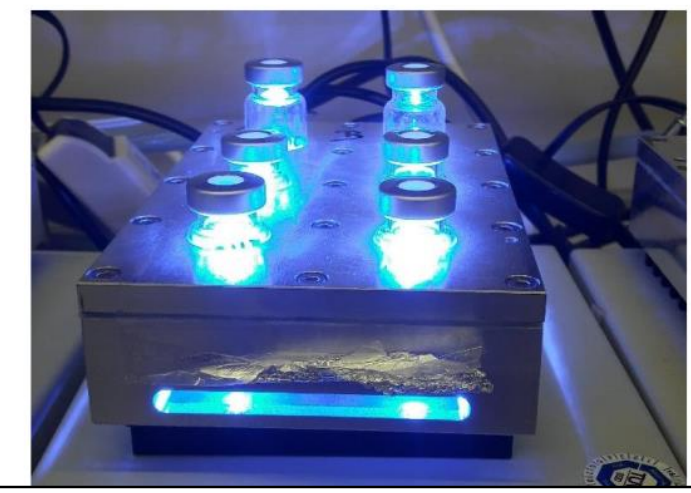

Reactions for substrate screening were conducted in sealed glass vials by irradiation with OSRAM Oslon SSL 80 royal-blue LEDs $(\lambda=455 \mathrm{~nm}( \pm 15$ $\mathrm{nm}$ ), $3.5 \mathrm{~V}, 700 \mathrm{~mA}$ ), or in NMR tubes (as below)

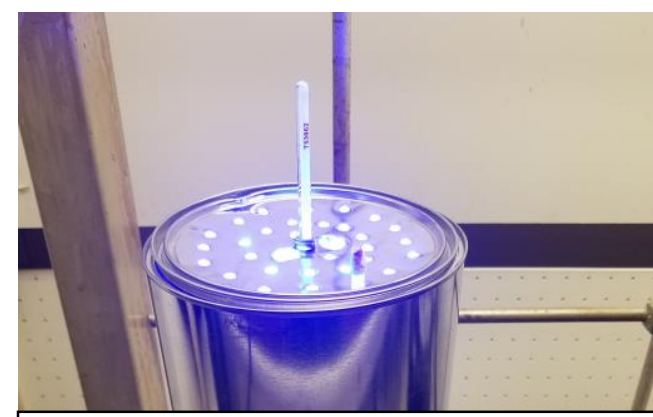

Room temperature high intensity light bath (NMR scale) - lights are Solid Apollo Blue Waterproof 5050 72W LED Strip Light 465 nm SKU: SA-LS-BL-5050-300-IP67-24V
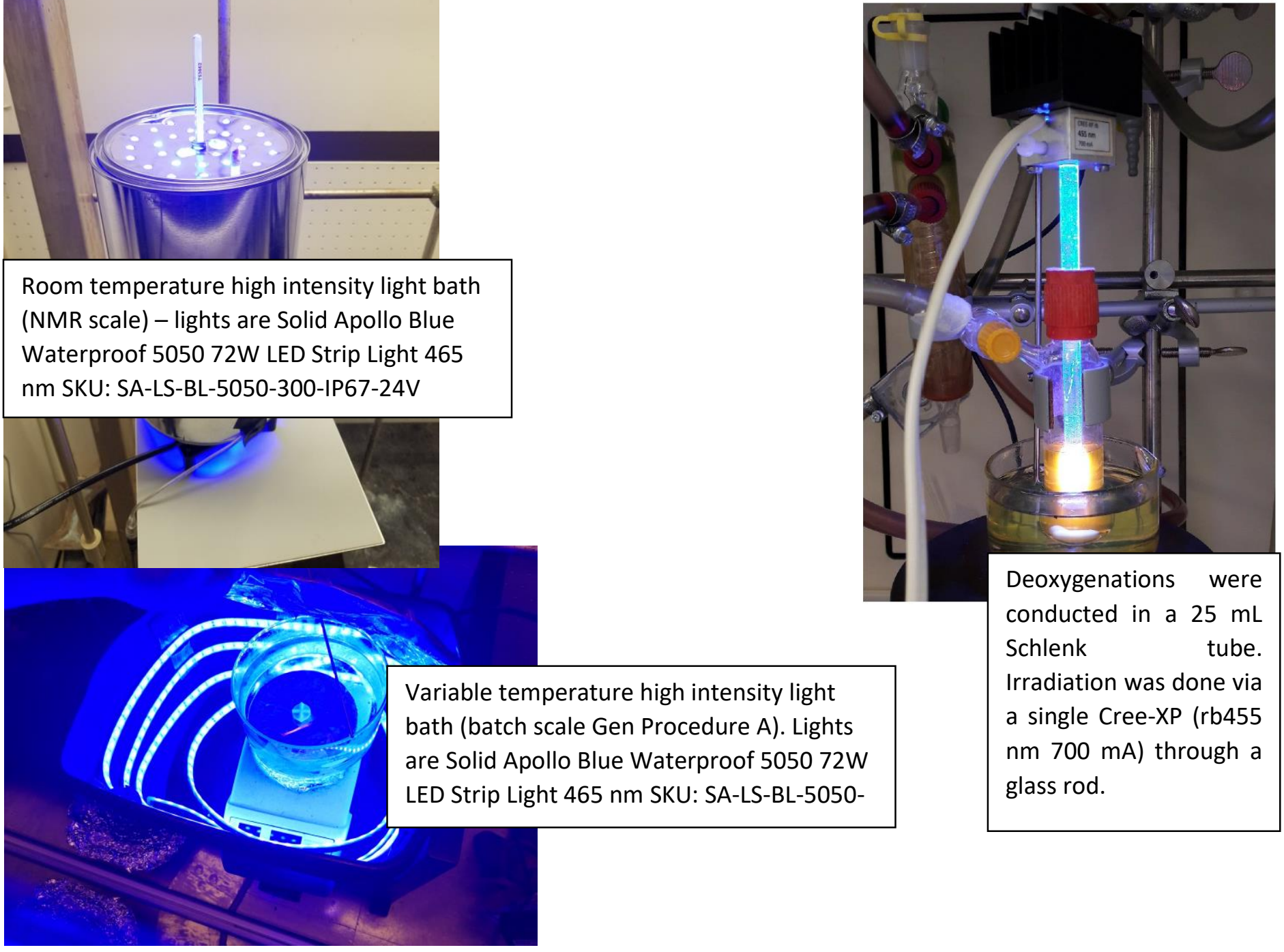
Large scale Tauchschachtreactor
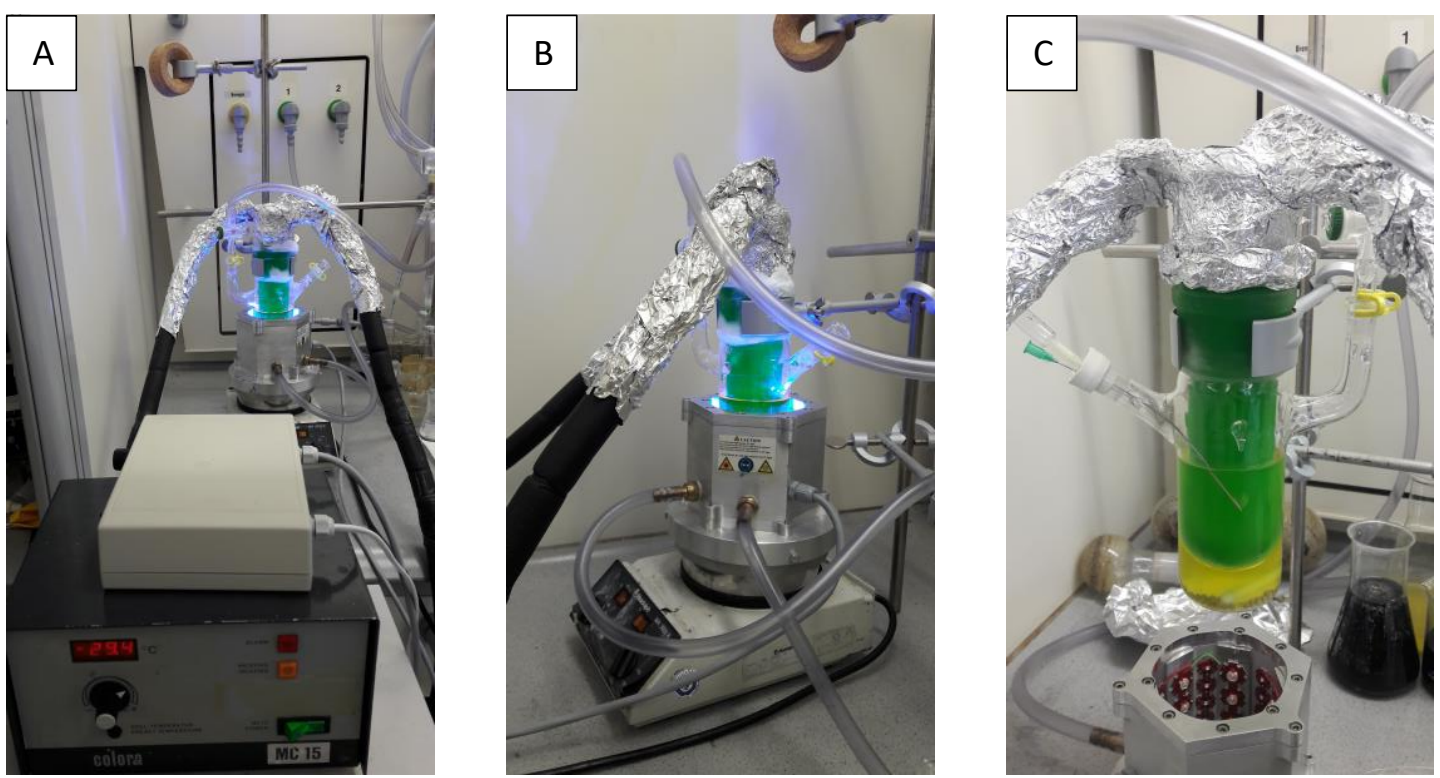

Large scale reactions (General Procedure B) were conducted in a $120 \mathrm{~mL}$ vessel Tauchschachtreactor, irradiated by 24 OSRAM Oslon SSL 80 royal-blue LEDs $(\lambda=455 \mathrm{~nm}( \pm 15 \mathrm{~nm}), 3.5 \mathrm{~V}, 700 \mathrm{~mA})$. A: full setup in action, with reaction vessel inside reactor (back) and thermostat (front); B: the reaction vessel is placed on a magnetic stirrer. The LEDs are water cooled; C: reaction vessel outside the reactor. The reaction mixture is degassed by purging with nitrogen through a cannula. The reaction mixture is cooled by a cooling finger connected to the thermostat.

Strong cooling by the thermostat was necessary to dissipate the heat from the LEDs. A thermostat temperature of $-30^{\circ} \mathrm{C}$ was used to keep the reaction mixture at approximately $10^{\circ} \mathrm{C}$.

No change in yield and product distribution was observed between the different reactor types. For reaction conditions see general procedure $B$ in the experimental part. 

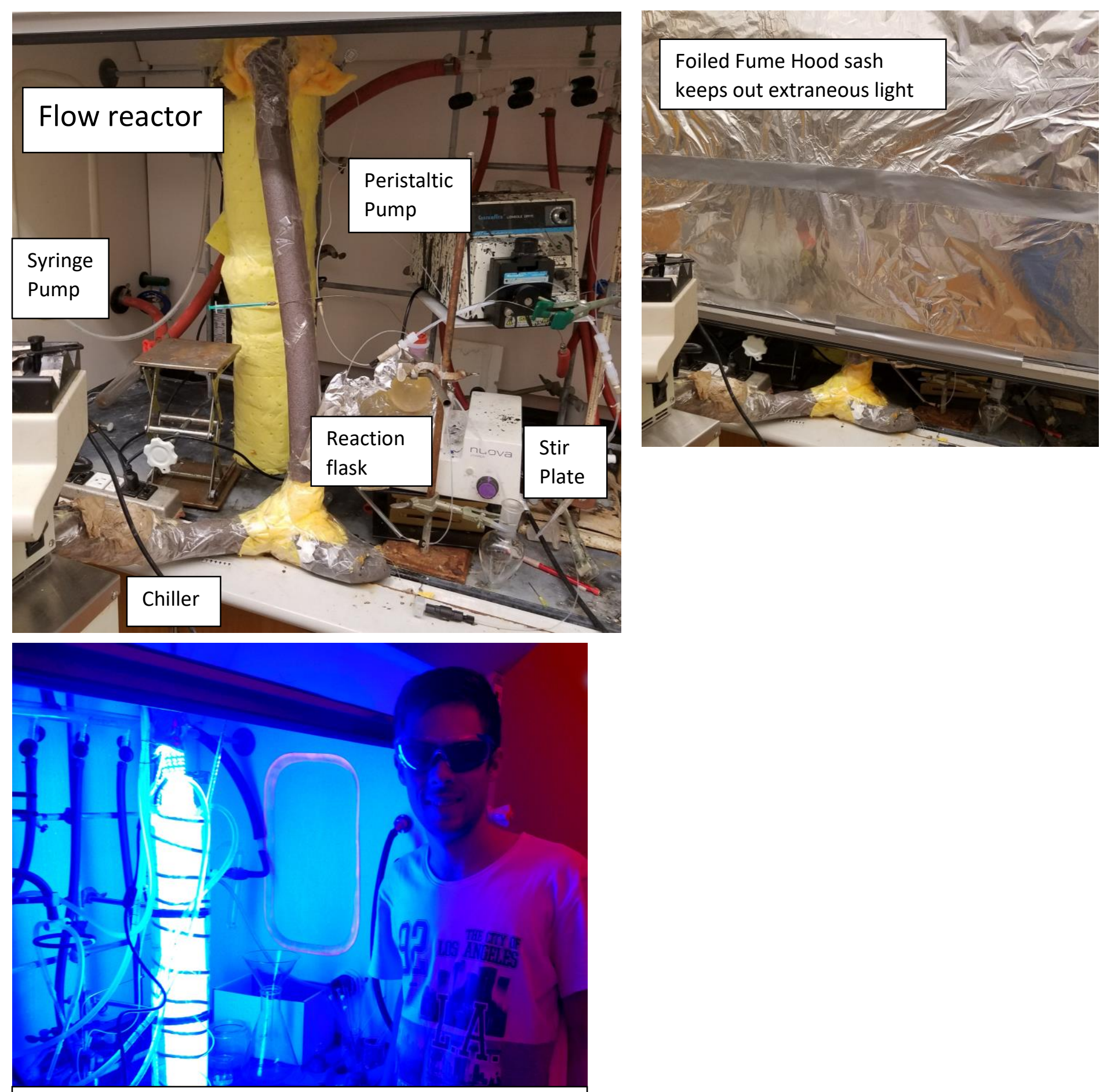

Sascha demonstrating LED intensity with insulation removed. Lights are Solid Apollo Blue Eco 3528 Double Row 96W LED Strip Light SKU: SA-LS-BL-3528-1200-24V 


\section{Full Optimization Details}

Table 1: Optimization of the Reaction Conditions<smiles>N#Cc1c(F)c(F)c(F)c(F)c1F</smiles>

(Ar-F)<smiles>[R]c1ccc(C(=O)OC)cc1</smiles>

(Ar-H)<smiles>[R]C1=CC(c2c(F)c(C)cc(C)c2F)C(C(=O)OC)=CC1</smiles>

(PDT)<smiles>N#Cc1c(F)c(F)cc(F)c1F</smiles>

(HDF)

Equiv. Equiv. Equiv. $0.25 \mathrm{Mol} \% \quad$ conc. Temp. Time

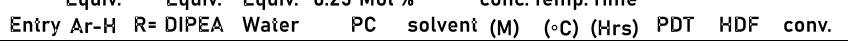

\begin{tabular}{|c|c|c|c|c|c|c|c|c|c|c|c|c|c|}
\hline \multirow{9}{*}{ 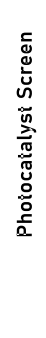 } & 1 & 2.6 & $\mathrm{Me}$ & 1.5 & 0 & $\mathrm{PCl}$ & $\mathrm{ACN}$ & 0.1 & 0 & 18 & 4 & 6 & 20 \\
\hline & 2 & 2.6 & $\mathrm{Me}$ & 1.5 & 0 & PC2 & $\mathrm{ACN}$ & 0.1 & 0 & 18 & 1 & 2 & 7 \\
\hline & 3 & 2.6 & $\mathrm{Me}$ & 1.5 & 0 & PC3 & $\mathrm{ACN}$ & 0.1 & 0 & 18 & 16 & 52 & 100 \\
\hline & 4 & 2.6 & $\mathrm{Me}$ & 1.5 & 0 & PC4 & $\mathrm{ACN}$ & 0.1 & 0 & 18 & 15 & 32 & 73 \\
\hline & 5 & 2.6 & $\mathrm{Me}$ & 1.5 & 0 & PC5 & $\mathrm{ACN}$ & 0.1 & 0 & 18 & 16 & 27 & 90 \\
\hline & 6 & 2.6 & $\mathrm{Me}$ & 1.5 & 0 & PC6 & $\mathrm{ACN}$ & 0.1 & 0 & 18 & 9 & 20 & 51 \\
\hline & 7 & 2.6 & $\mathrm{Me}$ & 1.5 & 0 & PC7 & $\mathrm{ACN}$ & 0.1 & 0 & 18 & 7 & 27 & 50 \\
\hline & 8 & 2.6 & $\mathrm{Me}$ & 1.5 & 0 & PC8 & $\mathrm{ACN}$ & 0.1 & 0 & 18 & 3 & 7 & 25 \\
\hline & 9 & 2.6 & $\mathrm{Me}$ & 1.5 & 0 & PC9 & $\mathrm{ACN}$ & 0.1 & 25 & 18 & 7 & 20 & 60 \\
\hline
\end{tabular}
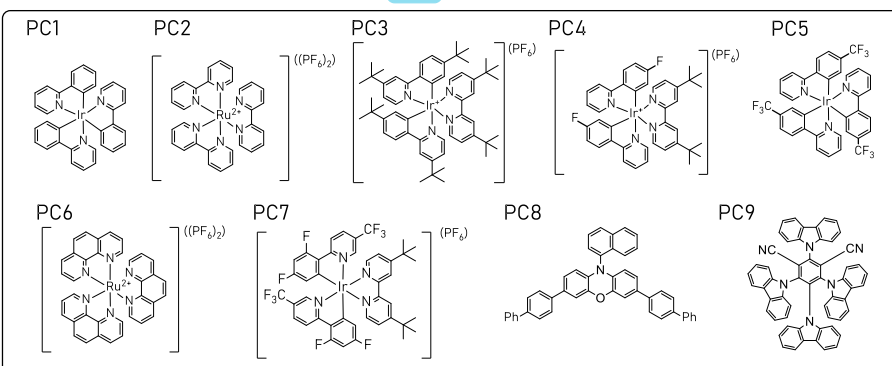

Equiv. Equiv. Equiv. Mol \% conc. Temp.Time

Enùry Ar-H R= DIPEA Waier PC3 solvenî (M) (०C) (Hrs) PDT HDF conv.

\begin{tabular}{|c|c|c|c|c|c|c|c|c|c|c|c|c|}
\hline 9 & 3.0 & $\mathrm{Me}$ & 1.1 & 0.0 & 0.25 & $\mathrm{ACN}$ & 0.1 & 0 & 18 & 8.6 & 24.8 & 48.7 \\
\hline 10 & 3.0 & $\mathrm{Me}$ & 1.1 & 1 & 0.25 & $\mathrm{ACN}$ & 0.1 & 0 & 18 & 17.0 & 46.1 & 93.4 \\
\hline 11 & 3.0 & $\mathrm{Me}$ & 1.1 & 5 & 0.25 & $\mathrm{ACN}$ & 0.1 & 0 & 18 & 21.3 & 54.8 & 100 \\
\hline 12 & 3.0 & $\mathrm{Me}$ & 1.1 & 15 & 0.25 & $\mathrm{ACN}$ & 0.1 & 0 & 18 & 22.0 & 48.5 & 100 \\
\hline 13 & 3.0 & $\mathrm{Me}$ & 1.1 & 20 & 0.25 & $\mathrm{ACN}$ & 0.1 & 0 & 18 & 19.1 & 36.6 & 100 \\
\hline 14 & 3.0 & $\mathrm{Me}$ & 1.1 & 100 & 0.25 & $\mathrm{ACN}$ & 0.1 & 0 & 18 & 17.4 & 24.6 & 100 \\
\hline
\end{tabular}

\begin{tabular}{|c|c|c|c|c|c|c|c|c|c|c|c|c|c|}
\hline & \multicolumn{3}{|c|}{ Equiv. } & \multirow{2}{*}{$\begin{array}{l}1.1 \text { Equiv } \\
\text { Reduciant }\end{array}$} & \multicolumn{3}{|c|}{ Equiv. Mol \% } & \multicolumn{3}{|c|}{ conc. Temp.Time } & \multirow[b]{2}{*}{ PDT } & \multirow{2}{*}{\multicolumn{2}{|c|}{ HDF conv }} \\
\hline & Eniry & Ar-H & $R=$ & & Water & PC3 & solvenî & (M) & $(\circ \mathrm{C})$ & (Hrs) & & & \\
\hline & 15 & 2.6 & $\mathrm{Me}$ & DIPEA & 15 & 0.25 & $\mathrm{ACN}$ & 0.1 & $\mathrm{rt}$ & 18 & 30.3 & 37.2 & 100 \\
\hline & 16 & 2.6 & $\mathrm{Me}$ & TMP & 15 & 0.25 & $\mathrm{ACN}$ & 0.1 & $\mathrm{rt}$ & 18 & 1.3 & 1.7 & 30.3 \\
\hline & 17 & 2.6 & $\mathrm{Me}$ & TEA & 15 & 0.25 & $\mathrm{ACN}$ & 0.1 & $\mathrm{rt}$ & 18 & 3.7 & 8.6 & 97.6 \\
\hline & 18 & 2.6 & $\mathrm{Me}$ & $\begin{array}{c}\text { Hantzsch } \\
\text { Ester }\end{array}$ & 15 & 0.25 & $\mathrm{ACN}$ & 0.1 & $\mathrm{rt}$ & 18 & 0.9 & 1.7 & 34.1 \\
\hline & 19 & 2.6 & $\mathrm{Me}$ & $\mathrm{Bu}_{3} \mathrm{~N}$ & 15 & 0.25 & $\mathrm{ACN}$ & 0.1 & $\mathrm{rt}$ & 18 & 31.6 & 31.7 & 100 \\
\hline & 20 & 2.6 & $\mathrm{Me}$ & DABCO & 15 & 0.25 & $\mathrm{ACN}$ & 0.1 & $\mathrm{rt}$ & 18 & $\operatorname{tr}$ & 7.5 & 57.5 \\
\hline
\end{tabular}

Conversions/yields determined against internal standard

Conducted in $1 \mathrm{~mL}$ solvent NMR tube.

Equiv. Equiv. Equiv. Mol\% conc. Temp.Time Eniry Ar-H R= DIPEA Water PC3 solveni $(M) \quad\left({ }^{\circ} \mathrm{C}\right)(\mathrm{Hrs})$ PDT HDF conv.

\begin{tabular}{|c|c|c|c|c|c|c|c|c|c|c|c|c|c|}
\hline \multirow{7}{*}{ 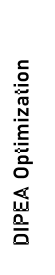 } & 21 & 2.6 & $\mathrm{Me}$ & 0.0 & 10 & 0.25 & $\mathrm{ACN}$ & 0.1 & 0 & 18 & 0.0 & 0.0 & 0.0 \\
\hline & 22 & 2.6 & $\mathrm{Me}$ & 0.5 & 10 & 0.25 & $\mathrm{ACN}$ & 0.1 & 0 & 18 & 13.8 & 28 & 57.0 \\
\hline & 23 & 2.6 & $\mathrm{Me}$ & 1.0 & 10 & 0.25 & $\mathrm{ACN}$ & 0.1 & 0 & 18 & 21.0 & 50.7 & 100 \\
\hline & 24 & 2.6 & $\mathrm{Me}$ & 1.2 & 10 & 0.25 & $\mathrm{ACN}$ & 0.1 & 0 & 18 & 18.8 & 48.3 & 100 \\
\hline & 25 & 2.6 & $\mathrm{Me}$ & 1.5 & 10 & 0.25 & $\mathrm{ACN}$ & 0.1 & 0 & 18 & 15.5 & 39.3 & 100 \\
\hline & 26 & 2.6 & $\mathrm{Me}$ & 3.0 & 10 & 0.25 & $\mathrm{ACN}$ & 0.1 & 0 & 18 & 8.5 & 29.5 & 100 \\
\hline & 27 & 2.6 & $\mathrm{Me}$ & 10.0 & 10 & 0.25 & $\mathrm{ACN}$ & 0.1 & 0 & 18 & 3.0 & 7.6 & 100 \\
\hline \multirow{5}{*}{ 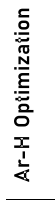 } & 28 & 0.0 & $\mathrm{Me}$ & 1.2 & 10 & 0.25 & $\mathrm{ACN}$ & 0.1 & 0 & 18 & 0.0 & 56.7 & 100 \\
\hline & 29 & 1.0 & $\mathrm{Me}$ & 1.2 & 10 & 0.25 & $\mathrm{ACN}$ & 0.1 & 0 & 18 & 10.5 & 46.5 & 100 \\
\hline & 30 & 2.0 & $\mathrm{Me}$ & 1.2 & 10 & 0.25 & $\mathrm{ACN}$ & 0.1 & 0 & 18 & 17.2 & 43.0 & 100 \\
\hline & 31 & 3.0 & $\mathrm{Me}$ & 1.2 & 10 & 0.25 & $\mathrm{ACN}$ & 0.1 & 0 & 18 & 18.0 & 42.0 & 100 \\
\hline & 32 & 5.0 & $\mathrm{Me}$ & 1.2 & 10 & 0.25 & $\mathrm{ACN}$ & 0.1 & 0 & 18 & 18.3 & 36.3 & 100 \\
\hline \multirow{5}{*}{ 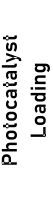 } & 33 & 3.0 & $\mathrm{Me}$ & 1.1 & 10 & 1.00 & $\mathrm{ACN}$ & 0.1 & 0 & 18 & 13.0 & 28.5 & 87.5 \\
\hline & 34 & 3.0 & $\mathrm{Me}$ & 1.1 & 10 & 0.50 & $\mathrm{ACN}$ & 0.1 & 0 & 18 & 14.5 & 26.5 & 100 \\
\hline & 35 & 3.0 & $\mathrm{Me}$ & 1.1 & 10 & 0.25 & $\mathrm{ACN}$ & 0.1 & 0 & 18 & 17.5 & 34.5 & 100 \\
\hline & 36 & 3.0 & $\mathrm{Me}$ & 1.1 & 10 & 0.10 & $\mathrm{ACN}$ & 0.1 & 0 & 18 & 9.5 & 20 & 75.6 \\
\hline & 37 & 3.0 & $\mathrm{Me}$ & 1.1 & 10 & 0.01 & $\mathrm{ACN}$ & 0.1 & 0 & 18 & tr & $\mathrm{tr}$ & 15 \\
\hline \multirow{9}{*}{ 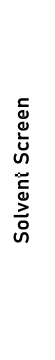 } & 38 & 3.0 & $\mathrm{Me}$ & 1.1 & 15 & 0.25 & $\mathrm{Et}_{2} \mathrm{O}$ & 0.1 & 0 & 18 & 0 & 45.8 & 100 \\
\hline & 39 & 3.0 & $\mathrm{Me}$ & 1.1 & 15 & 0.25 & THF & 0.1 & 0 & 18 & 0 & 44.9 & 100 \\
\hline & 40 & 3.0 & $\mathrm{Me}$ & 1.1 & 15 & 0.25 & $\mathrm{ACN}$ & 0.1 & 0 & 18 & 30.4 & 63.8 & 100 \\
\hline & 41 & 3.0 & $\mathrm{Me}$ & 1.1 & 15 & 0.25 & MTBE & 0.1 & 0 & 18 & 0 & 47.2 & 100 \\
\hline & 42 & 3.0 & $\mathrm{Me}$ & 1.1 & 15 & 0.25 & DME & 0.1 & 0 & 18 & 0 & 47.0 & 100 \\
\hline & 43 & 3.0 & $\mathrm{Me}$ & 1.1 & 15 & 0.25 & toluene & 0.1 & 0 & 18 & 0 & 28.8 & 61.7 \\
\hline & 44 & 3.0 & $\mathrm{Me}$ & 1.1 & 15 & 0.25 & DMSO & 0.1 & 0 & 18 & 0 & 31.9 & 47.4 \\
\hline & 45 & 3.0 & $\mathrm{Me}$ & 1.1 & 15 & 0.25 & DMF & 0.1 & 0 & 18 & 11.8 & 23.5 & 46.7 \\
\hline & 46 & 3.0 & $\mathrm{Me}$ & 1.1 & 15 & 0.25 & DCM & 0.1 & 0 & 18 & 16.1 & 34.8 & 94.7 \\
\hline \multirow{4}{*}{ 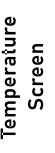 } & 47 & 3.0 & $\mathrm{Me}$ & 1.1 & 15 & 0.25 & $\mathrm{ACN}$ & 0.1 & -2 & 1.0 & 25.3 & 48.1 & 100 \\
\hline & 48 & 3.0 & $\mathrm{Me}$ & 1.1 & 15 & 0.25 & $\mathrm{ACN}$ & 0.1 & $\mathrm{rt}$ & 1.0 & 26.3 & 59.8 & 100 \\
\hline & 49 & 3.0 & $\mathrm{Me}$ & 1.1 & 15 & 0.25 & $\mathrm{ACN}$ & 0.1 & 50 & 1.0 & 28.7 & 66.1 & 100 \\
\hline & 50 & 3.0 & $\mathrm{Me}$ & 1.1 & 15 & 0.25 & $\mathrm{ACN}$ & 0.1 & 75 & 1.0 & 20.8 & 59.1 & 100 \\
\hline \multirow{7}{*}{ 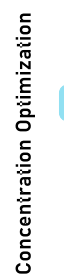 } & 51 & 3.0 & $\mathrm{tBu}$ & 1.1 & 15 & 0.25 & $\mathrm{ACN}$ & 1.00 & $r t$ & 1.25 & 24.8 & 49.6 & 82.8 \\
\hline & 52 & 3.0 & $\mathrm{tBu}$ & 1.1 & 15 & 0.25 & $\mathrm{ACN}$ & 0.50 & $\mathrm{rt}$ & 1.25 & 33.8 & 57.1 & 100 \\
\hline & 53 & 3.0 & $\mathrm{tBu}$ & 1.1 & 15 & 0.25 & $\mathrm{ACN}$ & 0.20 & $r t$ & 1.25 & 37.1 & 58.1 & 100 \\
\hline & 54 & 3.0 & $\mathrm{tBu}$ & 1.1 & 15 & 0.25 & $\mathrm{ACN}$ & 0.10 & $\mathrm{rt}$ & 1.25 & 34.9 & 57.2 & 100 \\
\hline & 55 & 3.0 & $\mathrm{tBu}$ & 1.1 & 15 & 0.25 & $\mathrm{ACN}$ & 0.05 & $\mathrm{rt}$ & 1.25 & 33.8 & 59.8 & 100 \\
\hline & 56 & 3.0 & $\mathrm{tBu}$ & 1.1 & 15 & 0.25 & $\mathrm{ACN}$ & 0.02 & $r t$ & 1.25 & 27.1 & 58.8 & 100 \\
\hline & 57 & 3.0 & $\mathrm{tBu}$ & 1.1 & 15 & 0.25 & $\mathrm{ACN}$ & 0.01 & $\mathrm{rt}$ & 1.25 & 20.2 & 60.5 & 92.9 \\
\hline
\end{tabular}




\section{Deuterium Labeling and Kinetics}<smiles>N#Cc1c(F)c(F)c(F)c(F)c1F</smiles>

$0.1 \mathrm{mmol}$

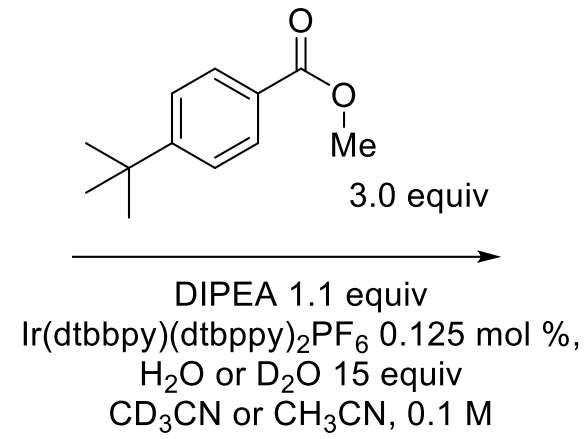<smiles>COC(=O)C1=CCC(C(C)(C)C)=CC1c1c(F)c(F)c(C#N)c(F)c1F</smiles>

General procedure D was followed.

\begin{tabular}{|c|c|c|c|c|}
\hline time Temp. & Conc & solvent & Water & Comment \\
\hline \multirow[t]{4}{*}{$18 \mathrm{~h}$} & $0.1 \mathrm{M}$ & $\mathrm{CD}_{3} \mathrm{CN}$ & $\mathrm{D}_{2} \mathrm{O}$ & D incorporated in product $-\mathrm{m} / \mathrm{z} 368$ \\
\hline & & $\mathrm{CH}_{3} \mathrm{CN}$ & $\mathrm{D}_{2} \mathrm{O}$ & D incorporated in product $-\mathrm{m} / \mathrm{z} 368$ \\
\hline & & $\mathrm{CH}_{3} \mathrm{CN}$ & $\mathrm{H}_{2} \mathrm{O}$ & no deuterium incorporation \\
\hline & & $\mathrm{CD}_{3} \mathrm{CN}$ & $\mathrm{H}_{2} \mathrm{O}$ & no deuterium incorporation \\
\hline
\end{tabular}

It could be that the DIPEA is serving to provide a proton/deuteron source, ${ }^{56}$ but it seems untenable to invoke a side reaction in which a catalytic cycle would incorporate deuterium into the amine when the reactions are complete in such a short time frame, and in the absence of a proton shuttle.<smiles>COC(=O)c1ccc(C)cc1</smiles>

$4.0 \mathrm{mmol}$ DIPEA 1.1 equiv. $\operatorname{Ir}\left(\right.$ dibbpy)(díbppy) ${ }_{2} \mathrm{PF}_{6} 0.125 \mathrm{~mol} \%$ Aceioniťrile $0.2 \mathrm{M}$ $\mathrm{D}_{2} \mathrm{O} 15$ equiv.<smiles>[2H]C1C=C(C(=O)OC)C(c2c(F)c(F)c(C#N)c(F)c2F)C=C1C</smiles>

(22)<smiles>COC(=O)C1=CCC(C)=CC1(C)c1c(F)c(F)c(C#N)c(F)c1F</smiles>

General Procedure A was followed, except with $\mathrm{D}_{2} \mathrm{O}$ instead of $\mathrm{H}_{2} \mathrm{O}$. Colorless crystalline solid. Spectra agreed with nondeuterated spectra, except as shown. HRMS (ESI/ion trap) m/z: [M - $\left.\mathrm{H}^{+}\right]^{-}$Calcd for $\mathrm{C}_{16} \mathrm{H}_{9} \mathrm{DF}_{4} \mathrm{NO}_{2}$ 325.0716; Found 325.0730. 


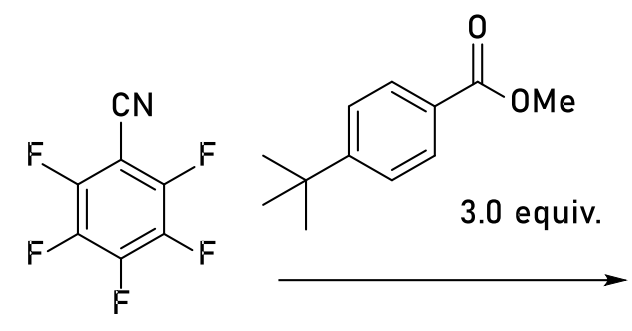

$4.0 \mathrm{mmol}$

DIPEA 1.1 equiv.

$\operatorname{Ir}$ (d'́bbpy)(d'bppy) ${ }_{2} \mathrm{PF}_{6} 0.125 \mathrm{~mol} \%$

Acetonitrile $0.2 \mathrm{M}$

$D_{2} 015$ equiv.

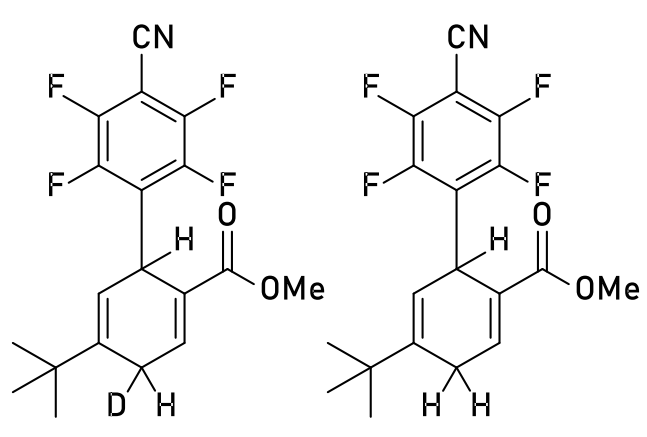

(23)

General Procedure A was followed, except with $\mathrm{D}_{2} \mathrm{O}$ instead of $\mathrm{H}_{2} \mathrm{O}$. Colorless crystalline solid Spectra agreed with nondeuterated spectra, except as shown. HRMS (ESI/ion trap) m/z: [M - $\left.\mathrm{H}^{+}\right]^{-}$Calcd for $\mathrm{C}_{19} \mathrm{H}_{15} \mathrm{DF}_{4} \mathrm{NO}_{2}{ }^{-}$ 367.1185; Found 367.1195. 


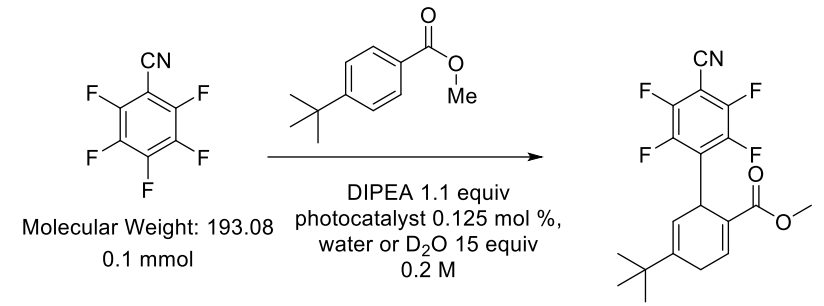

\begin{tabular}{|c|c|c|c|c|c|c|}
\hline \multicolumn{7}{|c|}{ Diene product formation (\%) } \\
\hline time (min) & & 0 & 2.5 & 7.5 & 12.5 & 17.5 \\
\hline \multirow{3}{*}{$\begin{array}{l}\text { O } \\
\text { I }\end{array}$} & JD5121A & 0 & 0.17 & 2.08 & 3.78 & 4.9 \\
\hline & JD5121B & 0 & 0.24 & 2.14 & 3.82 & 4.79 \\
\hline & JD5121C & 0 & 0.28 & 2.26 & 3.92 & 4.86 \\
\hline \multirow{3}{*}{$\stackrel{\text { }}{\text { O }}$} & JD5121D & 0 & 0.25 & 1.48 & 2.68 & 3.37 \\
\hline & JD5121E & 0 & 0.27 & 1.63 & 2.81 & 3.5 \\
\hline & JD5121F & 0 & 0.26 & 1.54 & 2.76 & 3.48 \\
\hline \multicolumn{7}{|l|}{ avg \% } \\
\hline $\begin{array}{l}\text { proteo } \\
\text { stddev }\end{array}$ & & 0 & 1.15 & 10.80 & 19.20 & 24.25 \\
\hline proteo & & 0 & 0.05 & 0.07 & 0.06 & 0.05 \\
\hline \multicolumn{7}{|l|}{ avg \% } \\
\hline deutero & & 0 & 1.30 & 7.75 & 13.75 & 17.25 \\
\hline \multicolumn{7}{|l|}{ stddev } \\
\hline \multirow[t]{4}{*}{ deutero } & \multirow{2}{*}{\multicolumn{2}{|c|}{ slope }} & 0.01 & 0.06 & 0.05 & 0.06 \\
\hline & & & \multicolumn{3}{|l|}{ intercept } & \multirow[b]{2}{*}{$\mathrm{KH} / \mathrm{KD}$} \\
\hline & $\mathrm{KH}$ & 1.48804878 & \multicolumn{2}{|l|}{0.00} & & \\
\hline & $\mathrm{KD}$ & 1.04743902 & 0.00 & & & 1.42065433 \\
\hline \multicolumn{7}{|c|}{ HDF product formation (\%) } \\
\hline \multirow[t]{7}{*}{ time $(\min )$} & & 0 & 2.5 & 7.5 & 12.5 & 17.5 \\
\hline & JD5121A & 0 & 0.4 & 2.97 & 4.76 & 5.8 \\
\hline & JD5121B & 0 & 0.49 & 2.91 & 4.65 & 5.58 \\
\hline & JD5121C & 0 & 0.55 & 3.04 & 4.78 & 5.61 \\
\hline & JD5121D & 0 & 0.72 & 2.41 & 3.46 & 4.04 \\
\hline & JD5121E & 0 & 0.76 & 2.54 & 3.59 & 3.59 \\
\hline & JD5121F & 0 & 0.71 & 2.42 & 3.53 & 4.08 \\
\hline \multicolumn{7}{|l|}{ avg \% } \\
\hline proteo & & 0 & 2.40 & 14.87 & 23.65 & 28.32 \\
\hline \multicolumn{7}{|l|}{ stddev } \\
\hline proteo & & 0 & 0.06 & 0.05 & 0.06 & 0.10 \\
\hline \multicolumn{7}{|l|}{$\operatorname{avg} \%$} \\
\hline deutero & & 0 & 3.65 & 12.28 & 17.63 & 19.52 \\
\hline \multicolumn{7}{|l|}{ stddev } \\
\hline \multirow[t]{4}{*}{ deutero } & \multirow{2}{*}{\multicolumn{2}{|c|}{ slope }} & 0.02 & 0.06 & 0.05 & 0.22 \\
\hline & & & intercept & & & \multirow[b]{2}{*}{$\mathrm{KH} / \mathrm{KD}$} \\
\hline & $\mathrm{KH}$ & 1.73073171 & 0.00 & & & \\
\hline & $\mathrm{KD}$ & 1.16361789 & 0.00 & & & 1.48737118 \\
\hline & & Conve & sion (\%) & & & \\
\hline time (min) & & 0 & 2.5 & 7.5 & 12.5 & 17.5 \\
\hline & JD5121A & 0 & 15.26 & 8.82 & 3.4 & 0.64 \\
\hline & JD5121B & 0 & 14.4 & 7.8 & 2.77 & 0.05 \\
\hline & JD5121C & 0 & 14.03 & 7.24 & 2.51 & 0.04 \\
\hline & JD5121D & 0 & 14.23 & 7.89 & 3.56 & 1.21 \\
\hline & JD5121E & 0 & 13.96 & 7.6 & 3.22 & 0.92 \\
\hline & JD5121F & 0 & 13.89 & 8 & 3.33 & 0.94 \\
\hline $\operatorname{avg} \%$ & & & & & & \\
\hline $\begin{array}{l}\text { proteo } \\
\text { stddev }\end{array}$ & & 0 & 27.18 & 60.23 & 85.53 & 98.78 \\
\hline proteo & & 0 & 0.52 & 0.65 & 0.37 & 0.28 \\
\hline avg \% & & & & & & \\
\hline deutero & & 0 & 29.87 & 60.85 & 83.15 & 94.88 \\
\hline
\end{tabular}
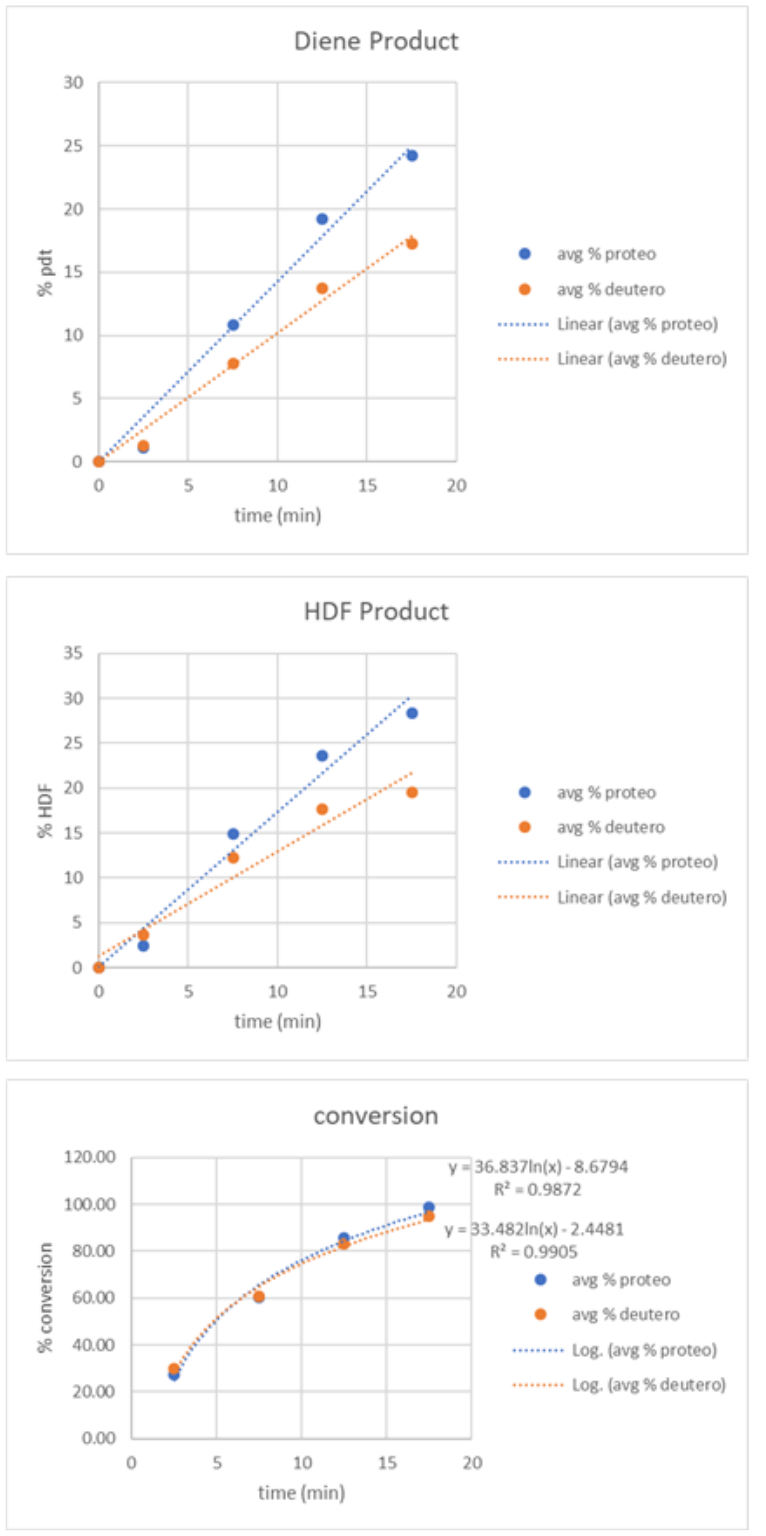
stddev

deutero

0.15

0.17

0.14

$K H$

slope

intercept

KH $\quad 5.57910569$

0.00

0.00

$\mathrm{KH} / \mathrm{KD}$

$\mathrm{KD}$

5.27256098

1.05813962<smiles>Fc1c(F)c(F)c(F)c(F)c1F</smiles>

Molecular Weight: 246.9620 photocatalyst $0.125 \mathrm{~mol} \%$,
water or $\mathrm{D}_{2} \mathrm{O} 15$ equiv $0.1 \mathrm{mmol}$

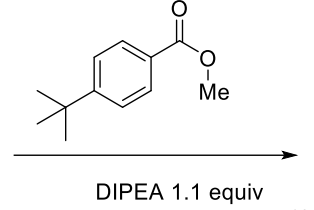
$0.2 \mathrm{M}$

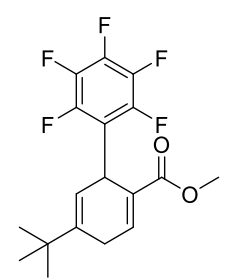

Formation of diene

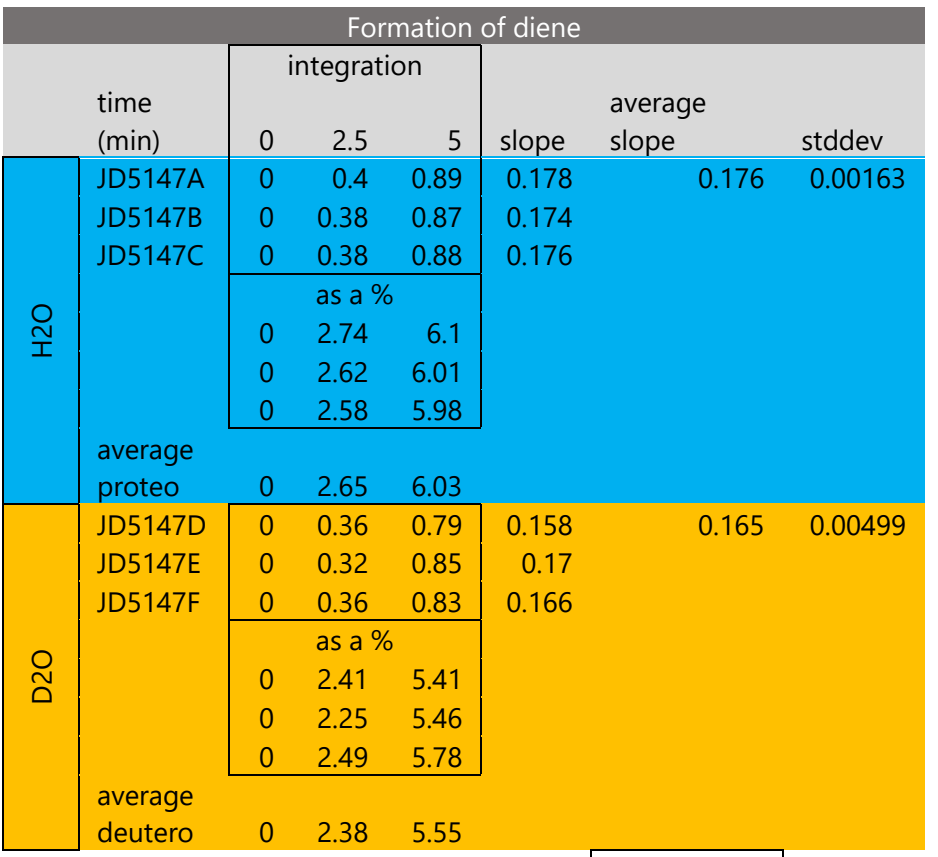

1.069

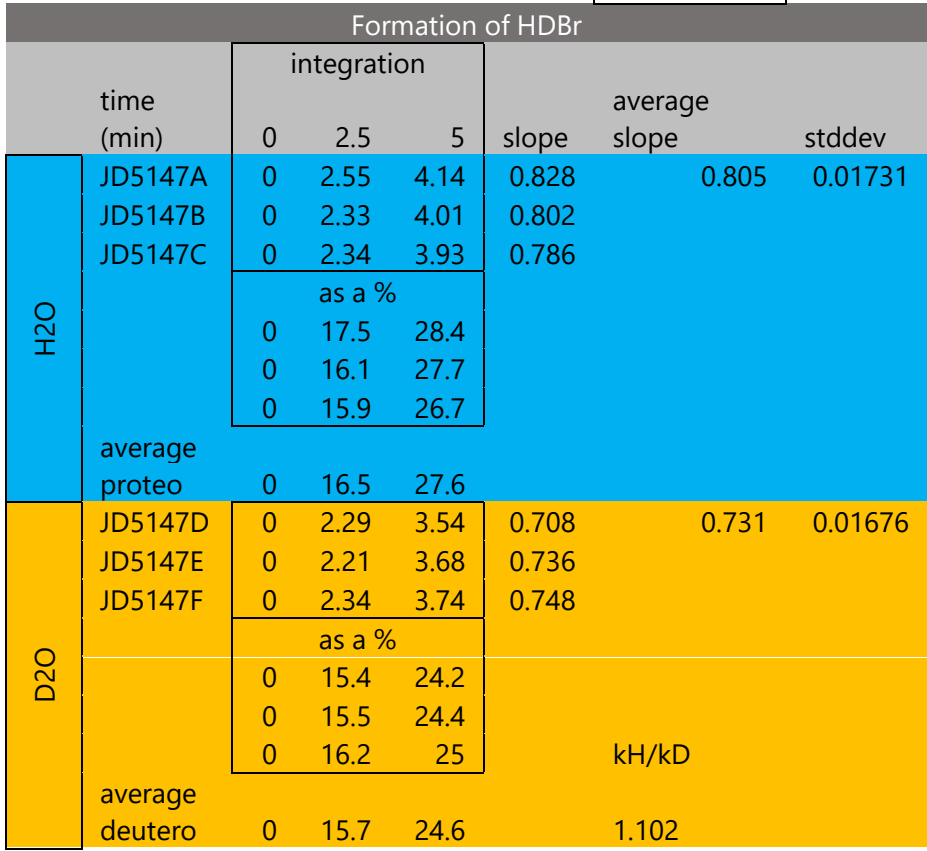

Average Rate of Formation of the 1,4 diene from bromopentafluorobenzene and methyl 4-tBu-benzoate
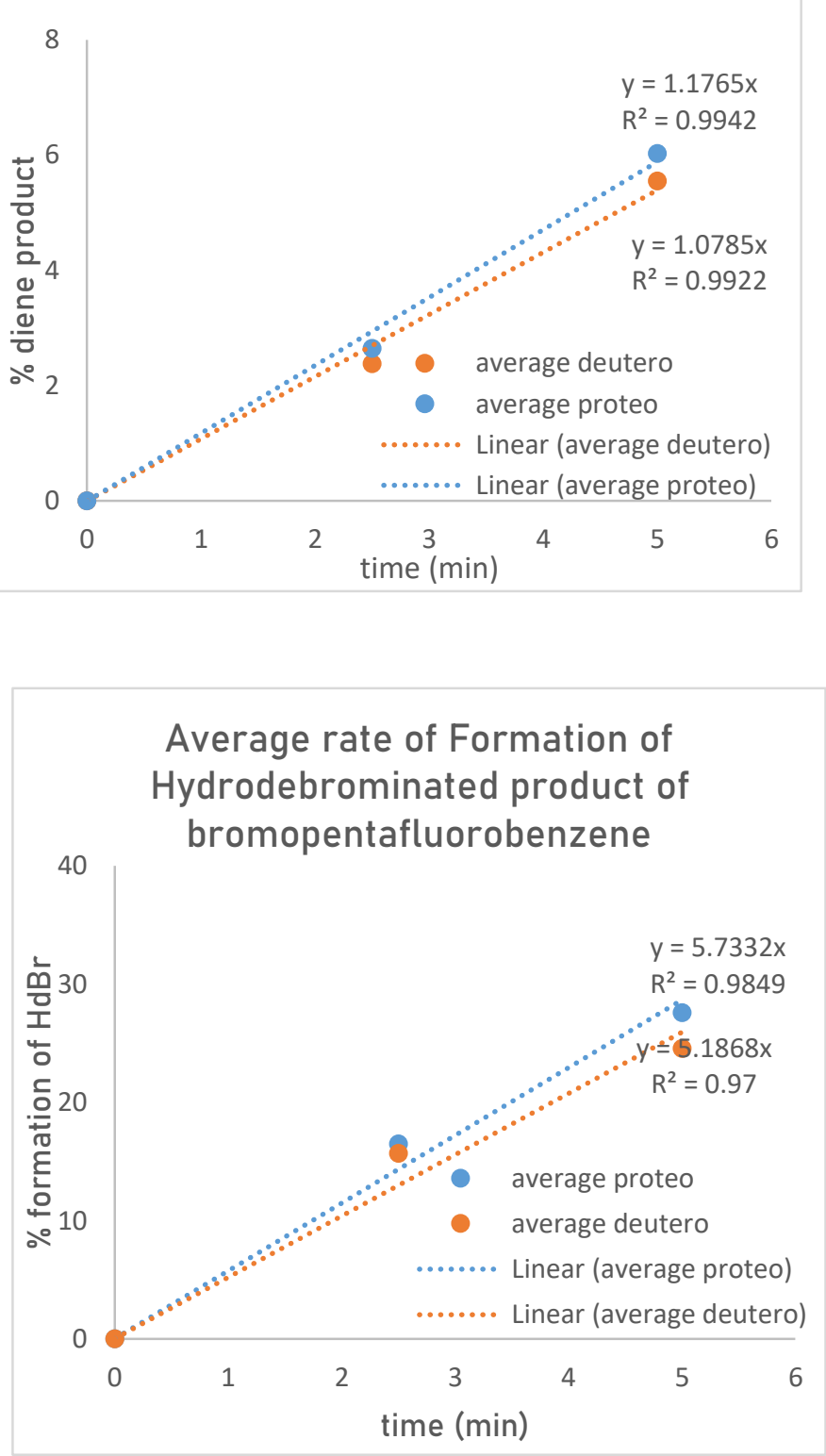
Conversion of SM

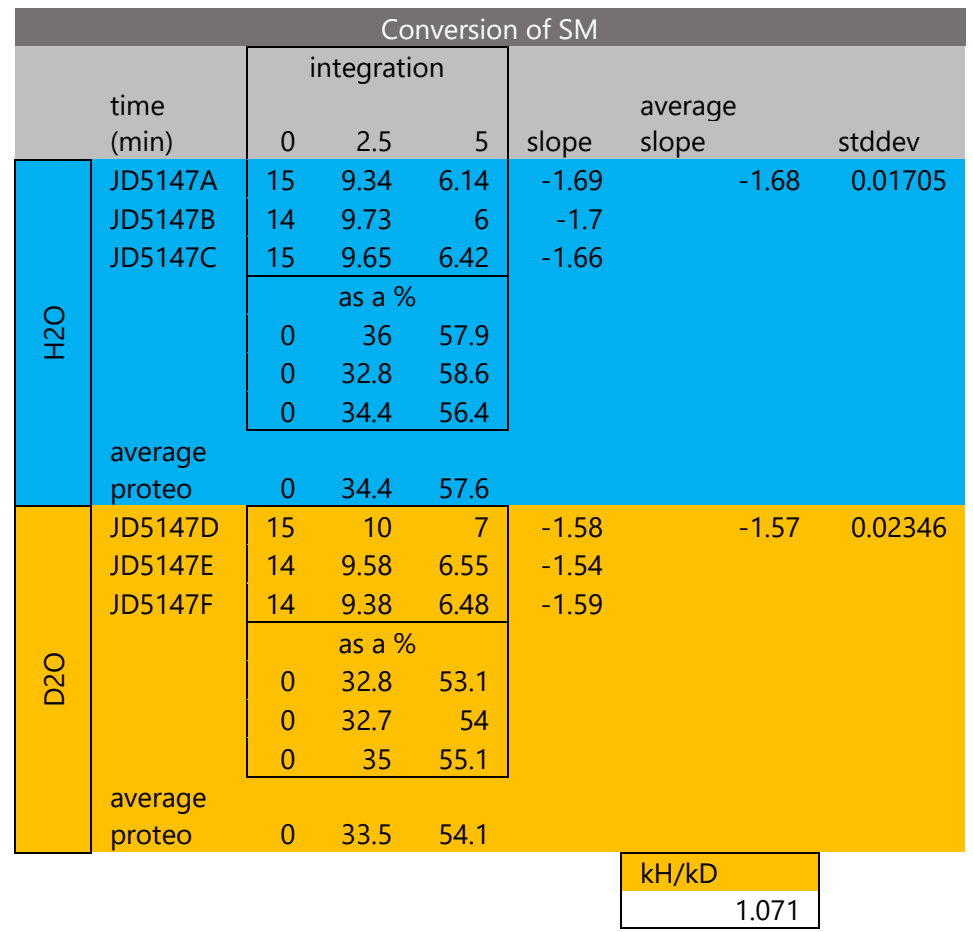

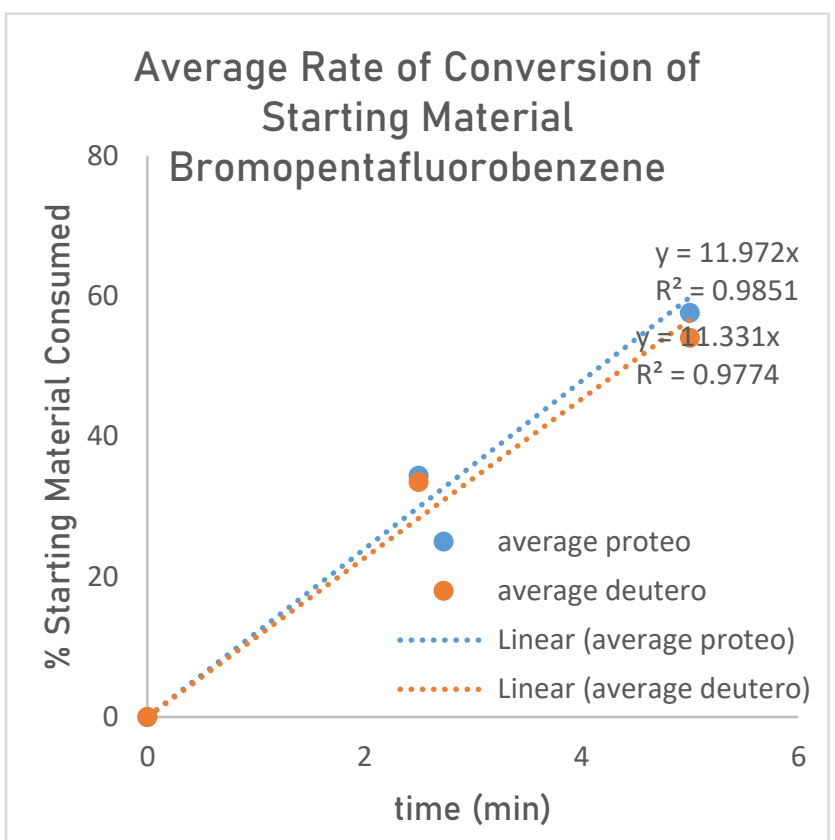




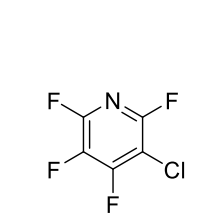

Molecular Weight: 185.51 photocatalyst $0.125 \mathrm{~mol} \%$, $0.1 \mathrm{mmol}$

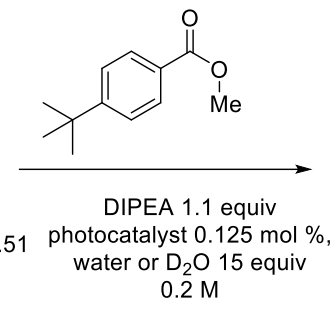

Formation of diene

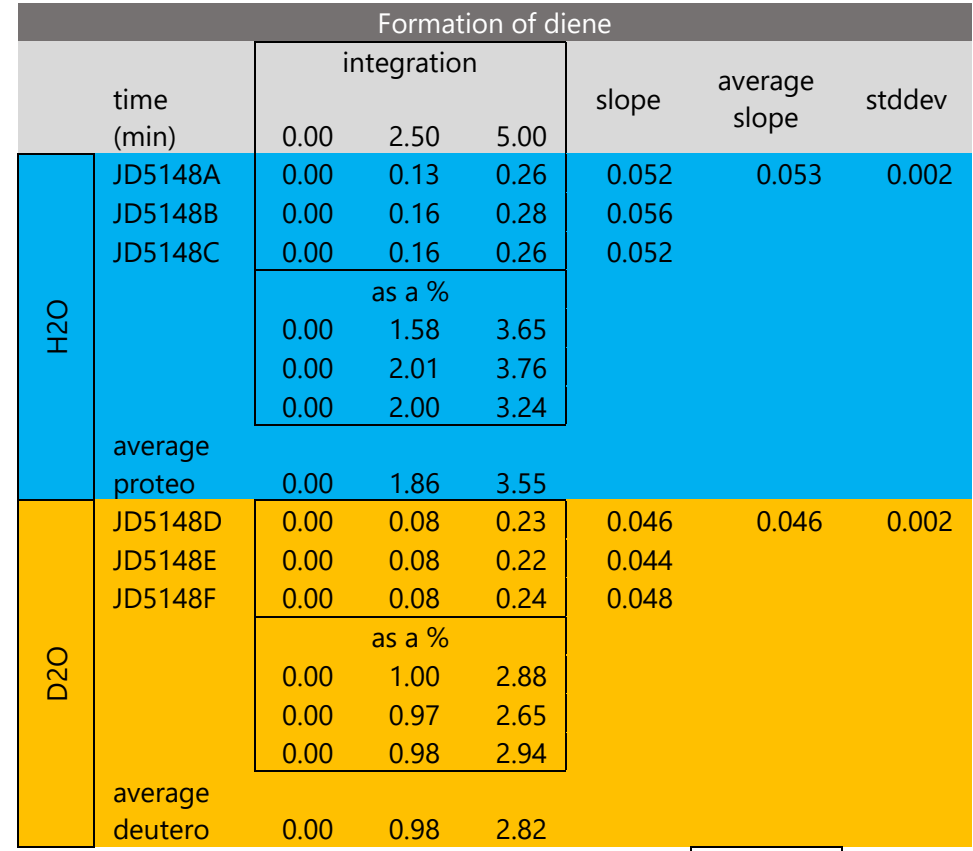

$\mathrm{kH} / \mathrm{kD}$

1.1363

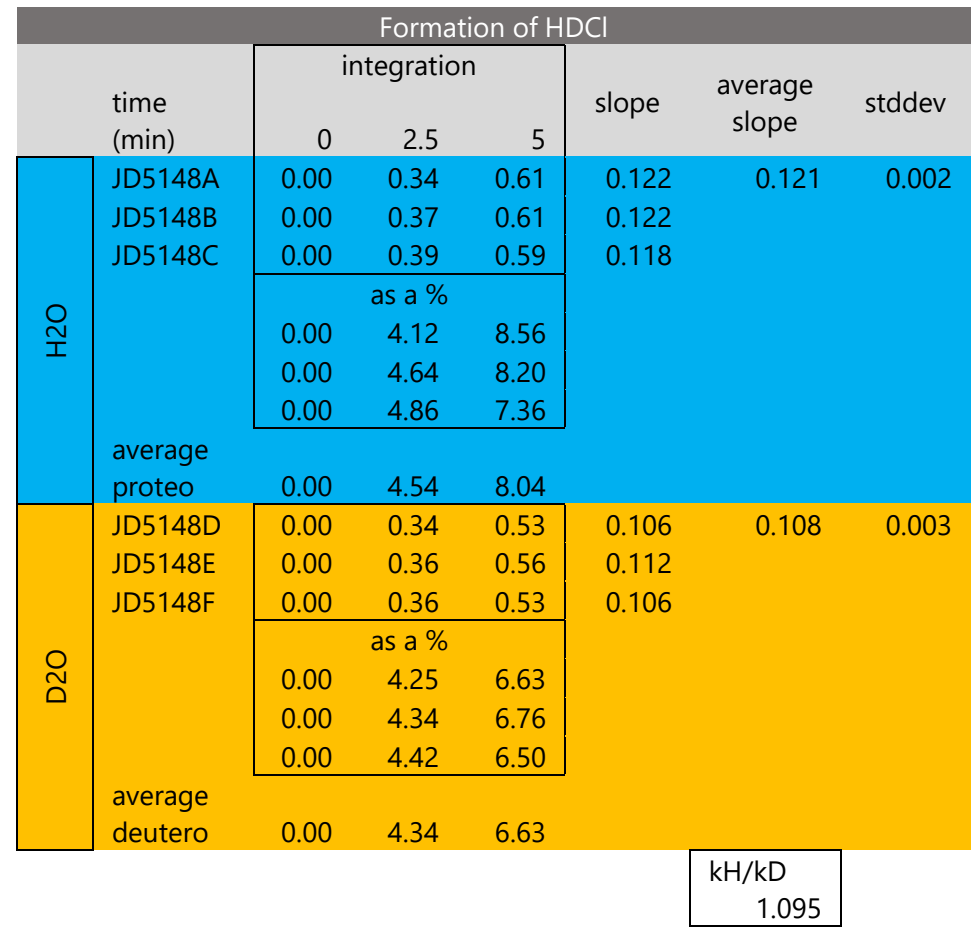

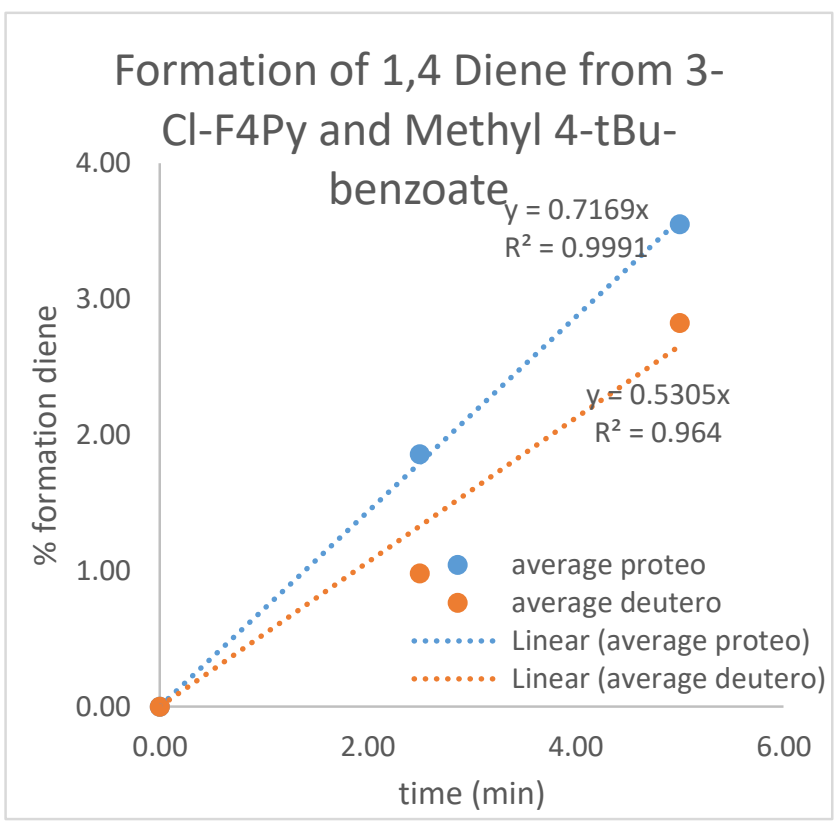

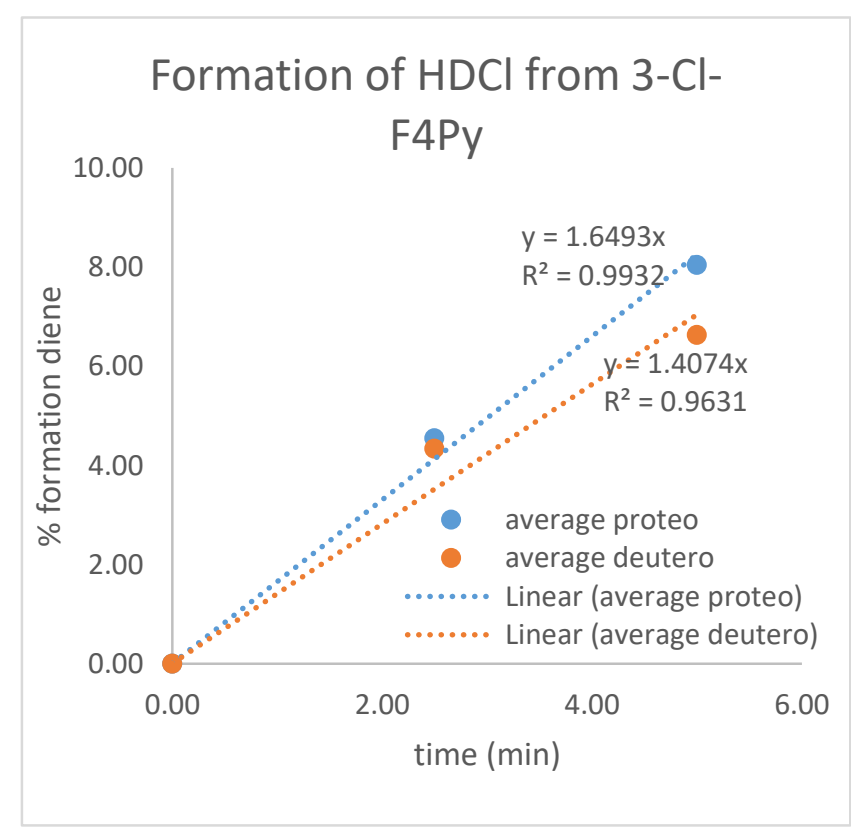




\begin{tabular}{|c|c|c|c|c|c|c|c|}
\hline \multicolumn{8}{|c|}{ Consumption of SM } \\
\hline & \multirow{2}{*}{$\begin{array}{l}\text { time } \\
\text { (min) }\end{array}$} & \multicolumn{3}{|c|}{ integration } & \multirow{2}{*}{ slope } & \multirow{2}{*}{$\begin{array}{l}\text { average } \\
\text { slope }\end{array}$} & \multirow{2}{*}{ stddev } \\
\hline & & 0 & 2.5 & 5 & & & \\
\hline \multirow{8}{*}{$\stackrel{O}{I}$} & JD5148A & 8.25 & 7.13 & 6.90 & \multirow{8}{*}{$\begin{array}{l}-0.270 \\
-0.180 \\
-0.252\end{array}$} & \multirow[t]{8}{*}{-0.234} & \multirow[t]{8}{*}{0.039} \\
\hline & JD5148B & 7.97 & 7.44 & 7.07 & & & \\
\hline & \multirow[t]{5}{*}{ JD5148C } & 8.02 & 7.36 & 6.76 & & & \\
\hline & & \multicolumn{3}{|c|}{ as a $\%$} & & & \\
\hline & & 0.00 & 13.58 & 16.36 & & & \\
\hline & & 0.00 & 6.65 & 11.29 & & & \\
\hline & & 0.00 & 8.23 & 15.71 & & & \\
\hline & \multicolumn{4}{|l|}{ average } & & & \\
\hline \multirow{8}{*}{$\stackrel{\text { ֻ }}{\Delta}$} & JD5148D & 8.00 & 7.33 & 6.95 & \multirow{8}{*}{$\begin{array}{l}-0.210 \\
-0.278 \\
-0.200\end{array}$} & \multirow[t]{8}{*}{-0.229} & \multirow[t]{8}{*}{0.035} \\
\hline & JD5148E & 8.29 & 7.35 & 6.90 & & & \\
\hline & \multirow[t]{5}{*}{ JD5148F } & 8.15 & 7.30 & 7.15 & & & \\
\hline & & \multicolumn{3}{|c|}{ as a $\%$} & & & \\
\hline & & 0.00 & 8.38 & 13.13 & & & \\
\hline & & 0.00 & 11.34 & 16.77 & & & \\
\hline & & 0.00 & 10.43 & 12.27 & & & \\
\hline & $\begin{array}{l}\text { average } \\
\text { deutero }\end{array}$ & 0.00 & 10.05 & 14.05 & & & \\
\hline & & & & & & $\begin{array}{c}\mathrm{kH} / \mathrm{kD} \\
1.0203\end{array}$ & \\
\hline
\end{tabular}

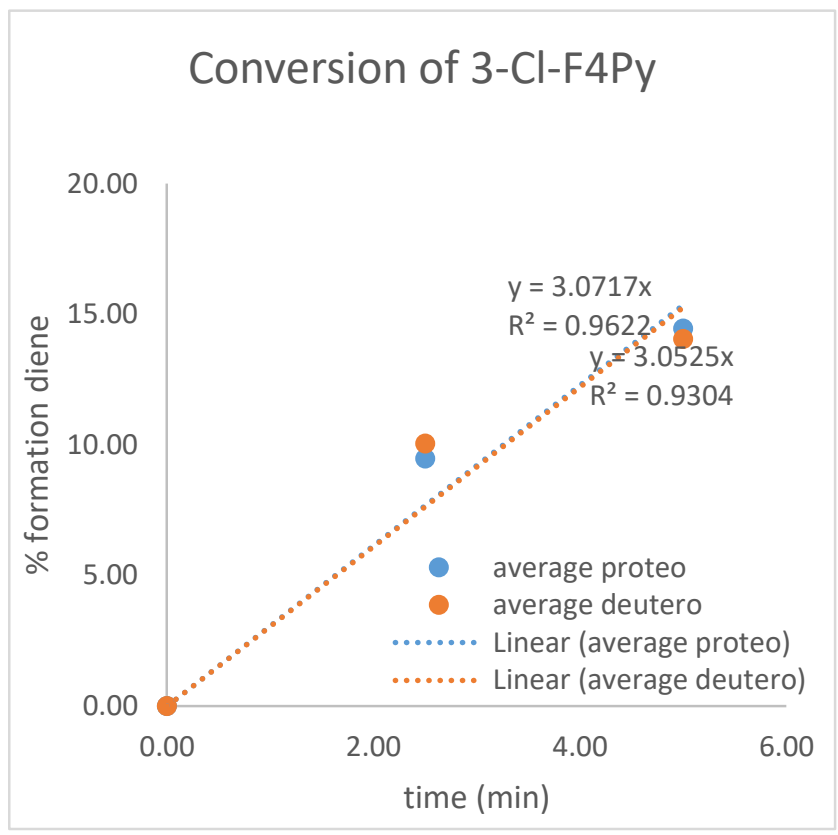




\section{NMR Spectra}

(5) methyl 5-(tert-butyl)-4'-cyano-2', 3', 5',6'-tetrafluoro-1,4-dihydro-[1,1'-biphenyl]-2-carboxylate

${ }^{19} \mathrm{~F}$ NMR $\left(\mathrm{CD}_{3} \mathrm{CN}, 376 \mathrm{MHz}\right)$

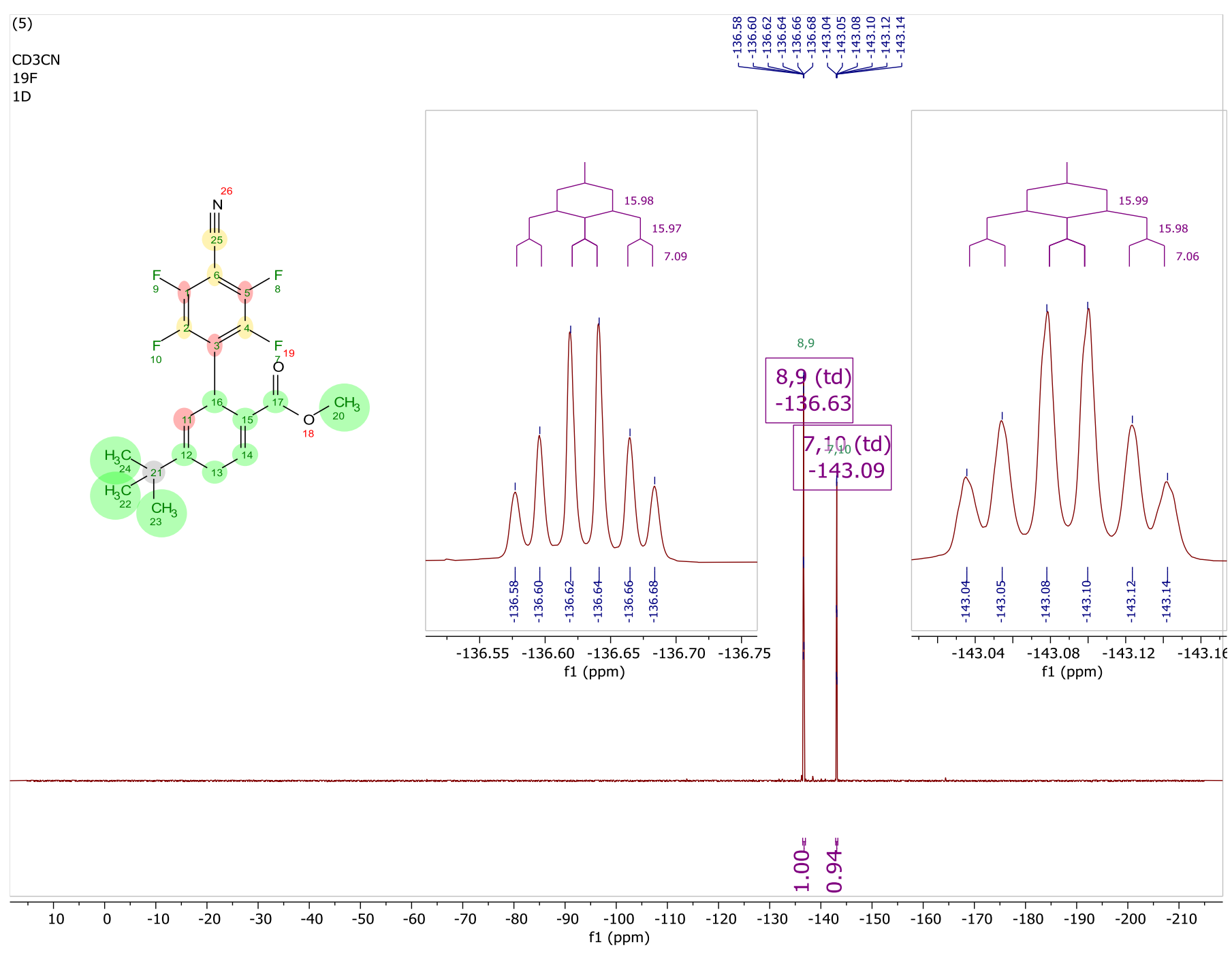


${ }^{1} \mathrm{H}$ NMR $\left(\mathrm{CD}_{3} \mathrm{CN}, 400 \mathrm{MHz}\right)$

(5)

$\mathrm{CD} 3 \mathrm{CN}$

$1 \mathrm{H}$

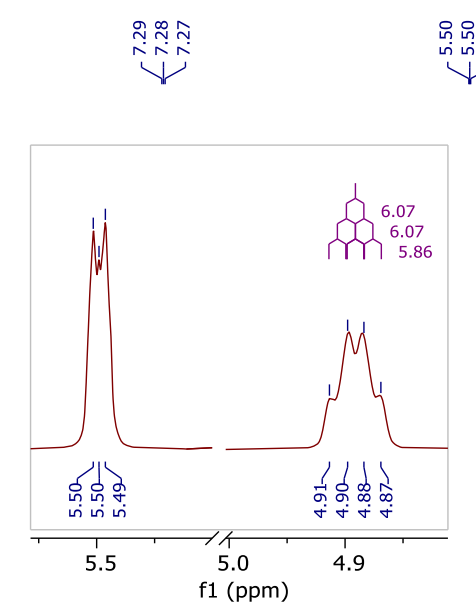

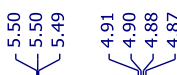

资
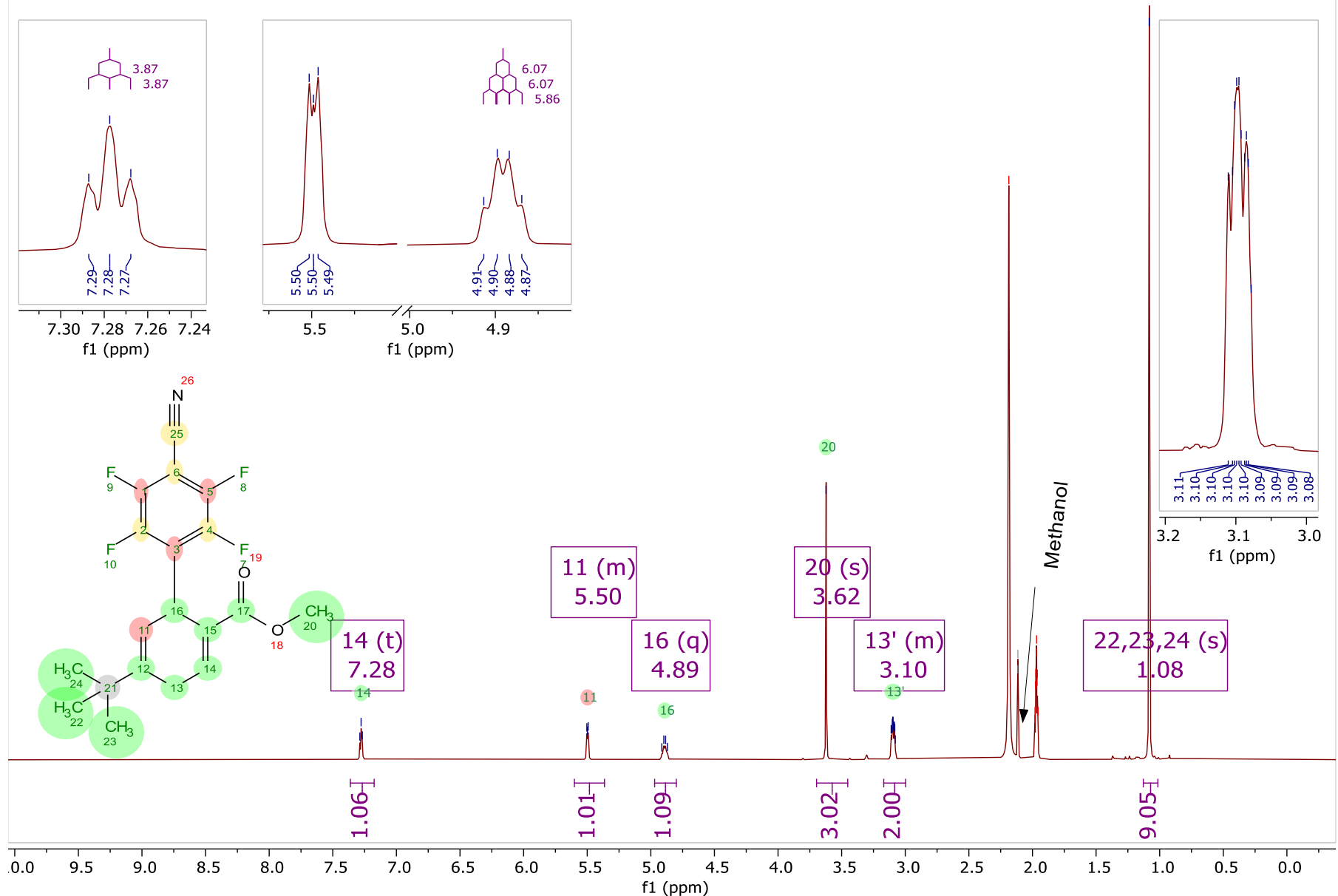
${ }^{13} \mathbf{C}\left\{{ }^{1} \mathrm{H}\right\}$ NMR $\left(\mathrm{CD}_{3} \mathrm{CN}, 151 \mathrm{MHz}\right)$

(5)

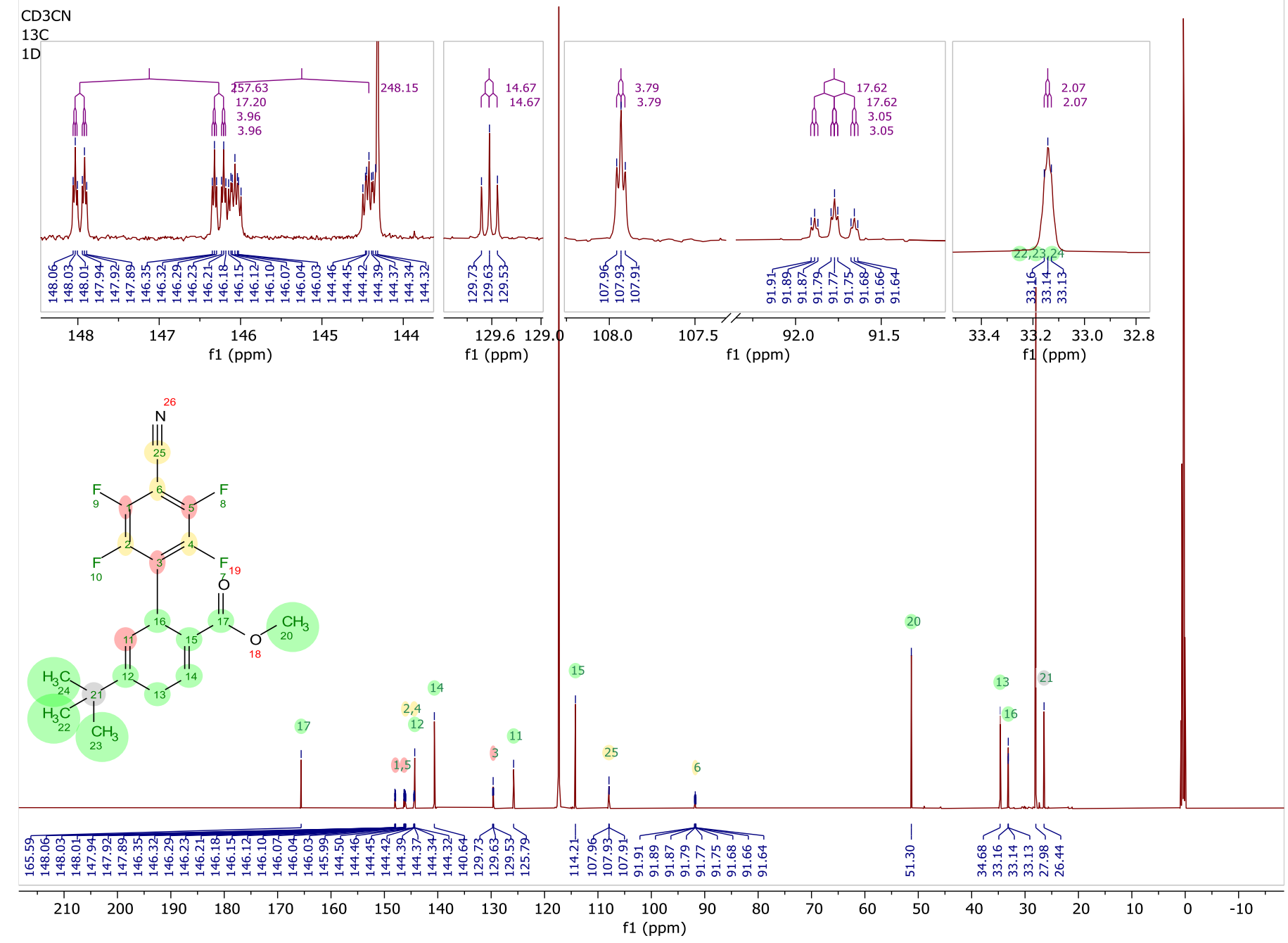


${ }^{1} \mathrm{H}-{ }^{1} \mathrm{H}$ NOESY

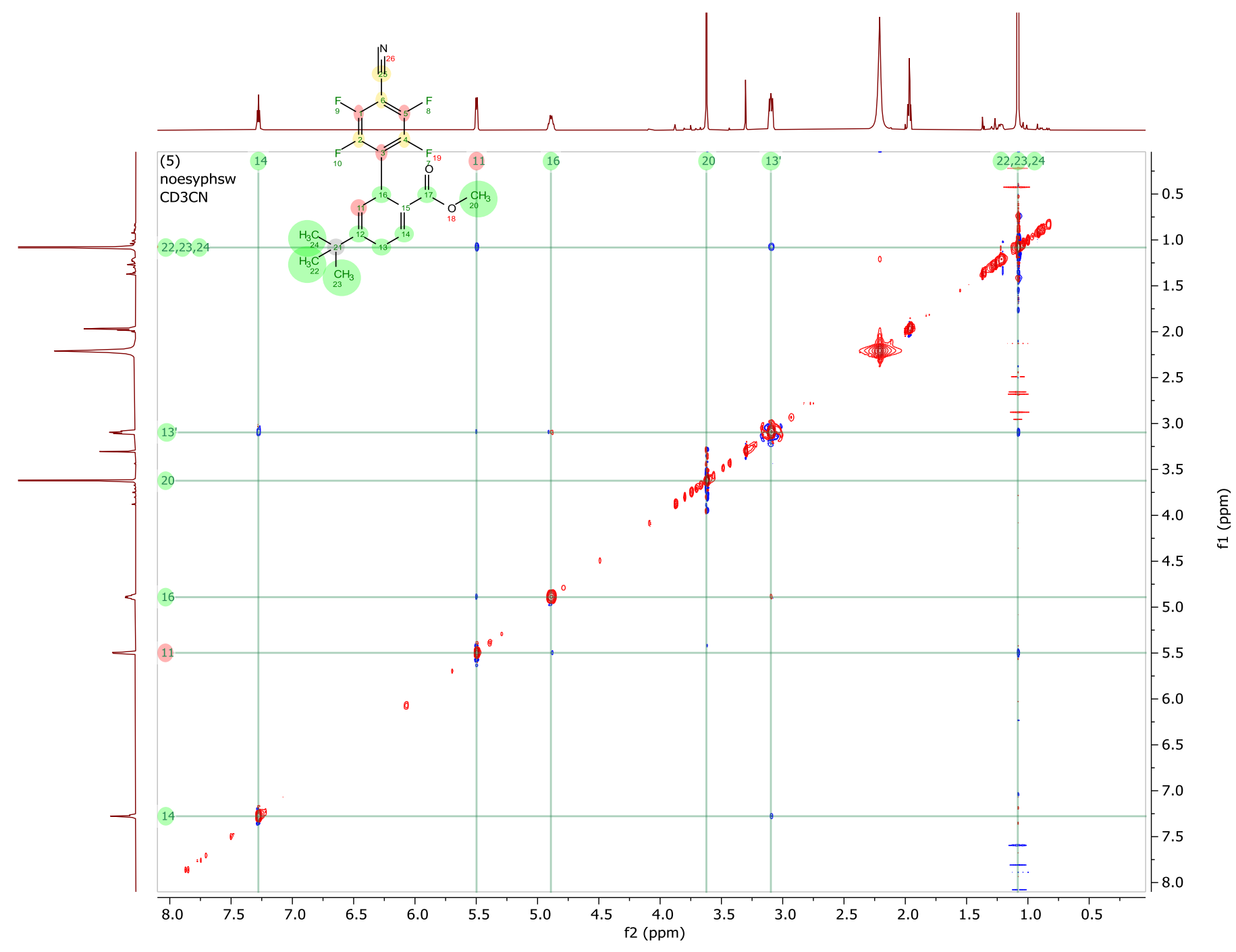


${ }^{1} \mathrm{H}-{ }^{1} \mathrm{H} \cos Y$

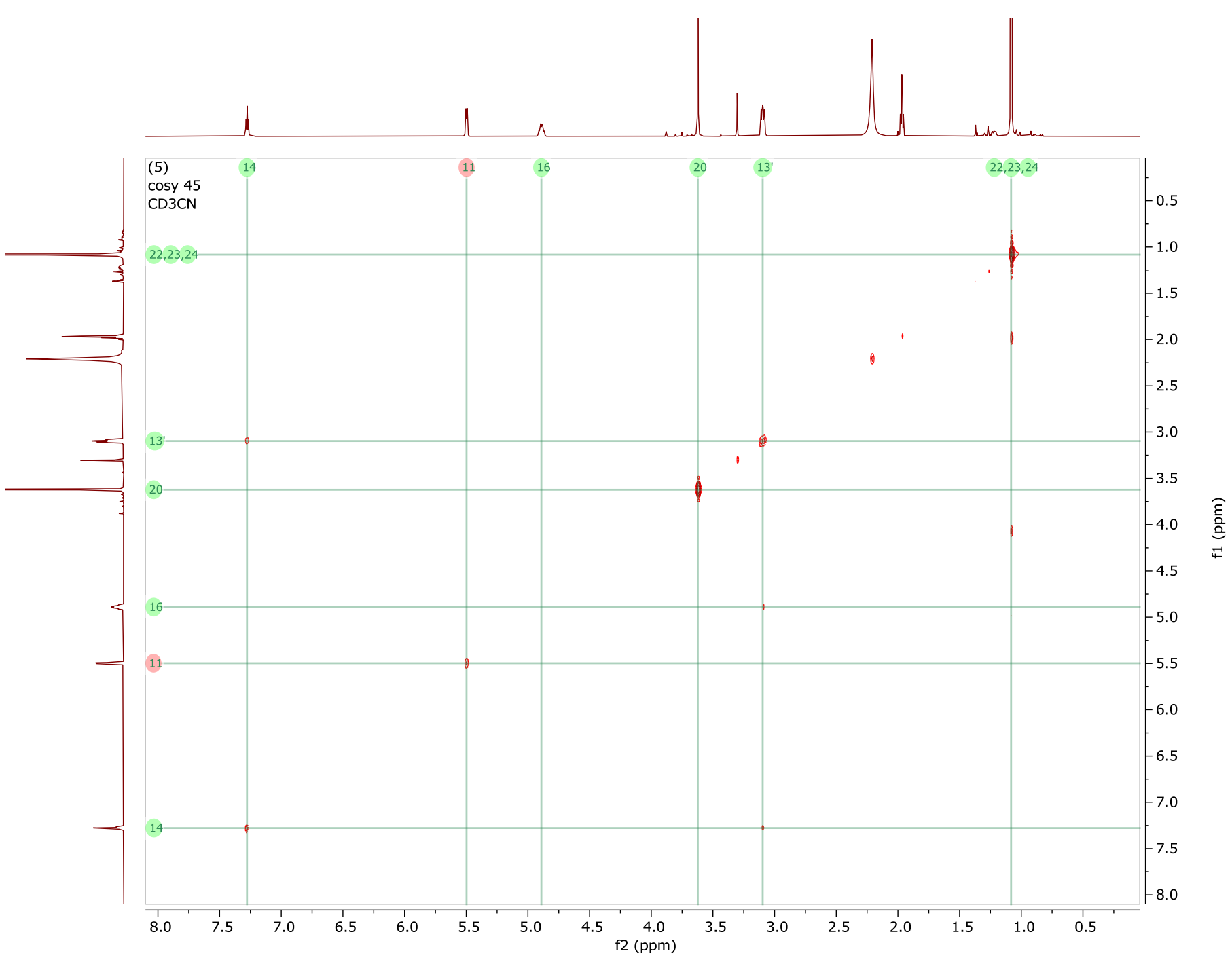


${ }^{1} \mathrm{H}-{ }^{19} \mathrm{~F}$ HOESY

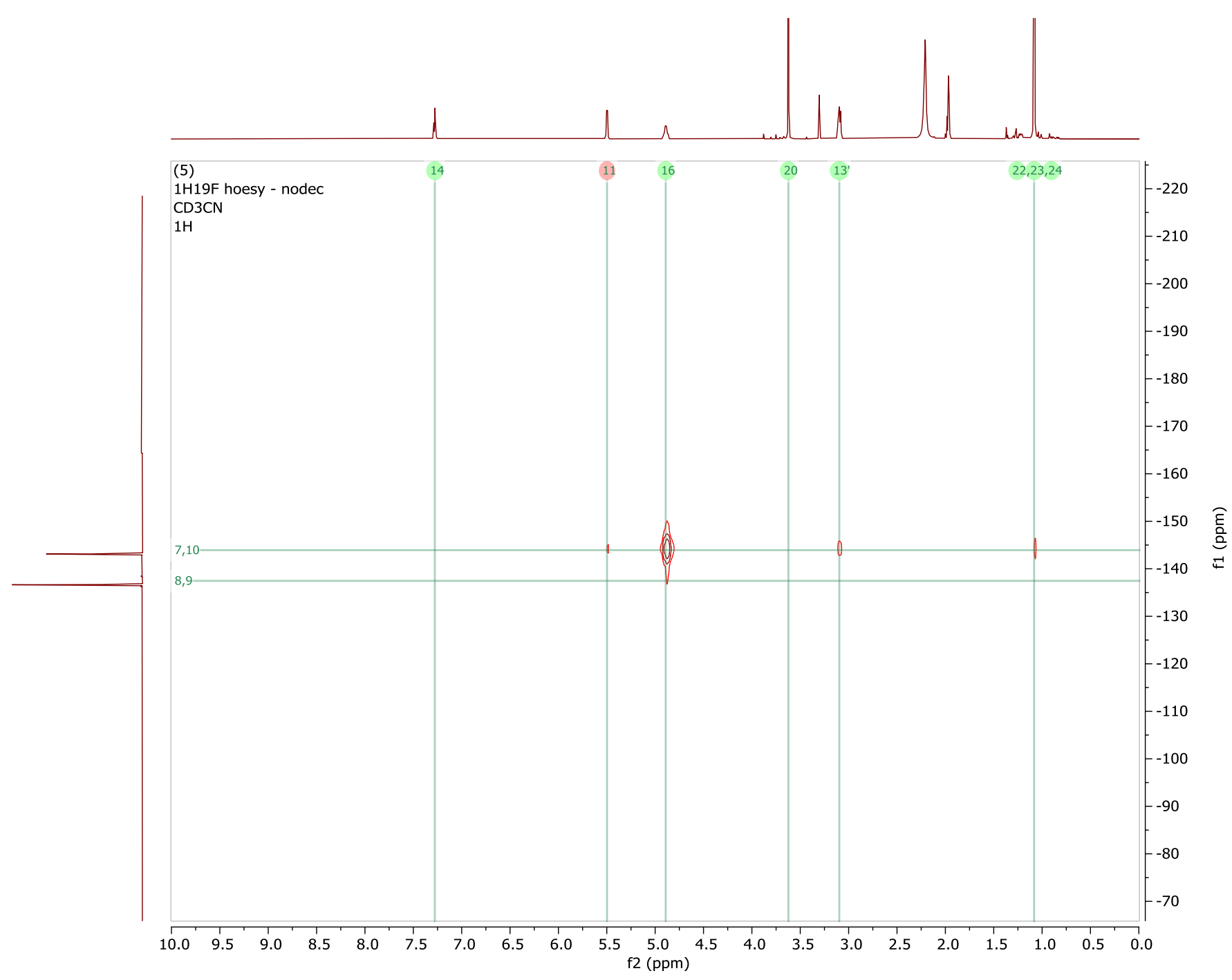


Attached Proton Test

attached proton test

CD3CN
$13 \mathrm{C}$
JMOD

JMOD

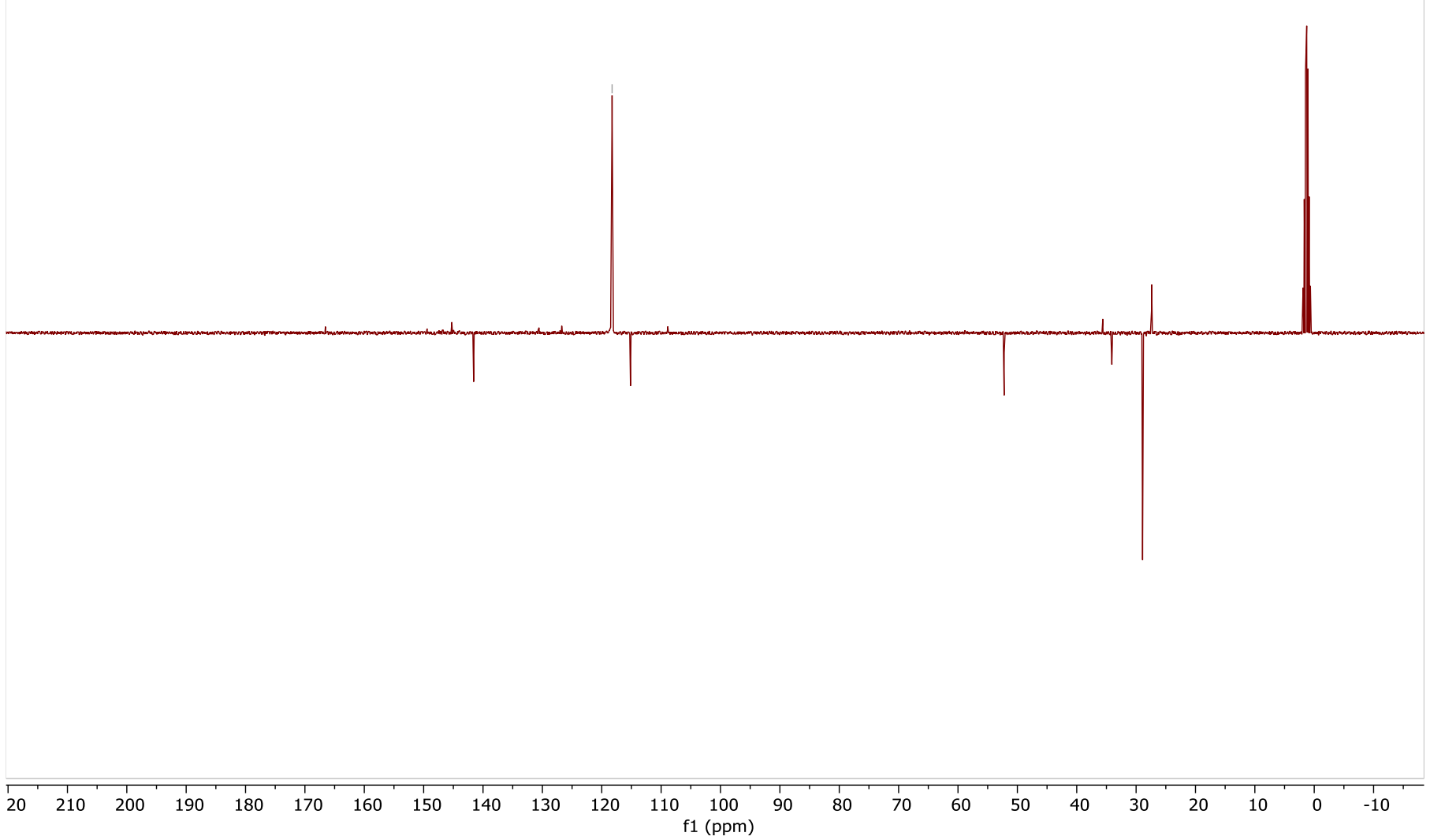




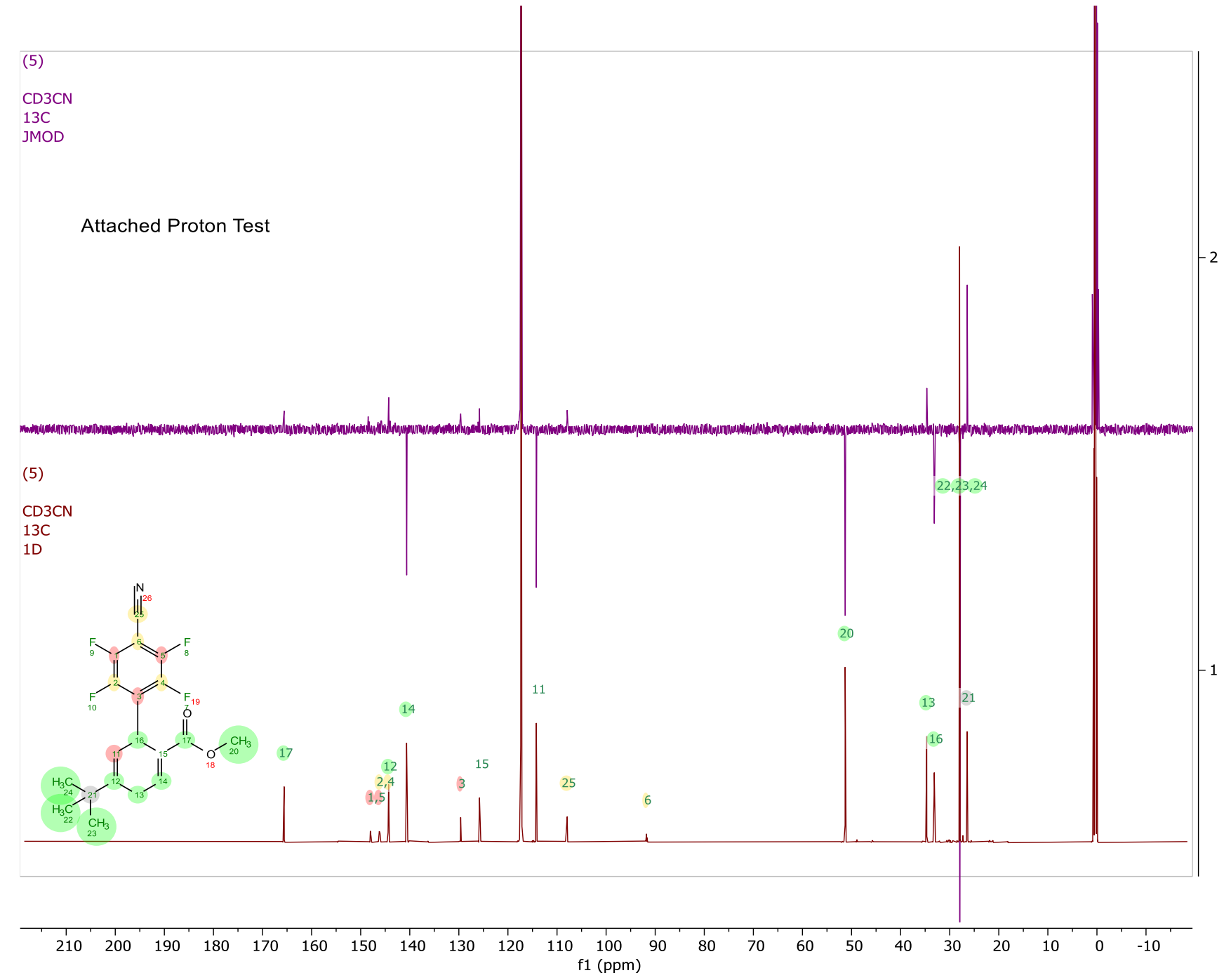


(6) methyl 4' -cyano-2',3',5',6' -tetrafluoro-5-methyl-1,4-dihydro-[1,1'-biphenyl]-2-carboxylate.

${ }^{19}$ F NMR $\left(\mathrm{CDCl}_{3}, 376 \mathrm{MHz}\right)$

(6)

$\mathrm{CDCl} 3$
$19 \mathrm{~F}$

$19 \mathrm{~F}$
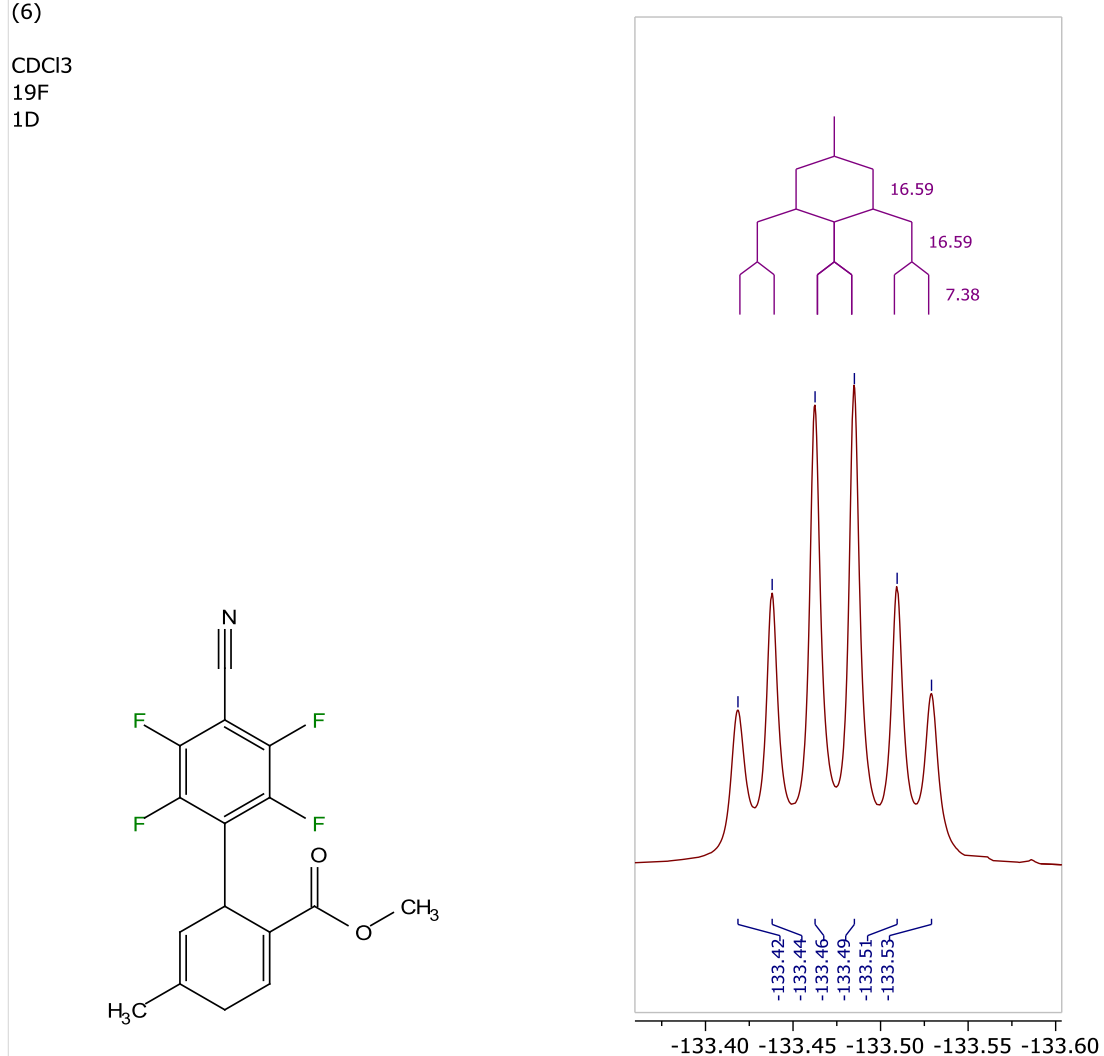

$-133.40-133.45-133.50-133.55-133.60$

8,11
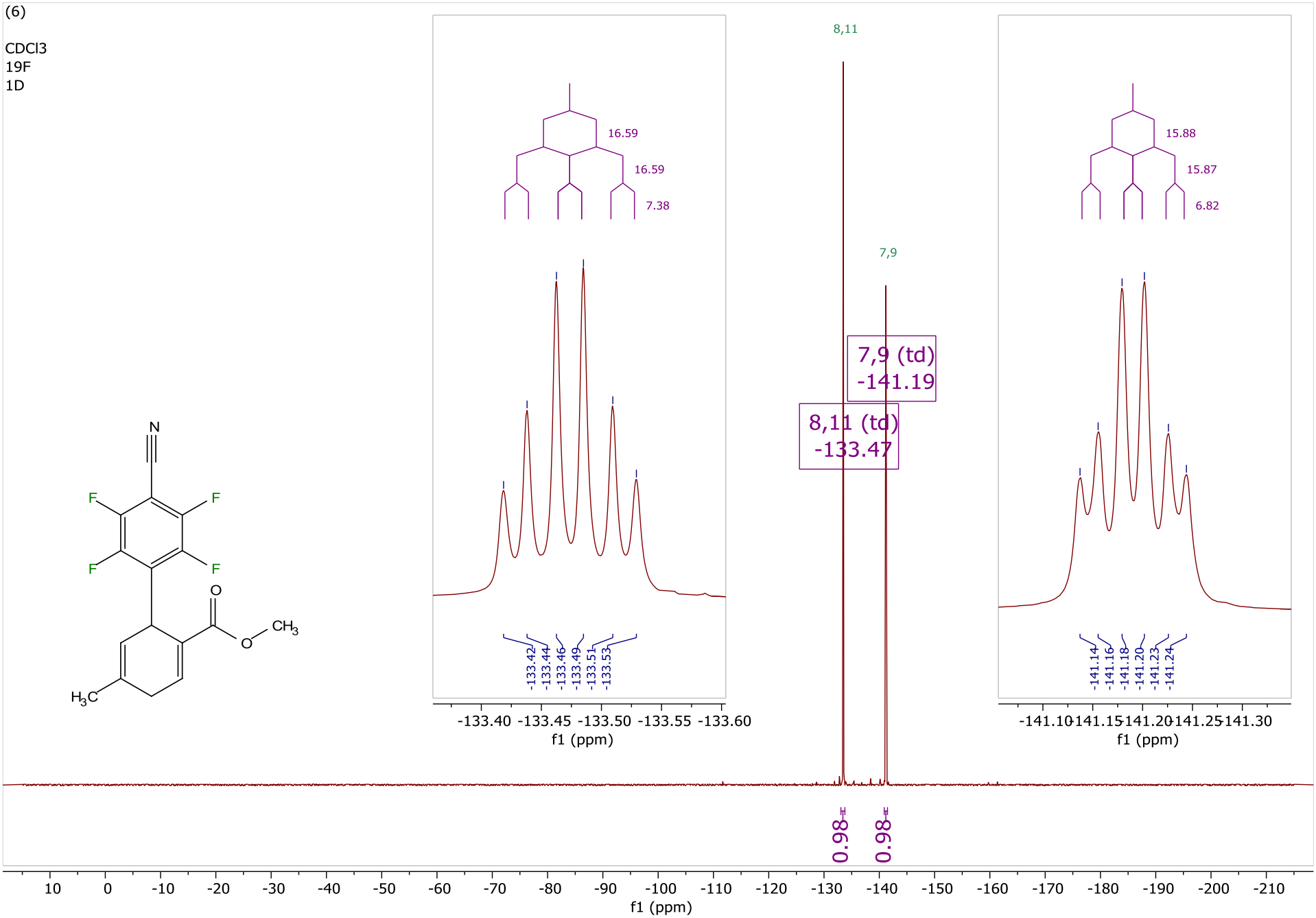
${ }^{\mathbf{1}} \mathbf{H} \mathbf{N M R}\left(\mathrm{CDCl}_{3}, 400 \mathrm{MHz}\right)$

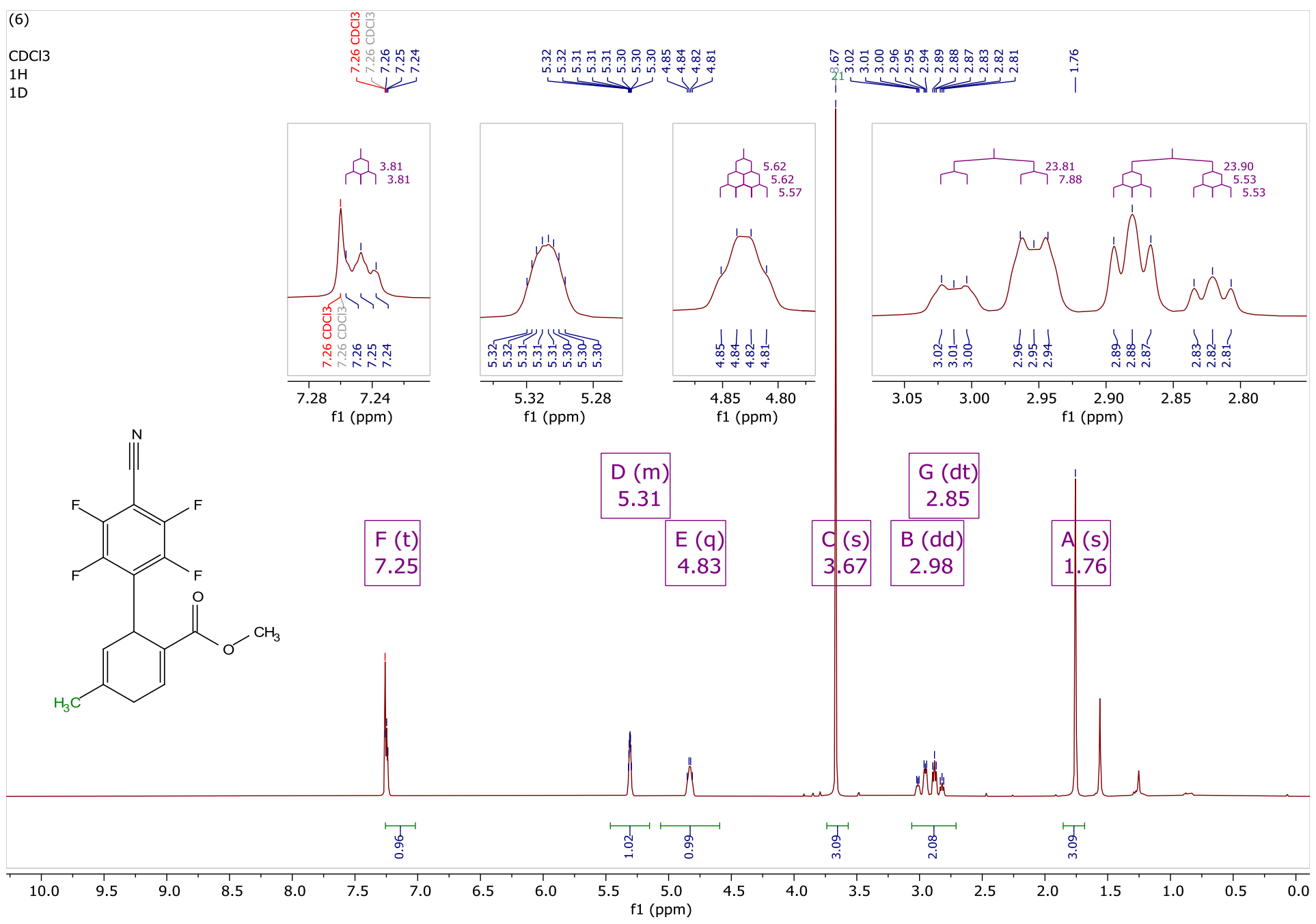




\section{${ }^{13} \mathrm{C}\left\{{ }^{1} \mathrm{H}\right\}$ NMR $\left(\mathrm{CDCl}_{3}, 101 \mathrm{MHz}\right)$}

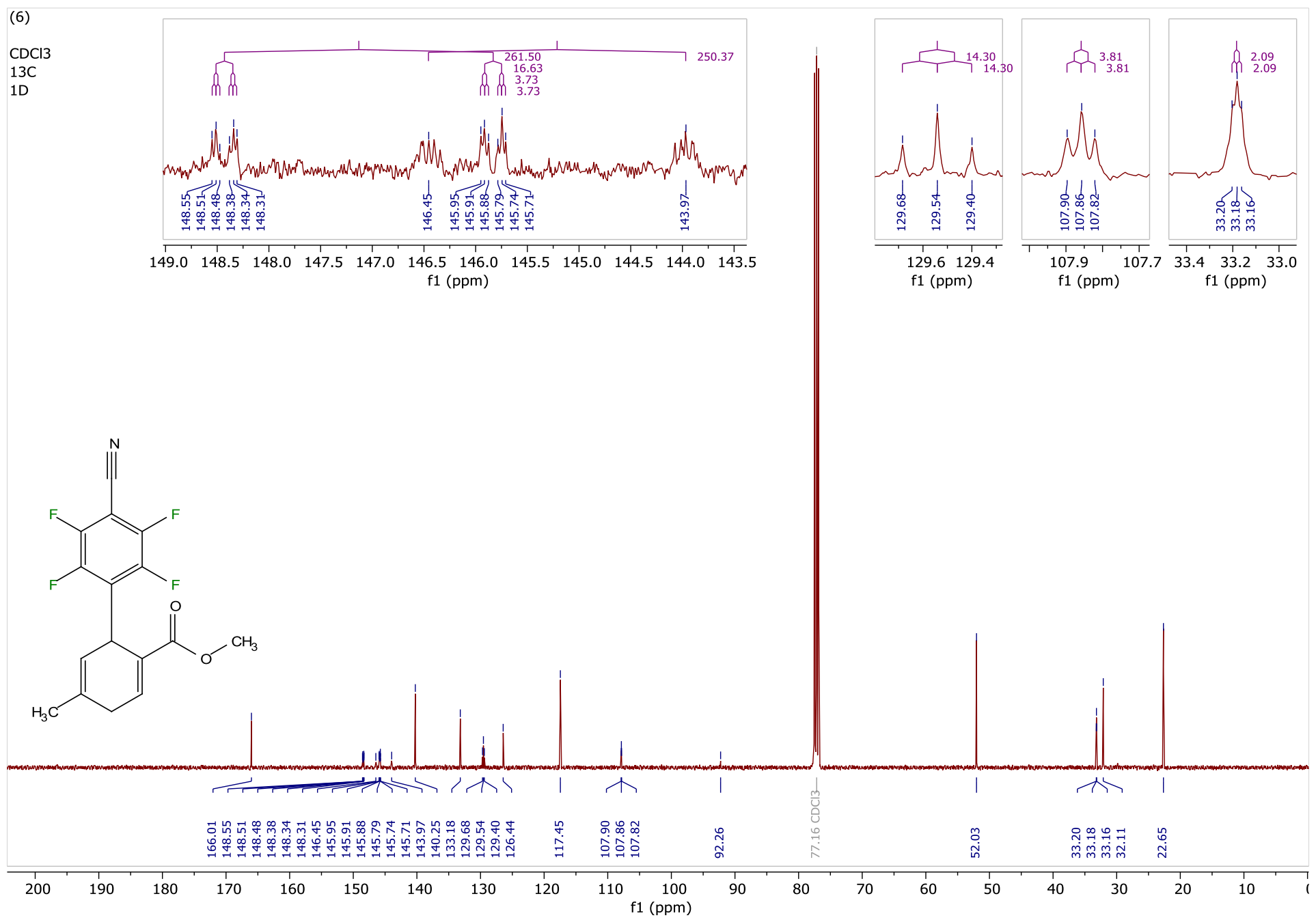


(7) 4' -cyano-2',3',5',6' -tetrafluoro-N,N-diisopropyl-1,4-dihydro-[1,1'-biphenyl]-2-carboxamide.

${ }^{19}$ F NMR $\left(\mathrm{CDCl}_{3}, 376 \mathrm{MHz}\right)$

(7)

$\mathrm{CDCl3}$

$19 F$
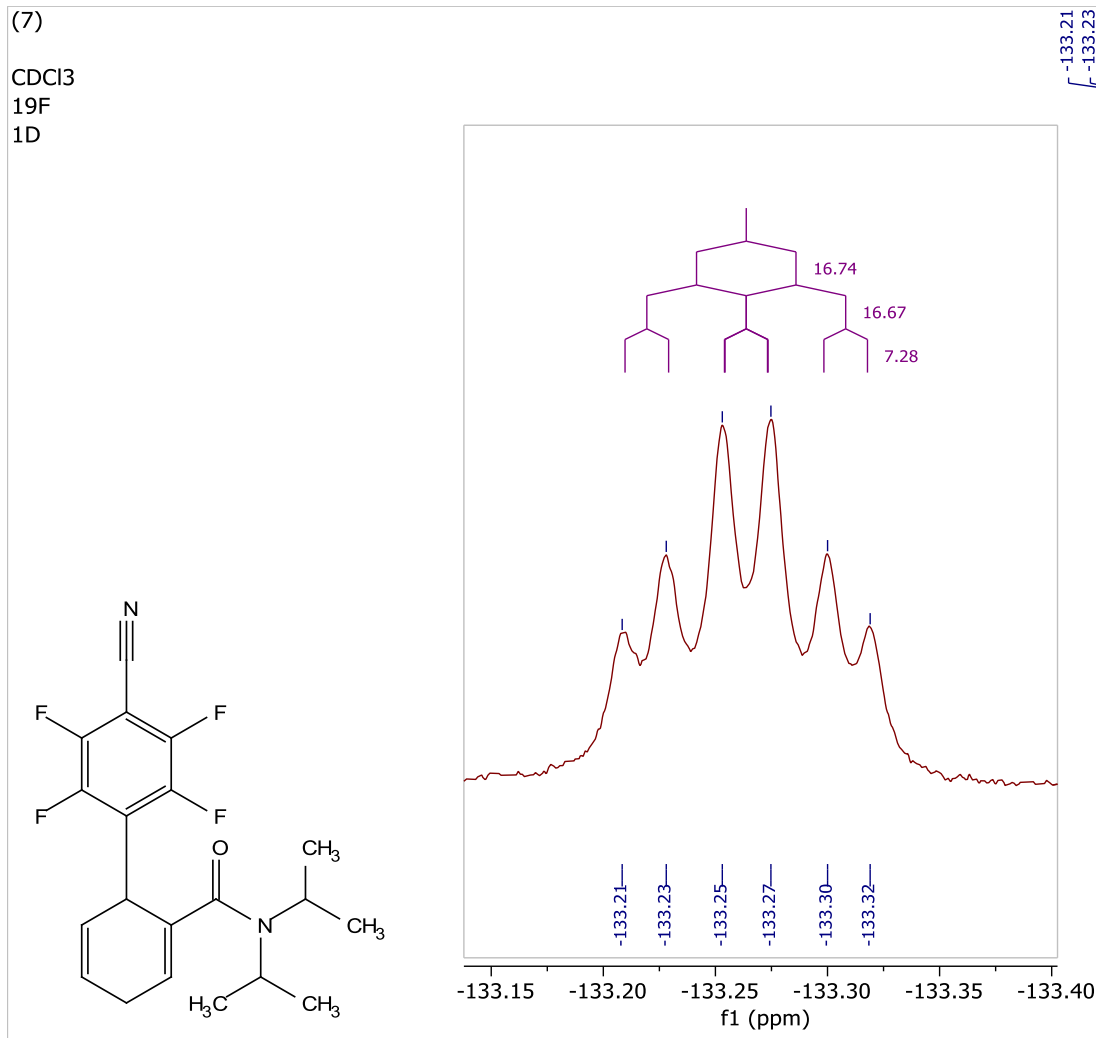

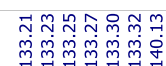

난
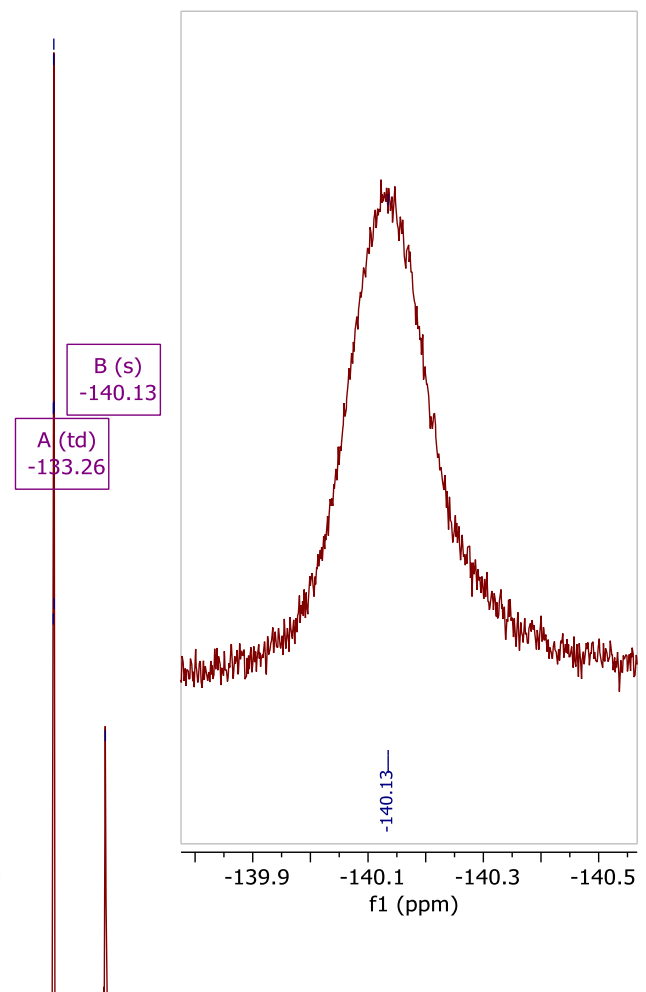

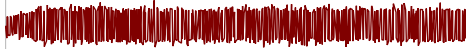

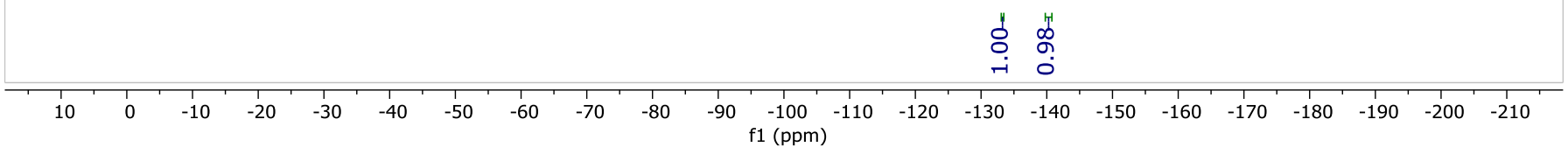


${ }^{1} \mathbf{H}$ NMR $\left(\mathrm{CD}_{3} \mathrm{CN}, 400 \mathrm{MHz}\right)$

(7)

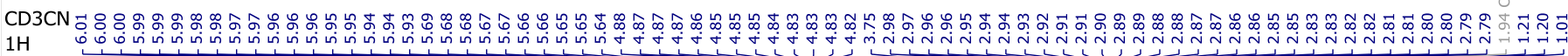

$1 \mathrm{H}$

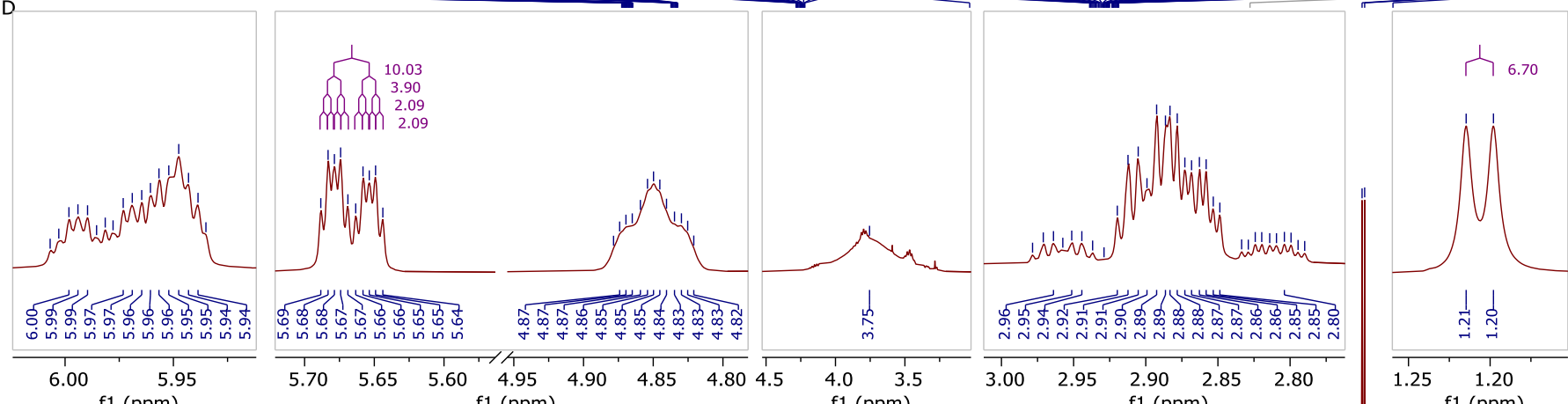


${ }^{13} \mathrm{C}\left\{{ }^{1} \mathrm{H}\right\}$ NMR $\left(\mathrm{CD}_{3} \mathrm{CN}, 101 \mathrm{MHz}\right)$

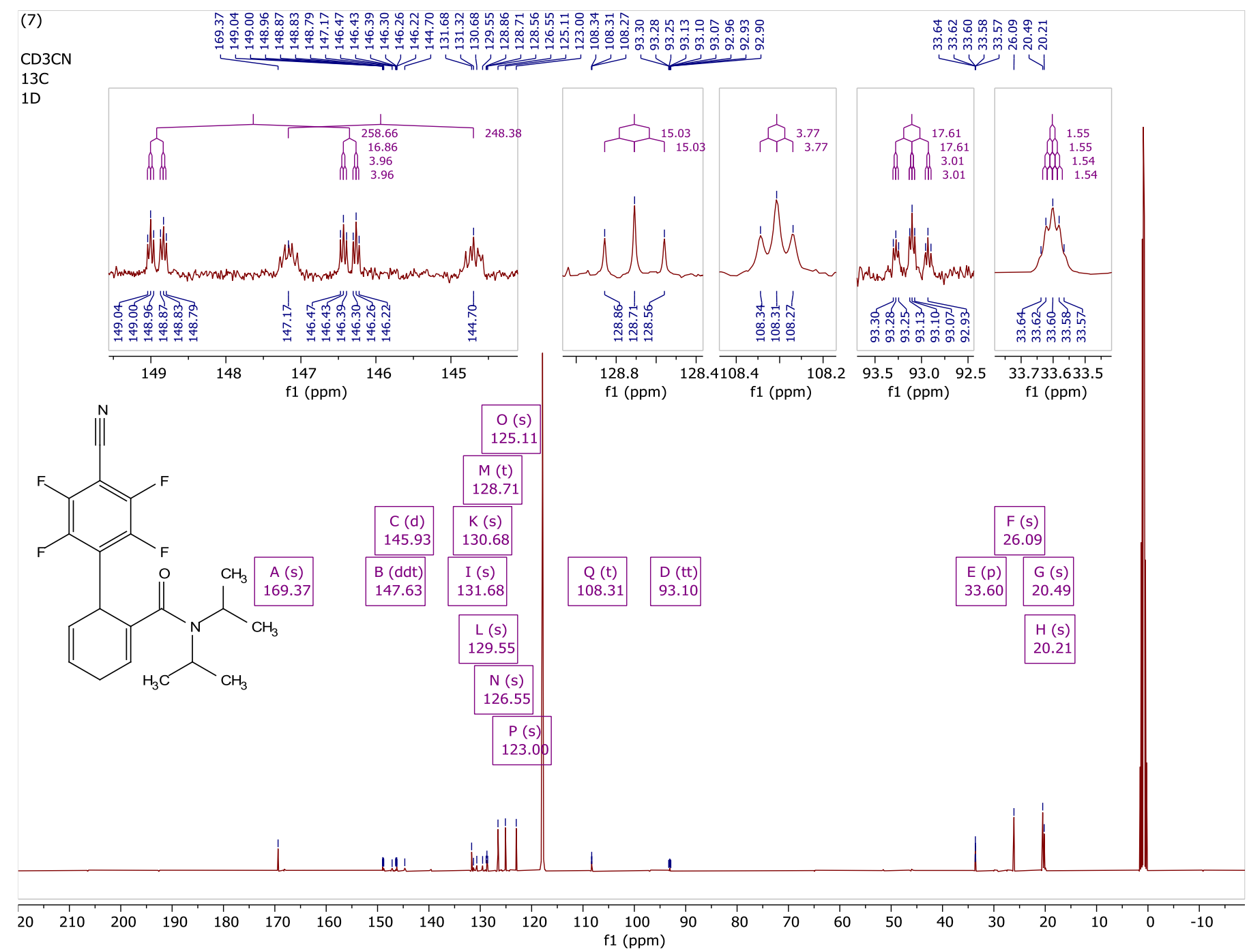


(8) 1-(4-methyl-6-(perfluoropyridin-4-yl)cyclohexa-1,4-dien-1-yl)ethan-1-one

${ }^{19} \mathrm{~F}$ NMR $\left(\mathrm{CDCl}_{3}, 376 \mathrm{MHz}\right)$

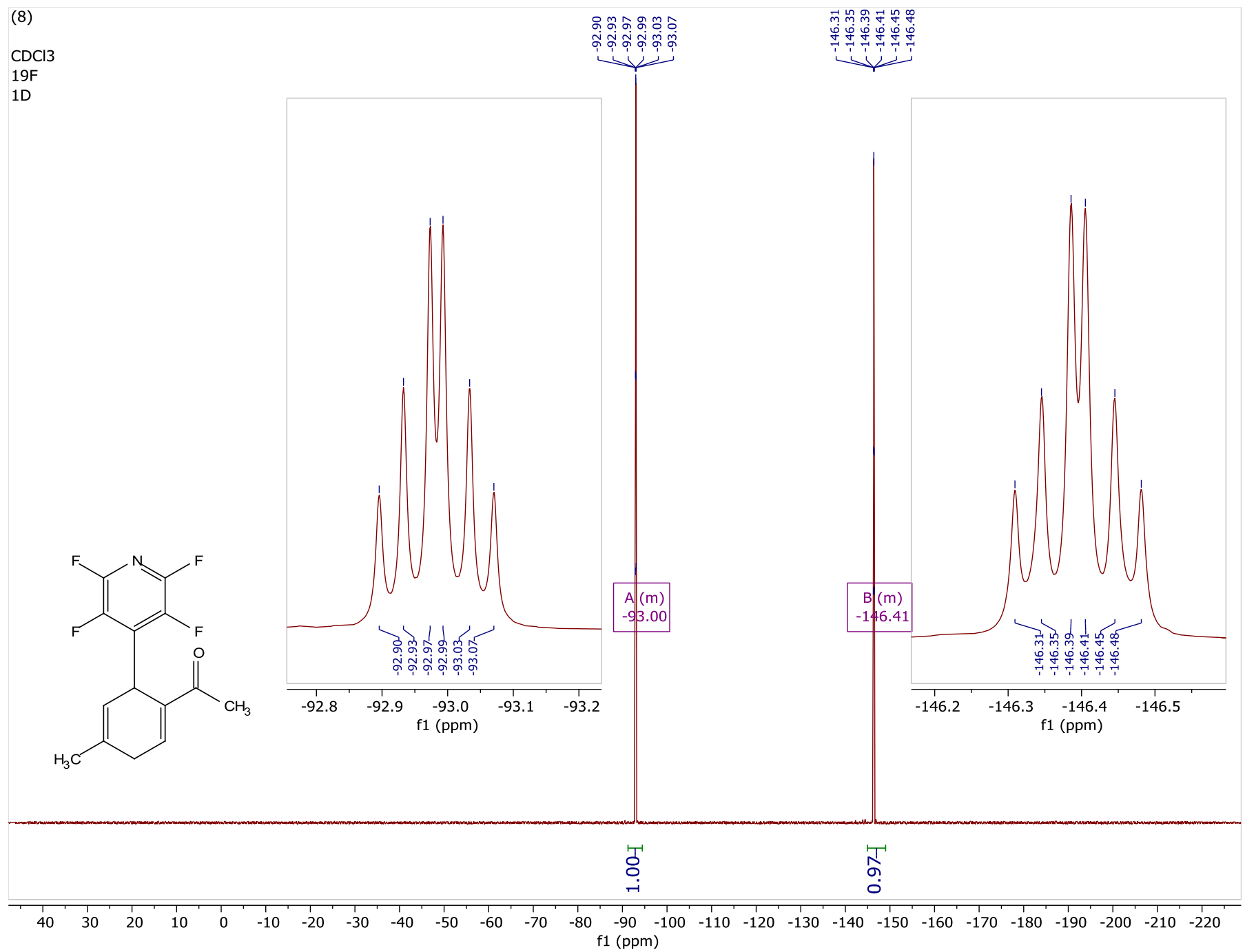


${ }^{\mathbf{1}} \mathbf{H}$ NMR $\left(\mathrm{CDCl}_{3}, 400 \mathrm{MHz}\right)$

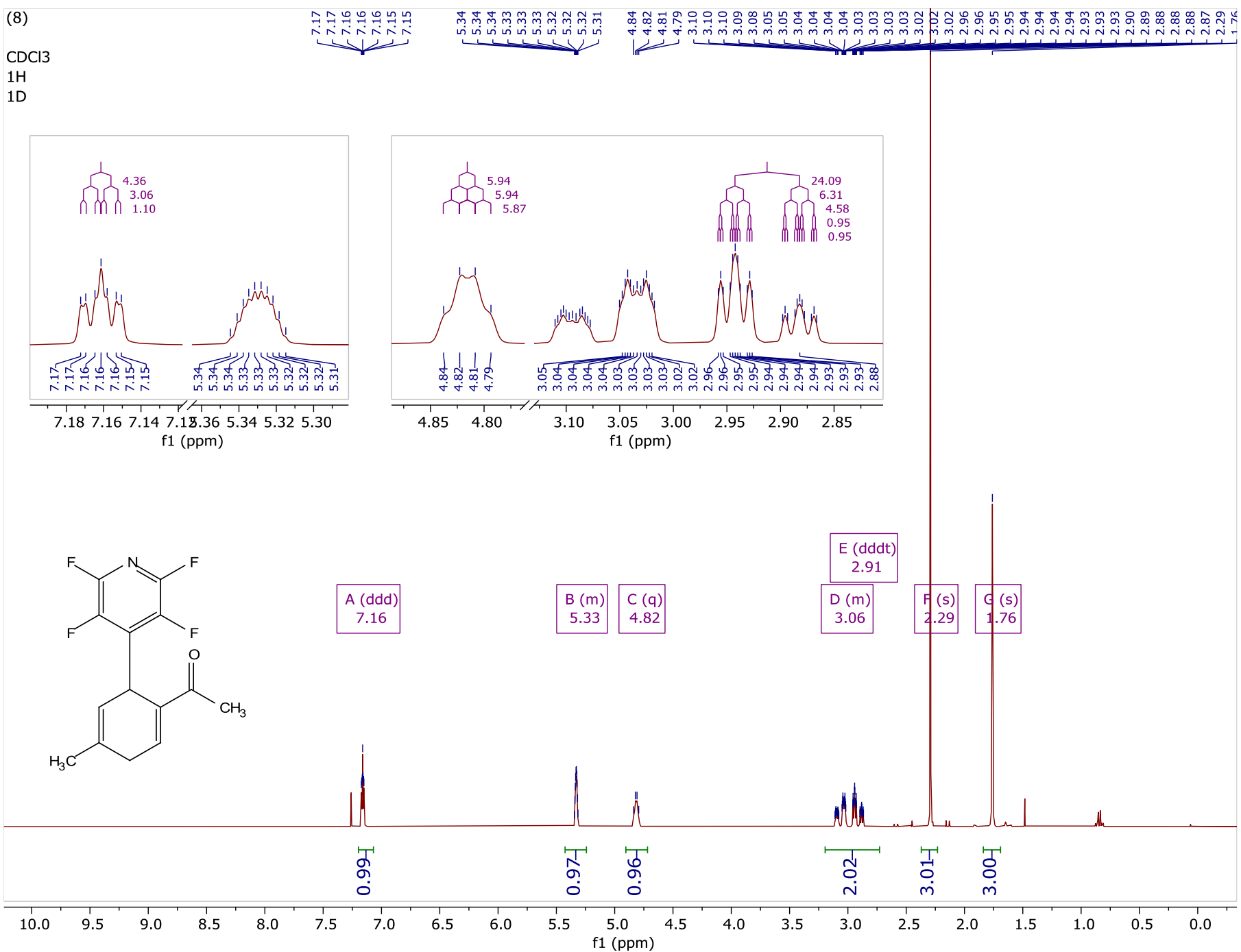


${ }^{13} \mathbf{C}\left\{{ }^{1} \mathrm{H}\right\}$ NMR $\left(\mathrm{CDCl}_{3}, 101 \mathrm{MHz}\right)$

(8)

$\mathrm{CDCl} 3$

$13 \mathrm{C}$

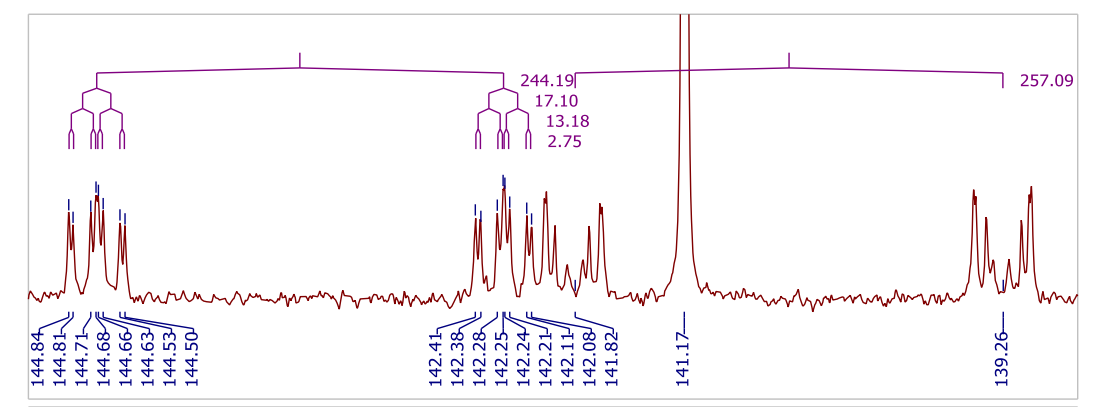

$\begin{array}{lllllllllllll}145.0 & 144.5 & 144.0 & 143.5 & 143.0 & 142.5 & 142.0 & 141.5 & 141.0 & 140.5 & 140.0 & 139.5 & 139.0\end{array}$ f1 (ppm)

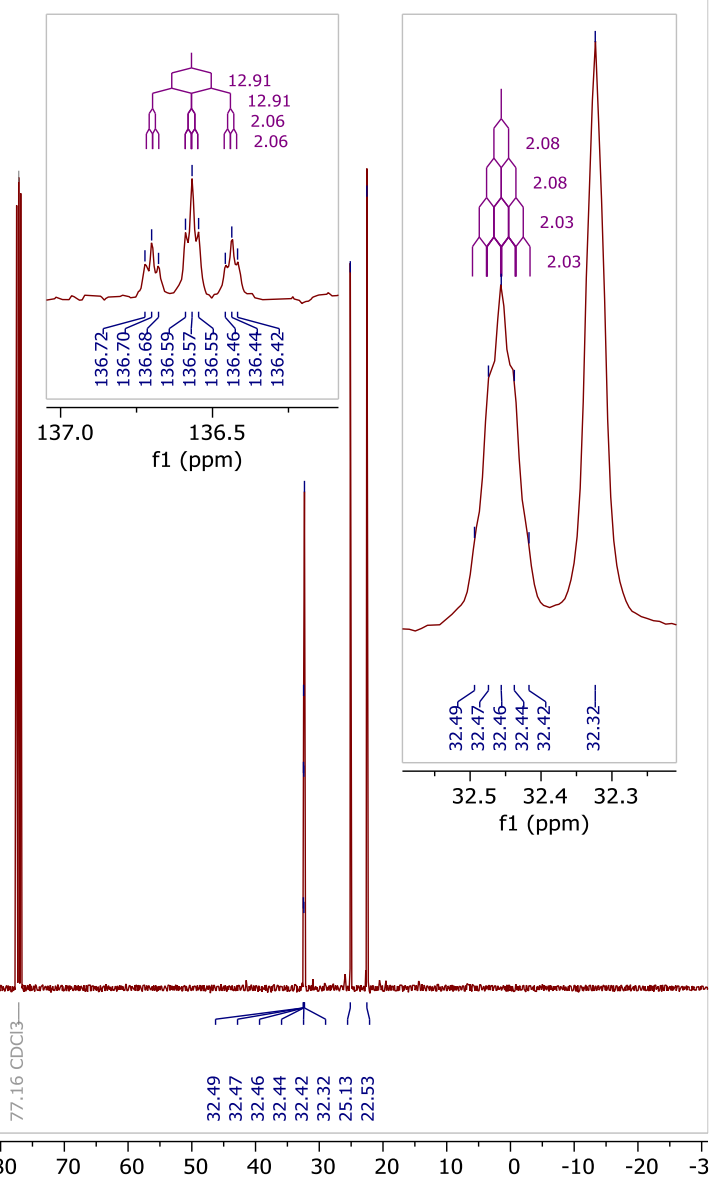

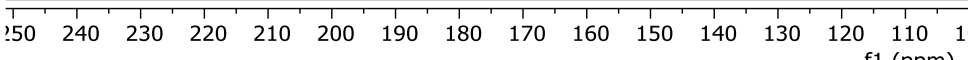


${ }^{1} \mathrm{H}-{ }^{13} \mathrm{C}$ HSQC

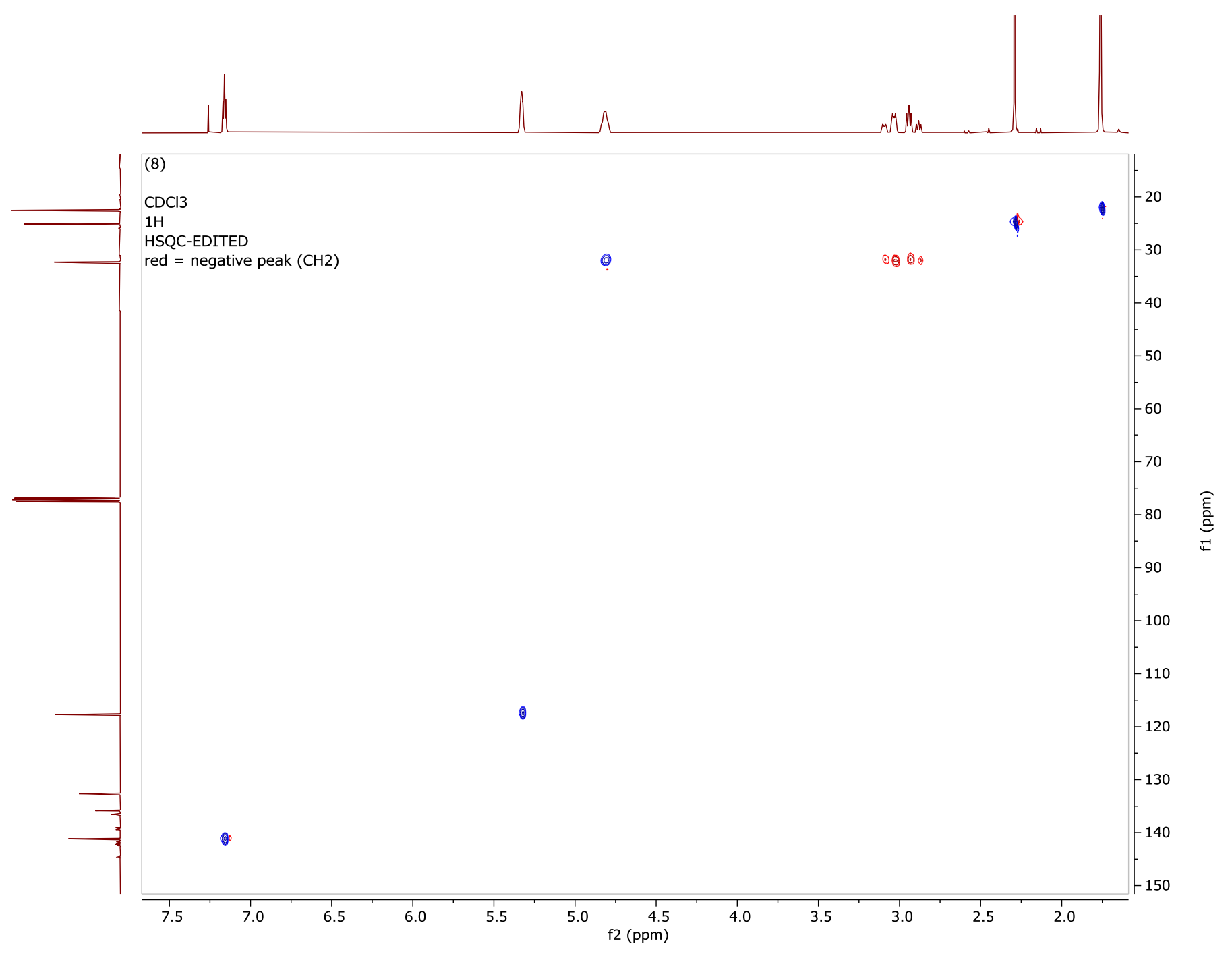




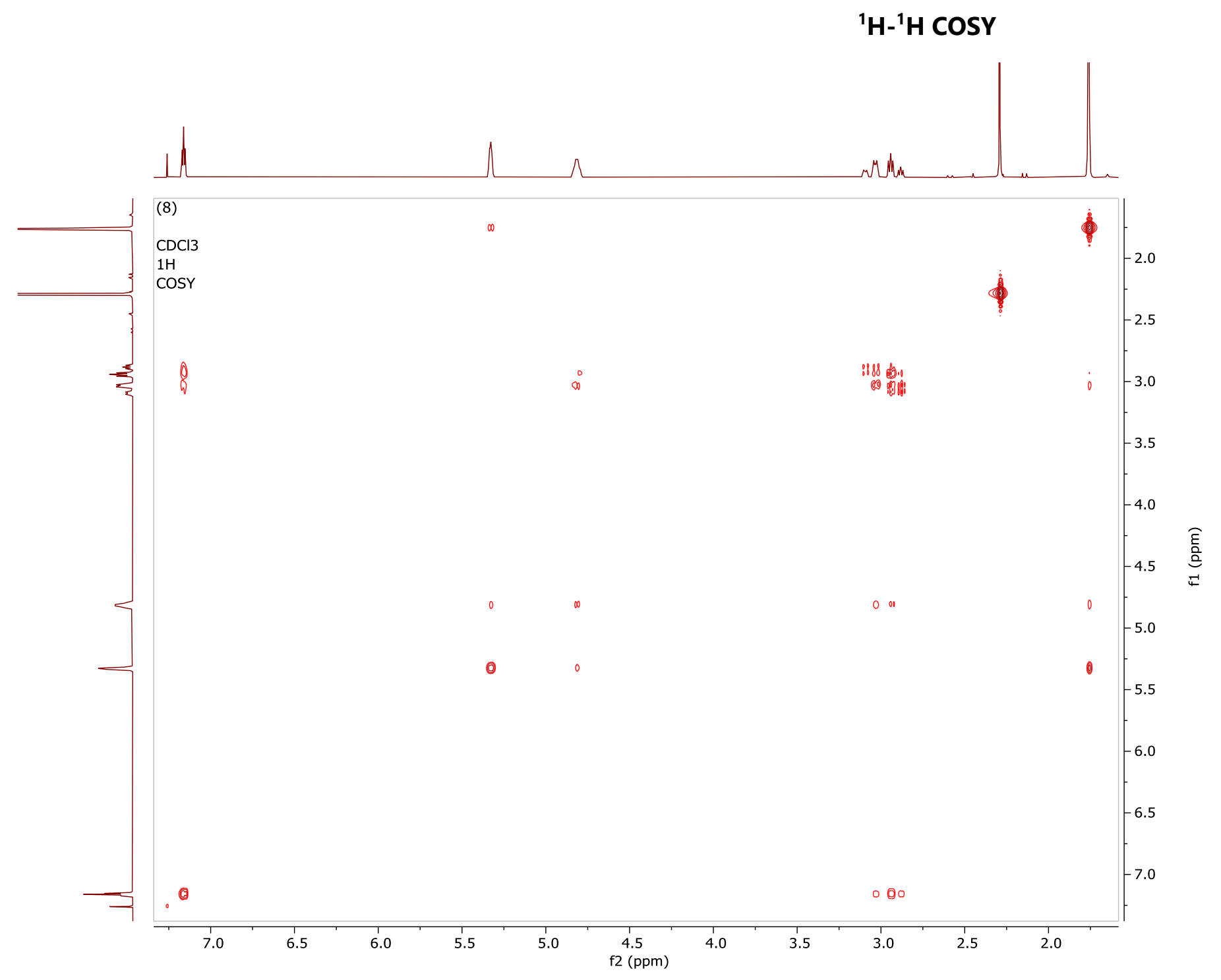


(9) methyl 4' -cyano-2',3',5',6' -tetrafluoro-1,4-dihydro-[1,1'-biphenyl]-2-carboxylate.

${ }^{19} \mathrm{~F}$ NMR $\left(\mathrm{CD}_{3} \mathrm{CN}, 376 \mathrm{MHz}\right)$

(9)

CD3CN

$19 F$

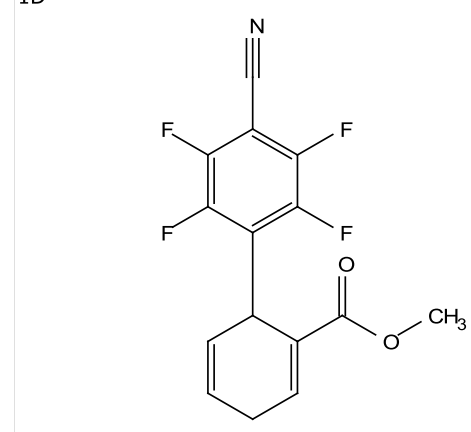

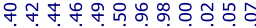

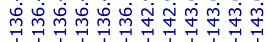

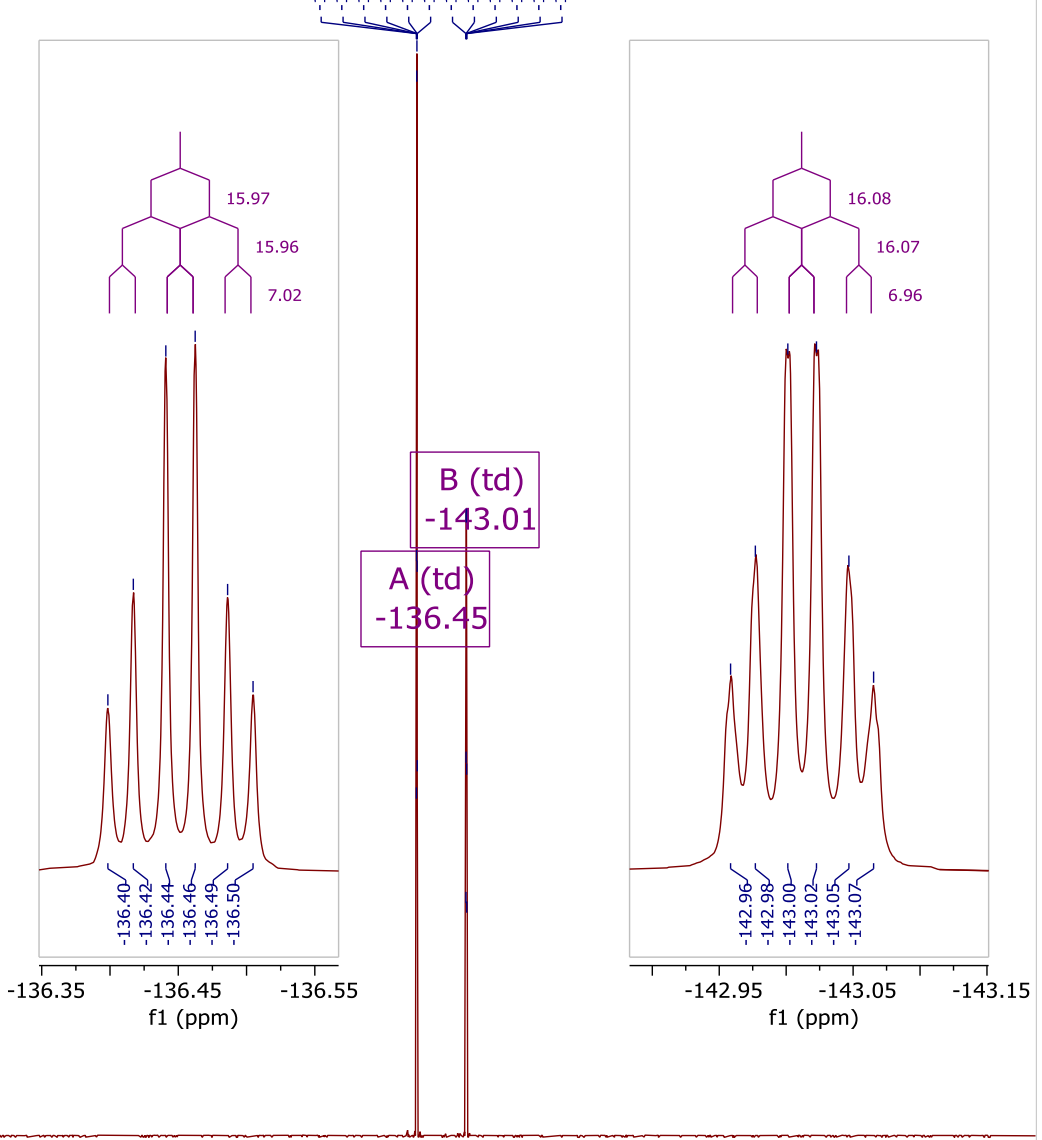

ถ้ ถู่

. 
${ }^{1} \mathrm{H}$ NMR $\left(\mathrm{CD}_{3} \mathrm{CN}, 400 \mathrm{MHz}\right)$

(9)

CD3CN

$1 \mathrm{H}$
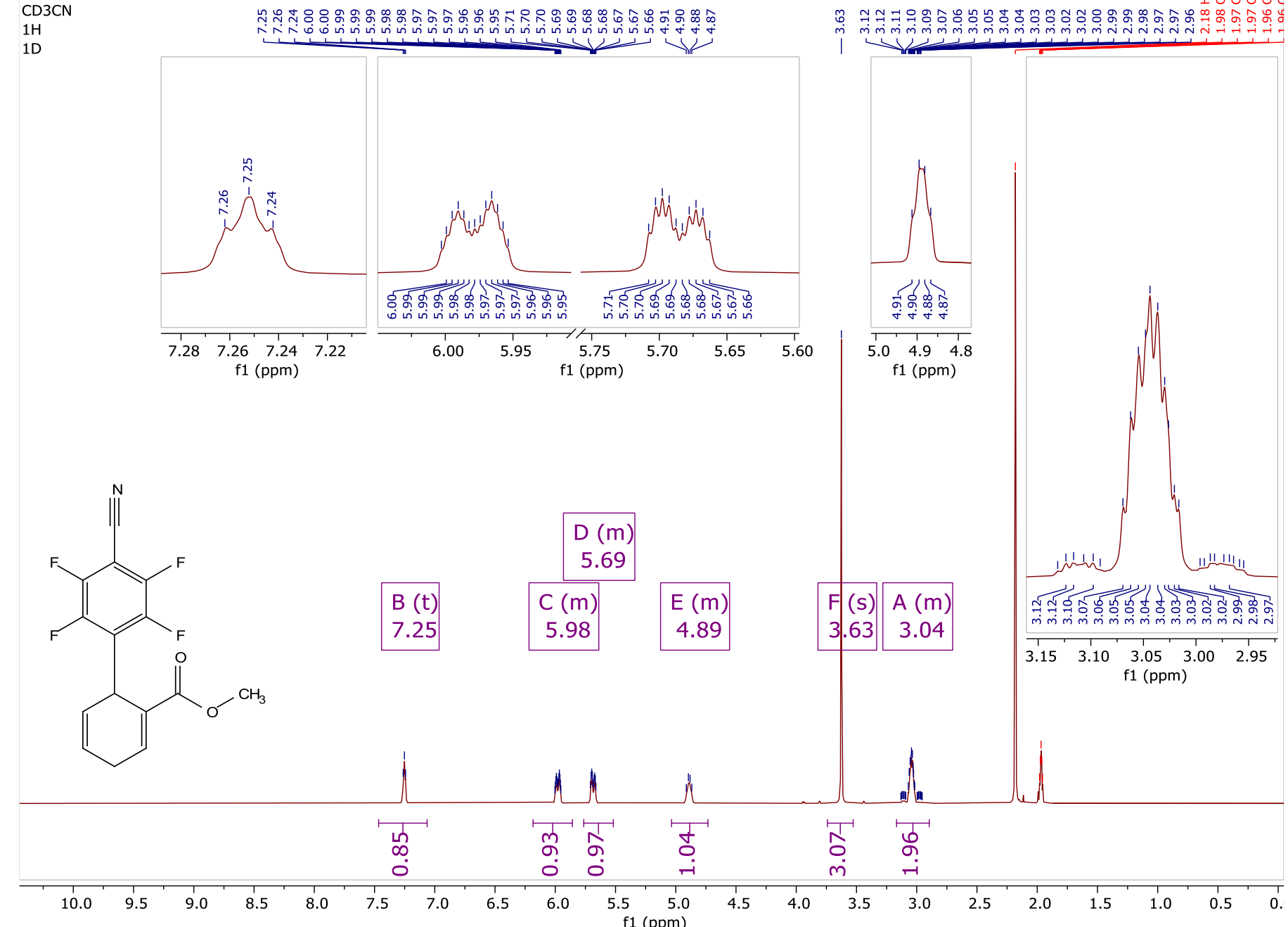

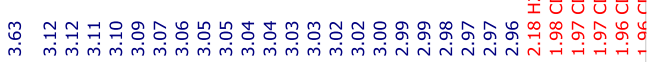

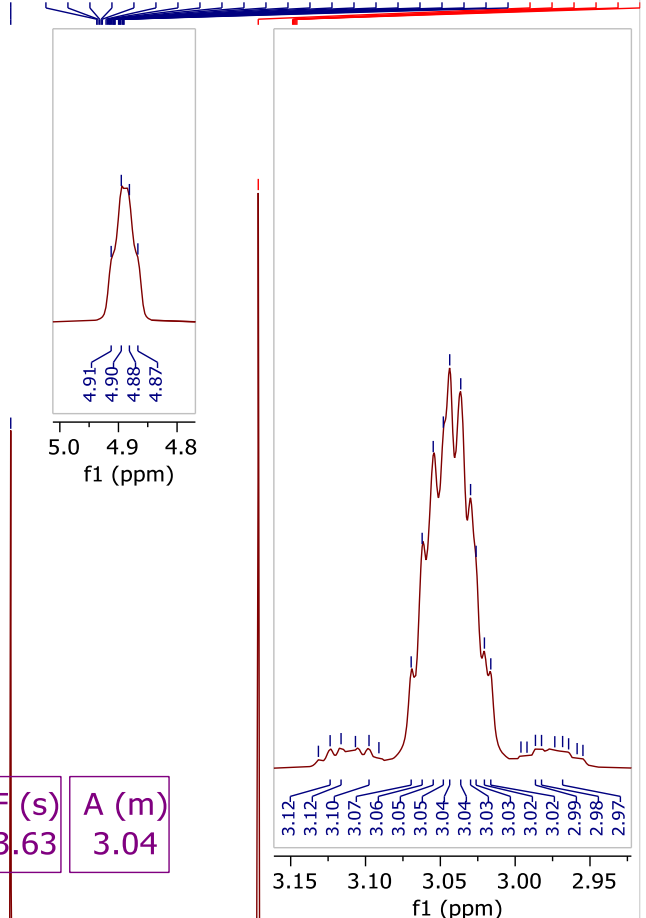


${ }^{13} \mathrm{C}\left\{{ }^{1} \mathrm{H}\right\}$ NMR $\left(\mathrm{CD}_{3} \mathrm{CN}, 101 \mathrm{MHz}\right)$

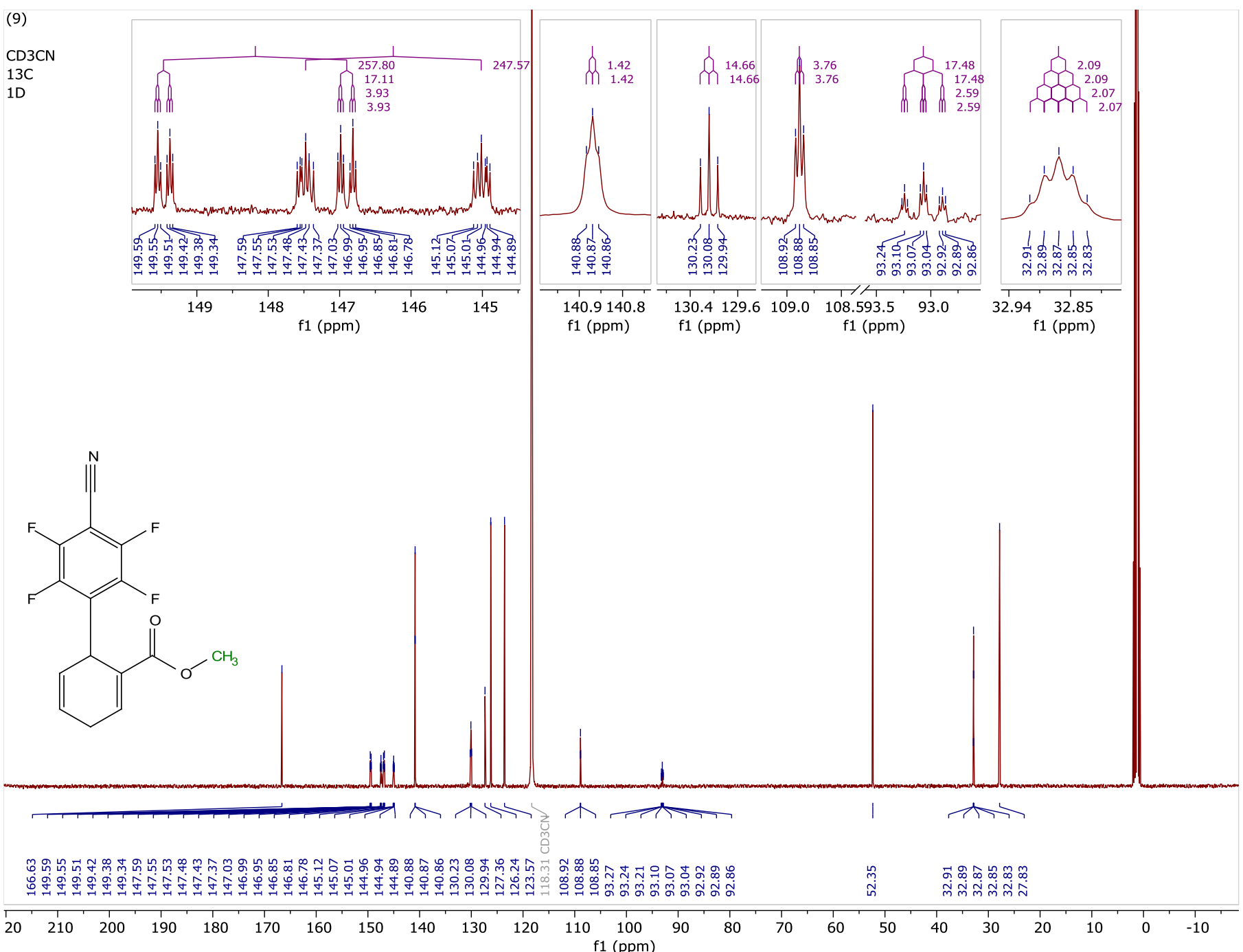


(10) 2 ',3',5',6' '-tetrafluoro-5-methyl-1,4-dihydro-[1,1'-biphenyl]-2,4' -dicarbonitrile.

${ }^{19}$ F NMR $\left(\mathrm{CDCl}_{3}, 376 \mathrm{MHz}\right)$

(10)

$\mathrm{CDCl} 3$

$19 \mathrm{~F}$

1D
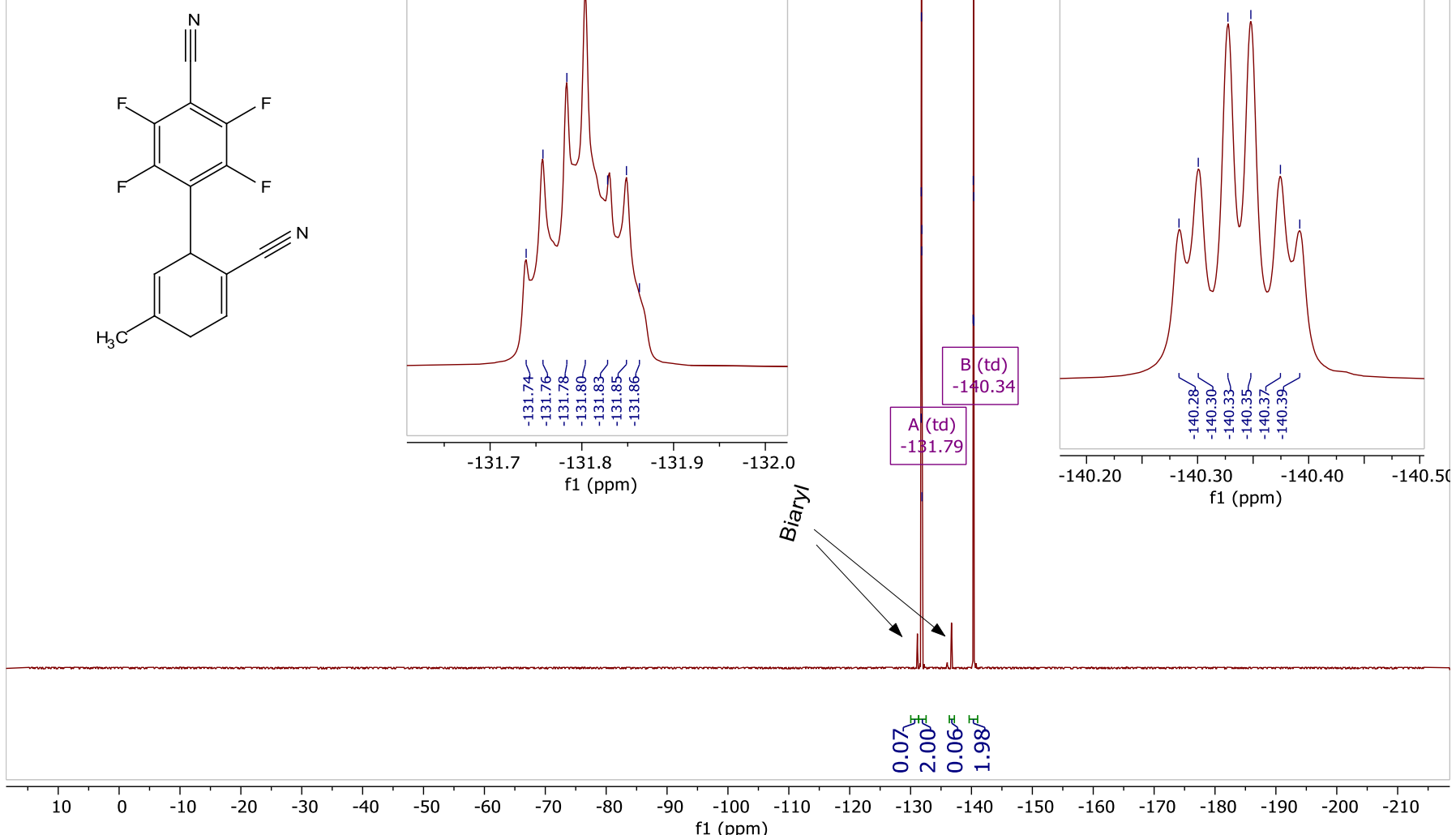
${ }^{1} \mathbf{H}$ NMR $\left(\mathrm{CDCl}_{3}, 400 \mathrm{MHz}\right)$

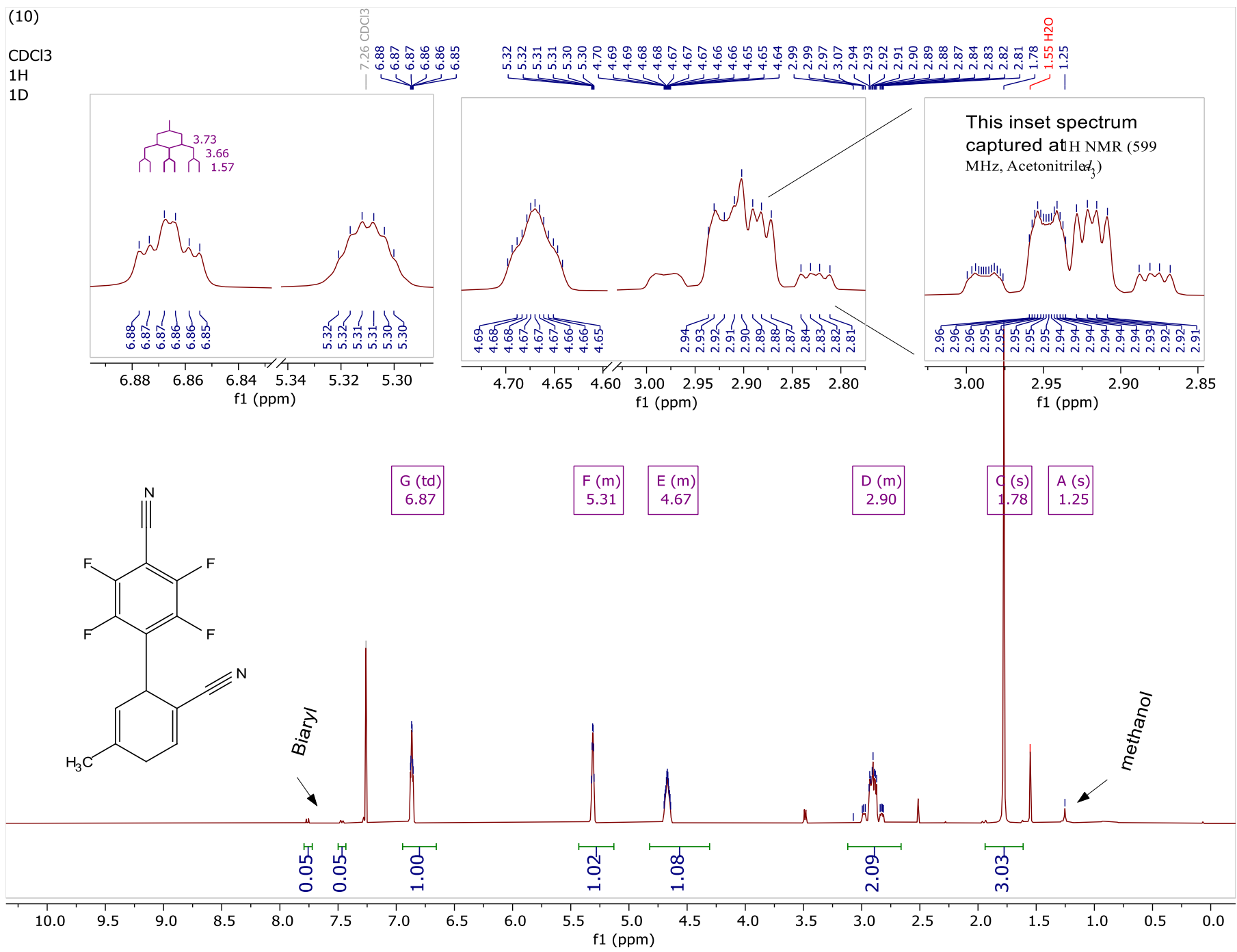


${ }^{13} \mathrm{C}\left\{{ }^{1} \mathrm{H}\right\}$ NMR $\left(\mathrm{CD}_{3} \mathrm{CN}, 101 \mathrm{MHz}\right)$

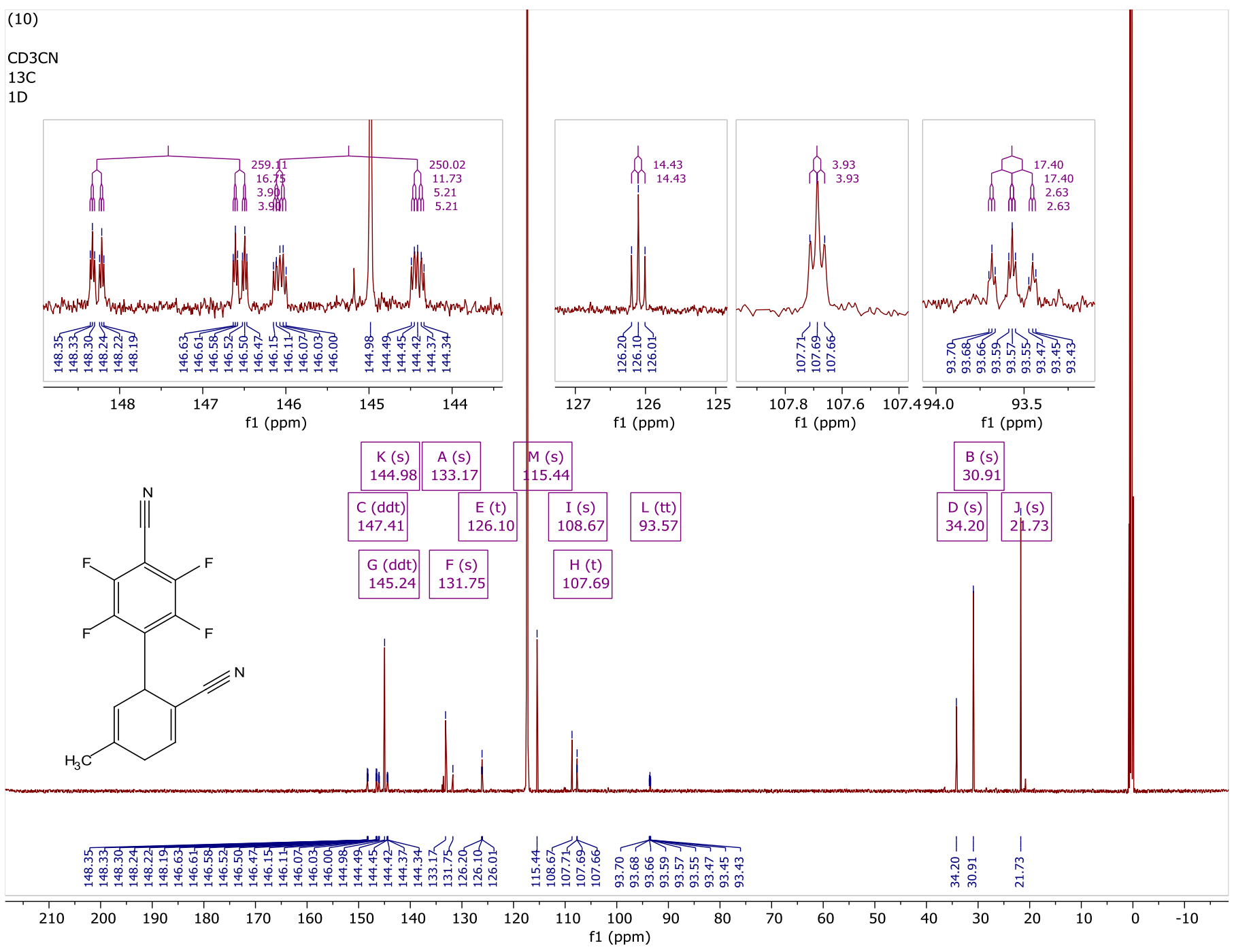


(11) methyl 5-((tert-butoxycarbonyl)oxy)-4'-cyano-2',3',5',6'-tetrafluoro-1,4-dihydro-[1,1'-biphenyl]-2-carboxylate.

${ }^{19}$ F NMR $\left(\mathrm{CDCl}_{3}, 376 \mathrm{MHz}\right)$

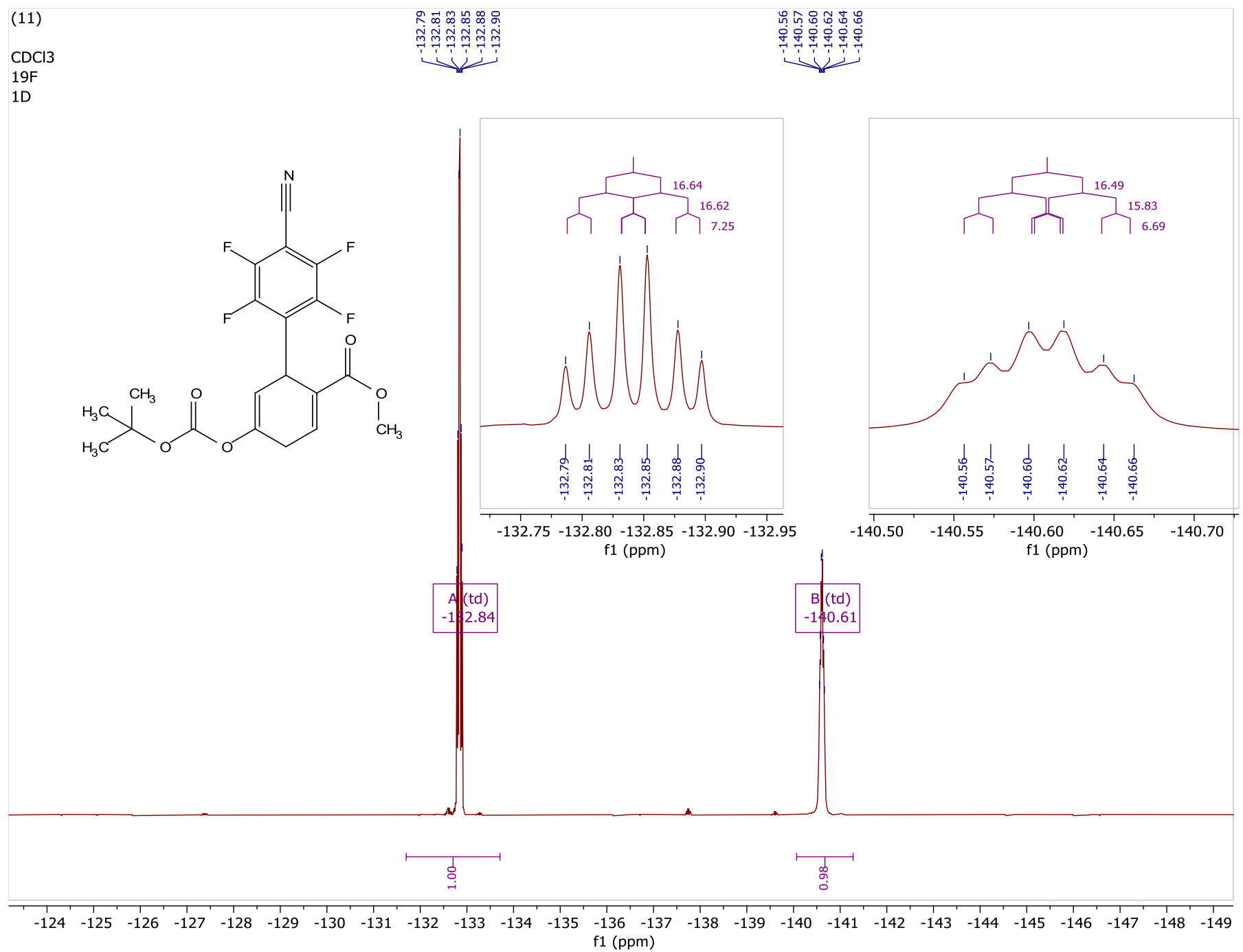


${ }^{\mathbf{1}} \mathbf{H}$ NMR $\left(\mathrm{CDCl}_{3}, 400 \mathrm{MHz}\right)$

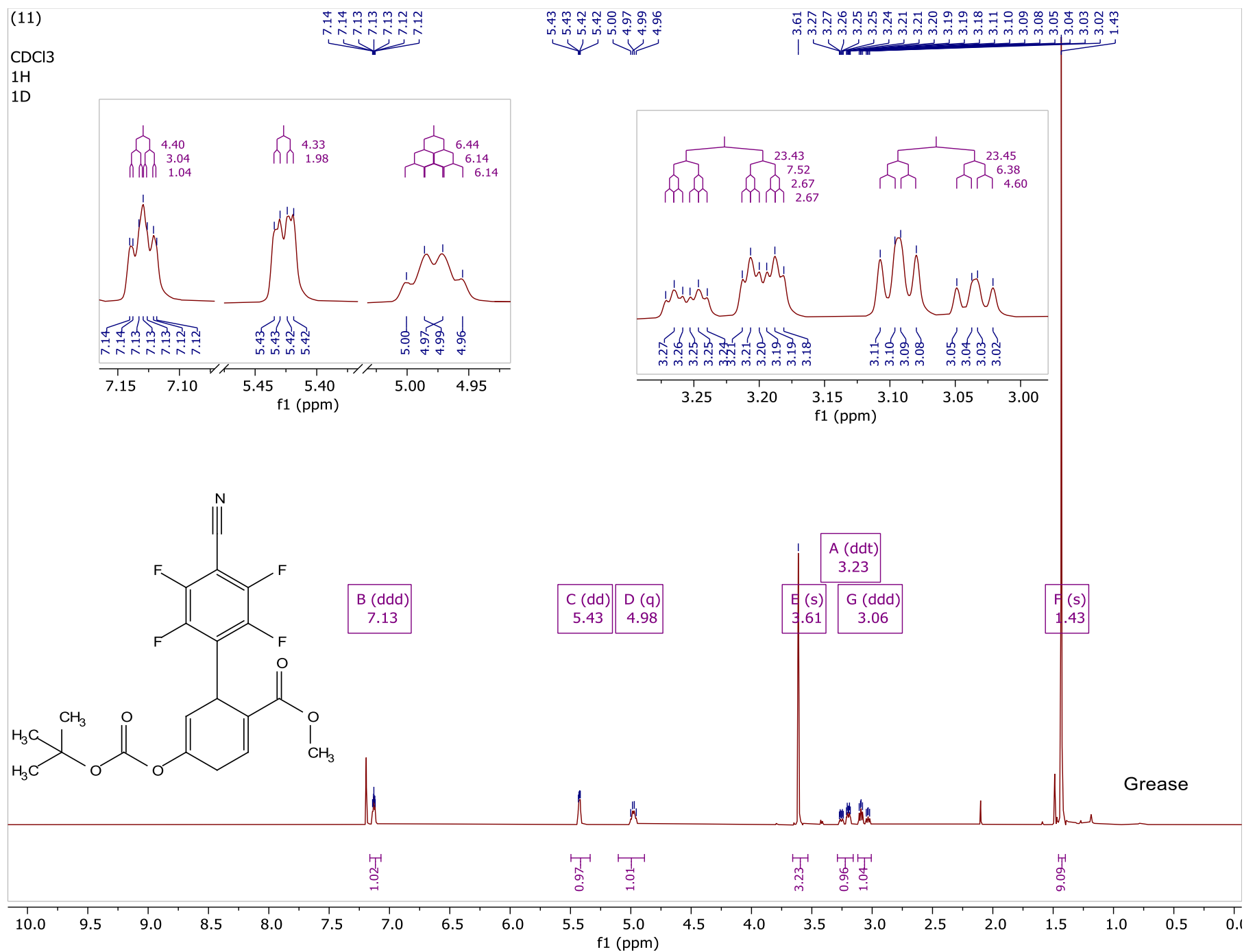


${ }^{13} \mathbf{C}\left\{{ }^{1} \mathbf{H}\right\}$ NMR $\left(\mathrm{CDCl}_{3}, 101 \mathrm{MHz}\right)$

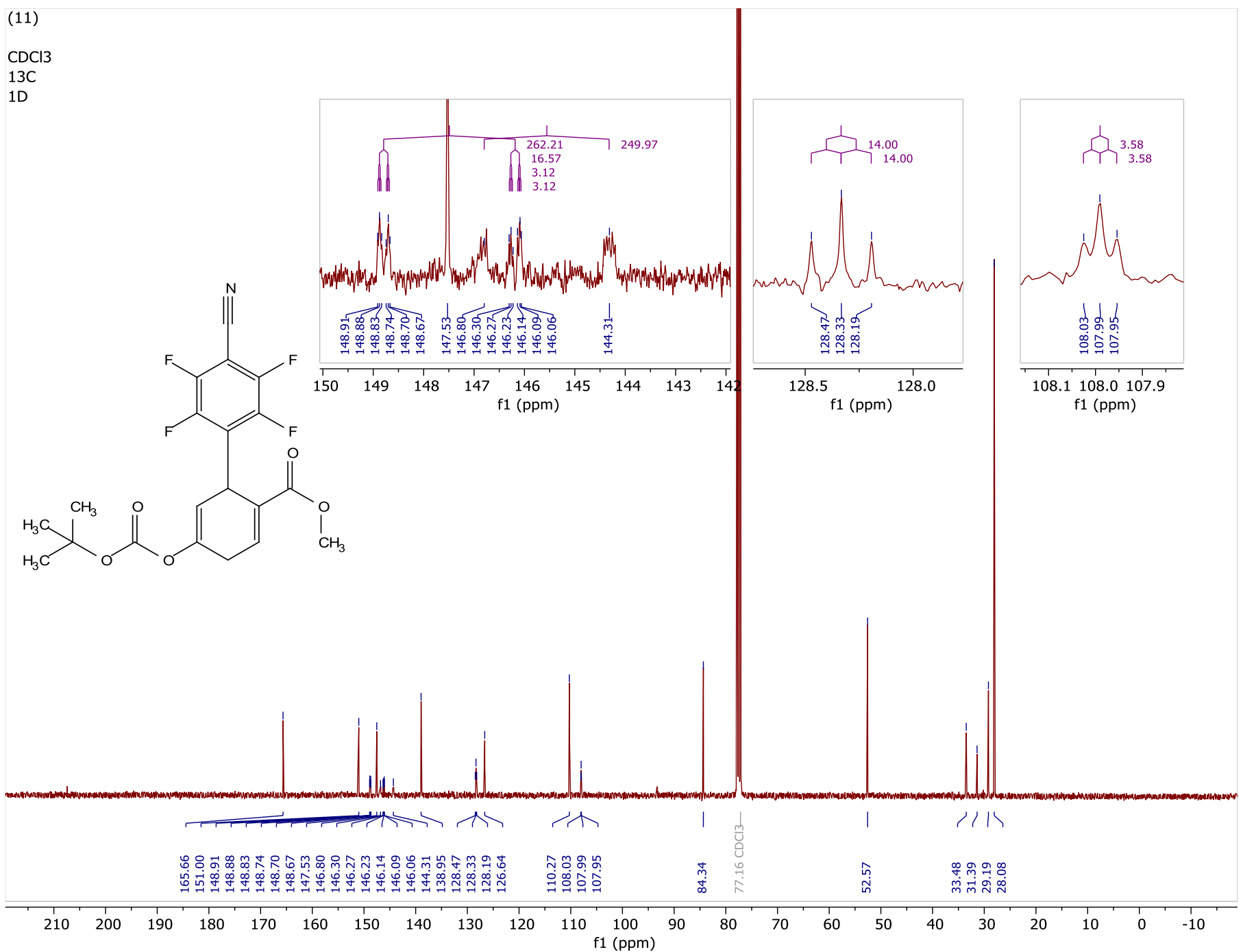


(12) (4-methyl-6-(perfluoropyridin-4-yl)cyclohexa-1,4-dien-1-yl)(piperidin-1-yl)methanone

${ }^{19}$ F NMR $\left(\mathrm{CDCl}_{3}, 376 \mathrm{MHz}\right)$

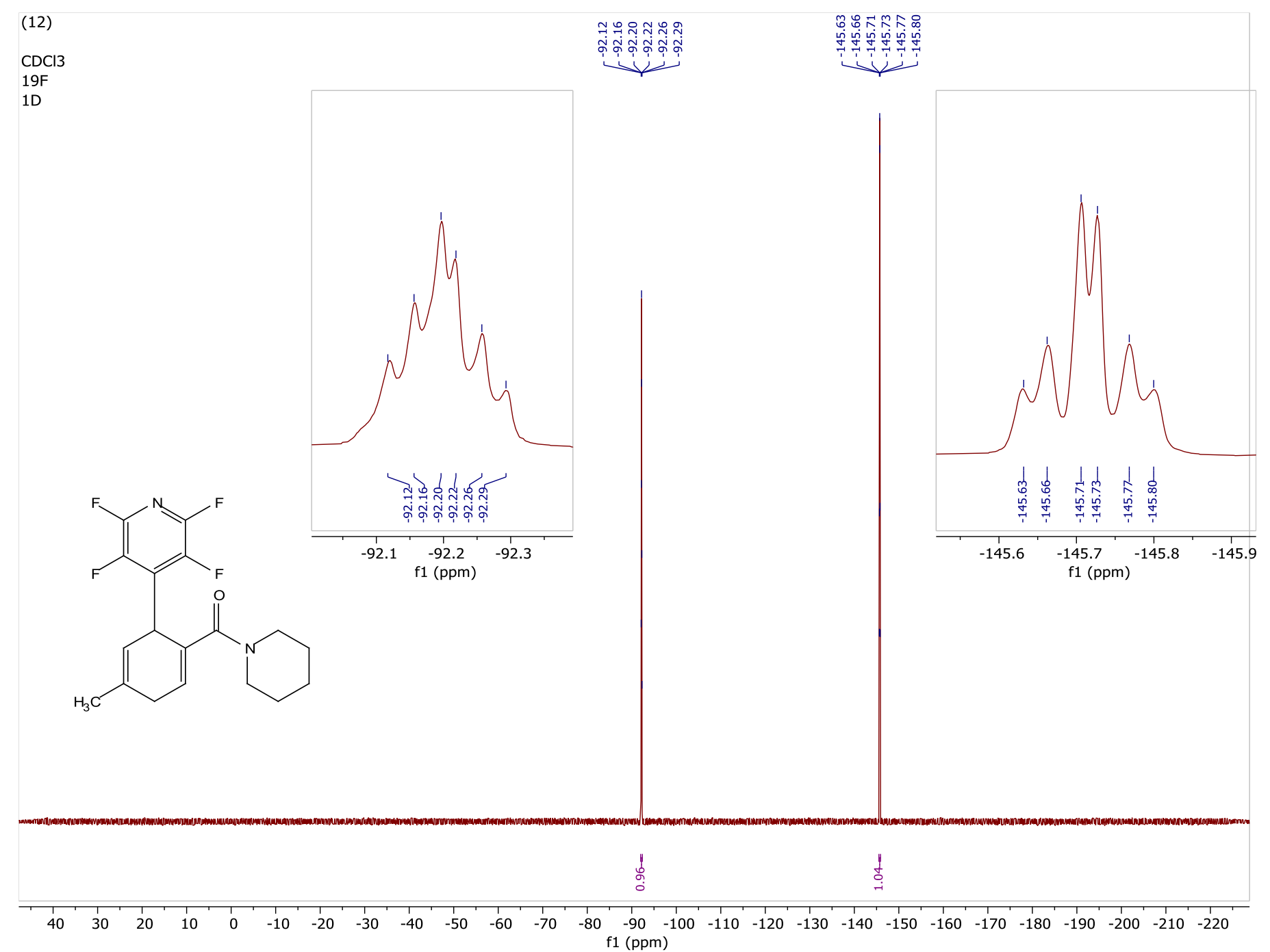


${ }^{\mathbf{1}} \mathbf{H} \mathbf{N M R}\left(\mathrm{CDCl}_{3}, 400 \mathrm{MHz}\right)$

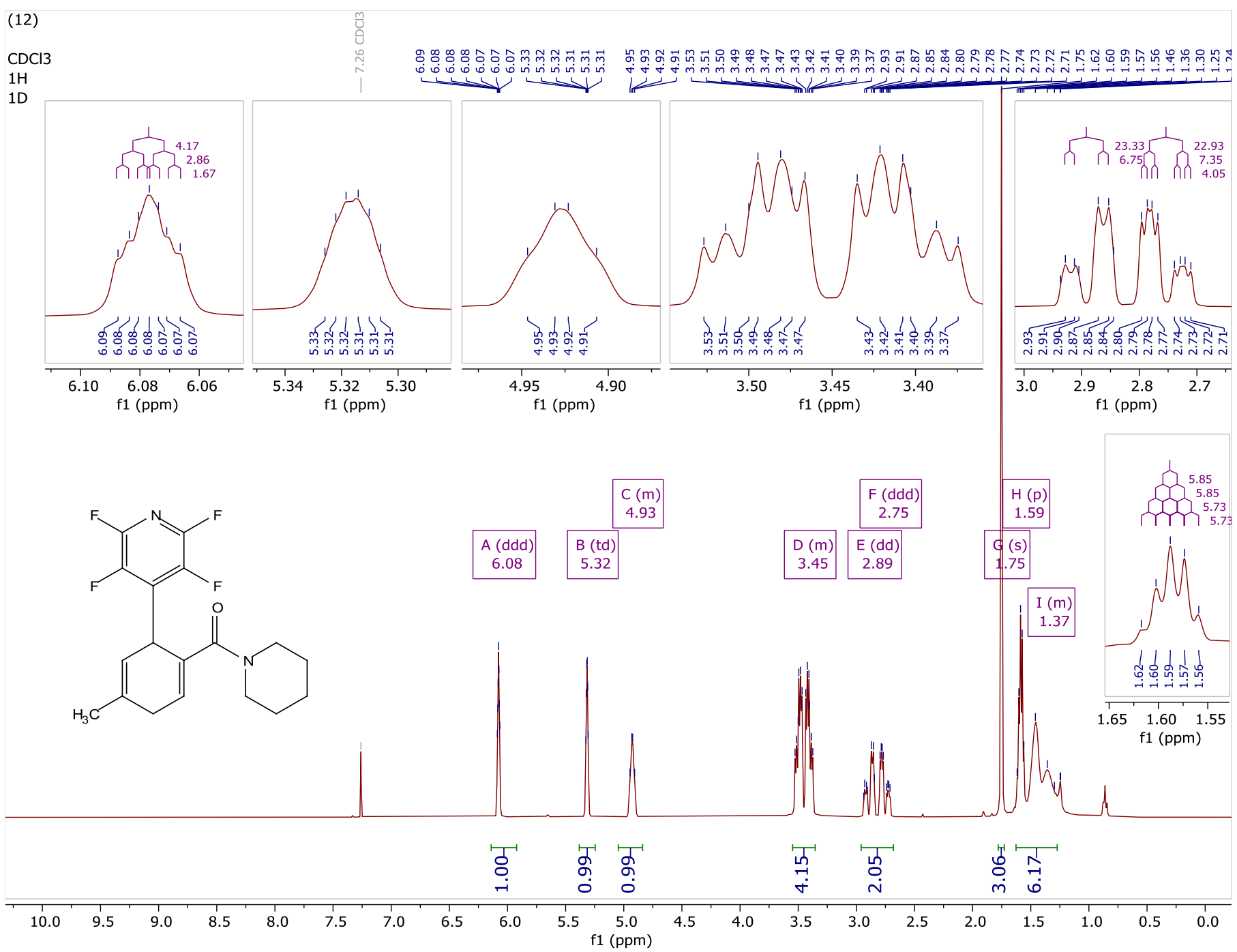




\section{${ }^{13} \mathrm{C}\left\{{ }^{1} \mathrm{H}\right\}$ NMR $\left(\mathrm{CD}_{3} \mathrm{CN}, 101 \mathrm{MHz}\right)$}

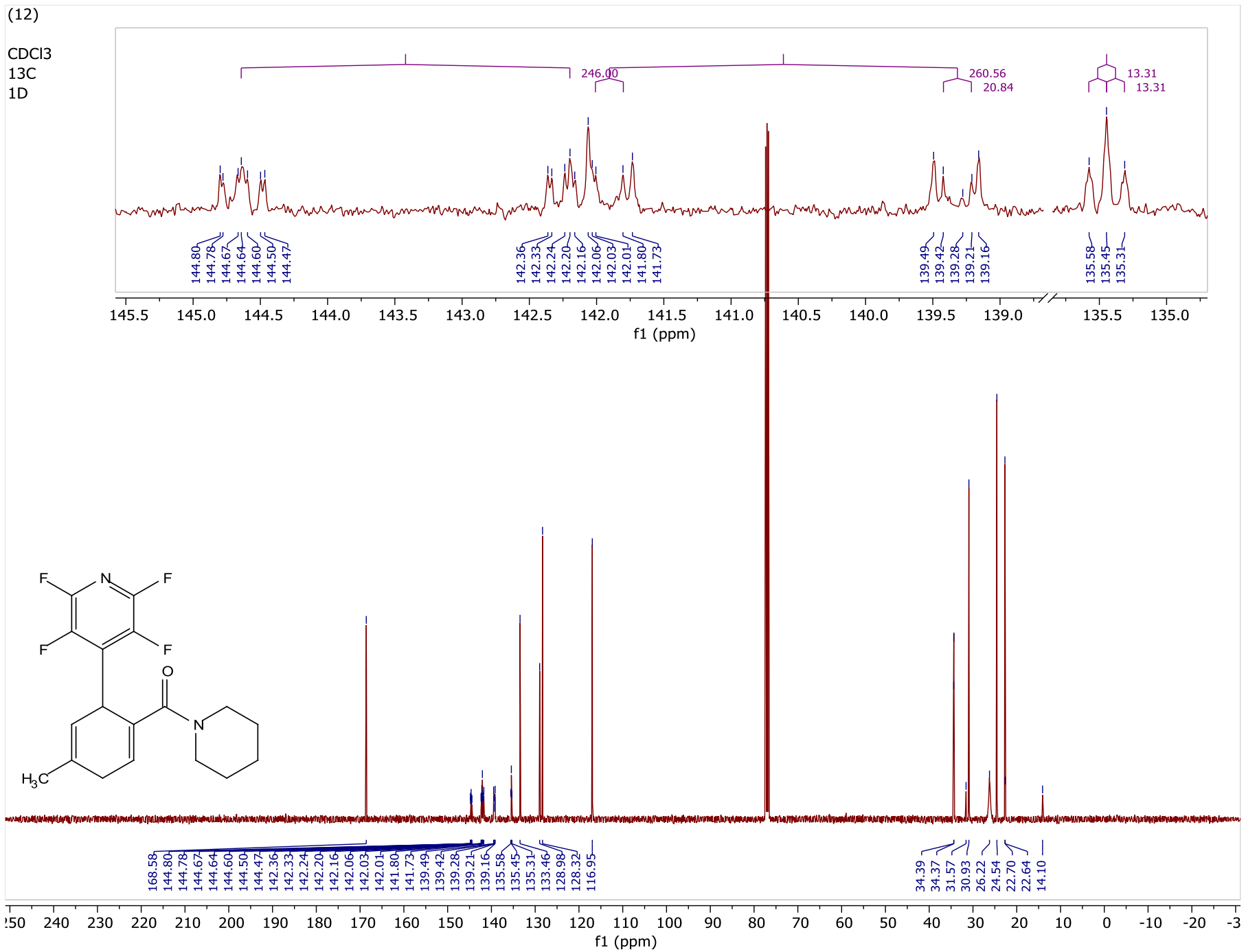


(13) dimethyl 3-(perfluoropyridin-4-yl)cyclohex-1-ene-1,4-dicarboxylate

${ }^{19}$ F NMR $\left(\mathrm{CDCl}_{3}, 376 \mathrm{MHz}\right)$

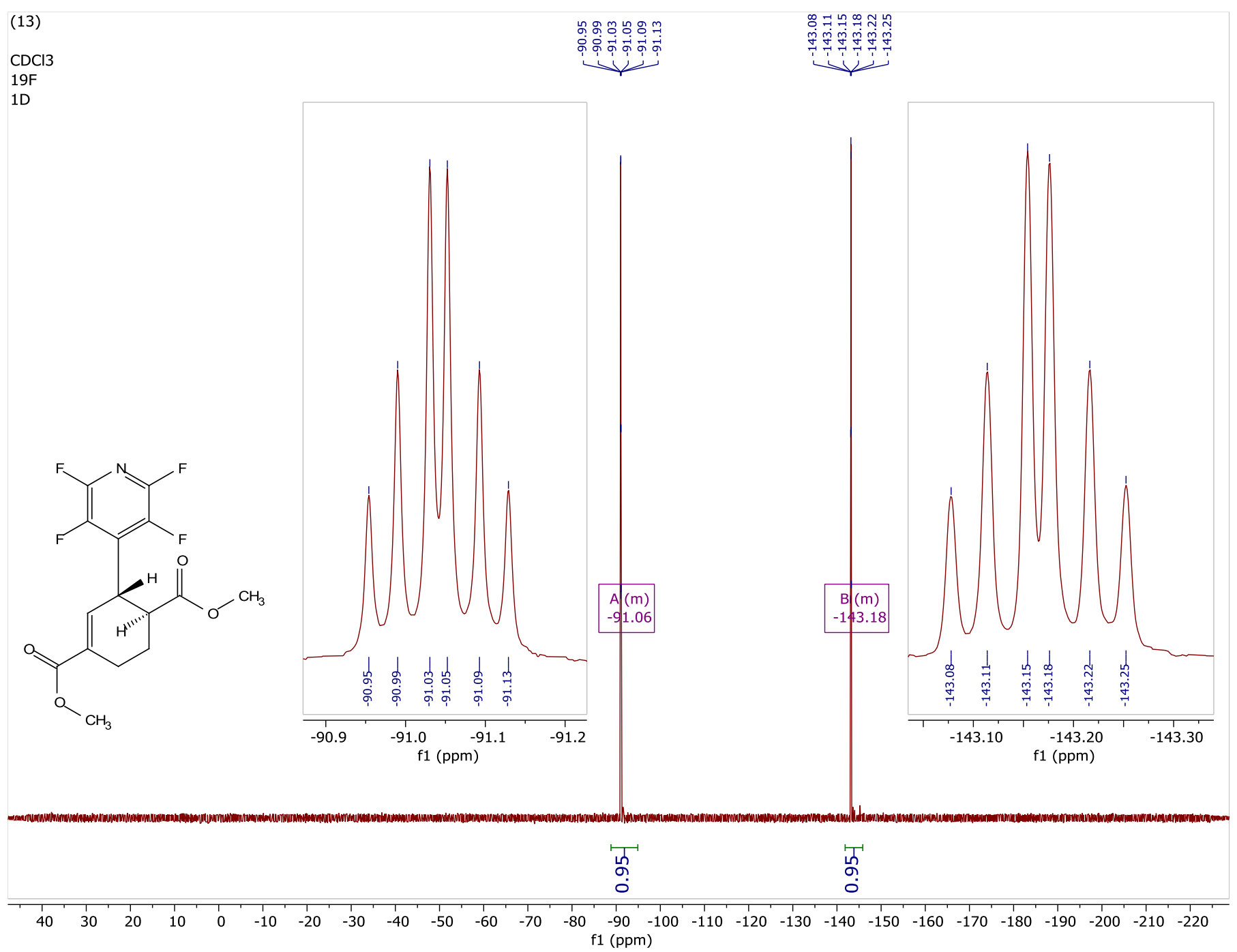


${ }^{1} \mathbf{H}$ NMR $\left(\mathrm{CDCl}_{3}, 400 \mathrm{MHz}\right)$

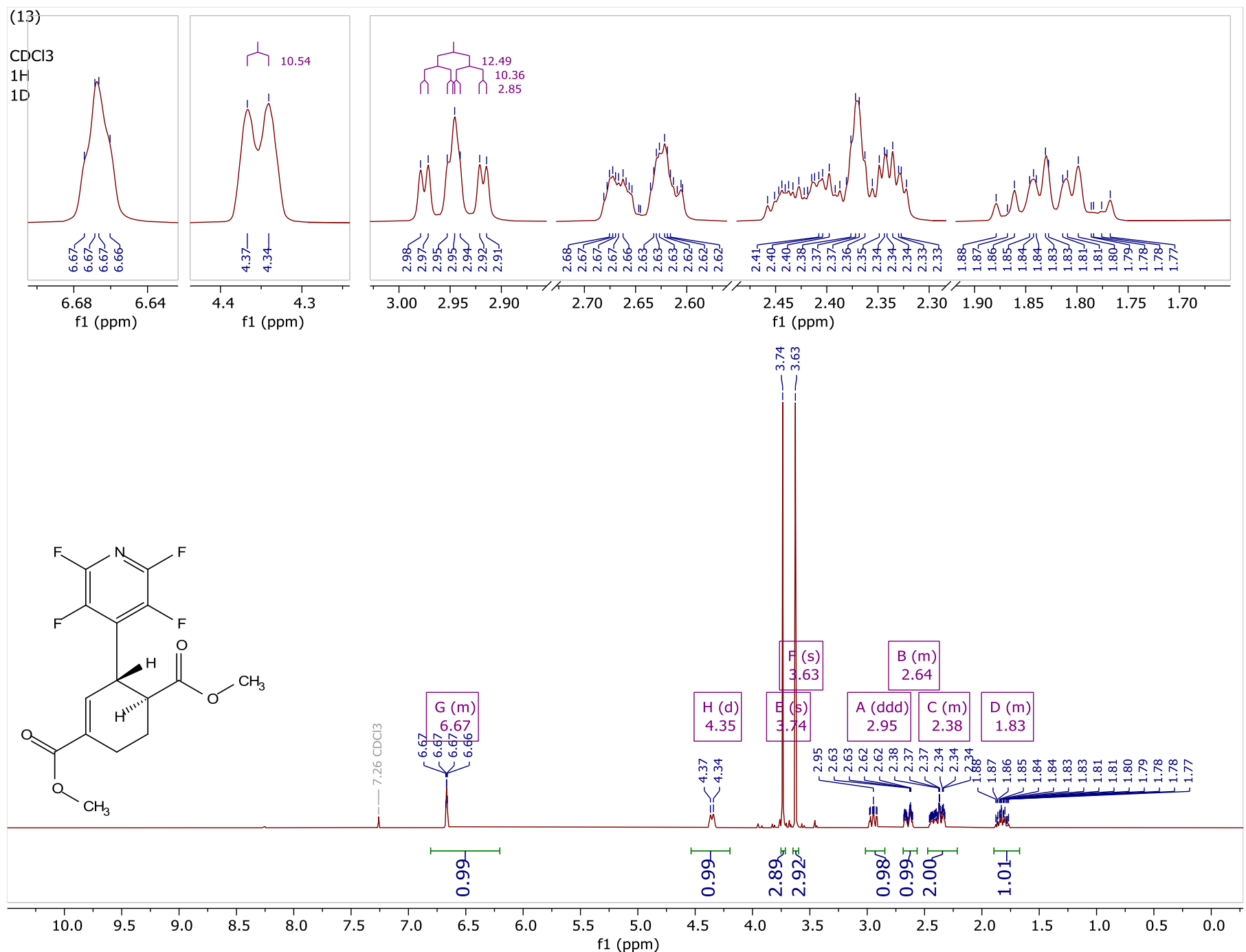




\section{${ }^{13} \mathrm{C}\left\{{ }^{1} \mathrm{H}\right\}$ NMR $\left(\mathrm{CD}_{3} \mathrm{CN}, 101 \mathrm{MHz}\right)$}

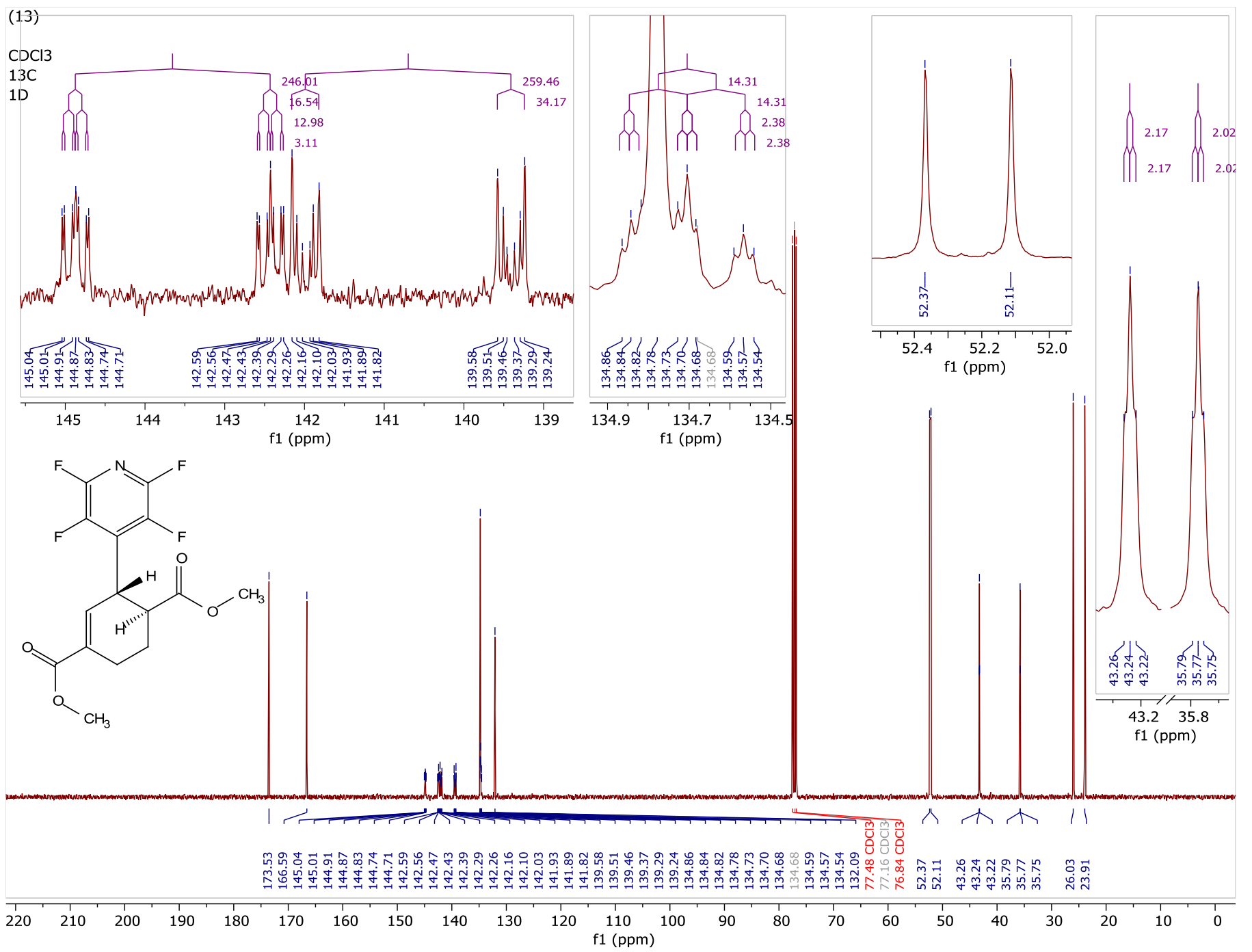


${ }^{1} \mathrm{H}-{ }^{13} \mathrm{C}$ HSQC

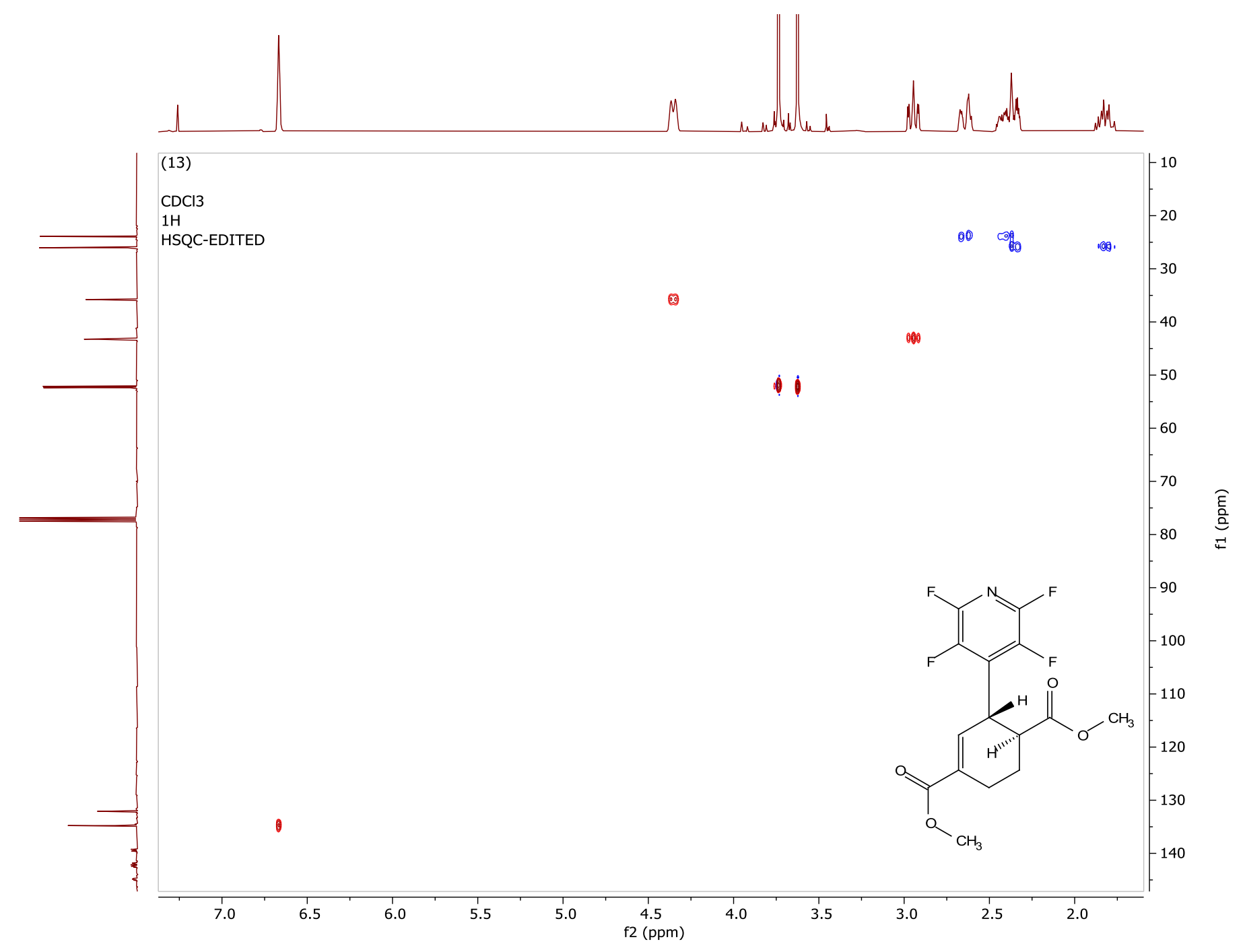


${ }^{1} \mathrm{H}-{ }^{1} \mathrm{H} \cos Y$

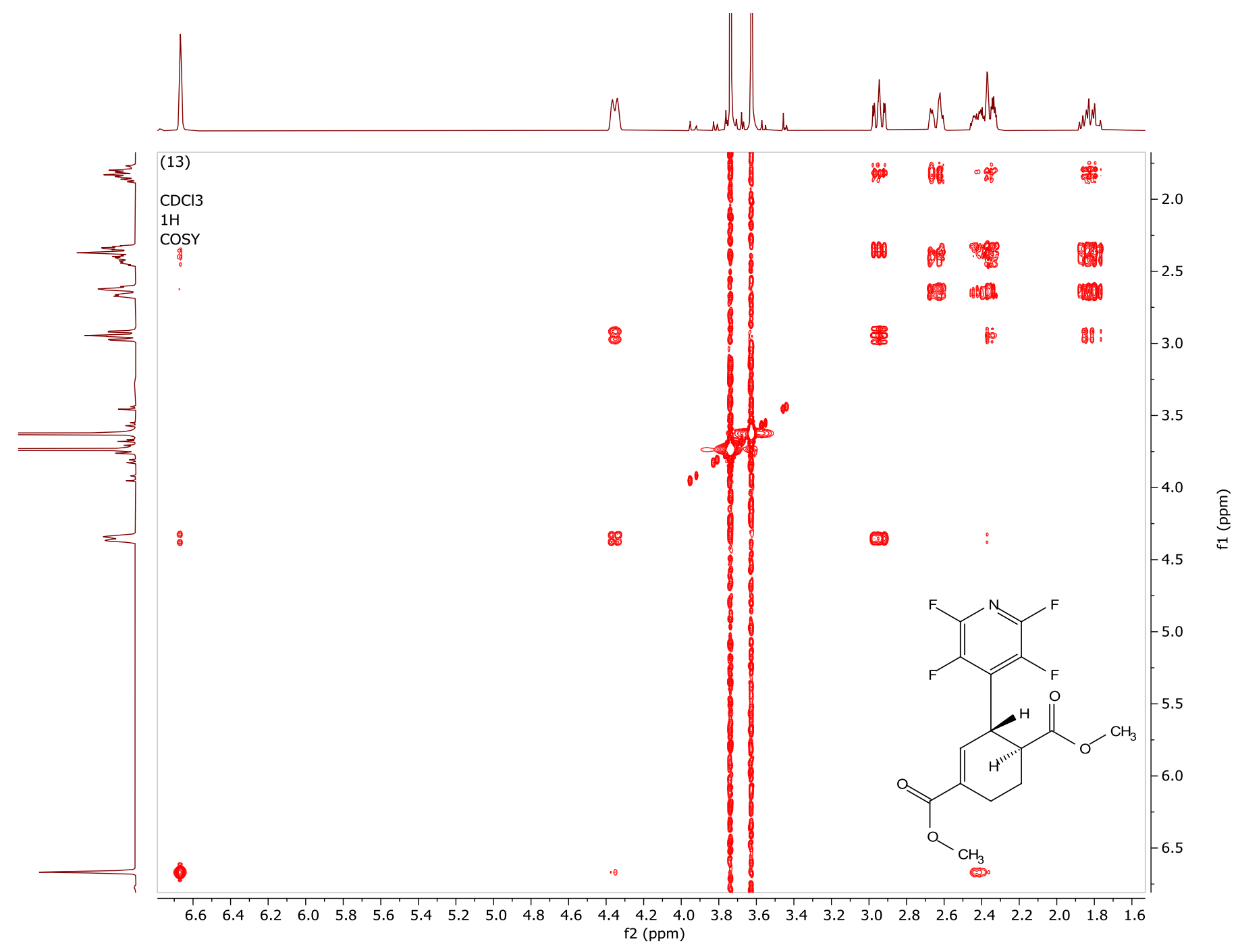


(14) Methyl 2',3',5',6'-tetrafluoro-5-methyl-4'-pentanoyl-1,4-dihydro-[1,1'-biphenyl]-2- carboxylate

${ }^{19} \mathbf{F}$ NMR $\left(\mathrm{CDCl}_{3}, 376 \mathrm{MHz}\right)$

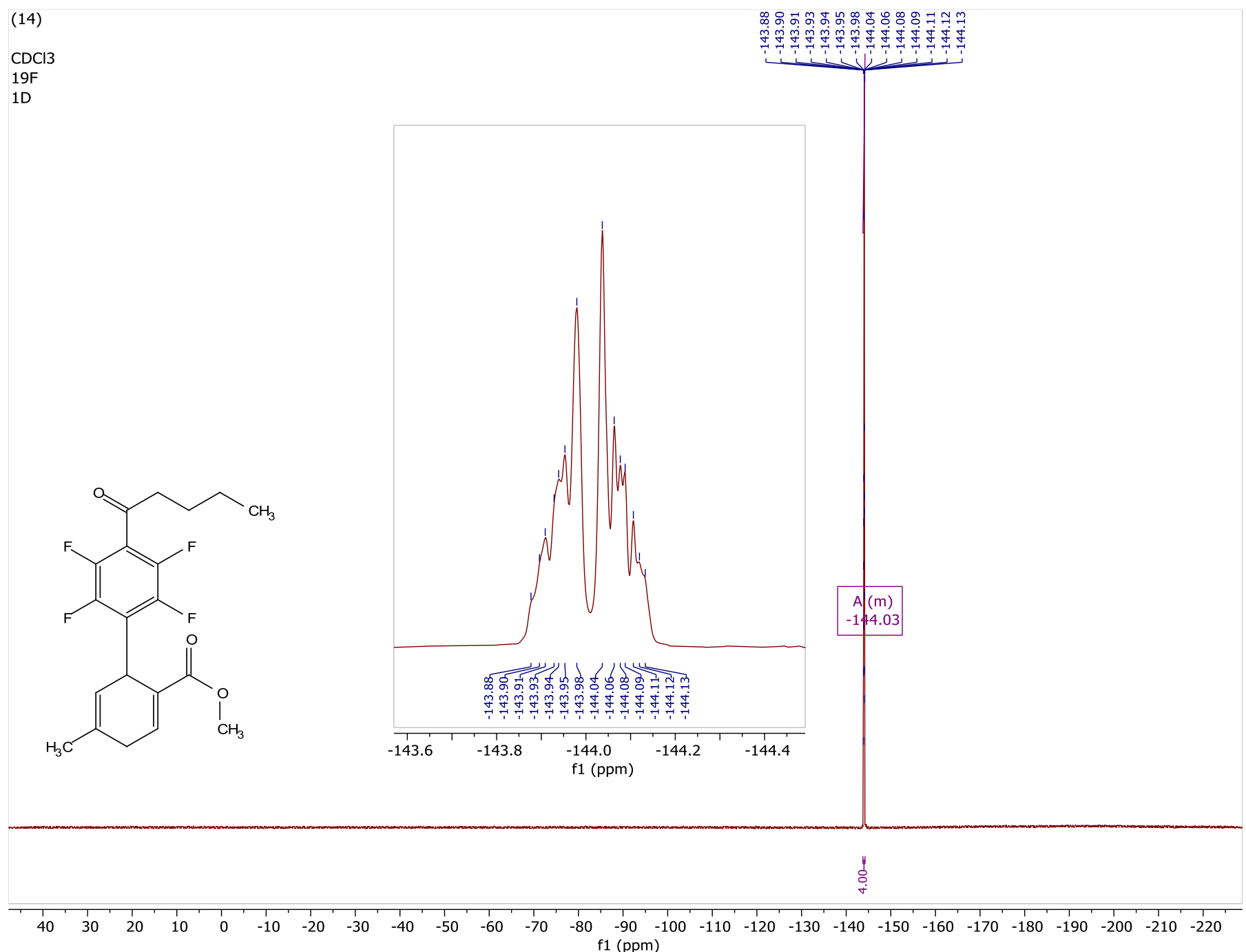


${ }^{1} \mathbf{H}$ NMR $\left(\mathrm{CDCl}_{3}, 400 \mathrm{MHz}\right)$

(14)

$\mathrm{CDCl} 3$

$1 \mathrm{H}$

$1 \mathrm{D}$

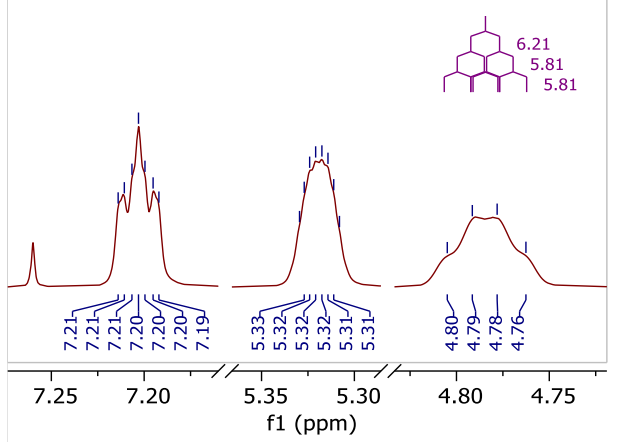

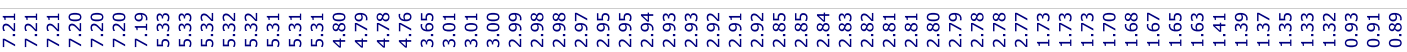
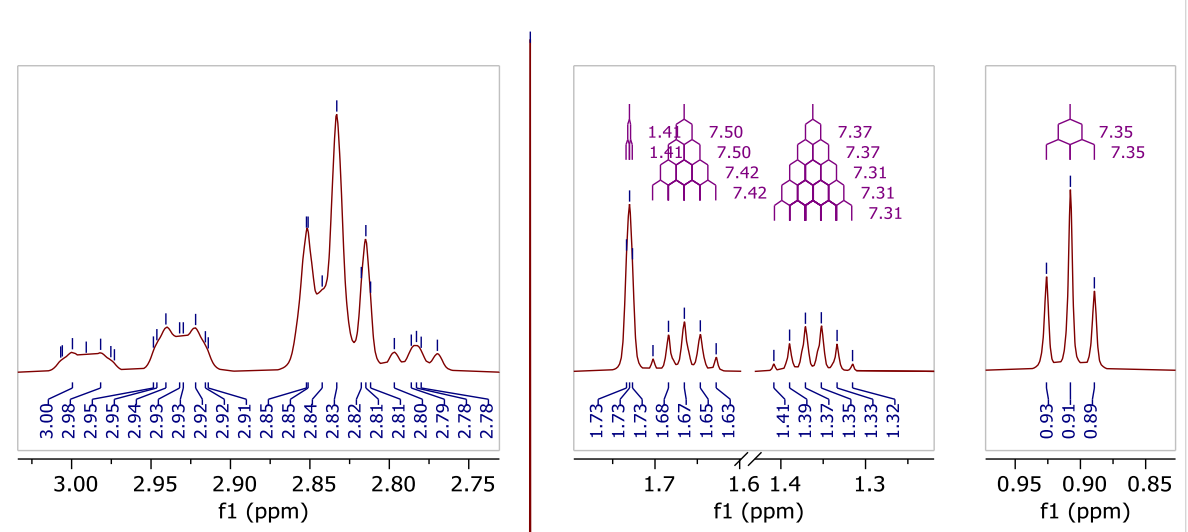
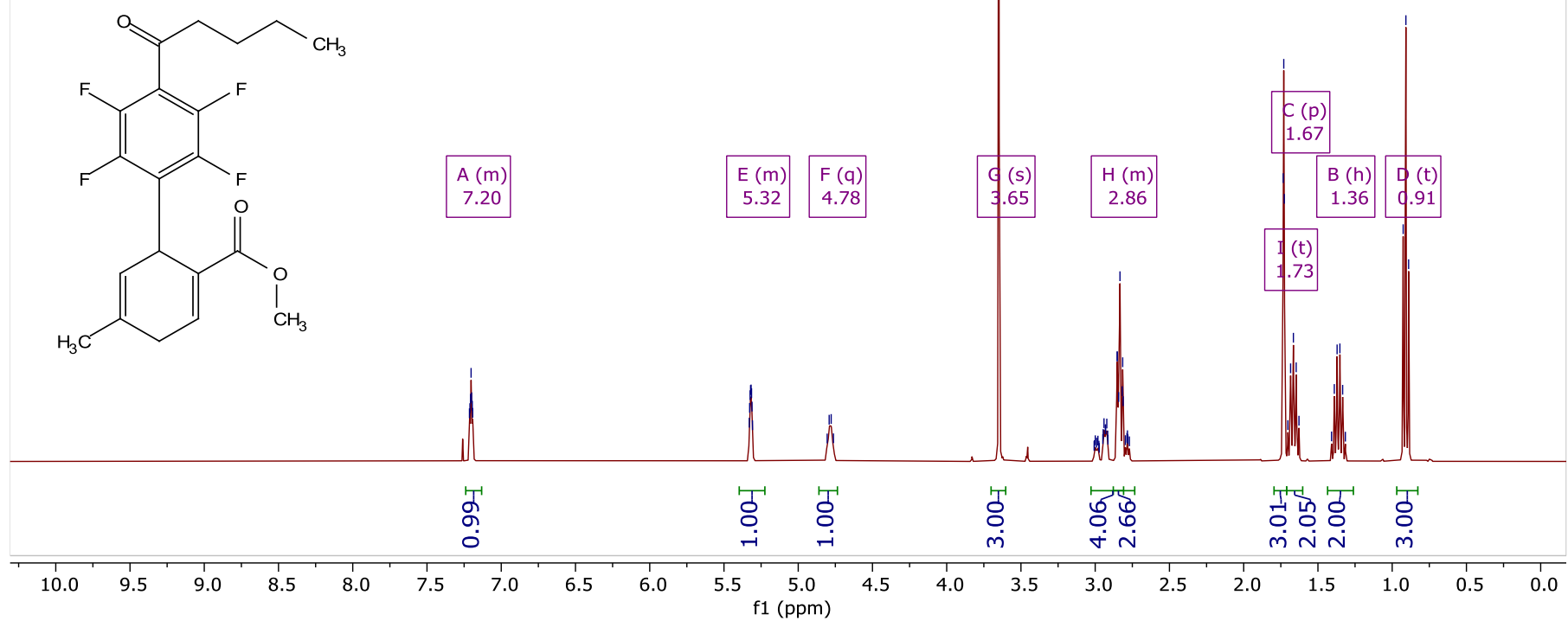
${ }^{13} \mathbf{C}\left\{{ }^{1} \mathrm{H}\right\}$ NMR $\left(\mathrm{CDCl}_{3}, 101 \mathrm{MHz}\right)$

(14)

$\mathrm{CDCl} 3$
$13 \mathrm{C}$

$13 \mathrm{C}$
$1 \mathrm{D}$

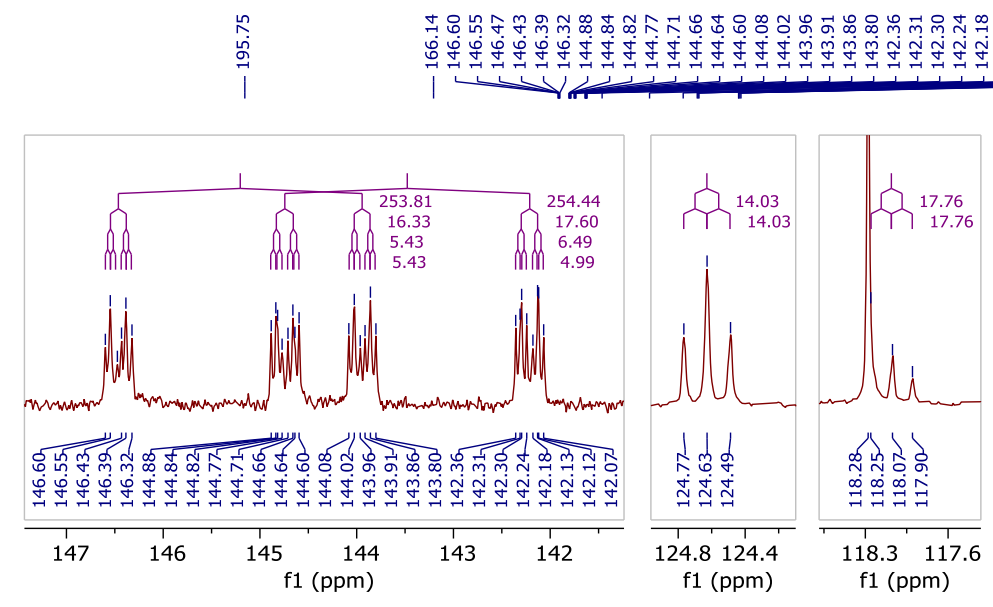

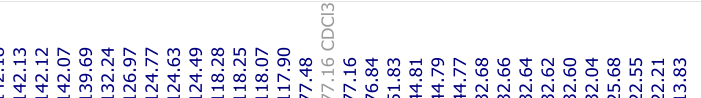

1.93

$\operatorname{sim} 1.930$

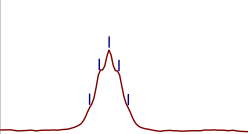

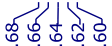
लंल लं लं ले

\begin{tabular}{l|l|l}
32.8 & 32.6 & 32.4
\end{tabular}

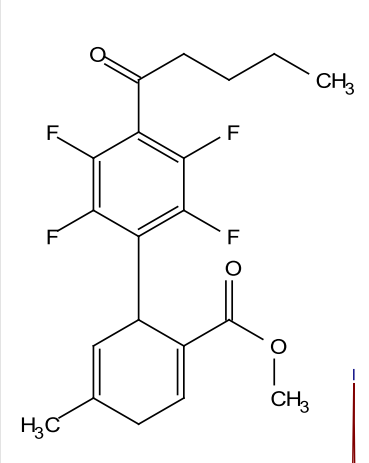

|llld

$\begin{array}{lllllllllllllllll}150 & 240 & 230 & 220 & 210 & 200 & 190 & 180 & 170 & 160 & 150 & 140 & 130 & 120 & 110 & 1\end{array}$ f1 (ppm) 
${ }^{1} \mathrm{H}-{ }^{13} \mathrm{C}$ HSQC

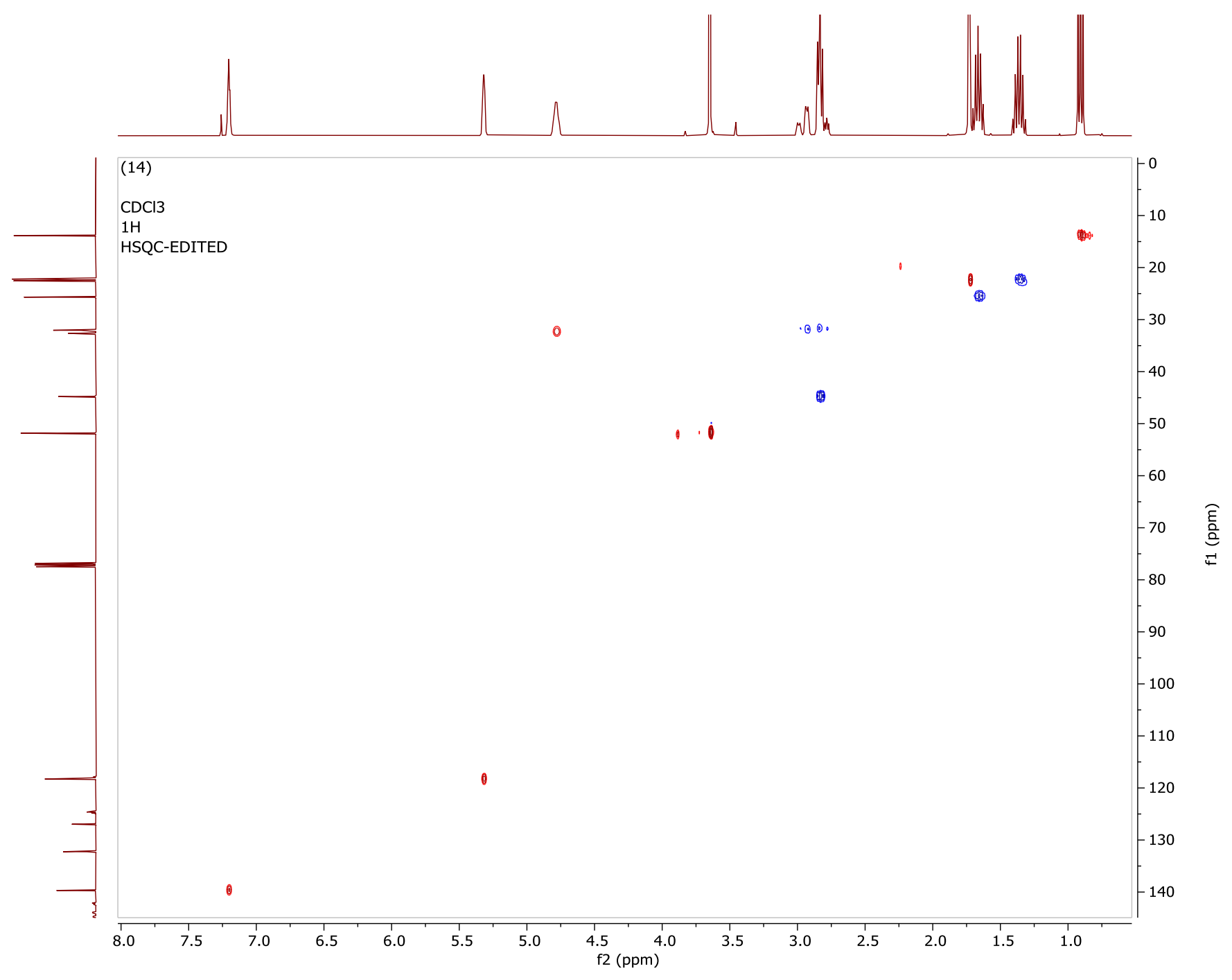


(15) dimethyl 2',3',5',6' '-tetrafluoro-5-methyl-1,4-dihydro-[1,1' -biphenyl]-2,4' -dicarboxylate

${ }^{19}$ F NMR $\left(\mathrm{CDCl}_{3}, 564 \mathrm{MHz}\right)$

(15)

$\mathrm{CDCl} 3$
$19 \mathrm{~F}$

$19 \mathrm{~F}$
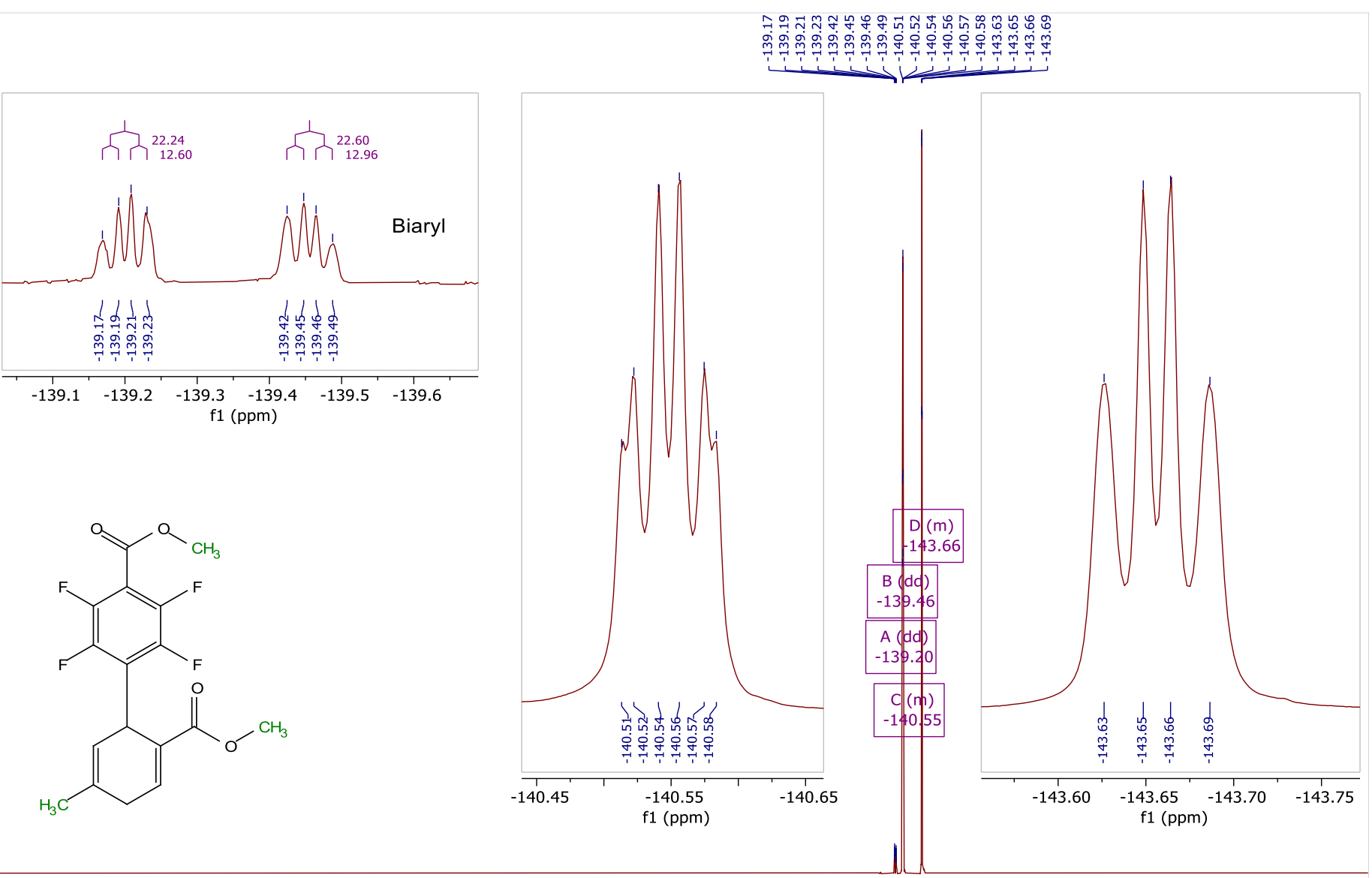

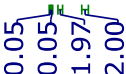

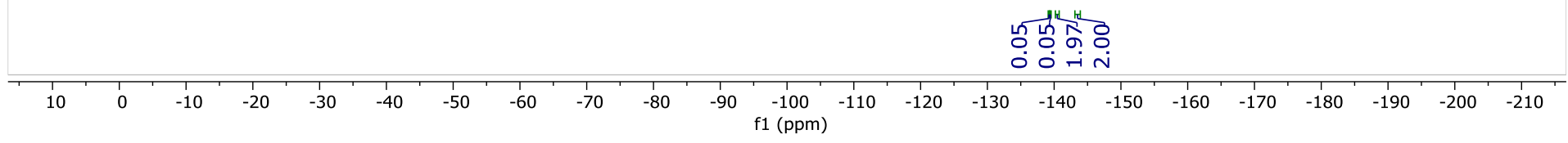


${ }^{1} \mathbf{H}$ NMR $\left(\mathrm{CDCl}_{3}, 599 \mathrm{MHz}\right)$

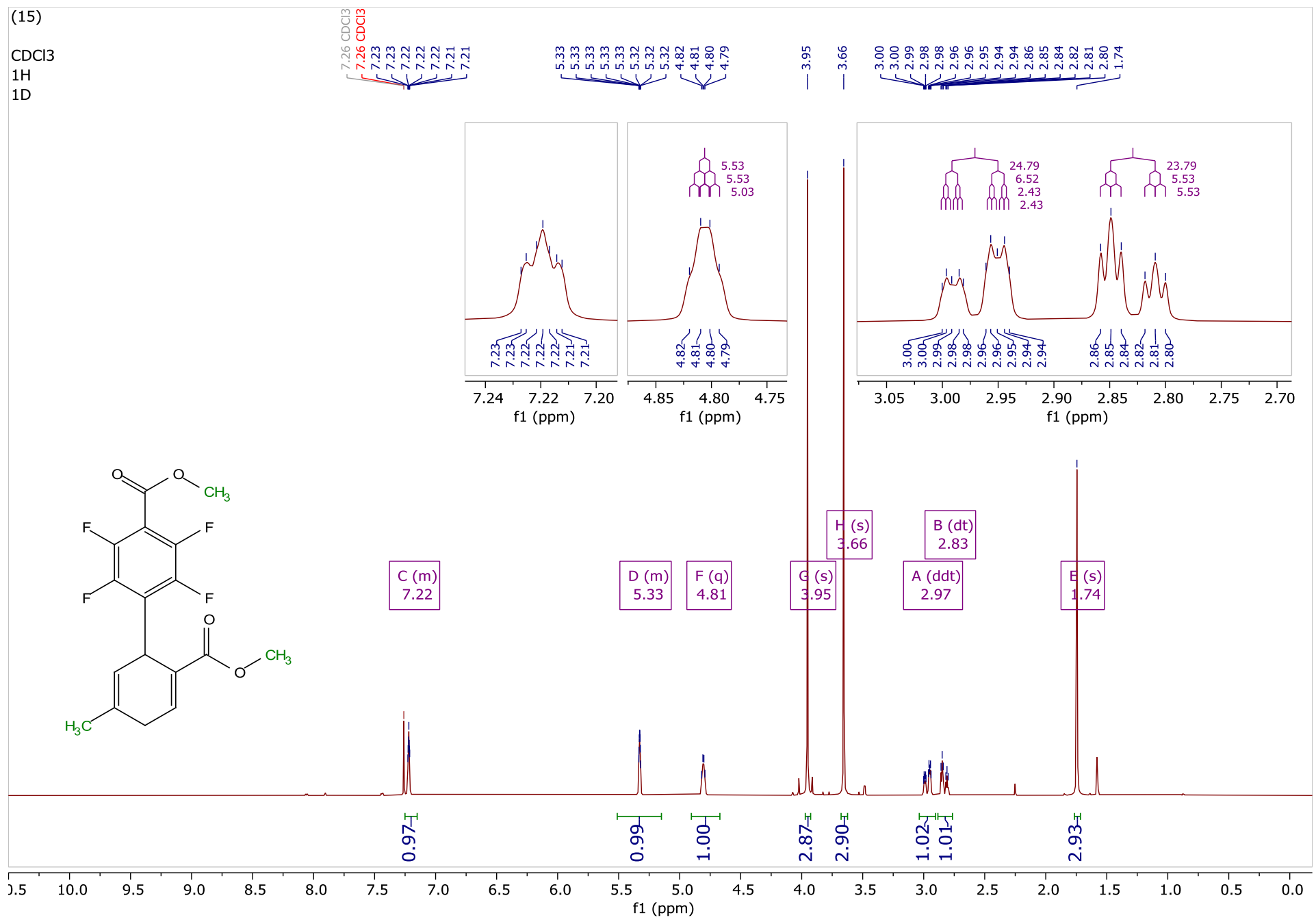


${ }^{13} \mathbf{C}\left\{{ }^{1} \mathrm{H}\right\}$ NMR $\left(\mathrm{CDCl}_{3}, 151 \mathrm{MHz}\right)$

(15)

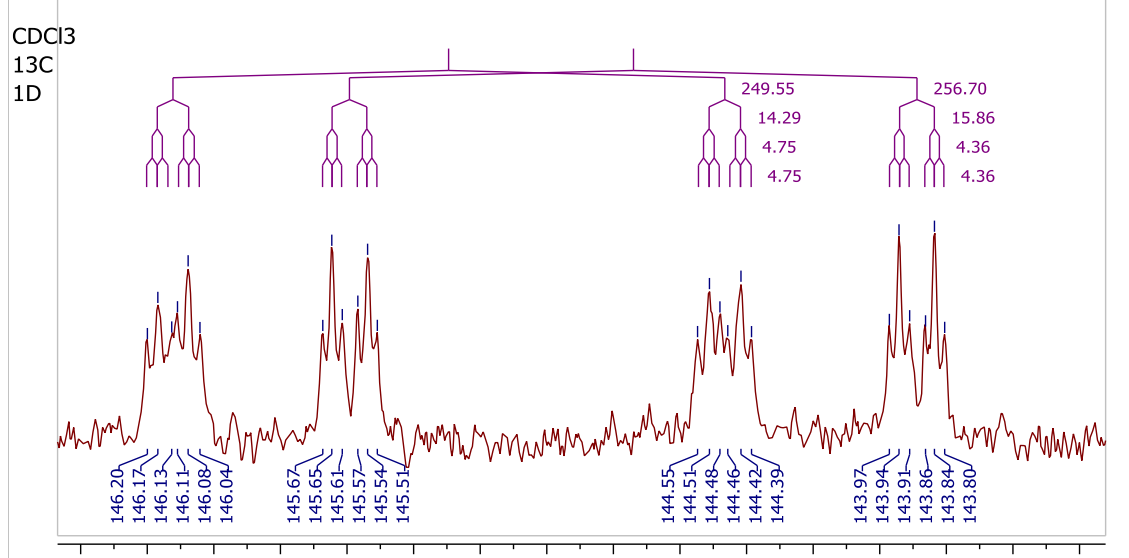

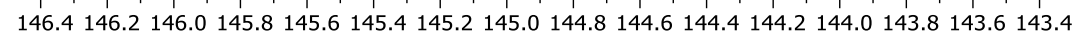
f1 $(\mathrm{ppm})$
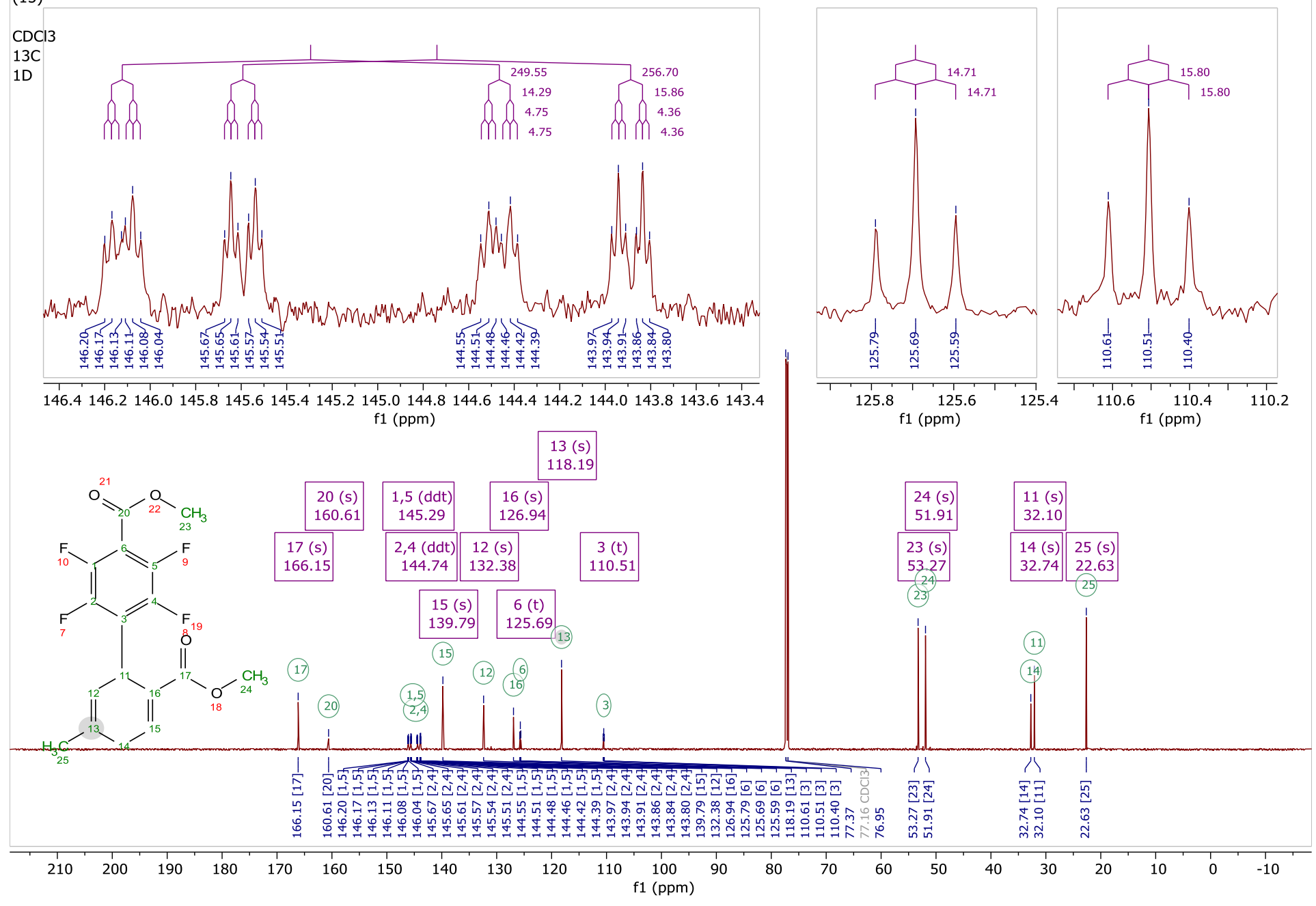
(16) methyl 4-methyl-6-(perfluoropyridin-4-yl)cyclohexa-1,4-diene-1-carboxylate

(16) UV trace for column chromatography

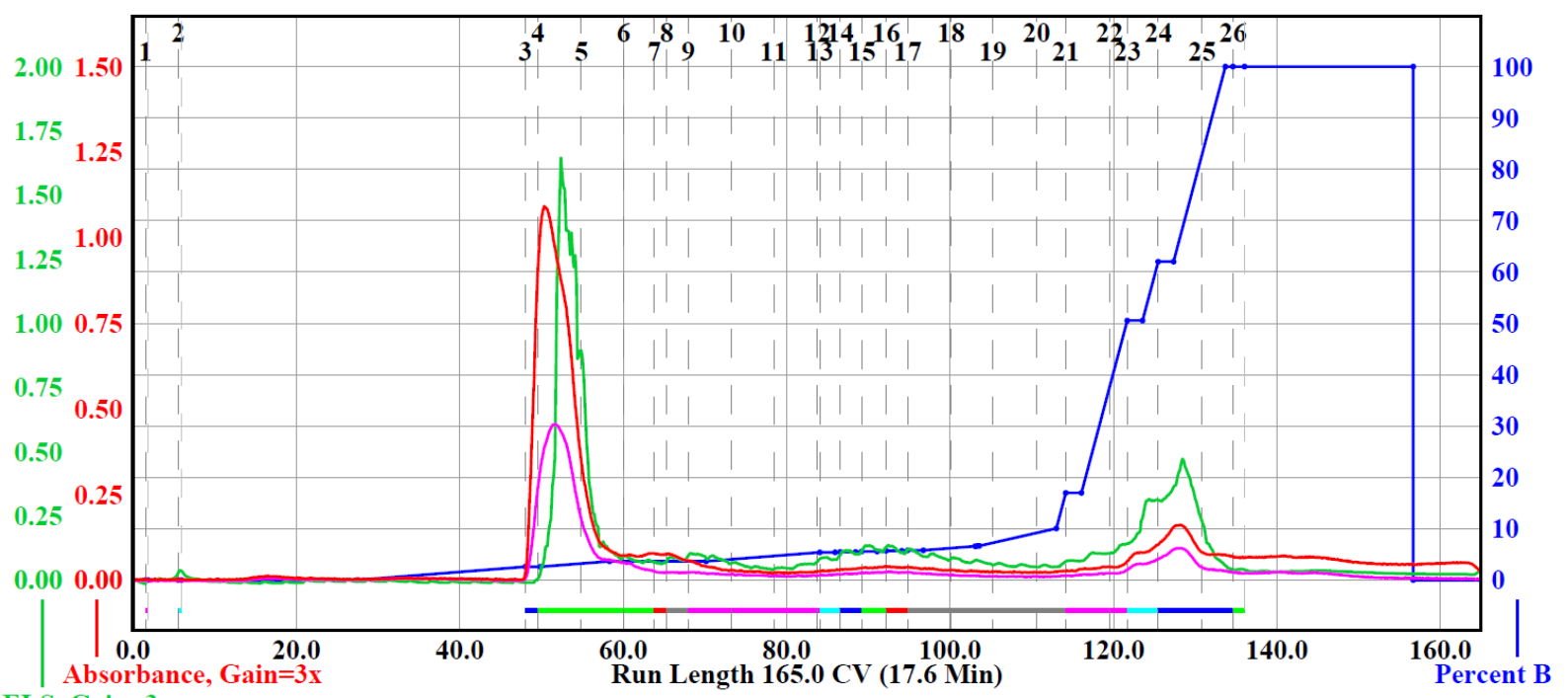
ELS, Gain $=3 \mathrm{x}$ 
${ }^{19}$ F NMR $\left(\mathrm{CD}_{3} \mathrm{CN}, 564 \mathrm{MHz}\right)$

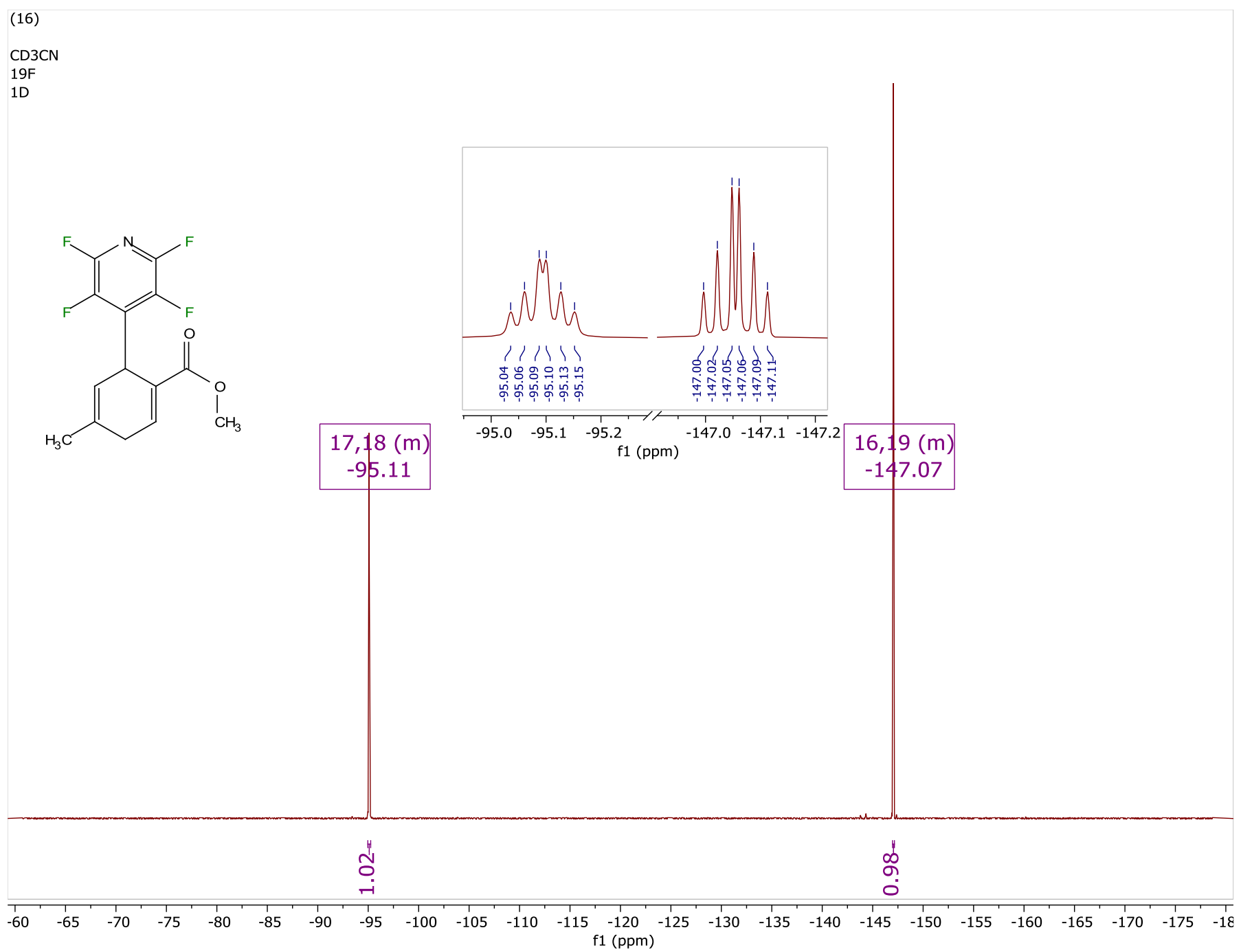


${ }^{1} \mathbf{H}$ NMR $\left(\mathrm{CD}_{3} \mathrm{CN}, 599 \mathrm{MHz}\right)$

(16)

$\mathrm{CD} 3 \mathrm{CN}$
$1 \mathrm{H}$
$1 \mathrm{D}$
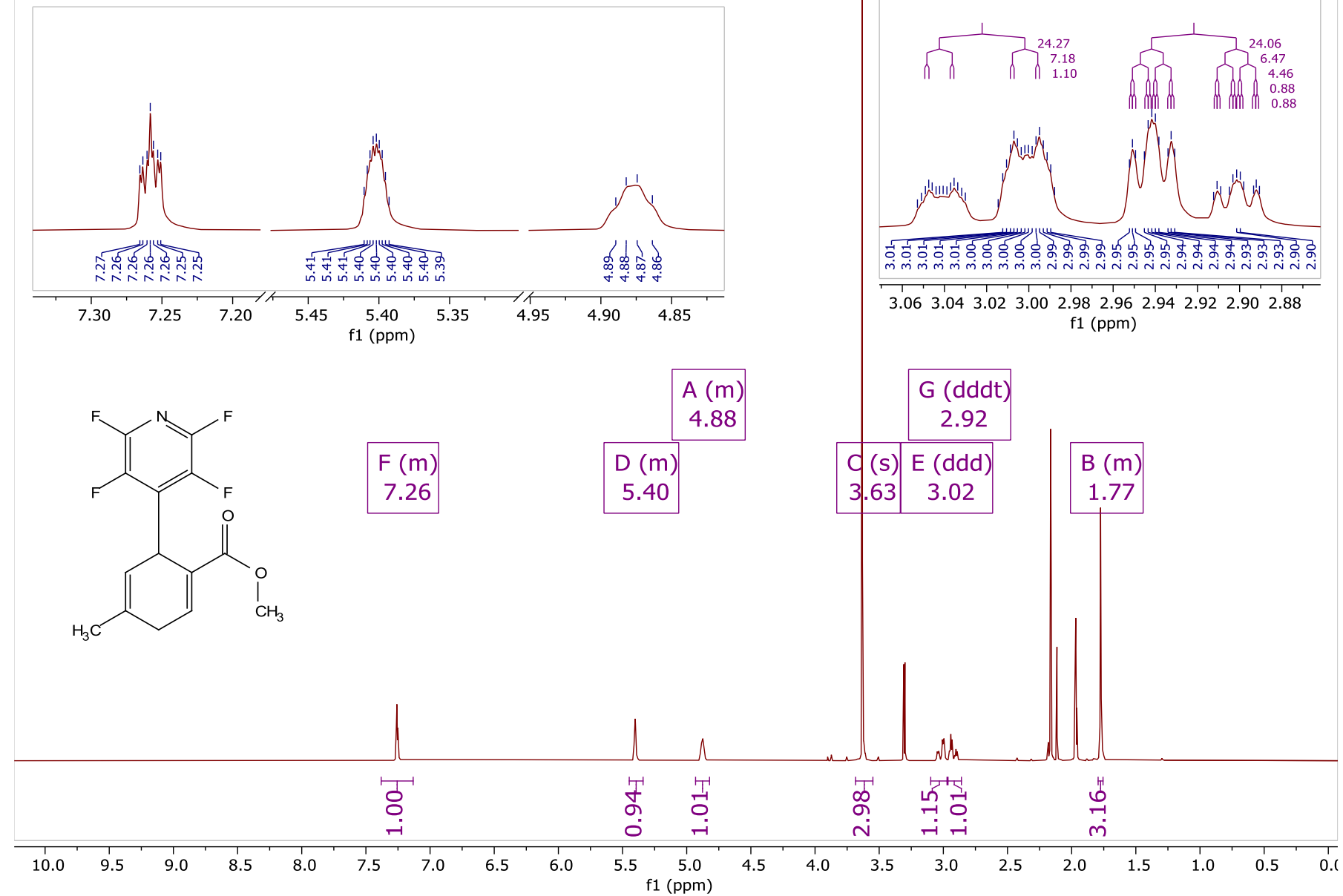
${ }^{13} \mathbf{C}\left\{{ }^{1} \mathrm{H}\right\}$ NMR $\left(\mathrm{CD}_{3} \mathrm{CN}, 151 \mathrm{MHz}\right)$

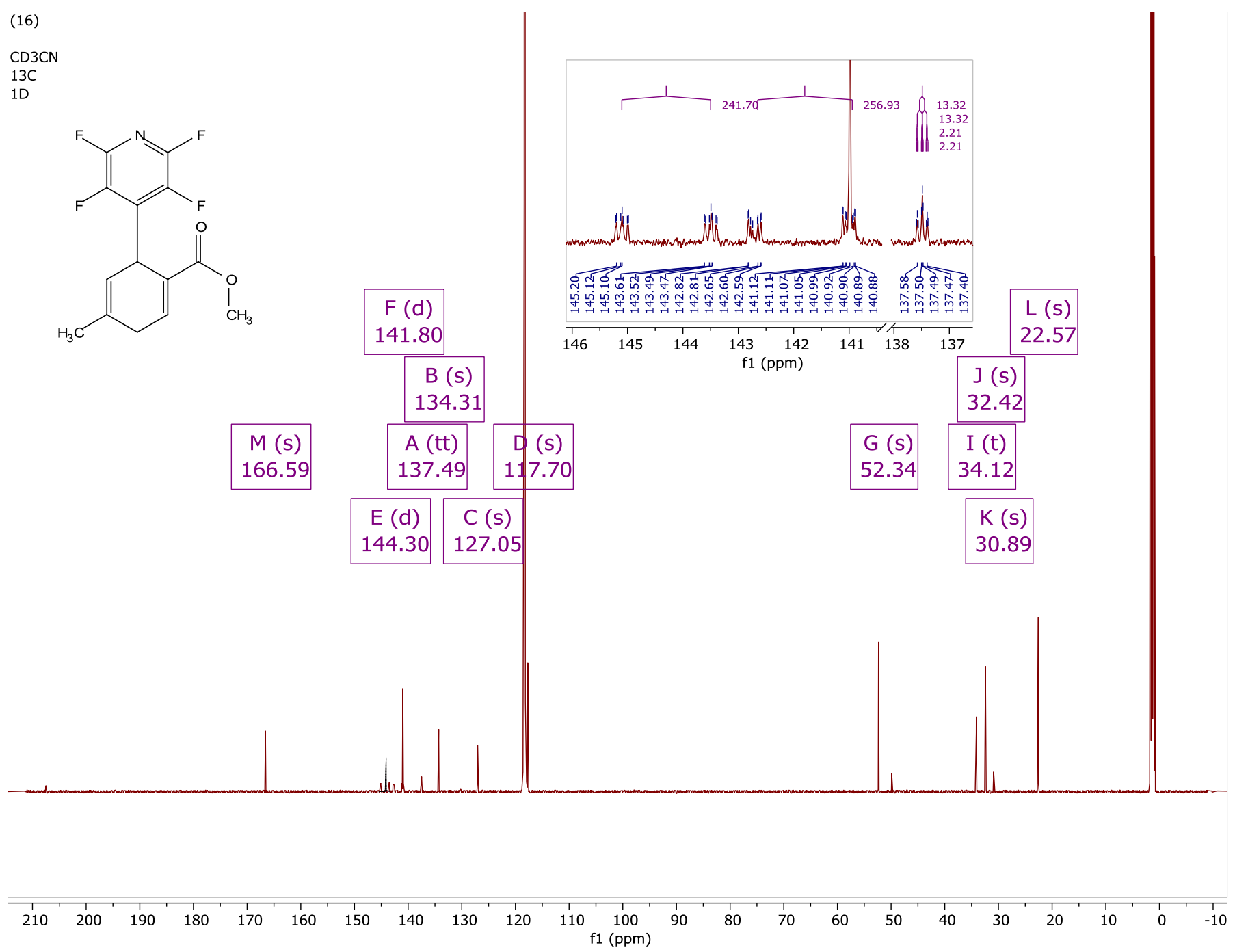


${ }^{1} \mathrm{H}-{ }^{19} \mathrm{~F}$ HOESY

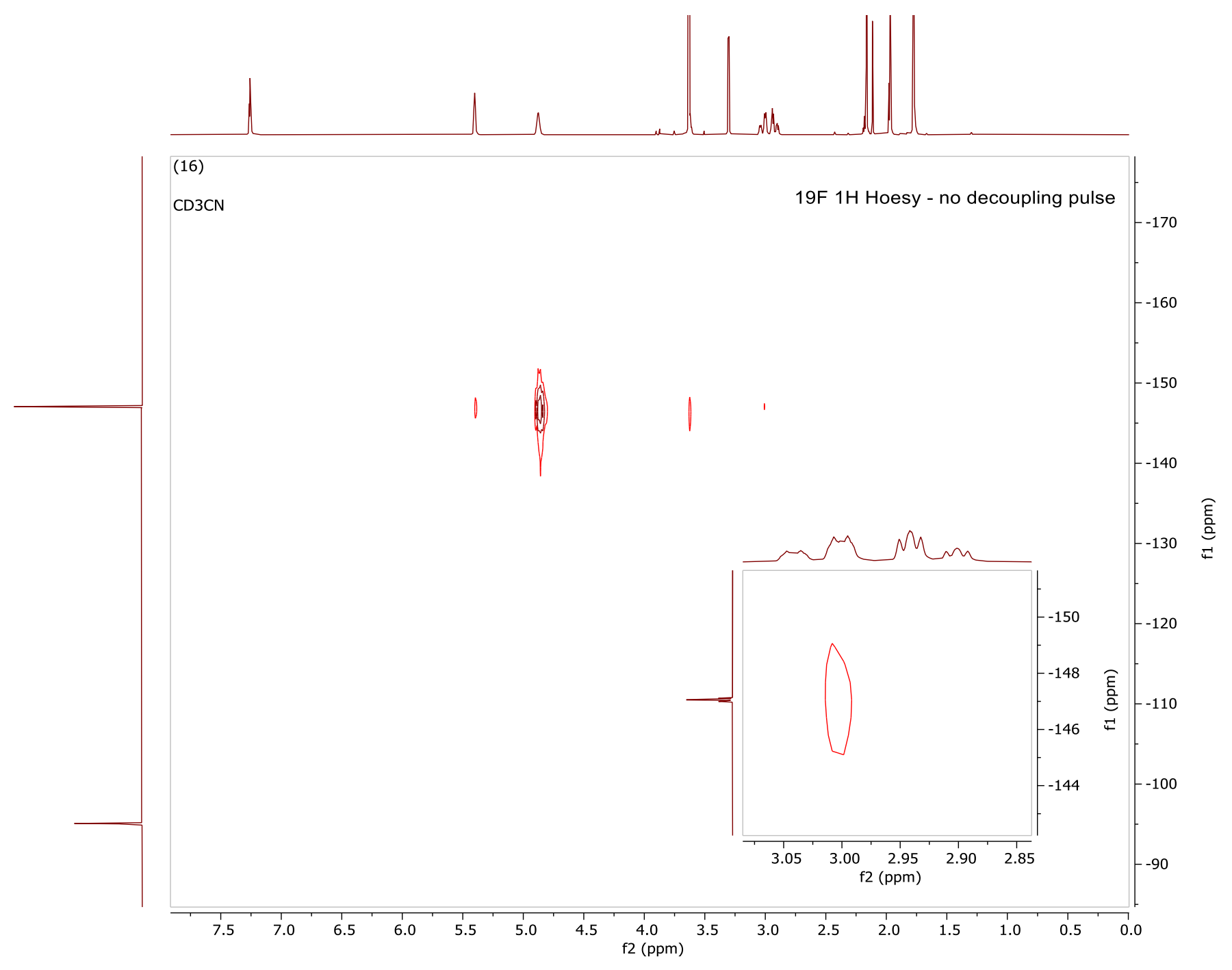


(17) methyl 4-(tert-butyl)-6-(perfluoropyridin-4-yl)cyclohexa-1,4-diene-1-carboxylate

${ }^{19}$ F NMR $\left(\mathrm{CDCl}_{3}, 376 \mathrm{MHz}\right)$

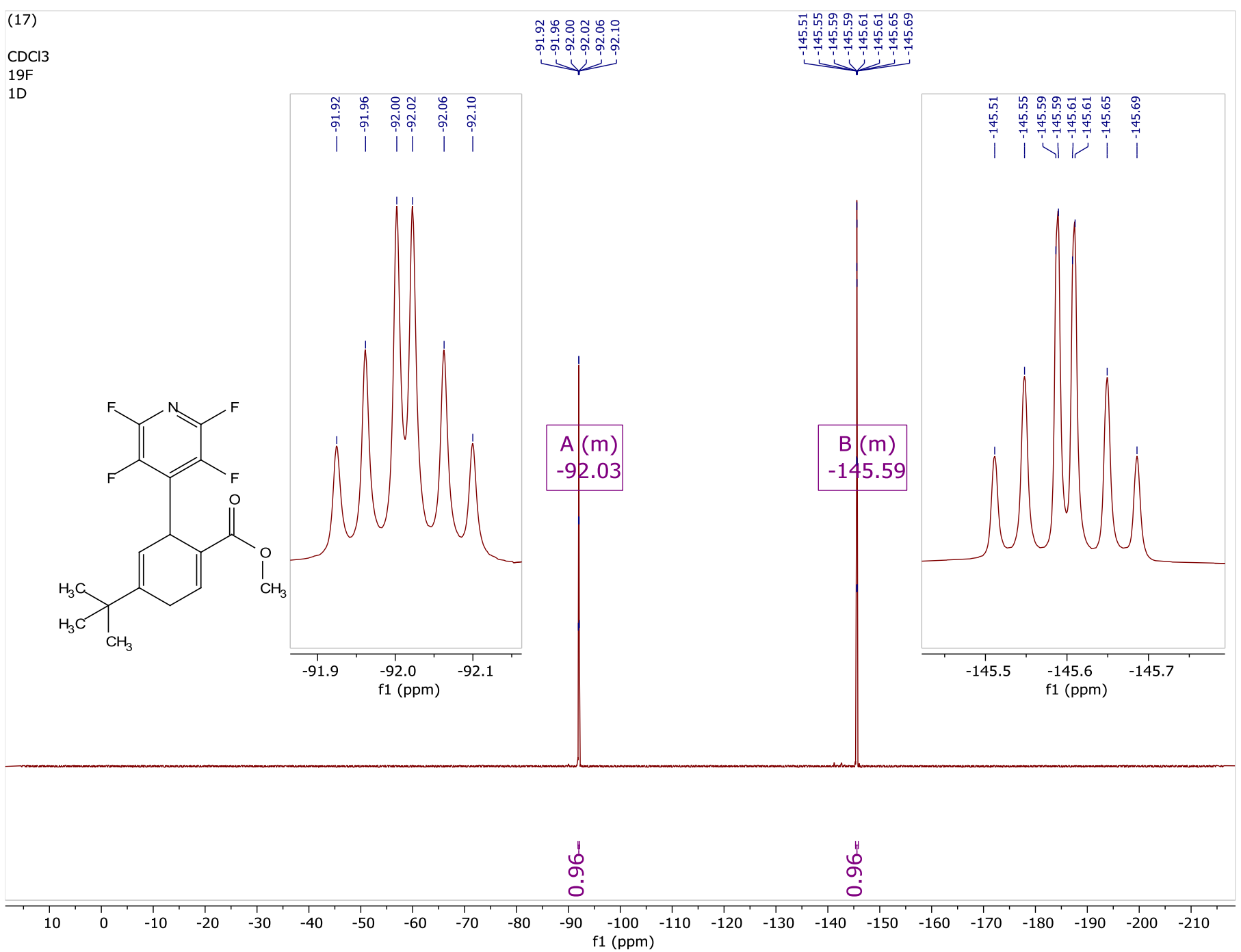


${ }^{\mathbf{1}} \mathbf{H} \mathbf{N M R}\left(\mathrm{CDCl}_{3}, 400 \mathrm{MHz}\right)$

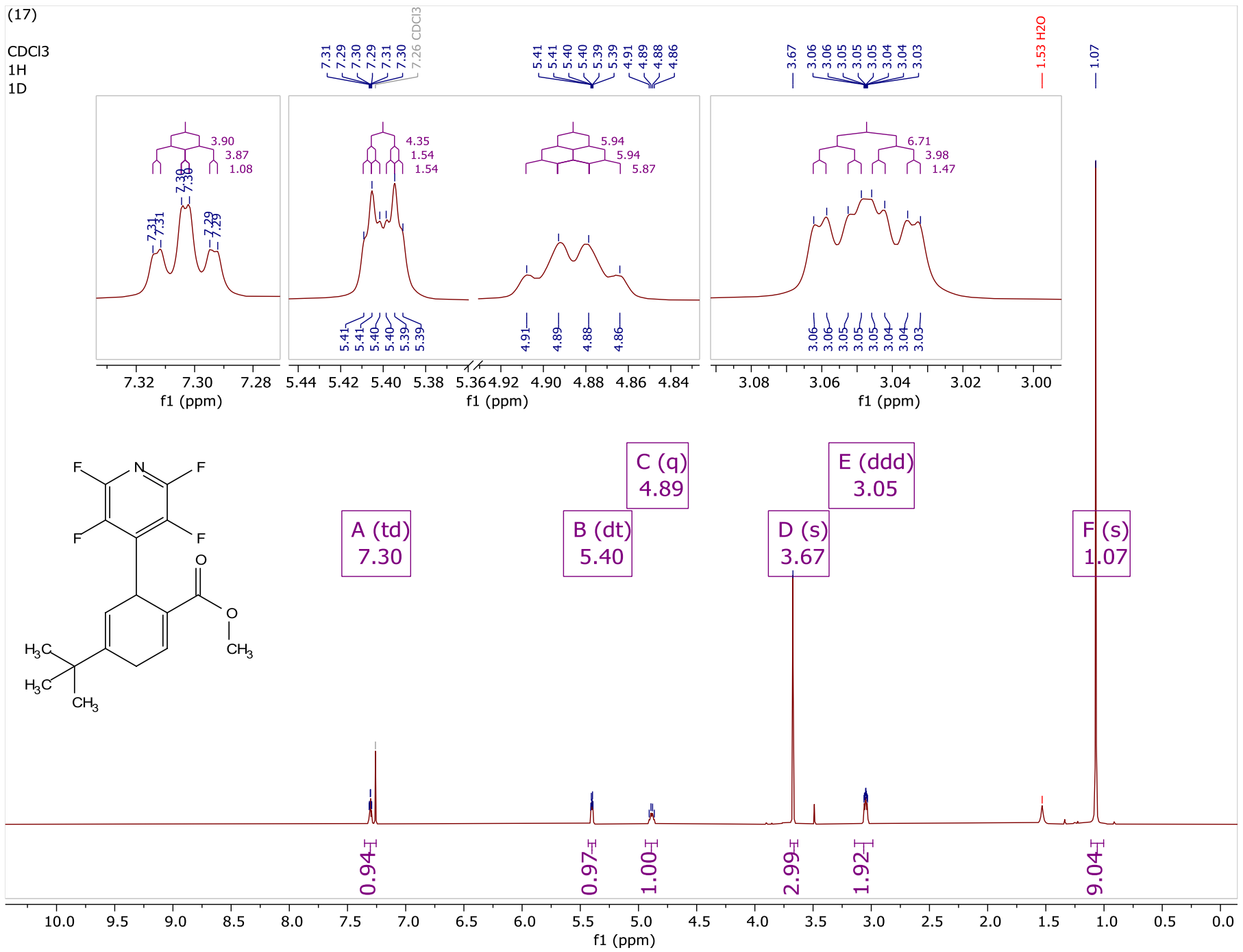




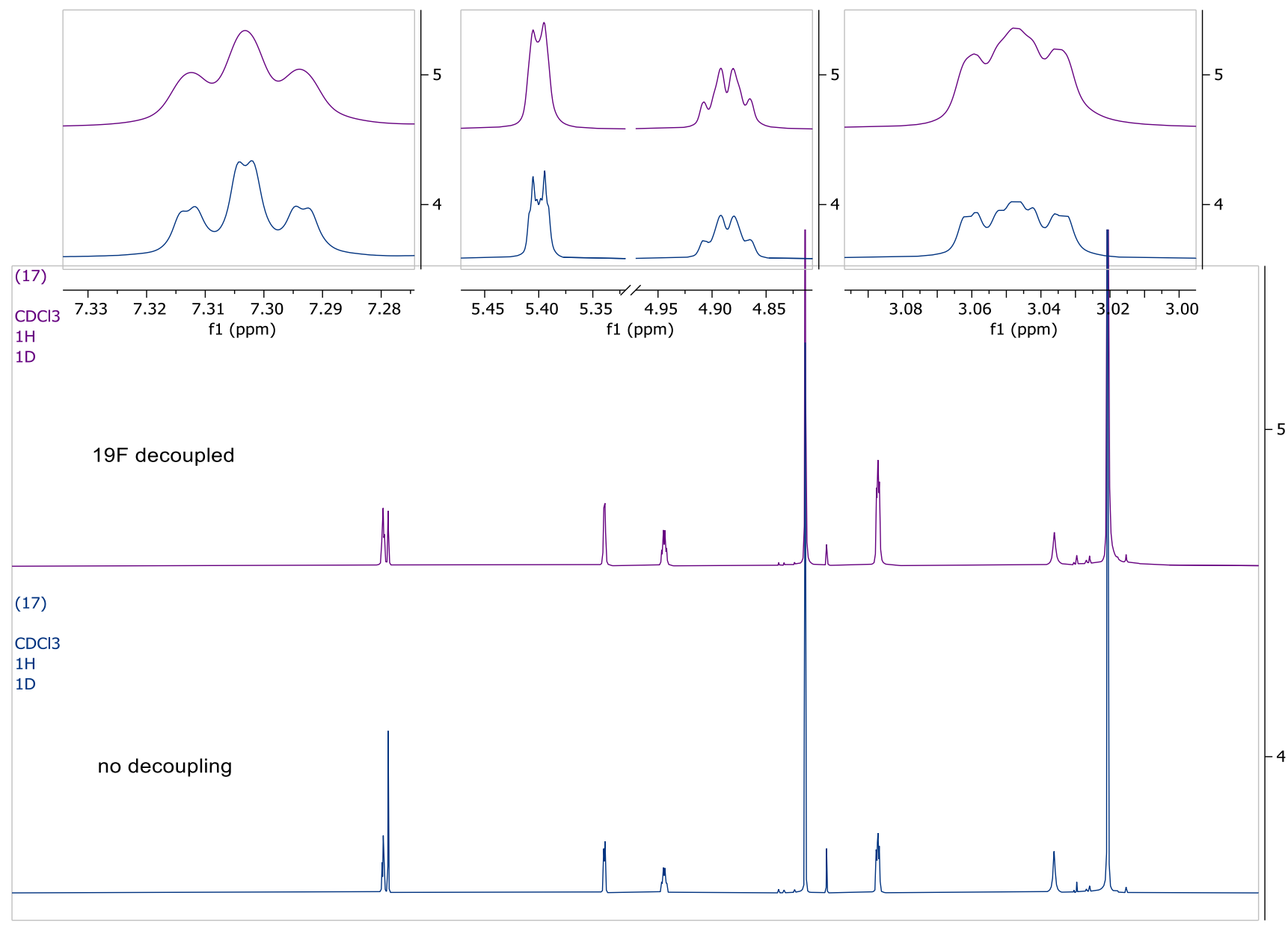

$\begin{array}{llllllllllllllllllllll}10.0 & 9.5 & 9.0 & 8.5 & 8.0 & 7.5 & 7.0 & 6.5 & 6.0 & 5.5 & 5.0 & 4.5 & 4.0 & 3.5 & 3.0 & 2.5 & 2.0 & 1.5 & 1.0 & 0.5 & 0.0\end{array}$ 
${ }^{13} \mathrm{C}\left\{{ }^{1} \mathrm{H}\right\}$ NMR $\left(\mathrm{CDCl}_{3}, 101 \mathrm{MHz}\right)$

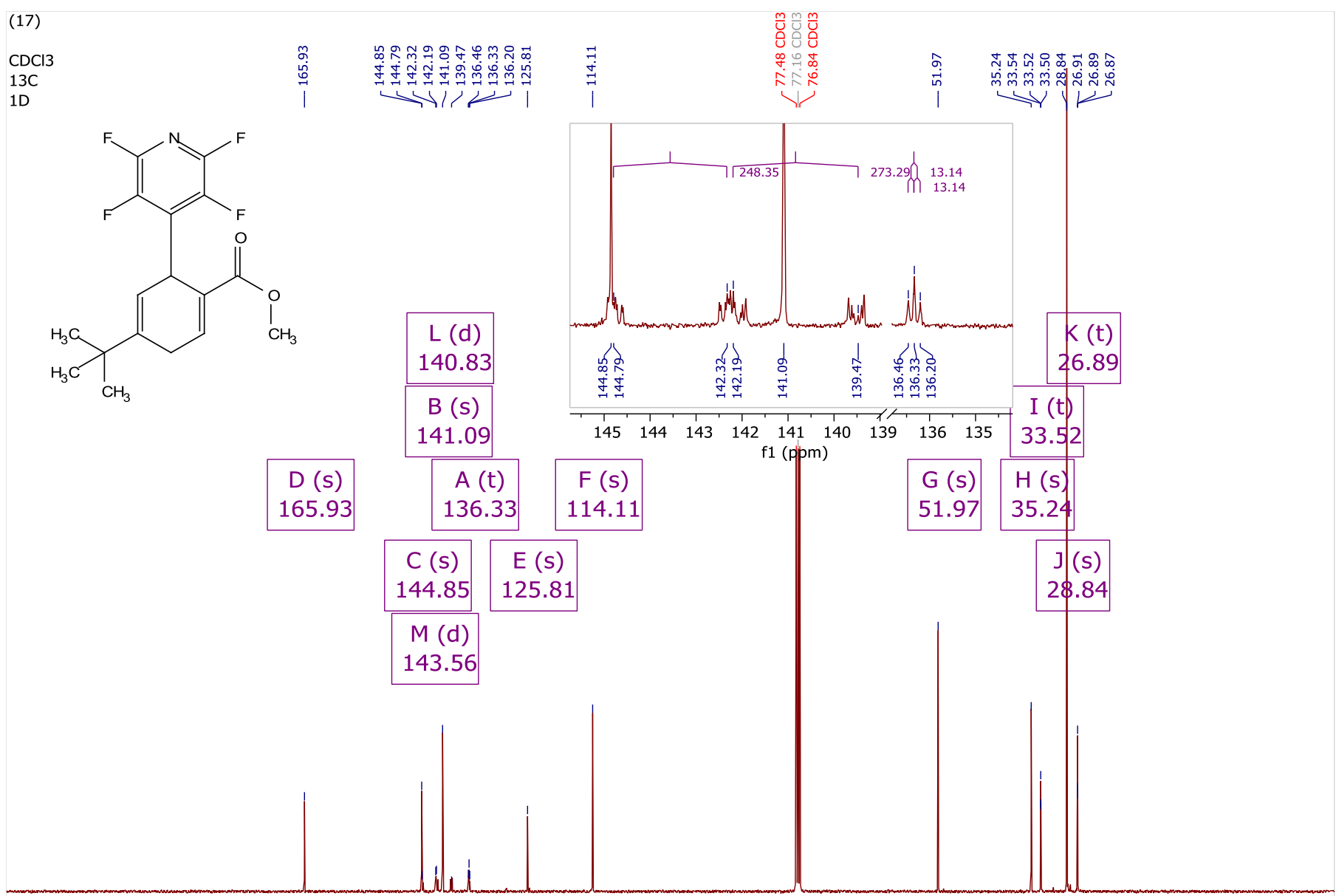

$\begin{array}{lllllllllllllllllllllllllll}210 & 200 & 190 & 180 & 170 & 160 & 150 & 140 & 130 & 120 & 110 & 100 & 90 & 80 & 70 & 60 & 50 & 40 & 30 & 20 & 10 & 0 & -10\end{array}$ 
(18) methyl 2',3',5',6' -tetrafluoro-5-methyl-4' -(trifluoromethyl)-1,4-dihydro-[1,1'-biphenyl]-2-carboxylate

${ }^{19}$ F NMR $\left(\mathrm{CD}_{3} \mathrm{CN}, 564 \mathrm{MHz}\right)$

(18)

CD3CN

$19 F$

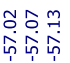

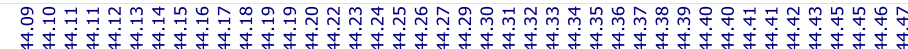
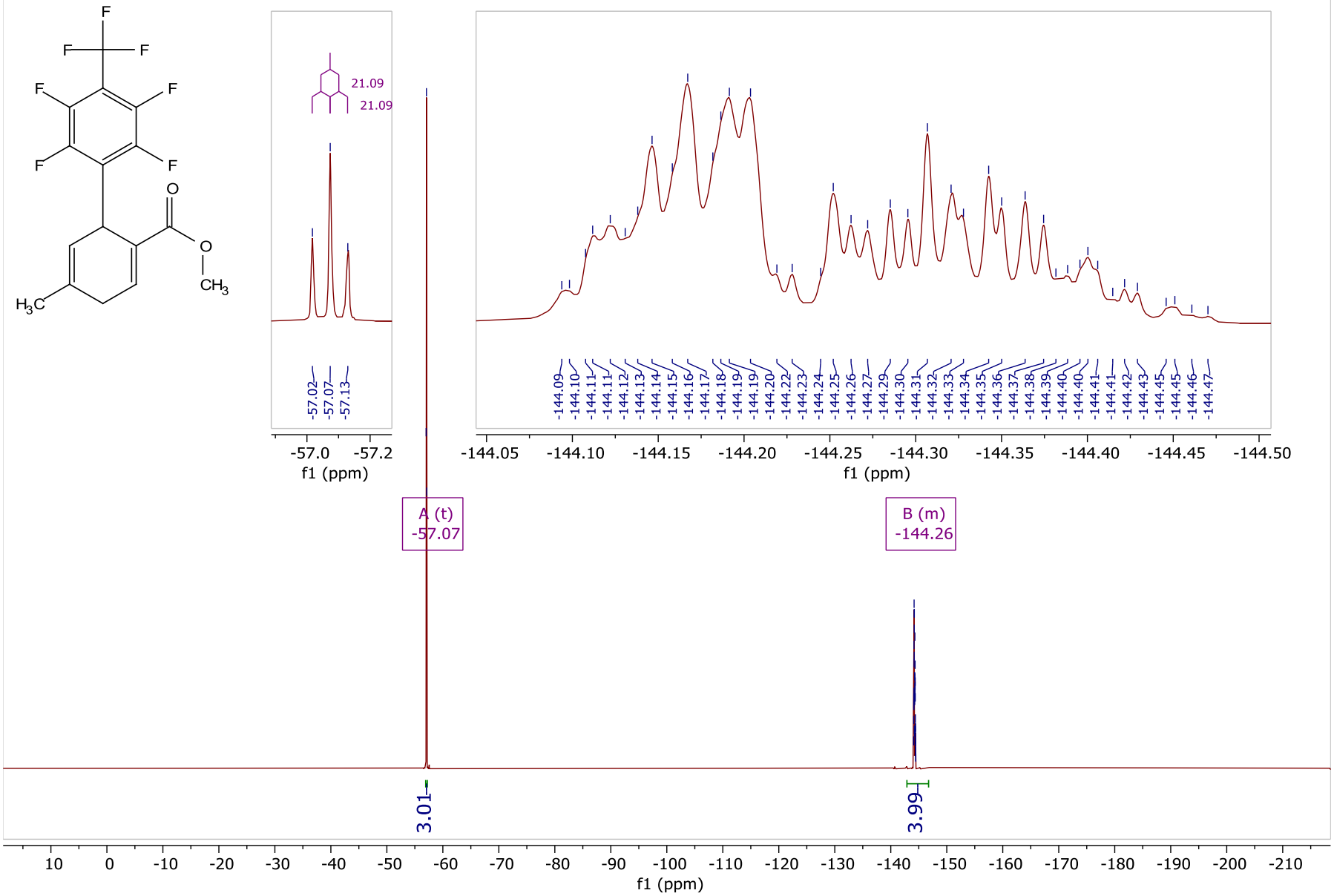
${ }^{1} \mathbf{H}$ NMR $\left(\mathrm{CD}_{3} \mathrm{CN}, 599 \mathrm{MHz}\right)$

(18)

CD3CN
$1 \mathrm{H}$
$1 \mathrm{D}$
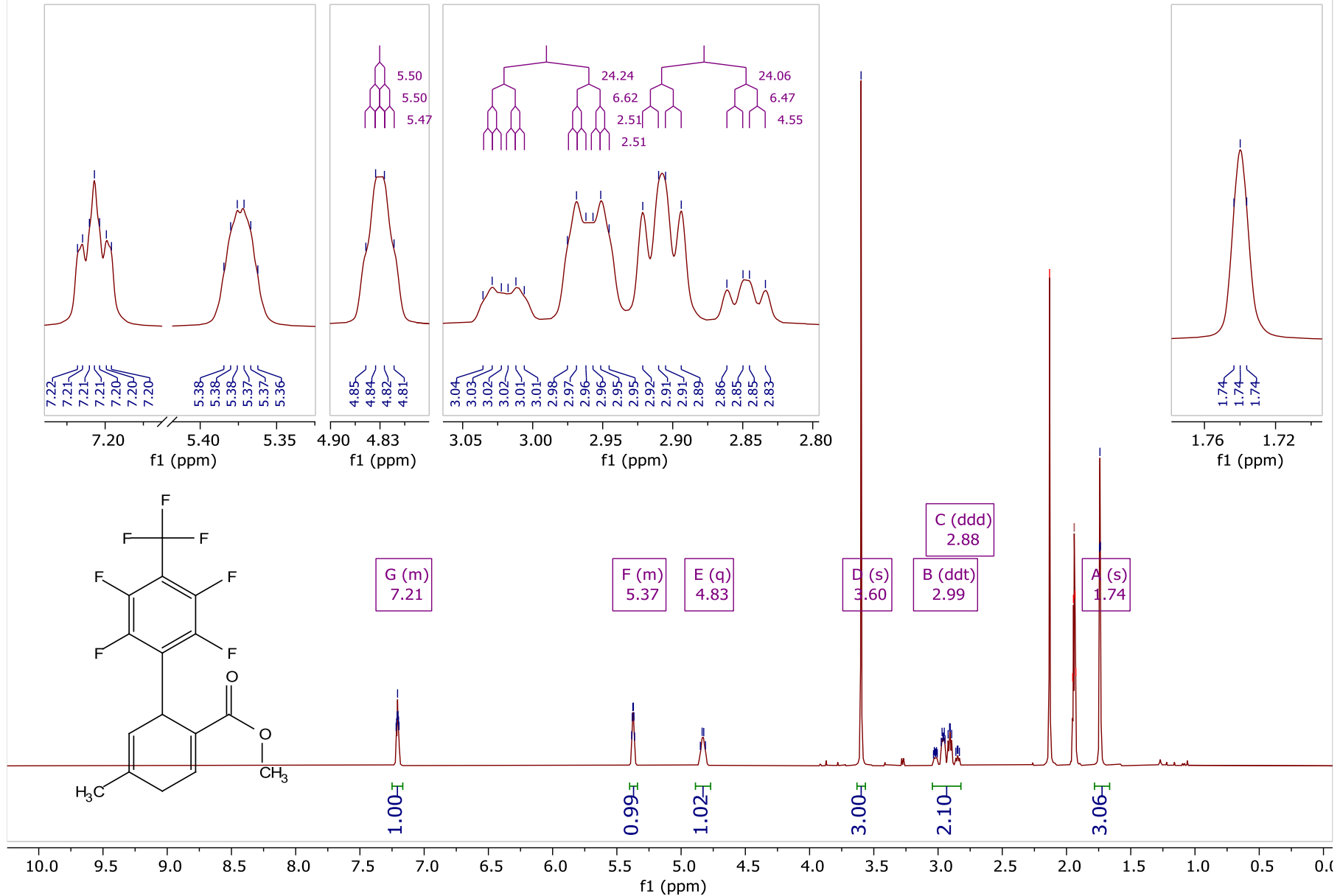

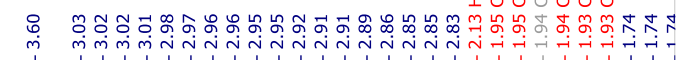

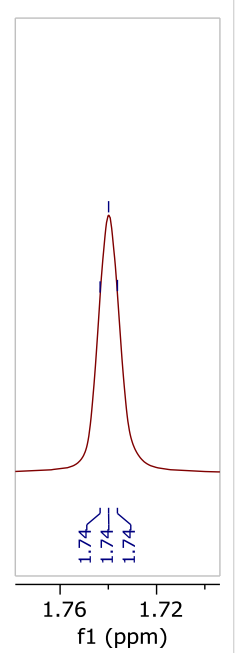


${ }^{13} \mathrm{C}\left\{{ }^{1} \mathrm{H}\right\}$ NMR $\left(\mathrm{CD}_{3} \mathrm{CN}, 151 \mathrm{MHz}\right)$

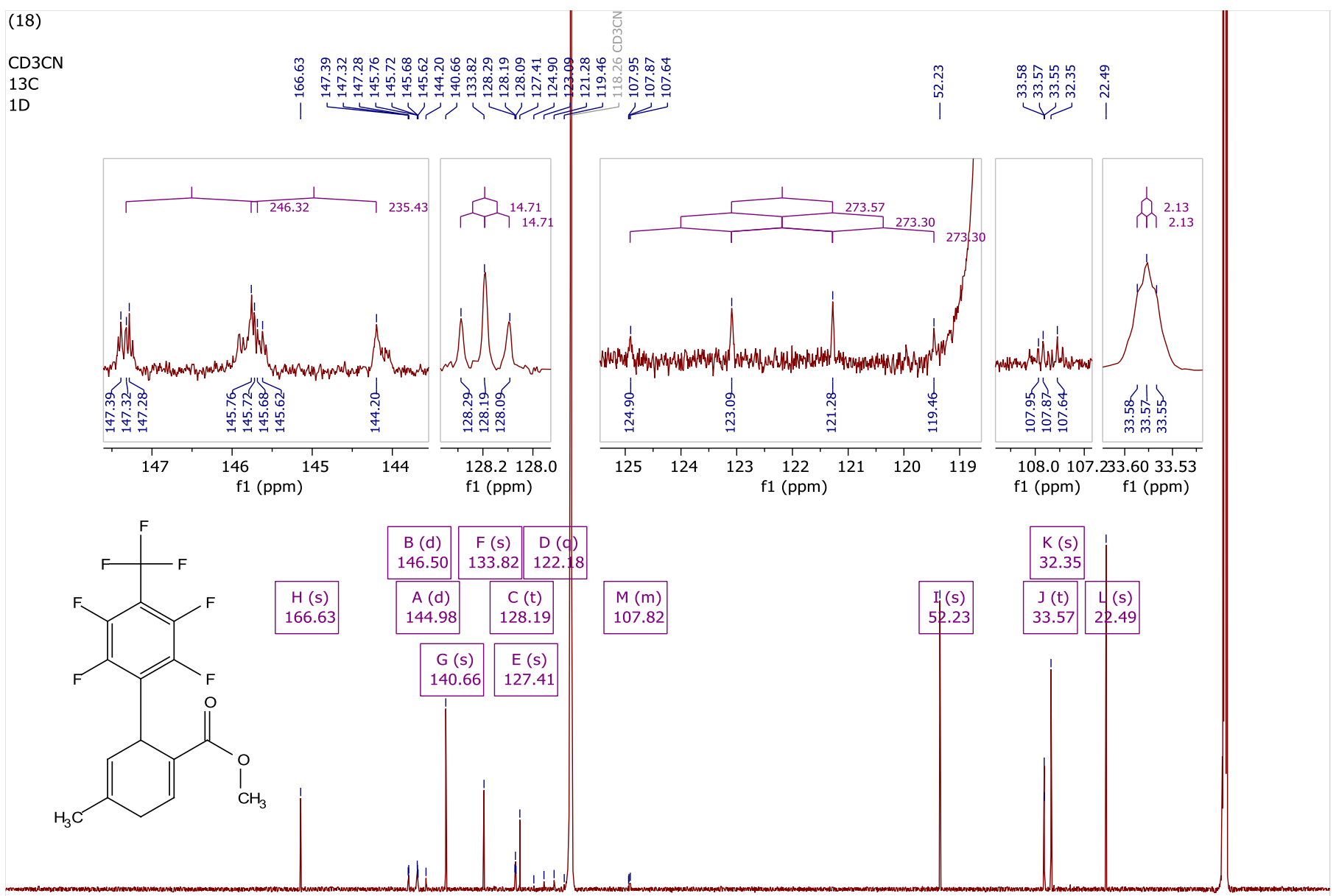

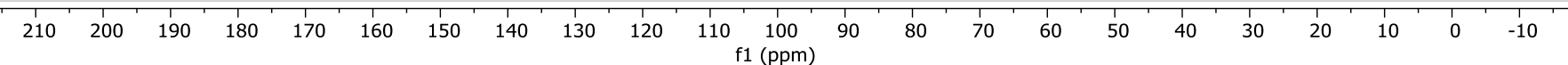


${ }^{19}$ F NMR $\left(\mathrm{CD}_{3} \mathrm{CN}, 376 \mathrm{MHz}\right)$

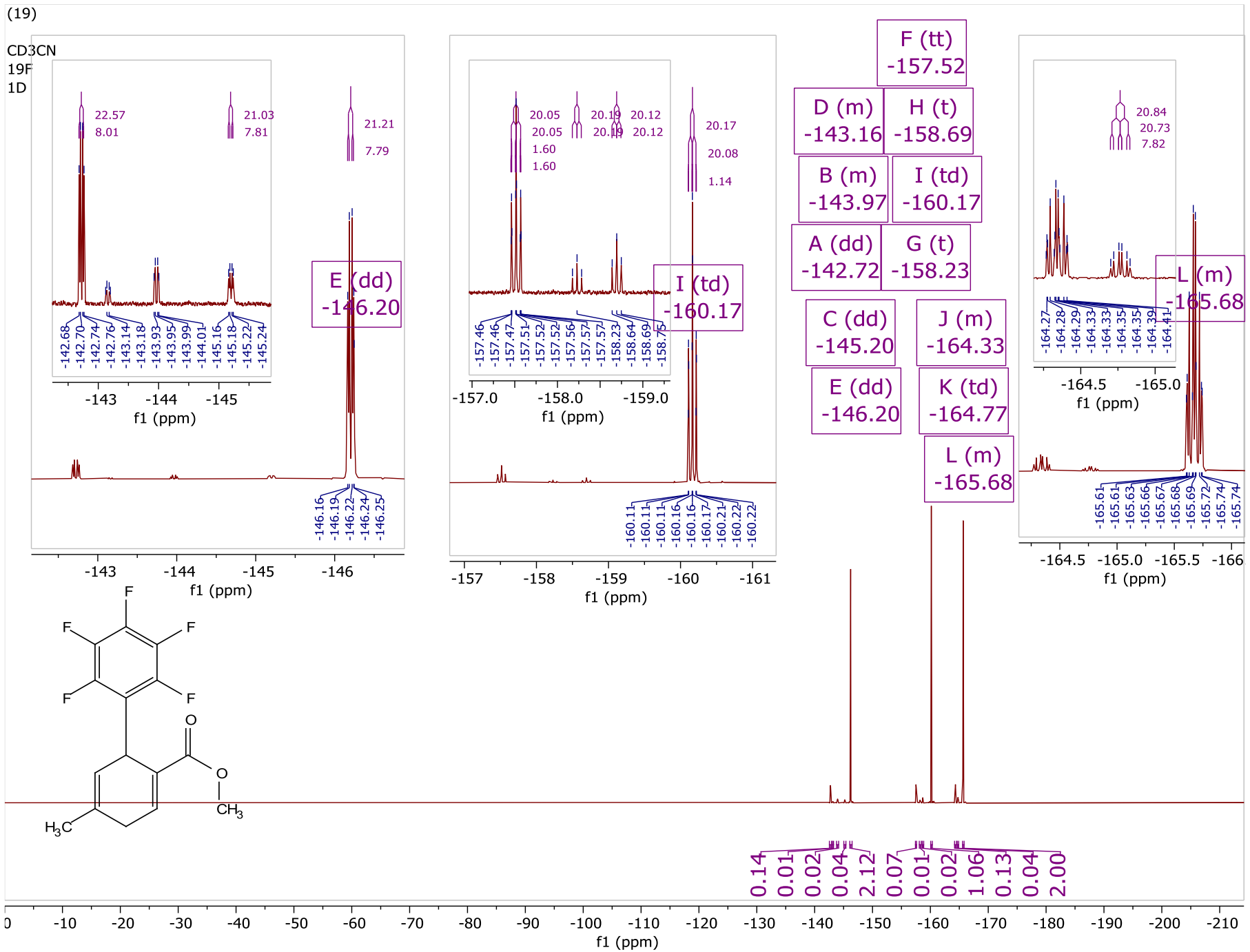


${ }^{1} \mathbf{H}$ NMR $\left(\mathrm{CD}_{3} \mathrm{CN}, 400 \mathrm{MHz}\right)$

(19)

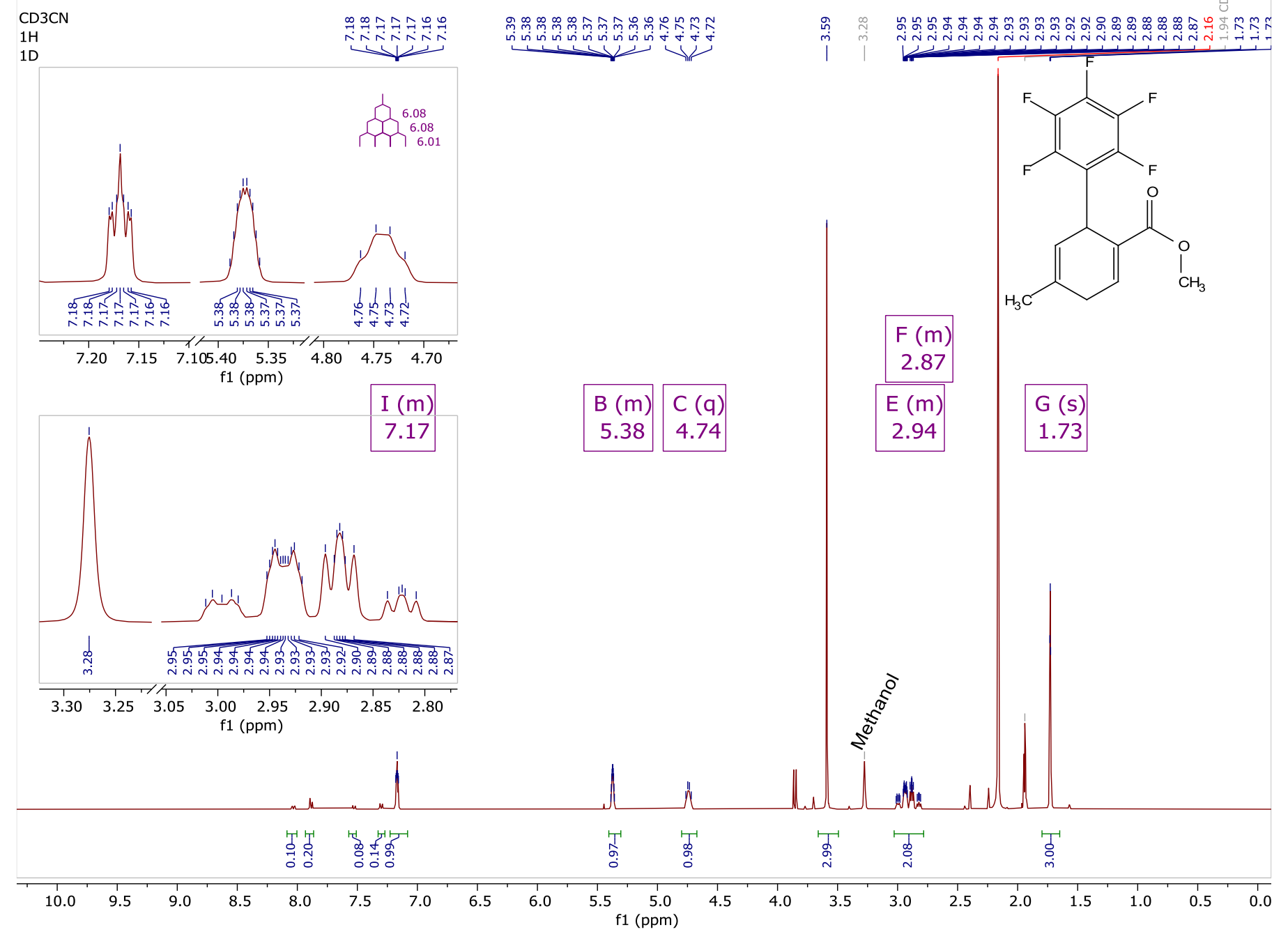




\section{${ }^{13} \mathrm{C}\left\{{ }^{1} \mathrm{H}\right\}$ NMR $\left(\mathrm{CD}_{3} \mathrm{CN}, 151 \mathrm{MHz}\right)$}

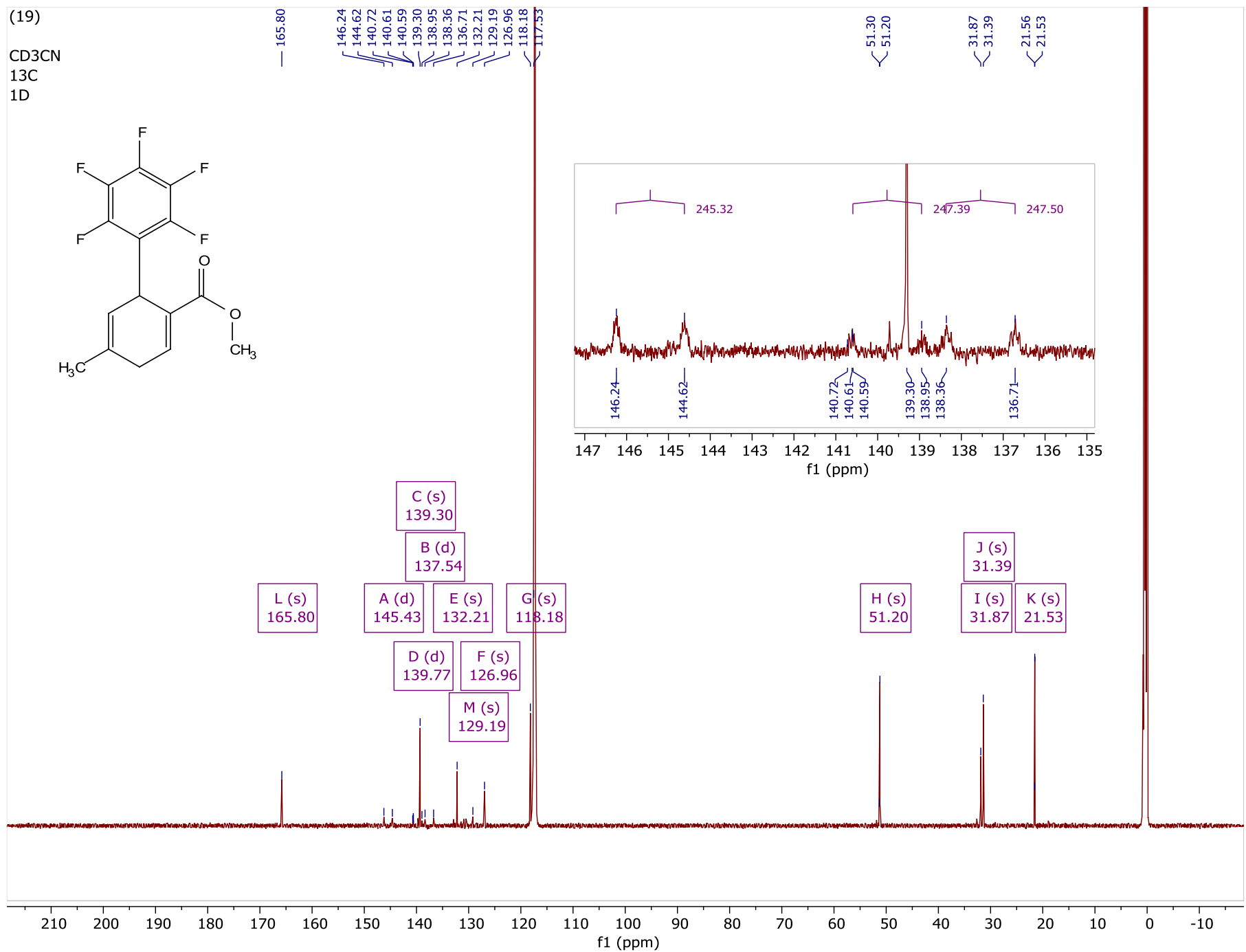


(20) methyl 4-methyl-6-(perfluoropyridin-3-yl)cyclohexa-1,4-diene-1-carboxylate

${ }^{19} \mathrm{~F}$ NMR $\left(\mathrm{CD}_{3} \mathrm{CN}, 376 \mathrm{MHz}\right)$

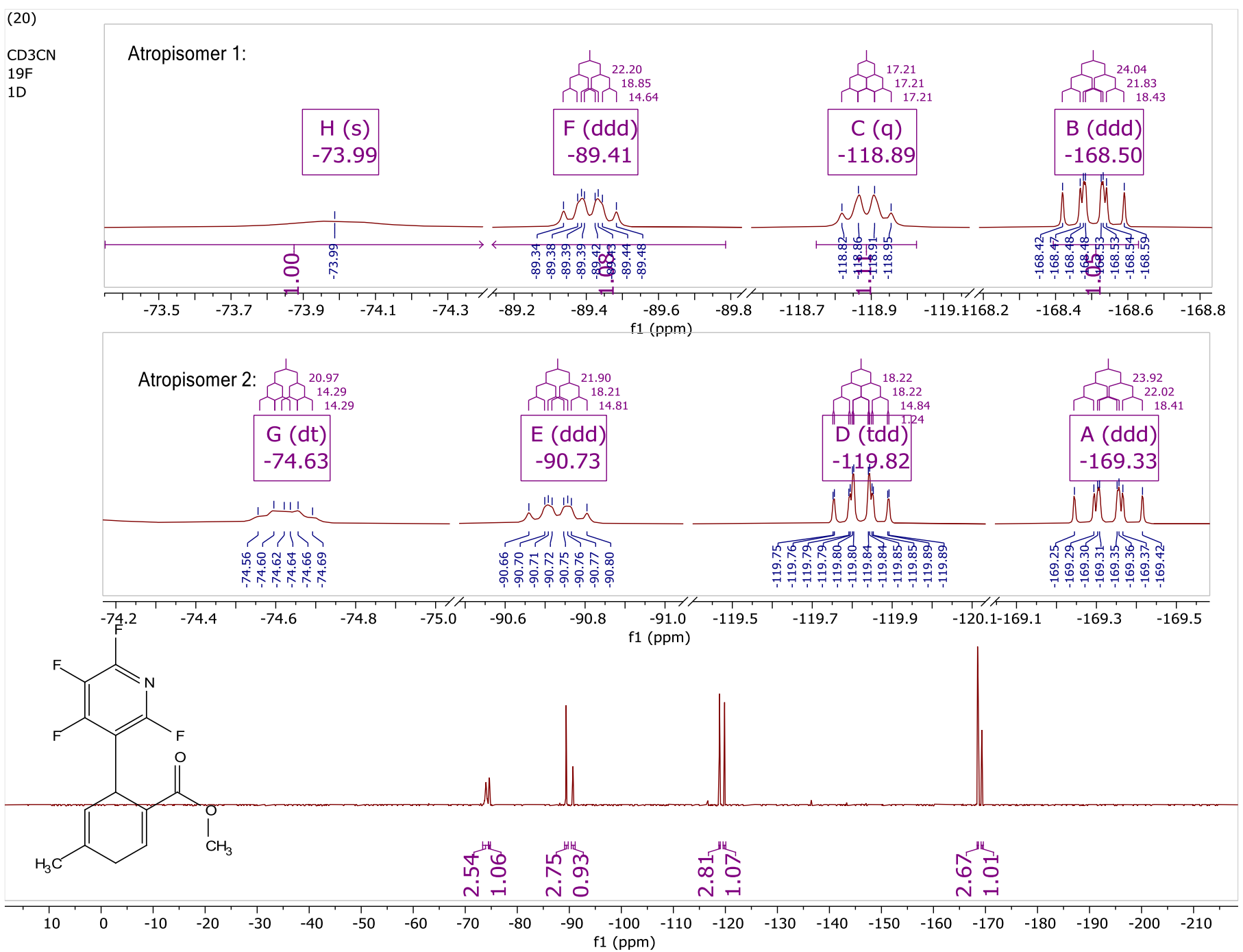


${ }^{1} \mathrm{H}$ NMR $\left(\mathrm{CD}_{3} \mathrm{CN}, 400 \mathrm{MHz}\right)$

(20)

$\mathrm{CD} 3 \mathrm{CN}$

$1 \mathrm{H}$

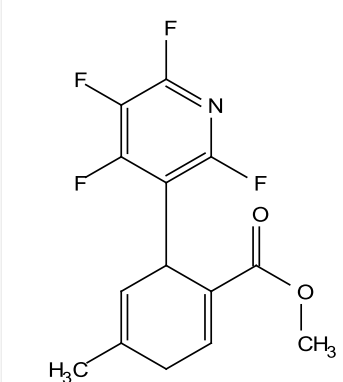

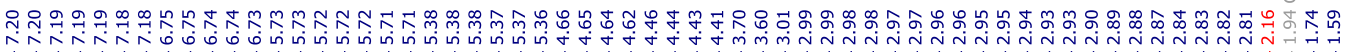
i

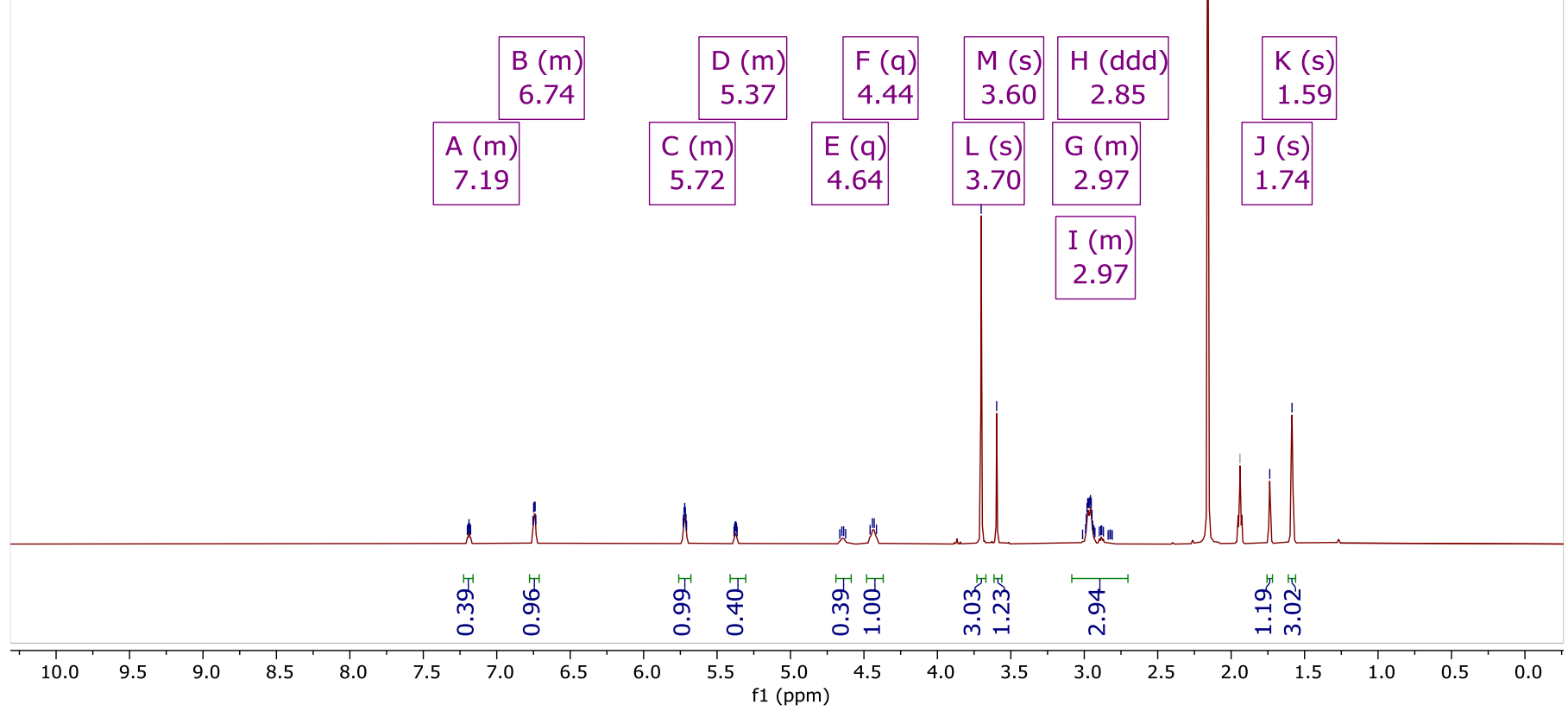


atropisomer 1

$\left\{\begin{array}{l}3.86 \\ 1.86\end{array}\right.$

M 1.86

$\widehat{\lambda} 3.33$

2.01

Im 2.01

$\overbrace{6.23}^{6.23}$

$\overbrace{}^{6.23}$

1.64

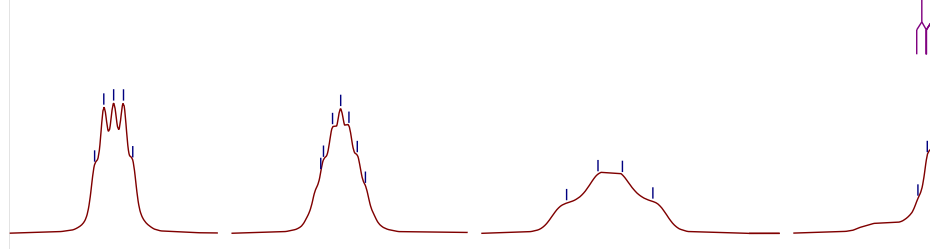

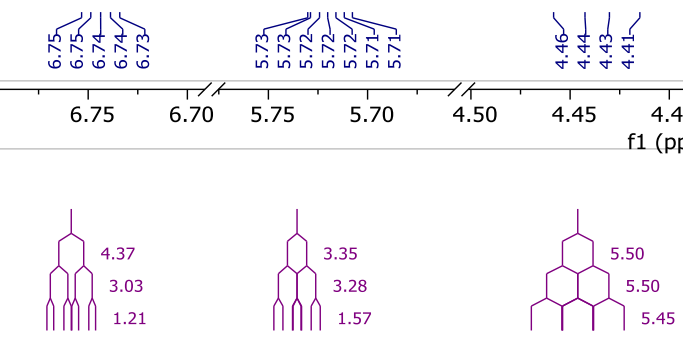

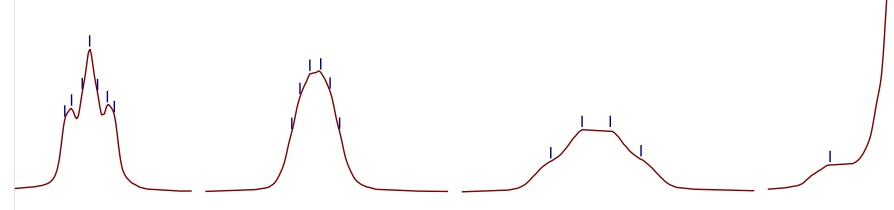

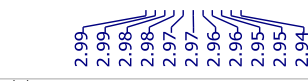

$\begin{array}{llllll}3.05 & 3.00 & 2.95 & 2.90 & 2.85 & 2.80\end{array}$

$1(\mathrm{ppm})$

$\begin{array}{lllll}3.00 & 2.95 & 2.90 & 2.85 & 2.80\end{array}$

3.723 .703 .68 f1 (ppm)

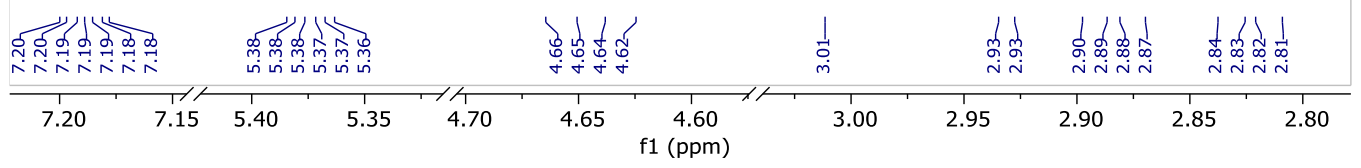
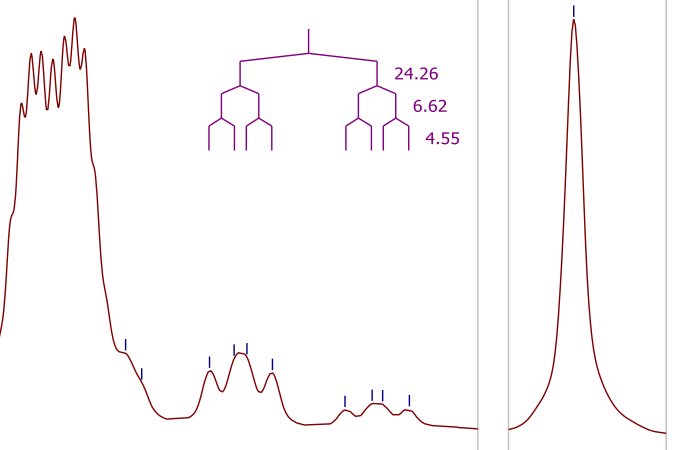

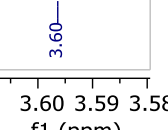
f1 (ppm)
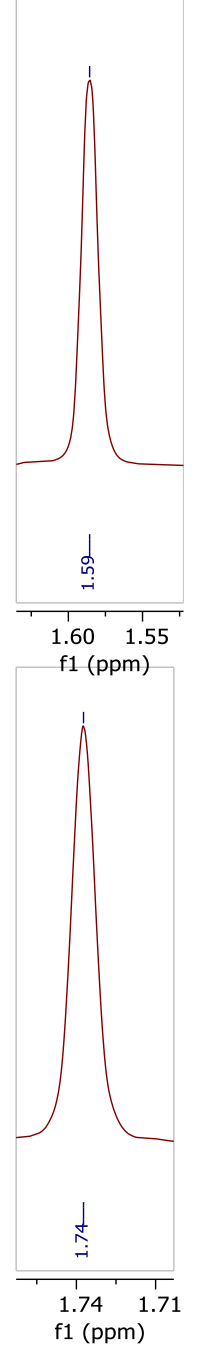
(20)

CD3CN

$1 \mathrm{H}$

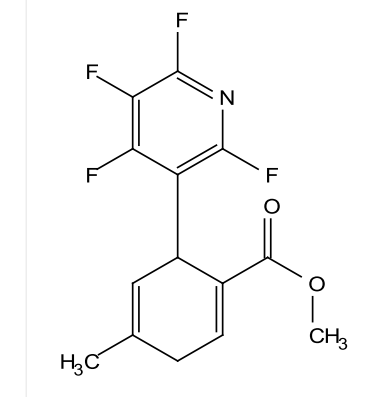

Major

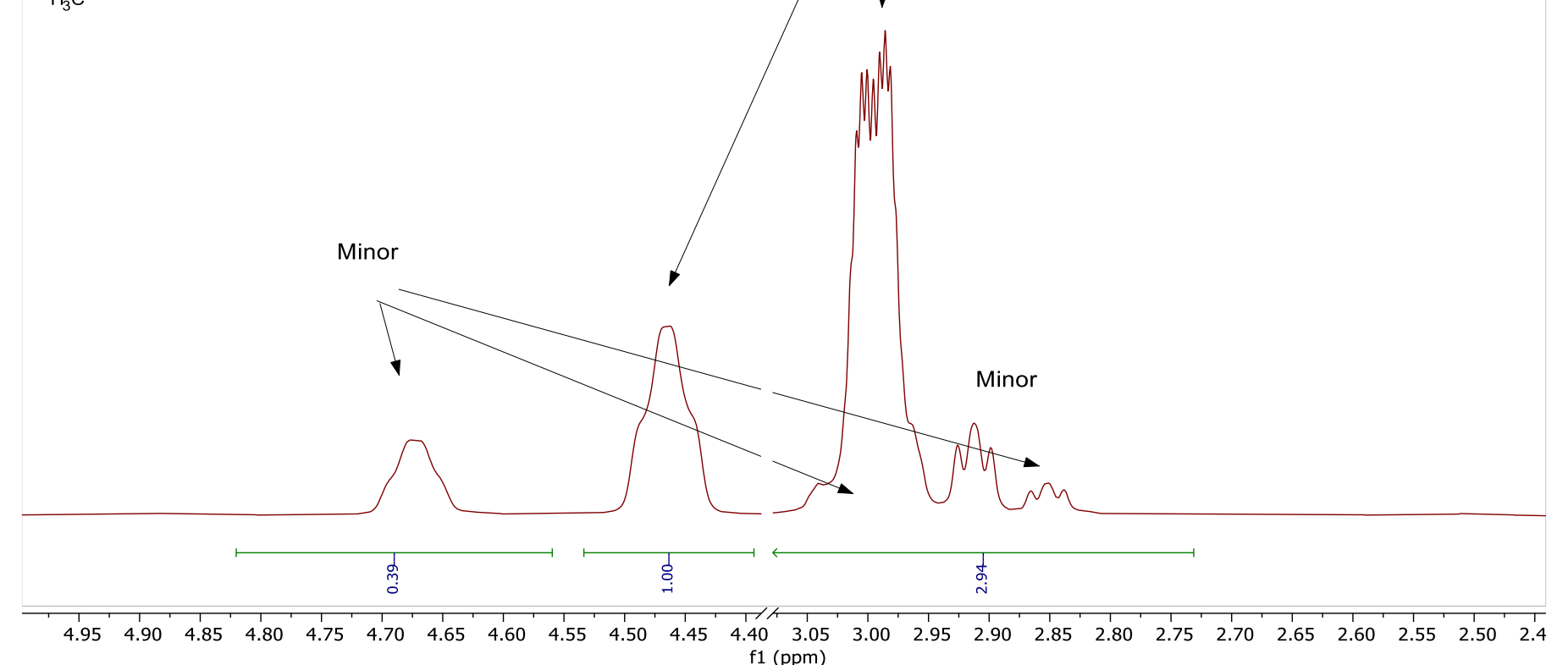


${ }^{1} \mathrm{H}-{ }^{1} \mathrm{H} \cos Y$

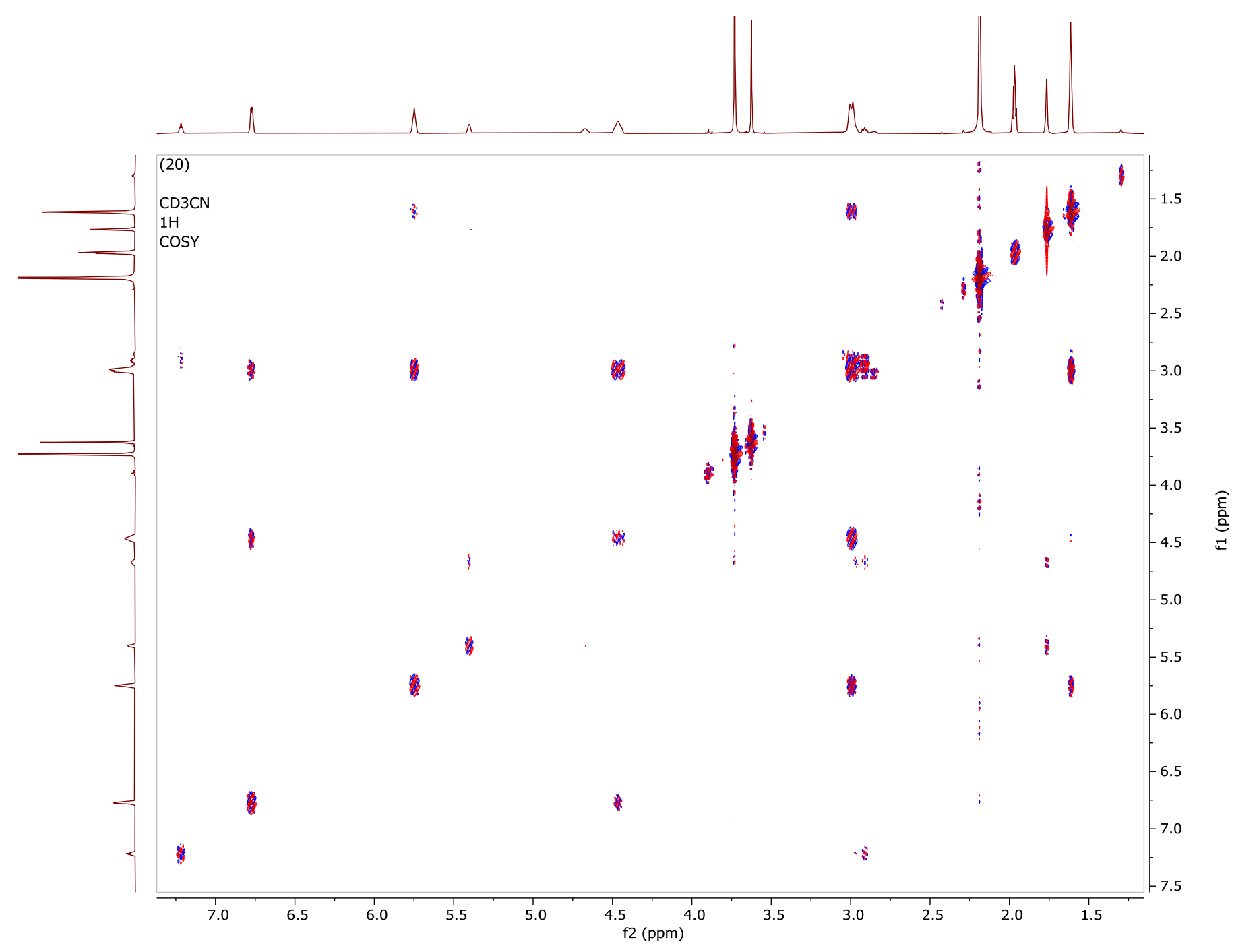


${ }^{1} \mathrm{H}-{ }^{1} \mathrm{H}$ NOESY

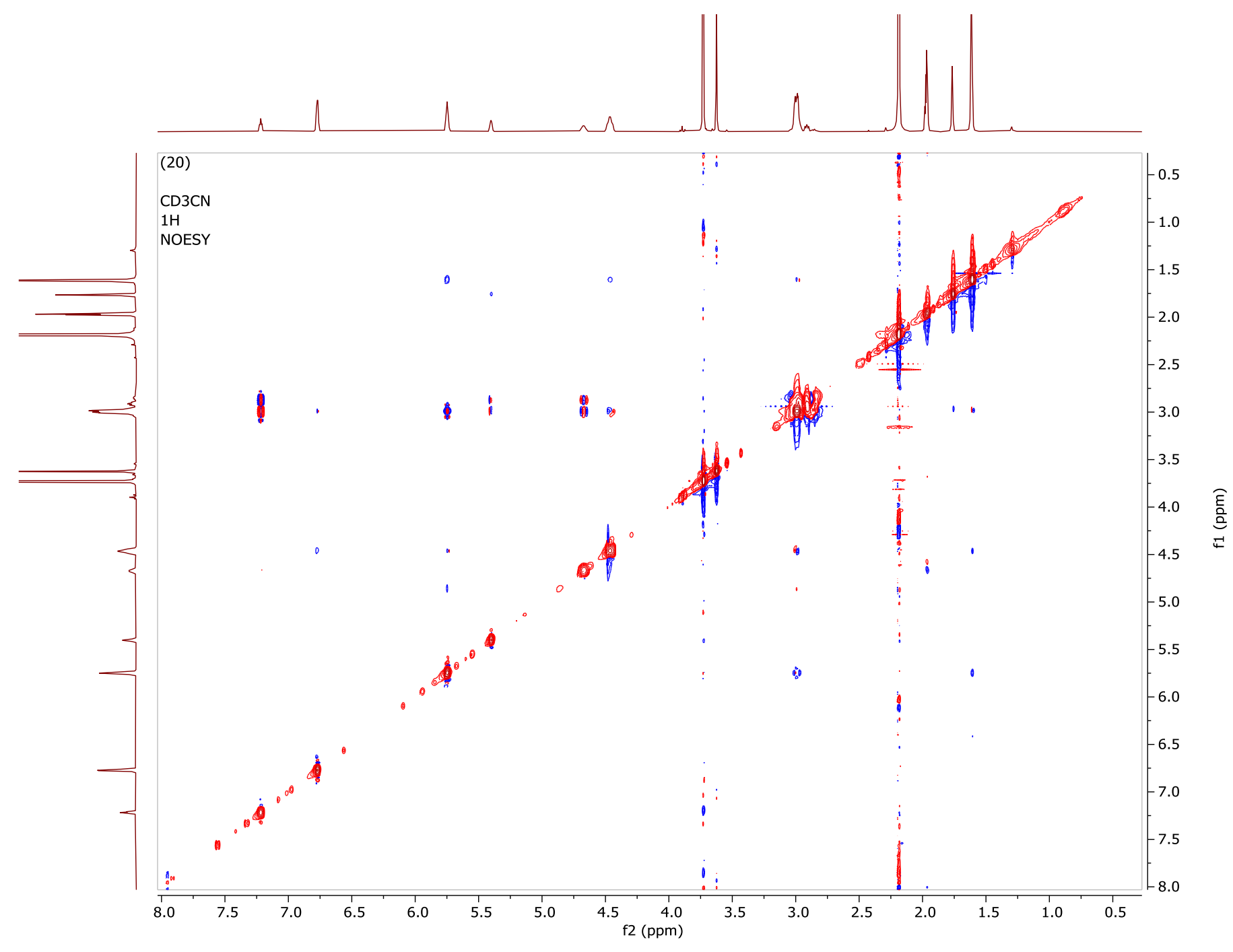


${ }^{19} \mathbf{F}$ NMR $\left(\mathrm{CDCl}_{3}, 376 \mathrm{MHz}\right)$
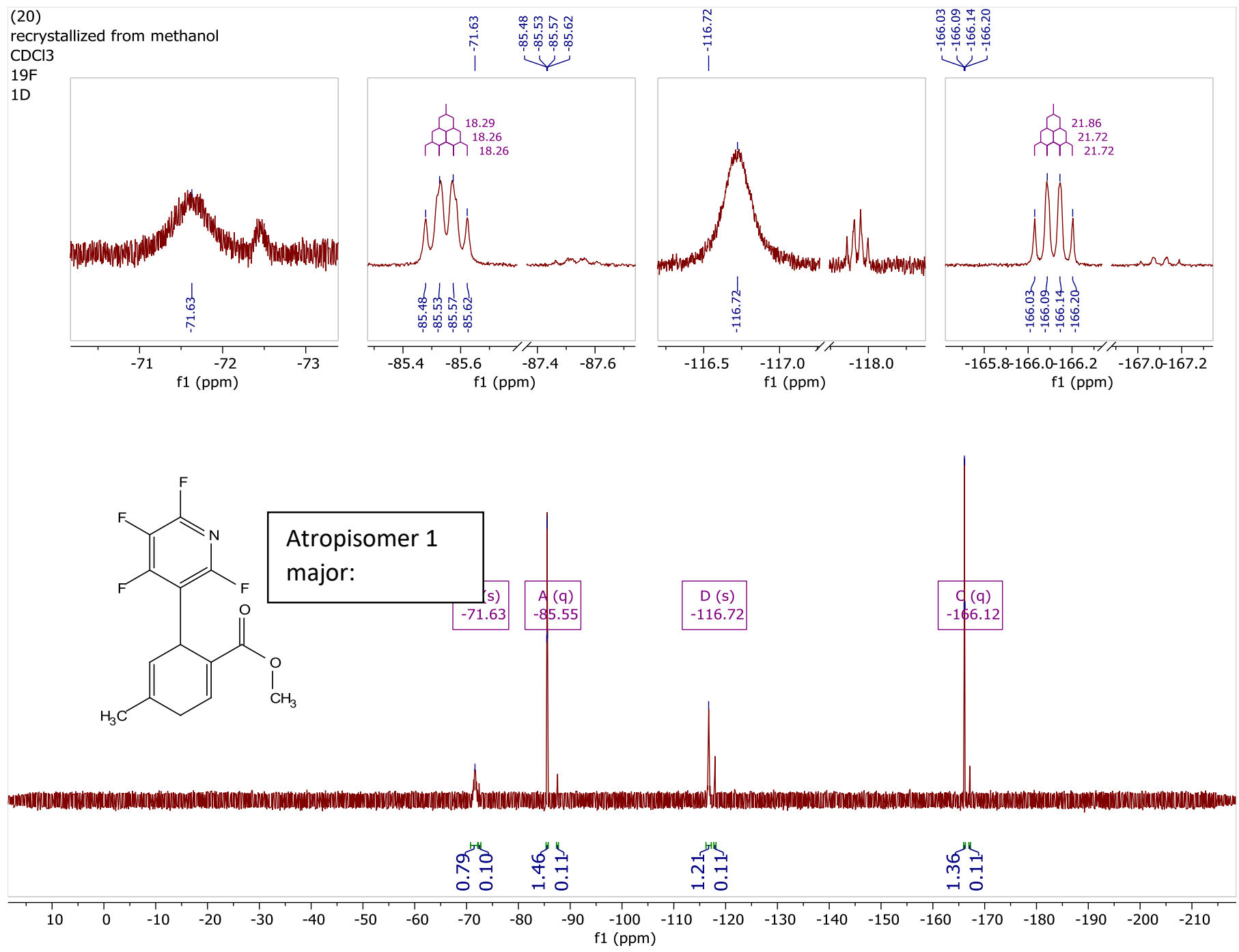
${ }^{19}$ F NMR $\left(\mathrm{CDCl}_{3}, 376 \mathrm{MHz}\right)$

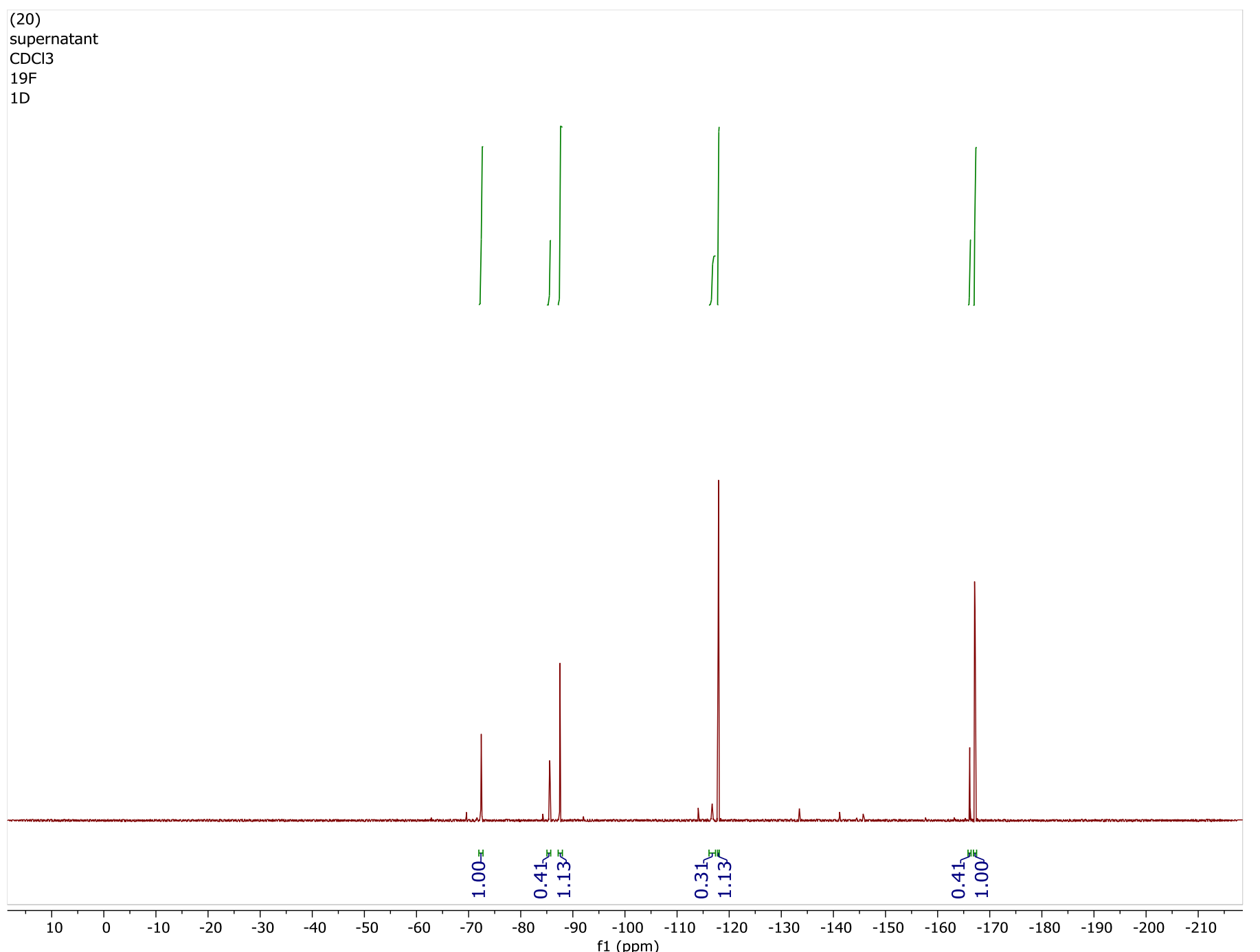


${ }^{1} \mathbf{H}$ NMR $\left(\mathrm{CDCl}_{3}, 400 \mathrm{MHz}\right)$

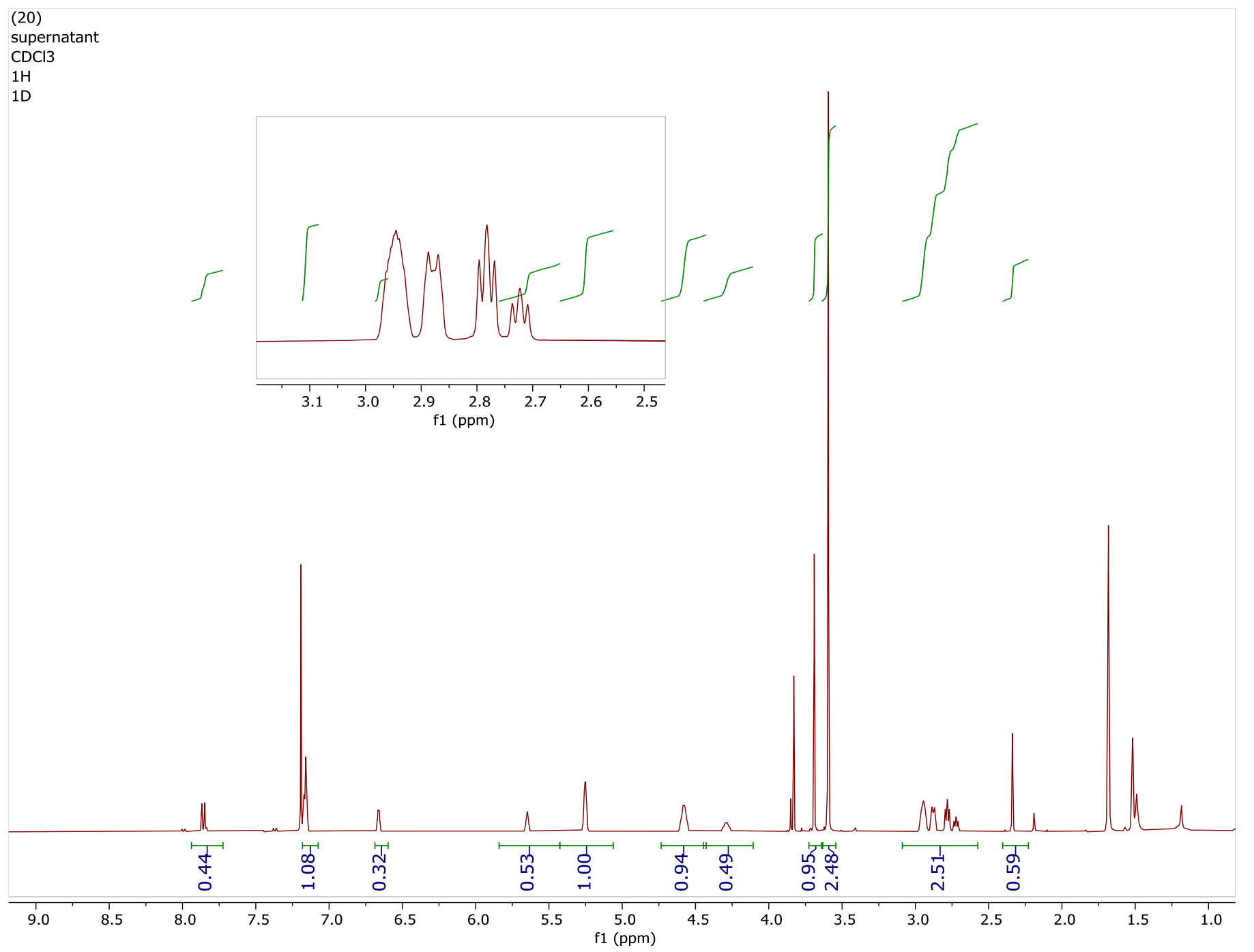


${ }^{\mathbf{1}} \mathbf{H} \mathbf{N M R}\left(\mathrm{CDCl}_{3}, 400 \mathrm{MHz}\right)$

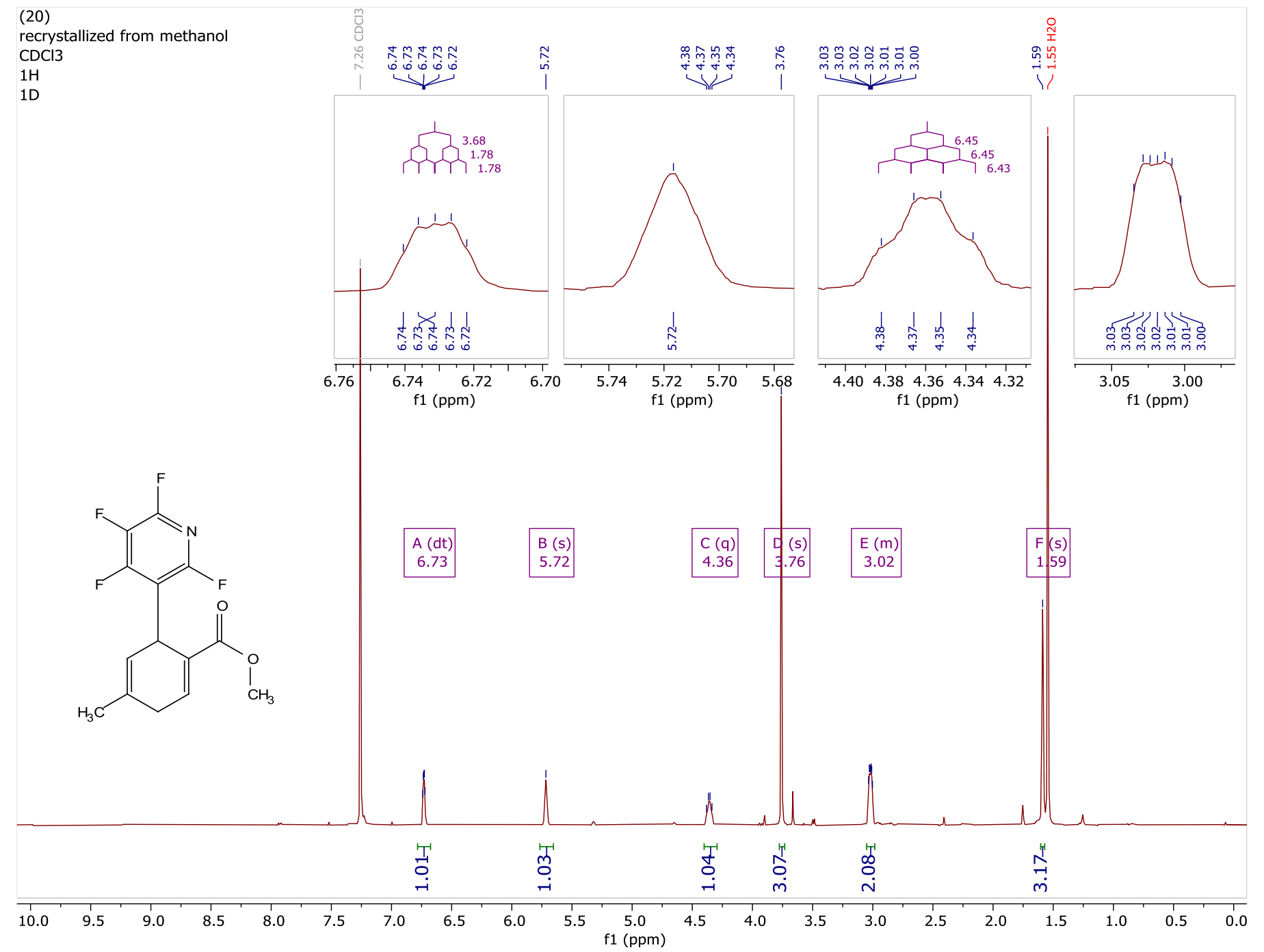


${ }^{13} \mathrm{C}\left\{{ }^{1} \mathrm{H}\right\}$ NMR $\left(\mathrm{CD}_{3} \mathrm{CN}, 151 \mathrm{MHz}\right)$

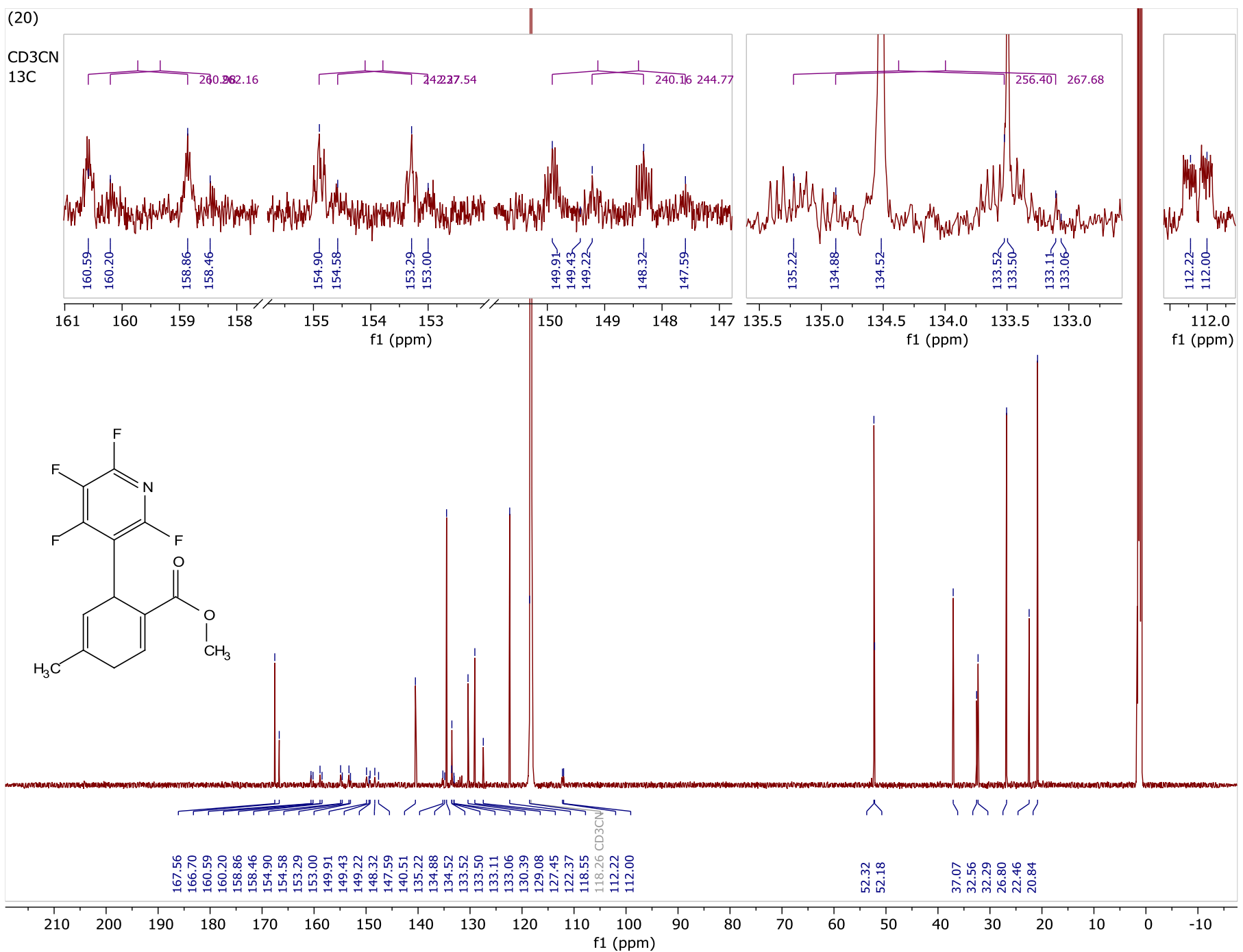


${ }^{1} \mathrm{H}-{ }^{1} \mathrm{H}$ HMBC

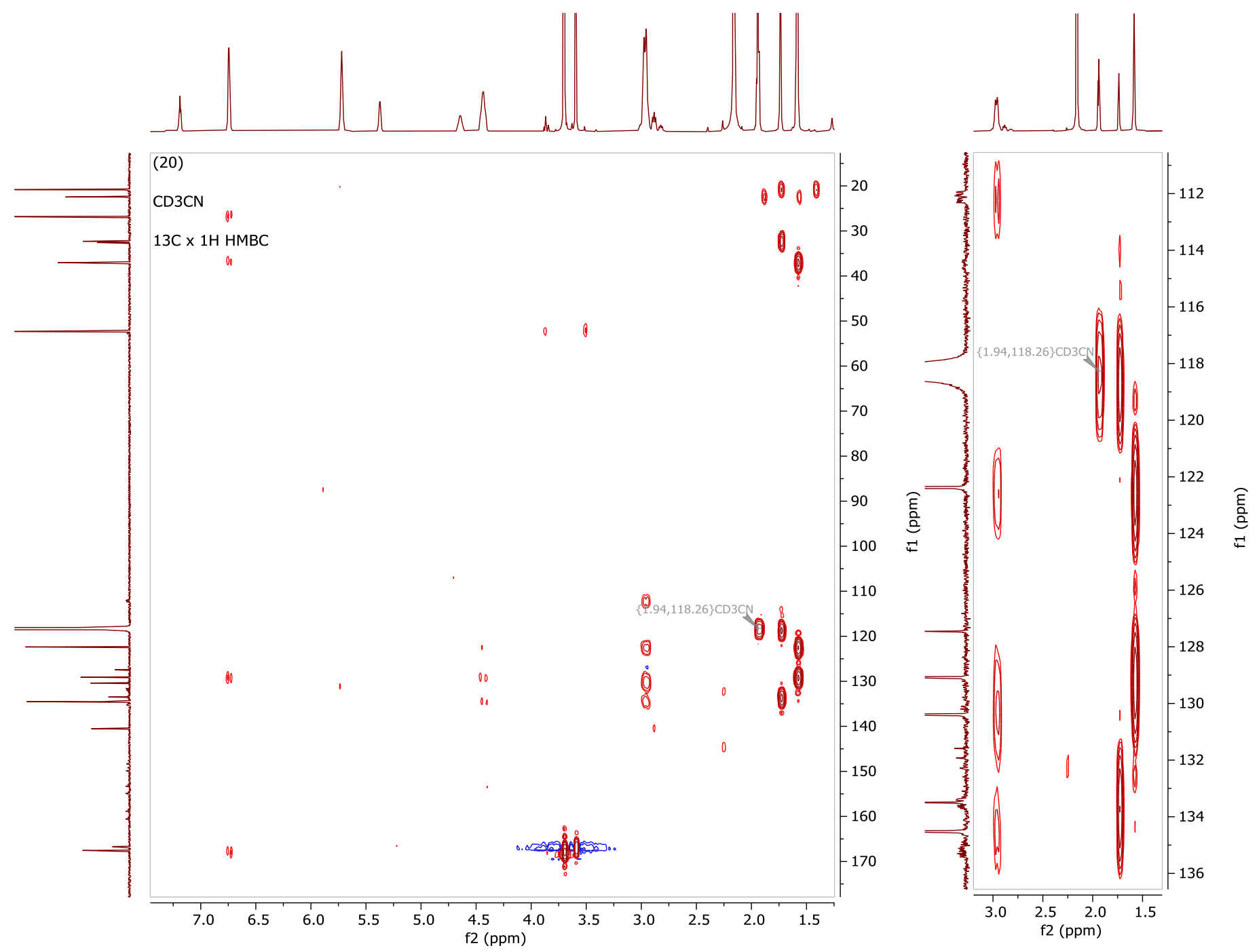


(21) Methyl 4' -cyano-2',3',5',6'-tetrafluoro-5-oxo-1,4,5,6-tetrahydro-[1,1'-biphenyl]-2-carboxylate

${ }^{19} \mathbf{F}$ NMR $\left(\mathrm{CDCl}_{3}, 376 \mathrm{MHz}\right)$

(21)

$\mathrm{CDCl} 3$

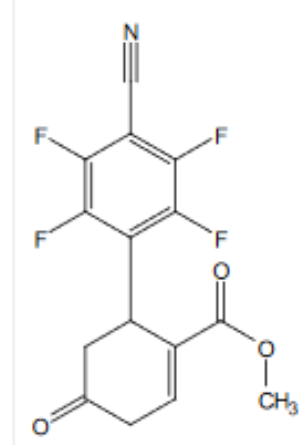

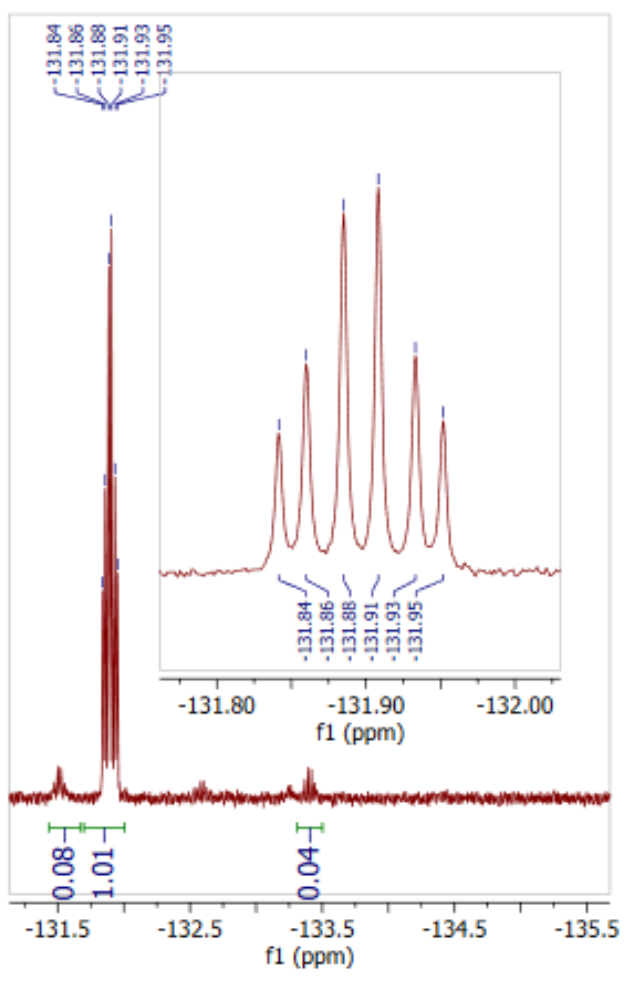

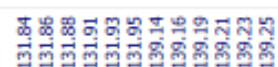

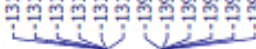

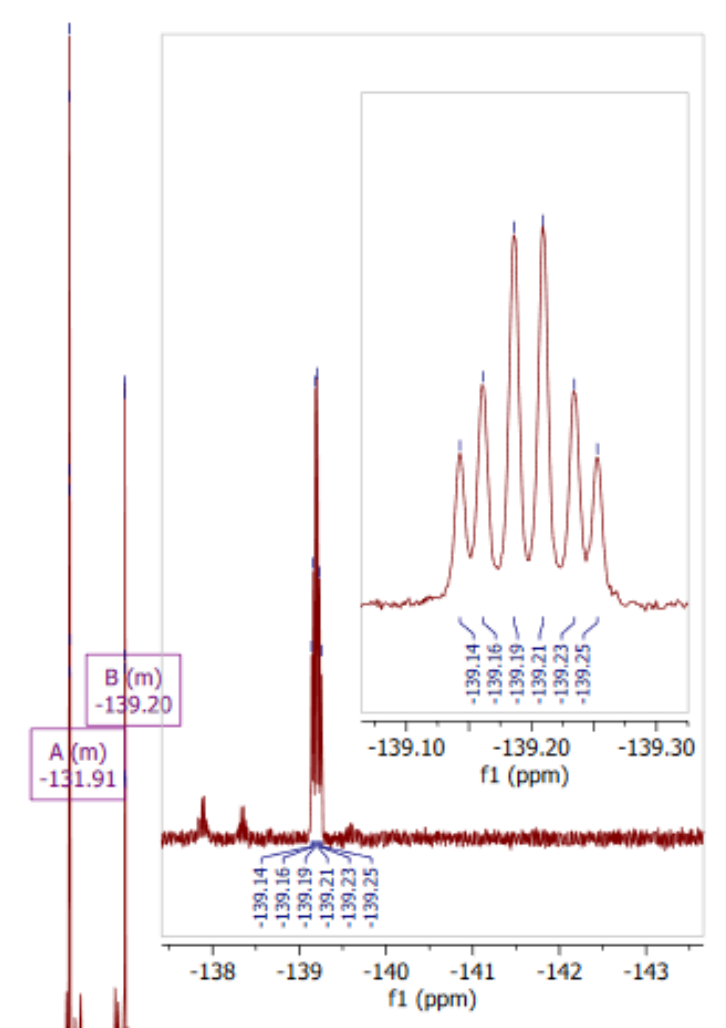

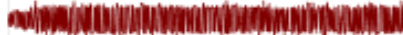

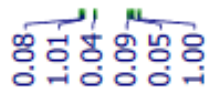

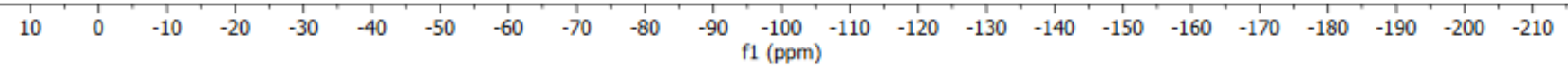


${ }^{\mathbf{1}} \mathbf{H} \mathbf{N M R}\left(\mathrm{CDCl}_{3}, 400 \mathrm{MHz}\right)$

(22)

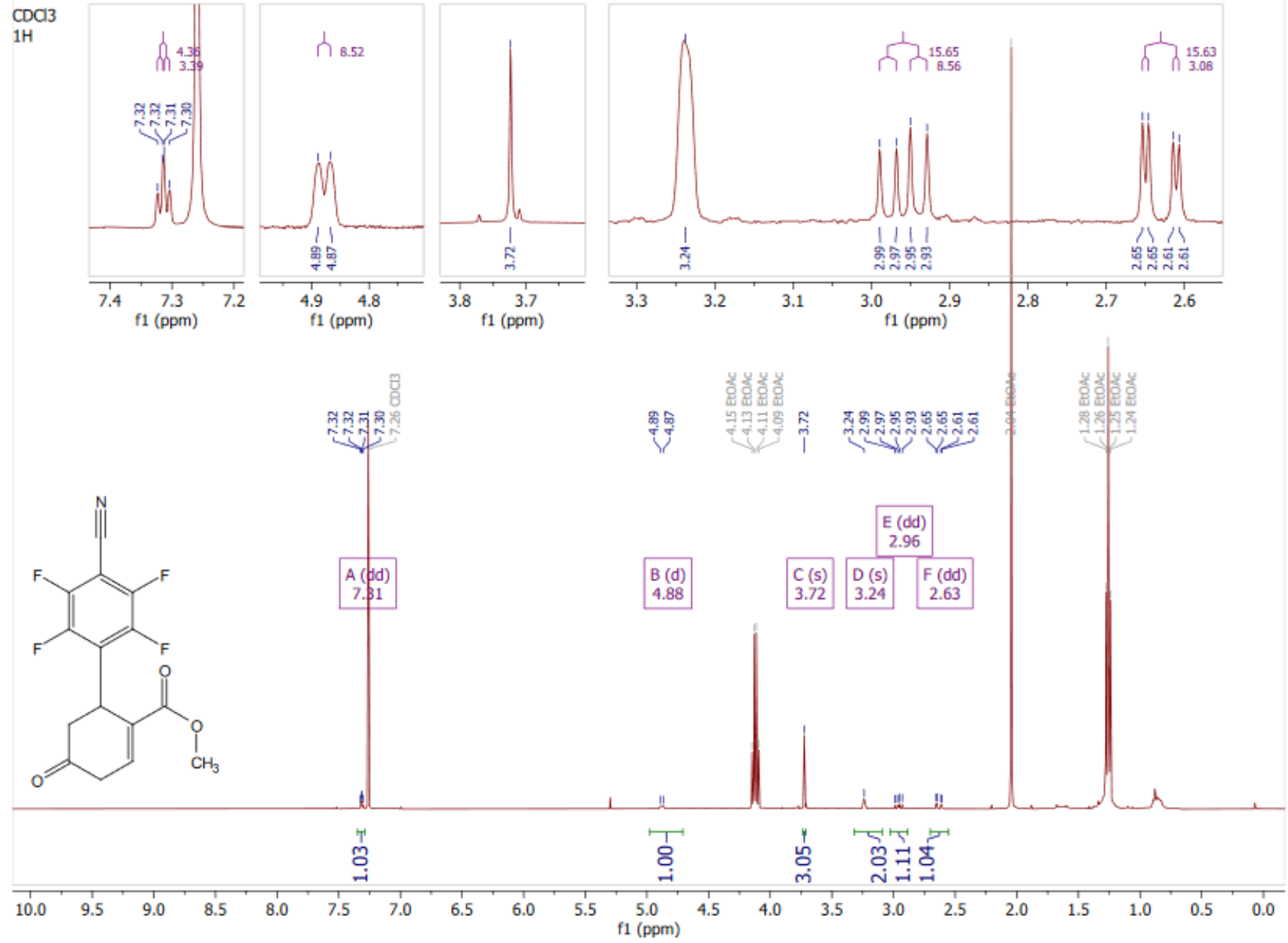


${ }^{1} \mathbf{H}$ NMR $\left(\mathrm{CD}_{3} \mathrm{CN}, 400 \mathrm{MHz}\right)$

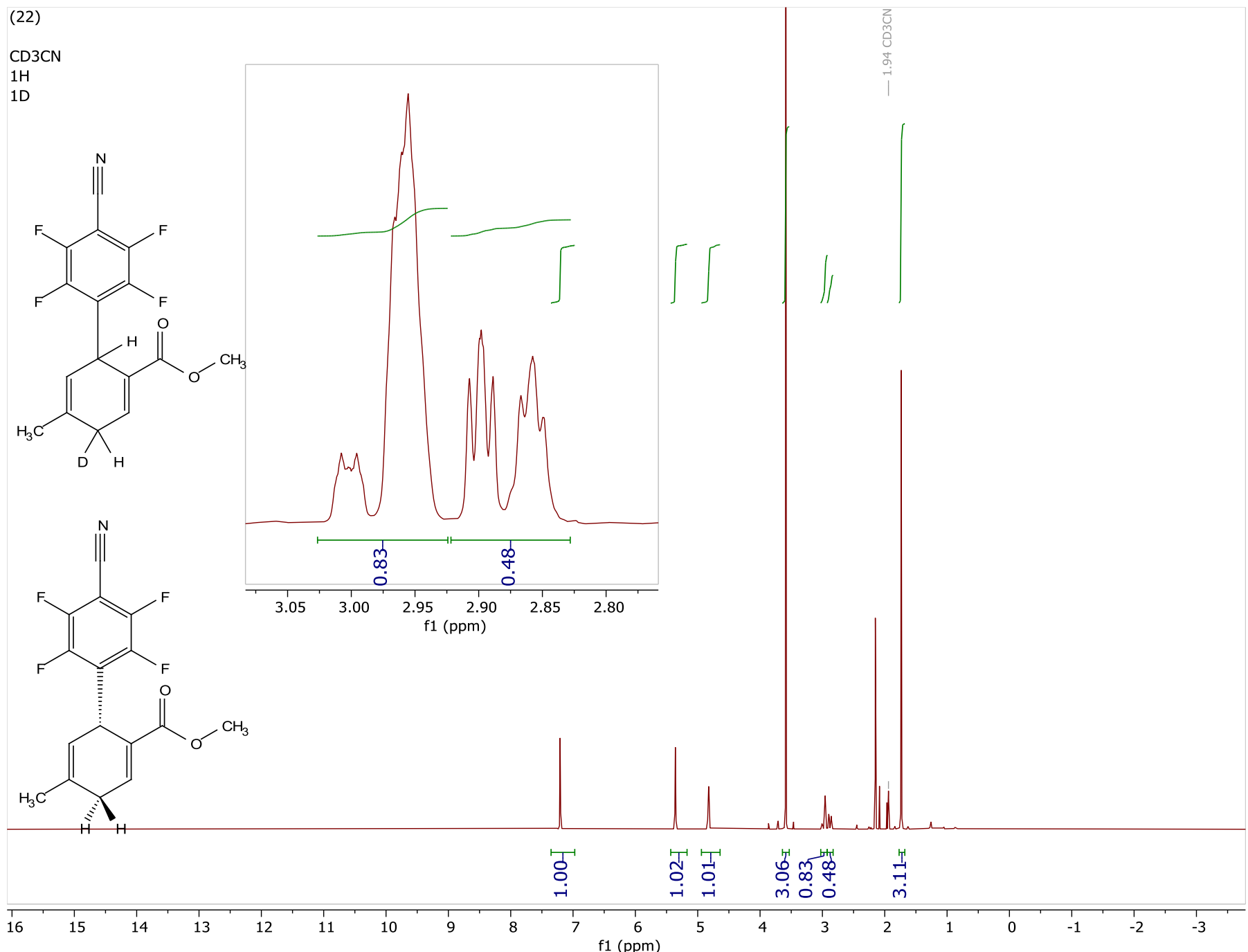


${ }^{1} \mathbf{H}$ NMR $\left(\mathrm{CD}_{3} \mathrm{CN}, 400 \mathrm{MHz}\right)$

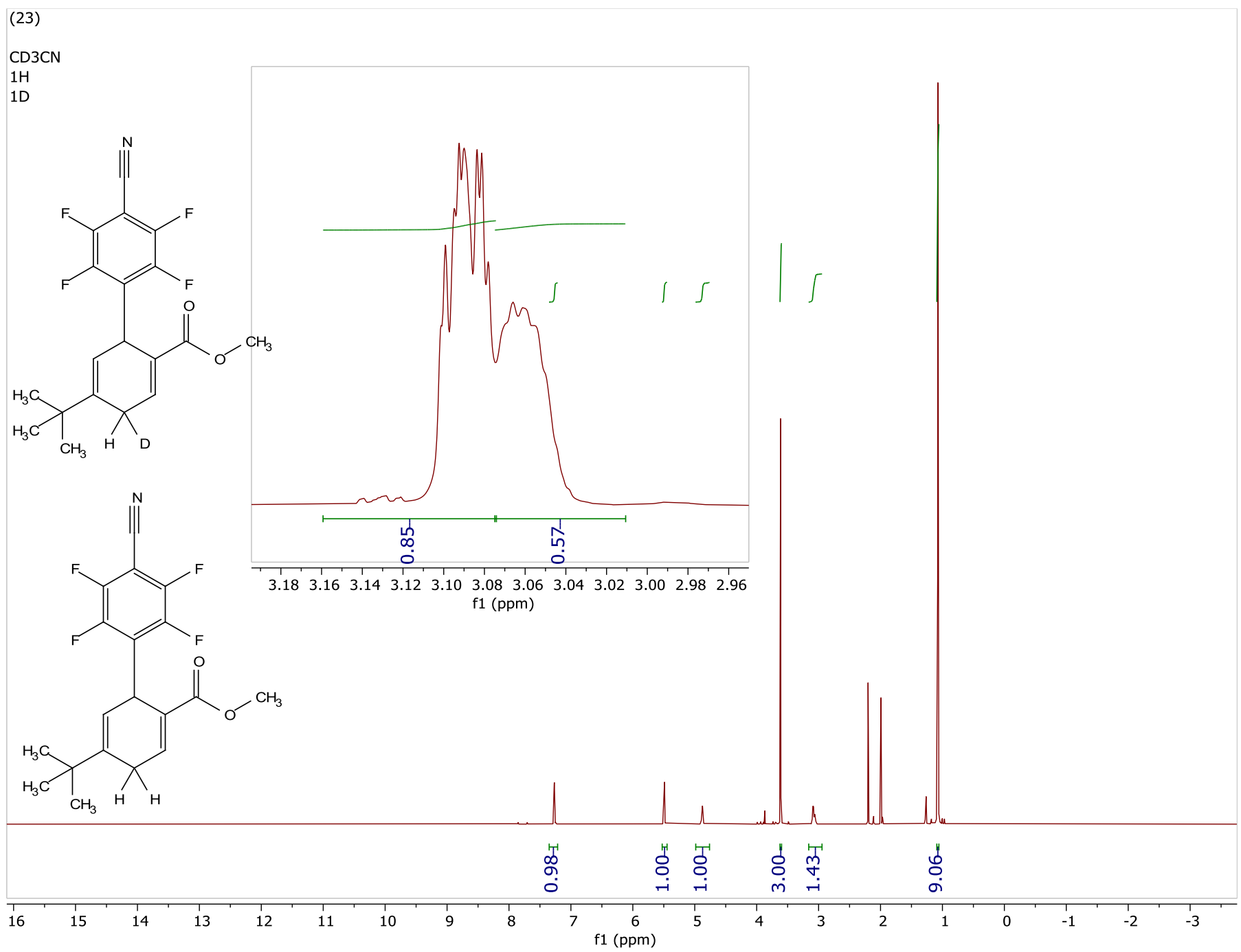


(24) Methyl 2',3',5',6' -tetrafluoro-4' -(1-hydroxypentyl)-5-methyl-1,4-dihydro-[1,1' - biphenyl]-2-carboxylate

${ }^{19}$ F NMR $\left(\mathrm{CDCl}_{3}, 376 \mathrm{MHz}\right)$

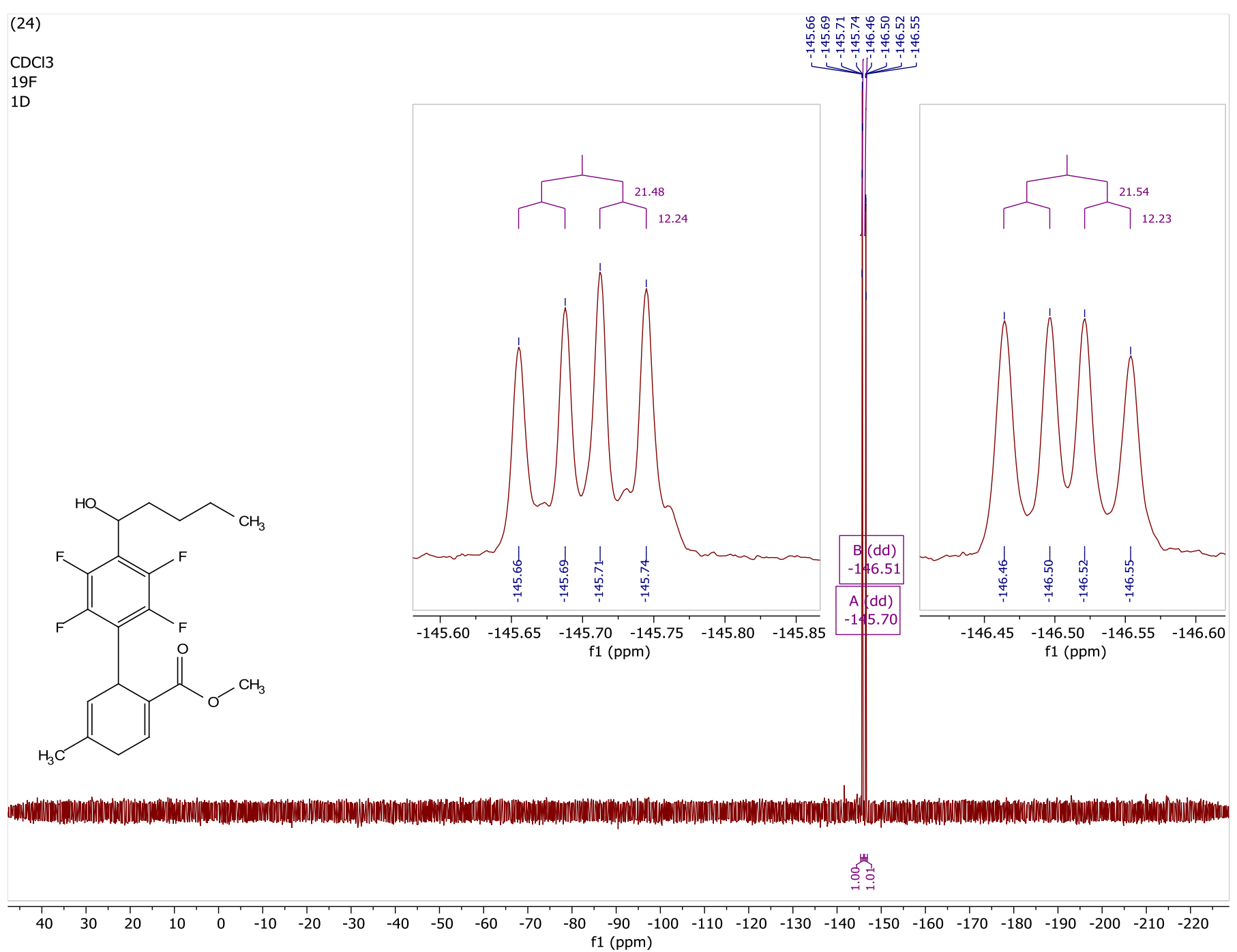


'1H NMR $\left(\mathrm{CDCl}_{3}, 400 \mathrm{MHz}\right)$

(24)

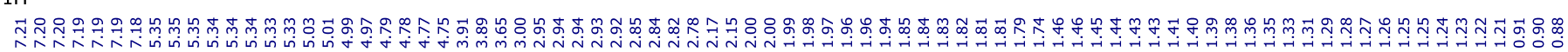
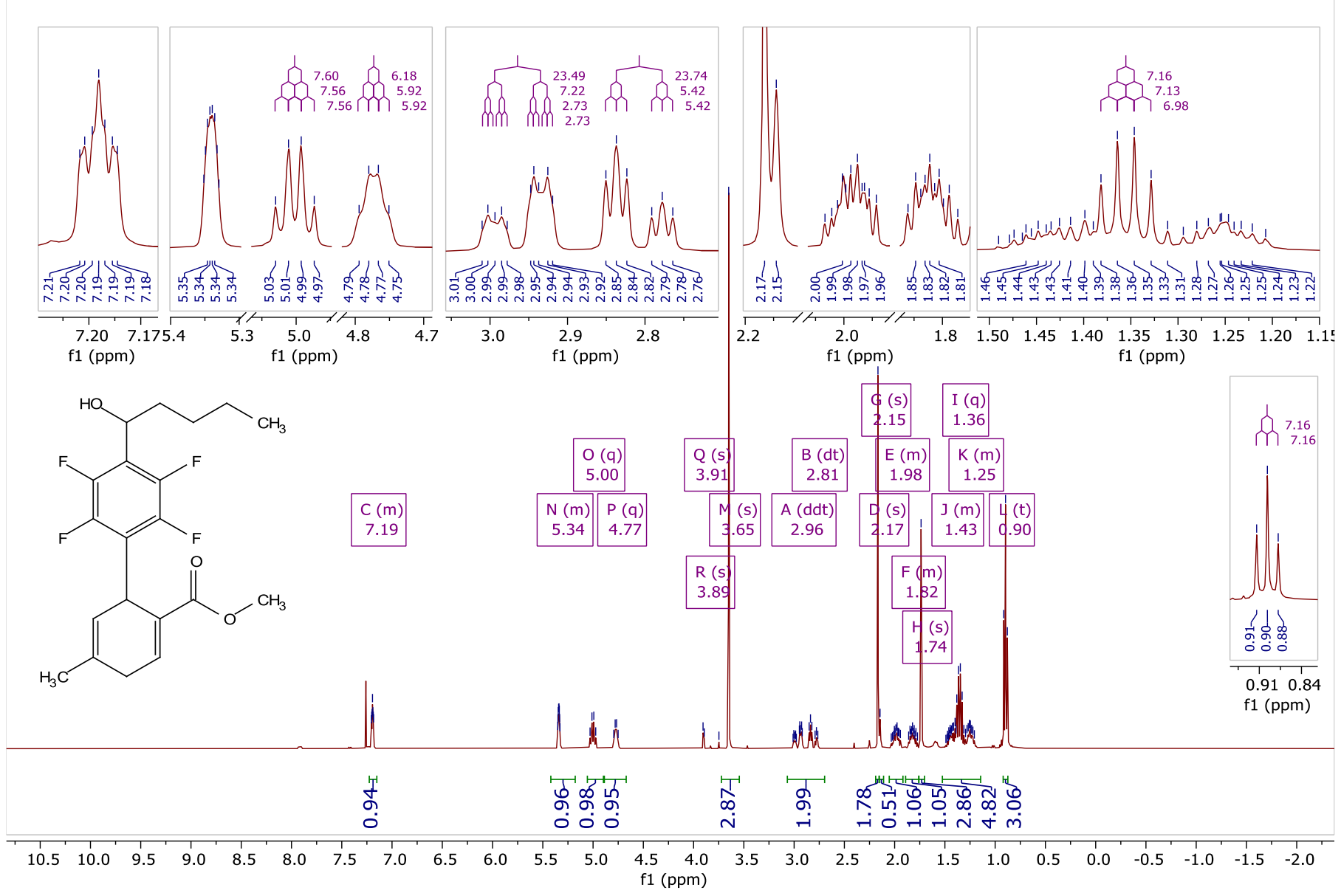


\section{${ }^{13} \mathrm{C}\left\{{ }^{1} \mathrm{H}\right\}$ NMR $\left(\mathrm{CDCl}_{3}, 101 \mathrm{MHz}\right)$}

(24)

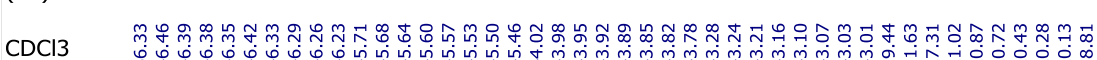

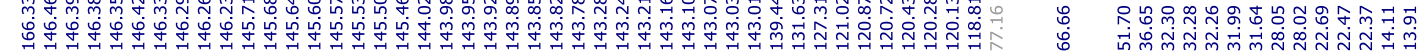

I
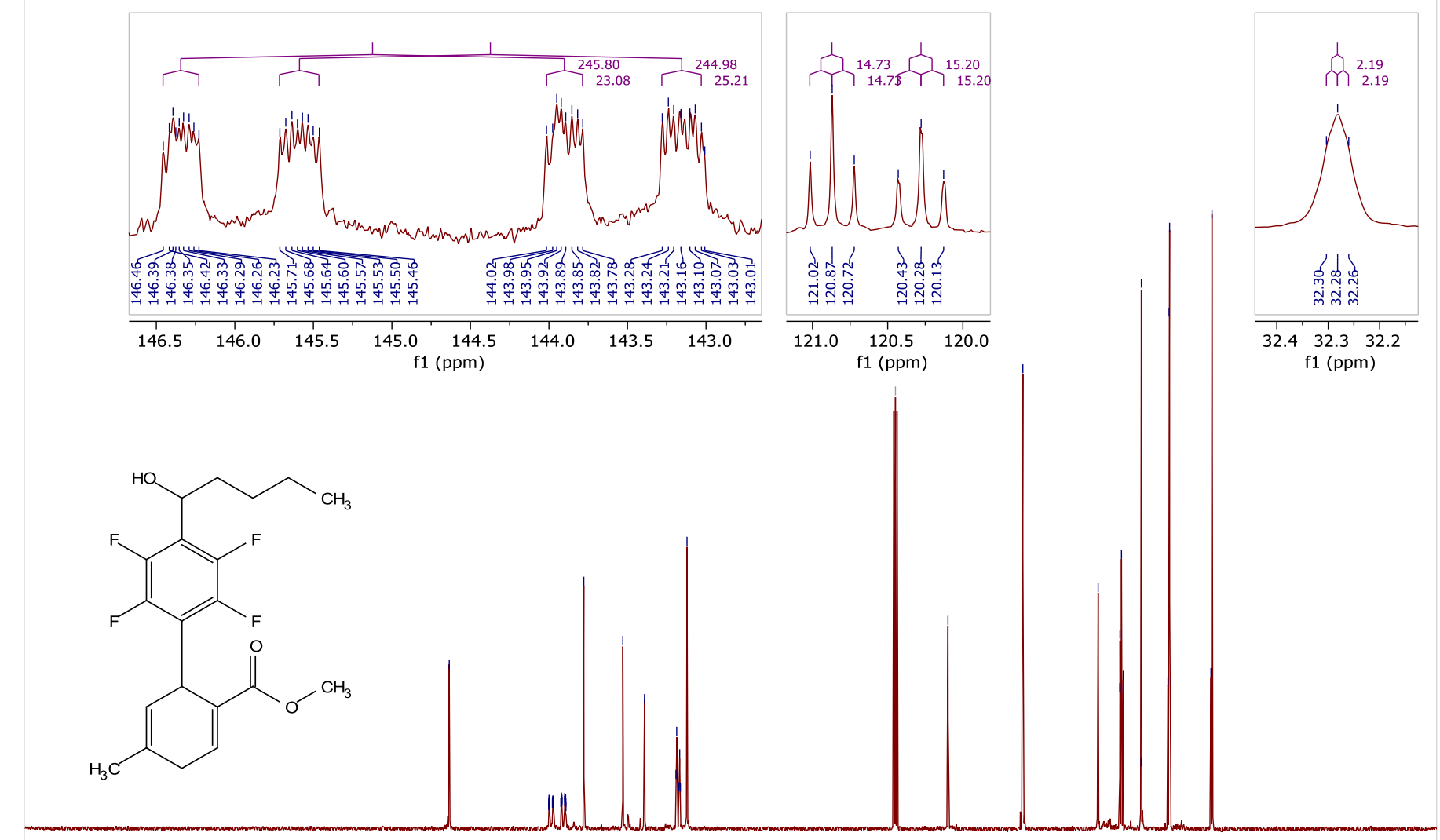

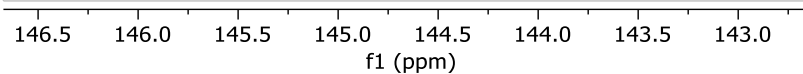

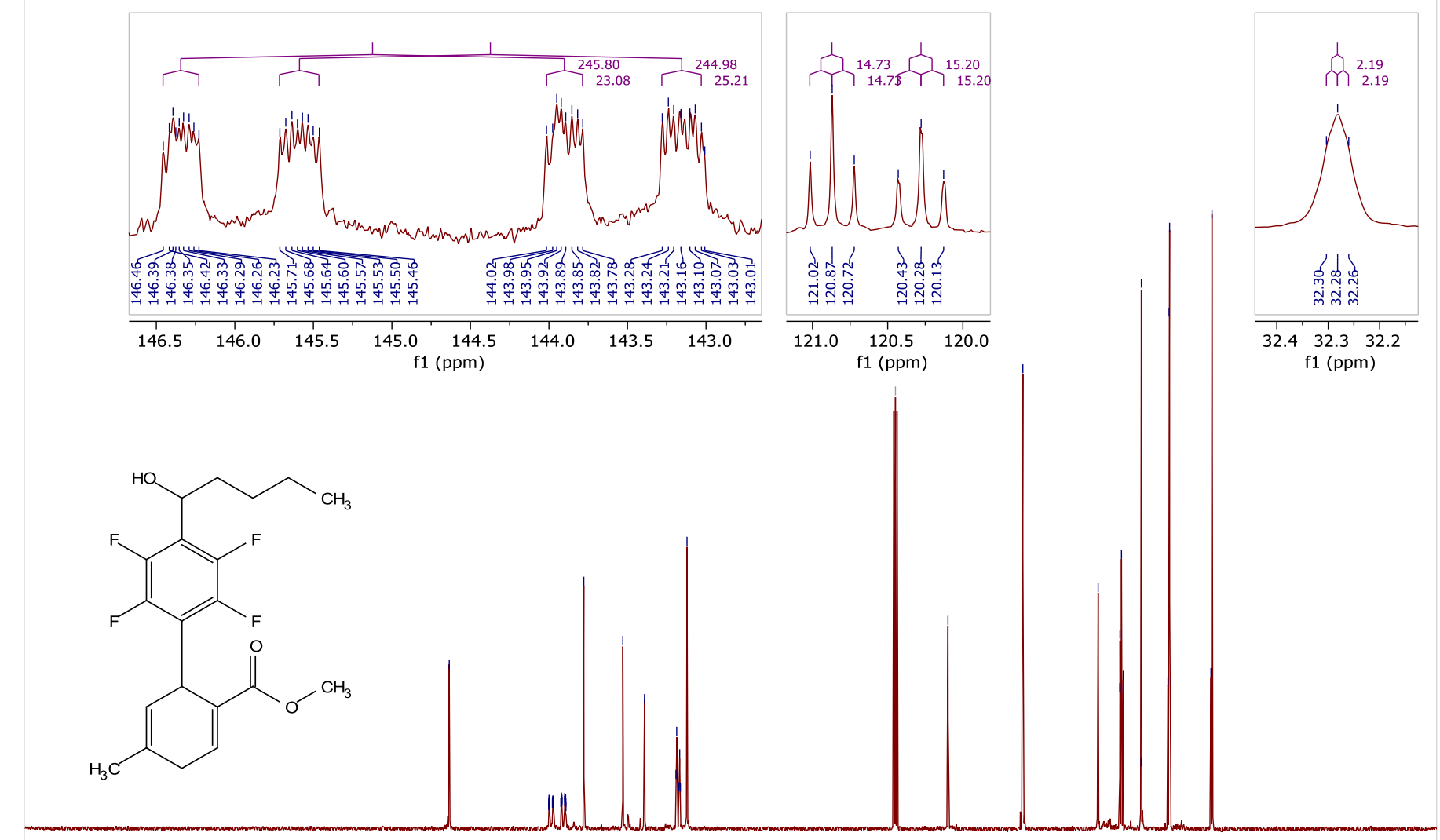
$\begin{array}{lllllllllllllllllllllllllllllllllllllllllll}5 & 50 & 240 & 230 & 220 & 210 & 200 & 190 & 180 & 170 & 160 & 150 & 140 & 130 & 120 & 110 & 100 & 90 & 80 & 70 & 60 & 50 & 40 & 30 & 20 & 10 & 0 & -10 & -20 & -3\end{array}$ 
(25) Methyl 4'-(1-((3,5-bis(trifluoromethyl)benzoyl)oxy)pentyl)-2',3',5',6'-tetrafluoro-5- methyl-1,4-dihydro-[1,1'-biphenyl]-2-carboxylate ${ }^{19} \mathrm{~F}$ NMR $\left(\mathrm{CDCl}_{3}, 376 \mathrm{MHz}\right)$

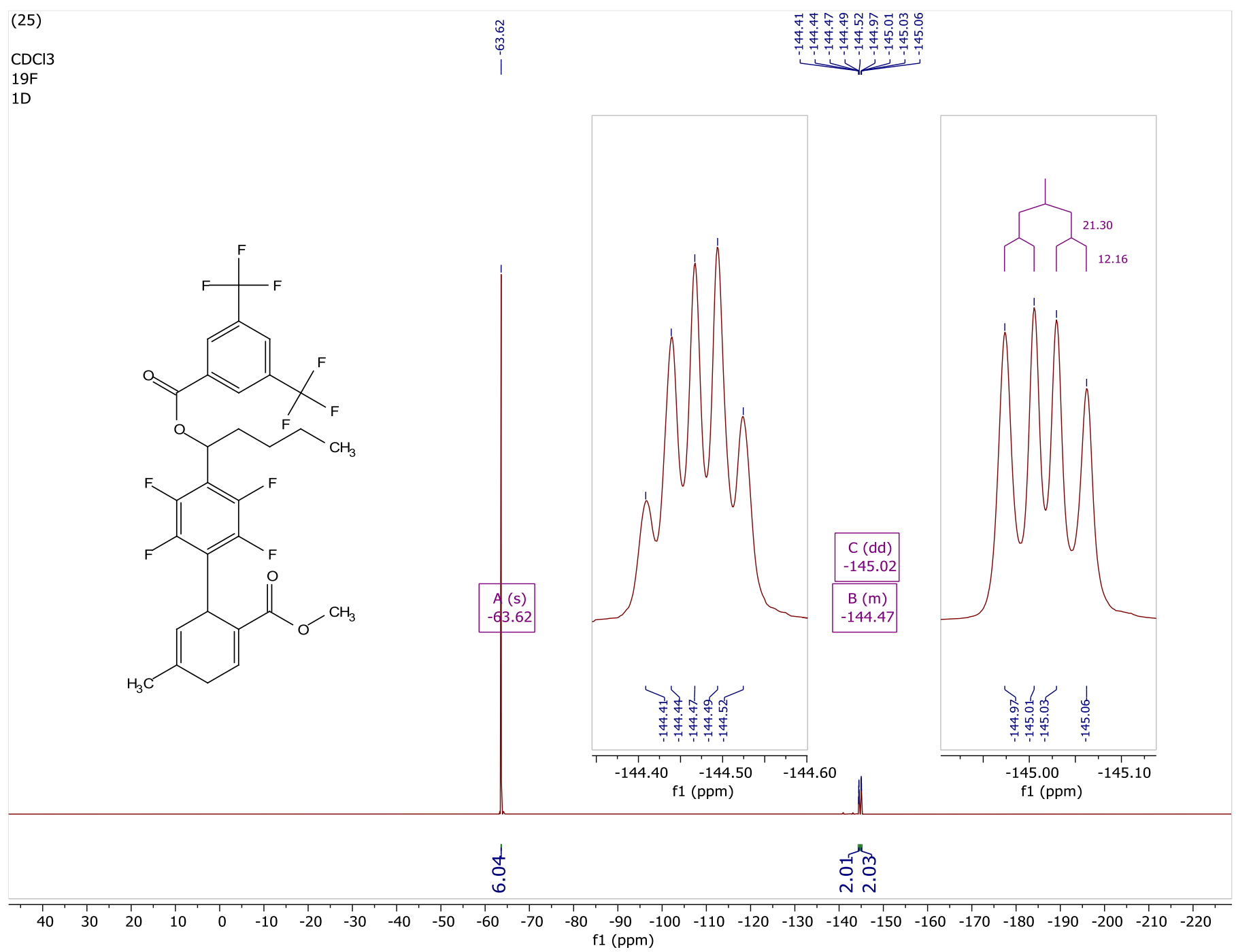


${ }^{1} \mathbf{H}$ NMR $\left(\mathrm{CDCl}_{3}, 400 \mathrm{MHz}\right)$

$(25)$
$\mathrm{CDCl} 3$

$\underbrace{1 H}$
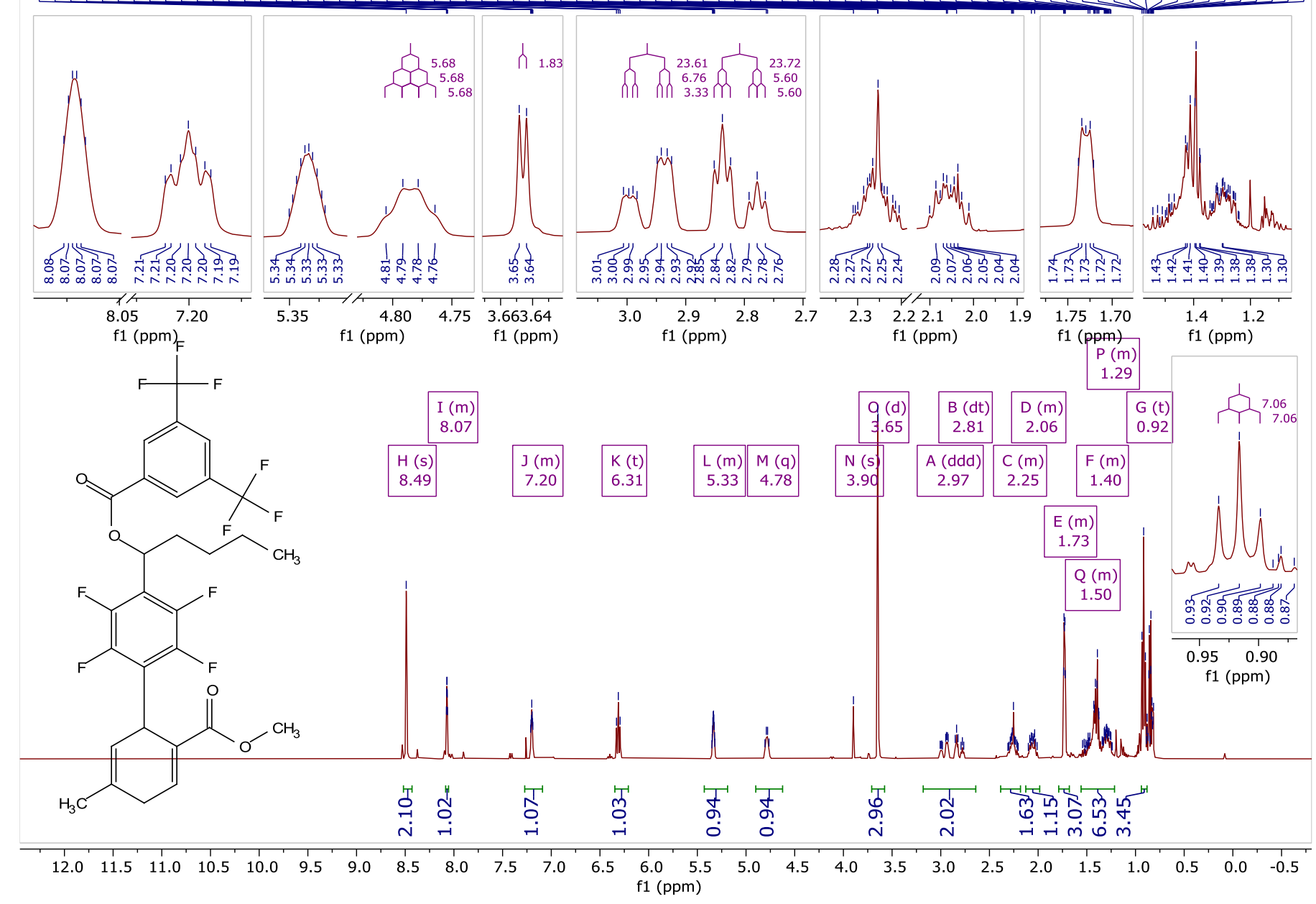
${ }^{13} \mathbf{C}\left\{{ }^{1} \mathrm{H}\right\}$ NMR $\left(\mathrm{CDCl}_{3}, 101 \mathrm{MHz}\right)$

(25)

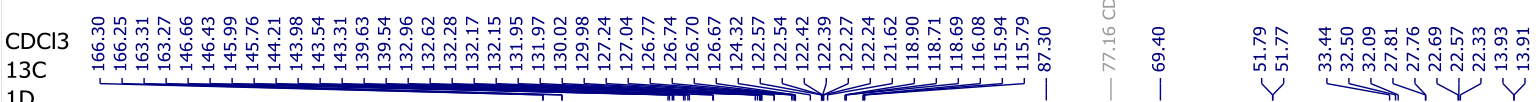

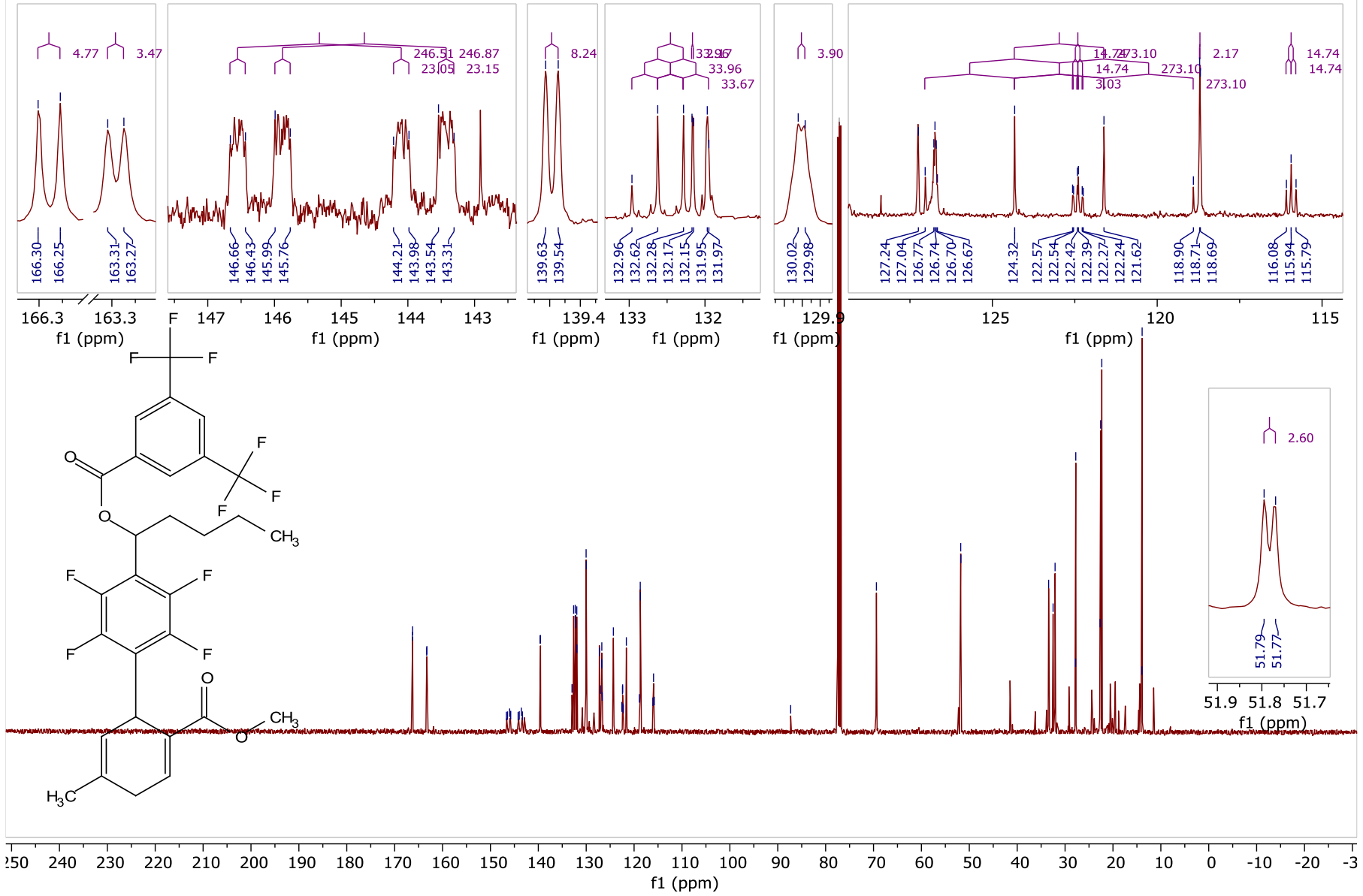


(26) Methyl 2',3',5',6'-tetrafluoro-5-methyl-4' -pentyl-1,4-dihydro-[1,1'-biphenyl]-2- carboxylate

${ }^{19}$ F NMR $\left(\mathrm{CDCl}_{3}, 376 \mathrm{MHz}\right)$

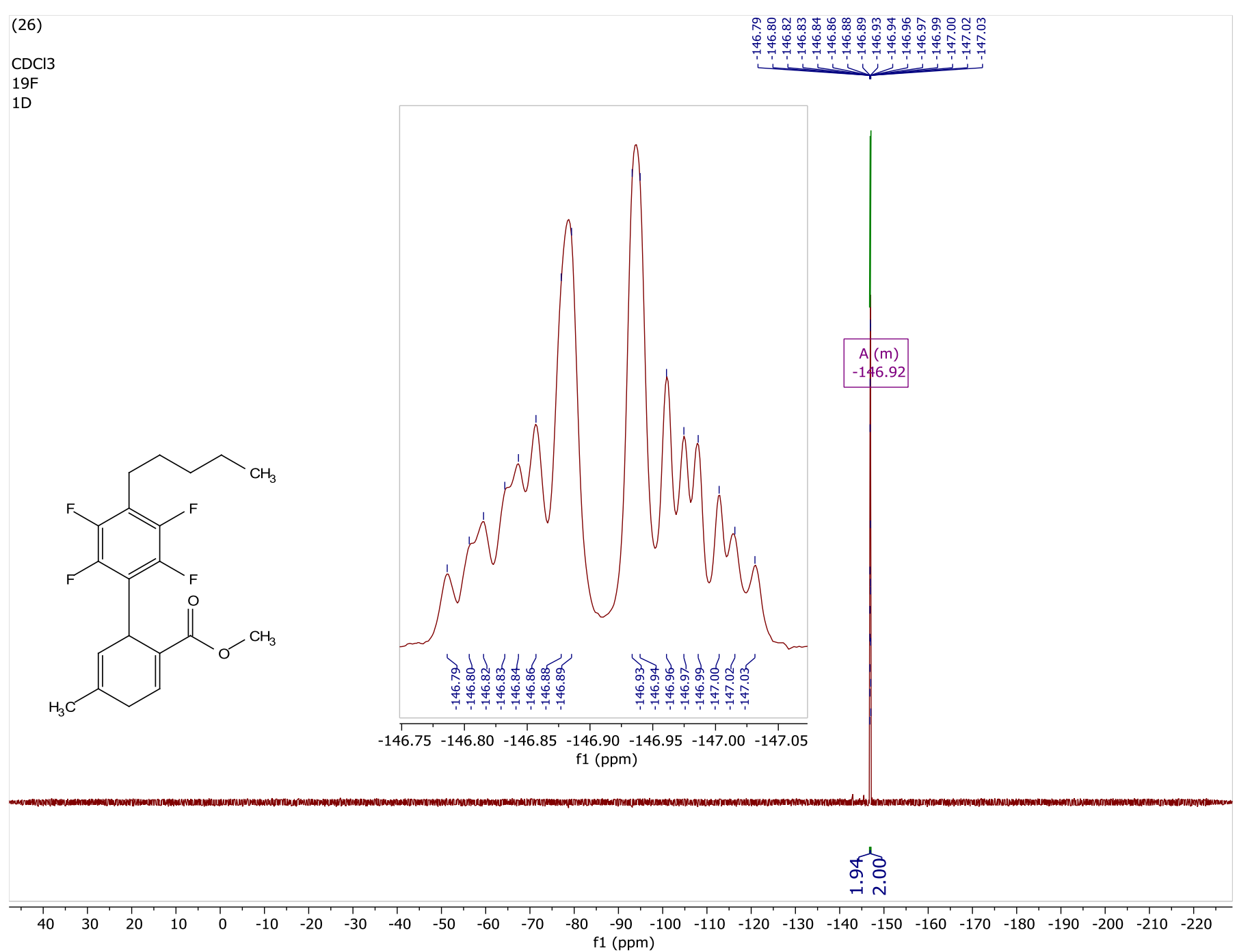


${ }^{1} \mathbf{H}$ NMR $\left(\mathrm{CDCl}_{3}, 400 \mathrm{MHz}\right)$

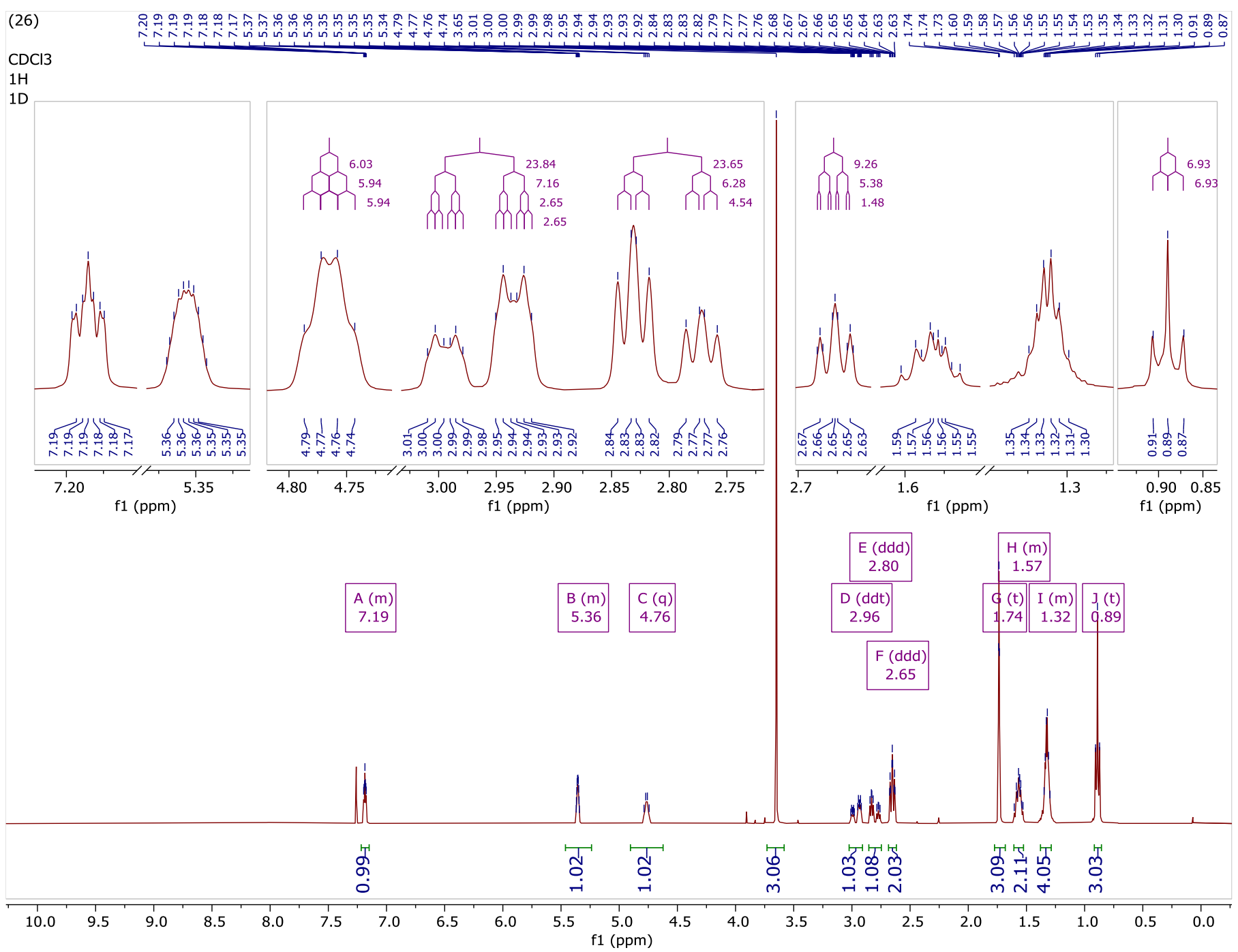


${ }^{13} \mathrm{C}\left\{{ }^{1} \mathrm{H}\right\}$ NMR $\left(\mathrm{CDCl}_{3}, 101 \mathrm{MHz}\right)$

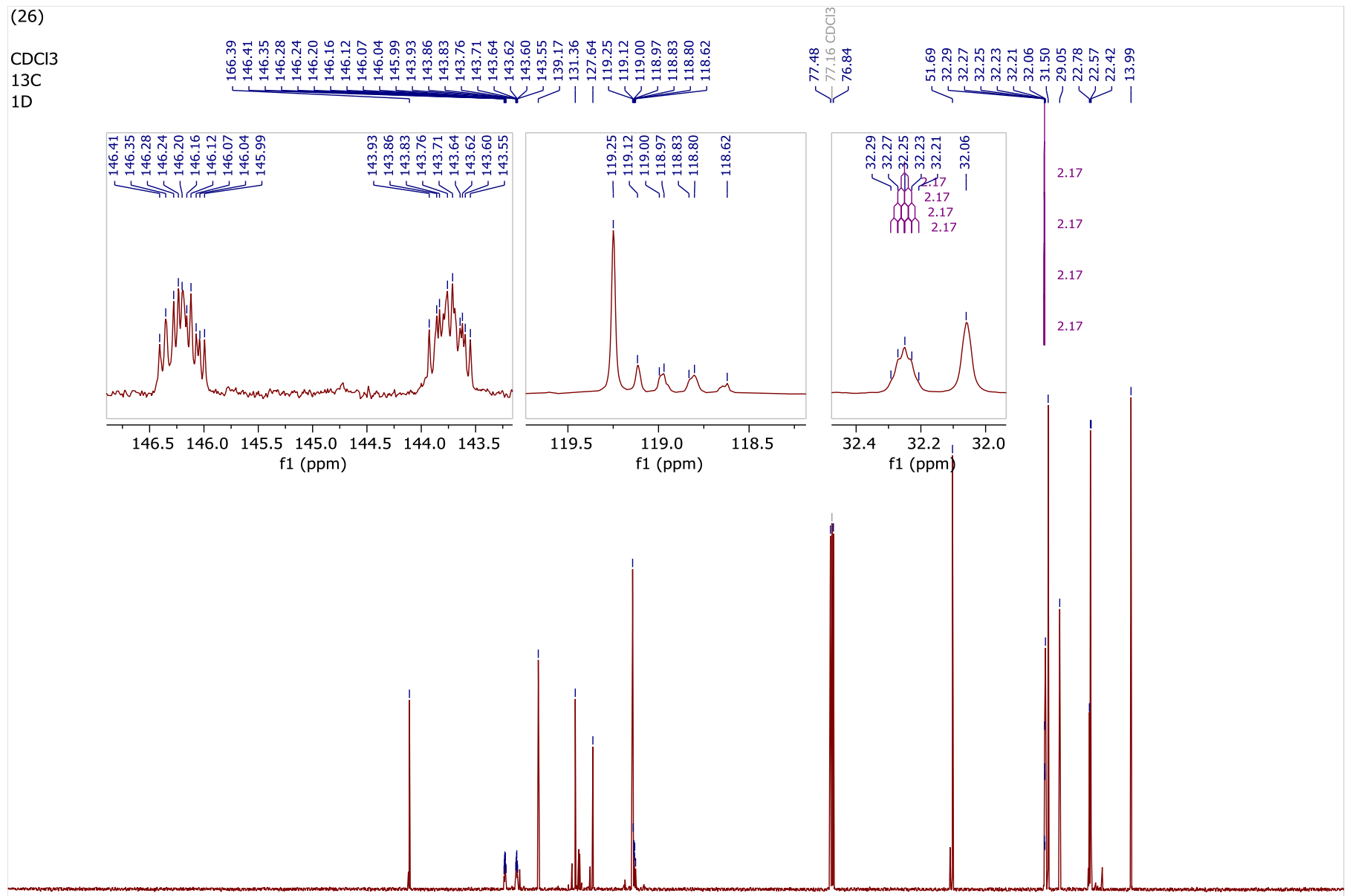
\begin{tabular}{lllllllllllllllllllllllllllllllllllllll}
\hline 50 & 240 & 230 & 220 & 210 & 200 & 190 & 180 & 170 & 160 & 150 & 140 & 130 & 120 & 110 & 100 & 90 & 80 & 70 & 60 & 50 & 40 & 30 & 20 & 10 & 0 & -10 & -20 & -3
\end{tabular} 
${ }^{1} \mathrm{H}-{ }^{13} \mathrm{C}$ HSQC

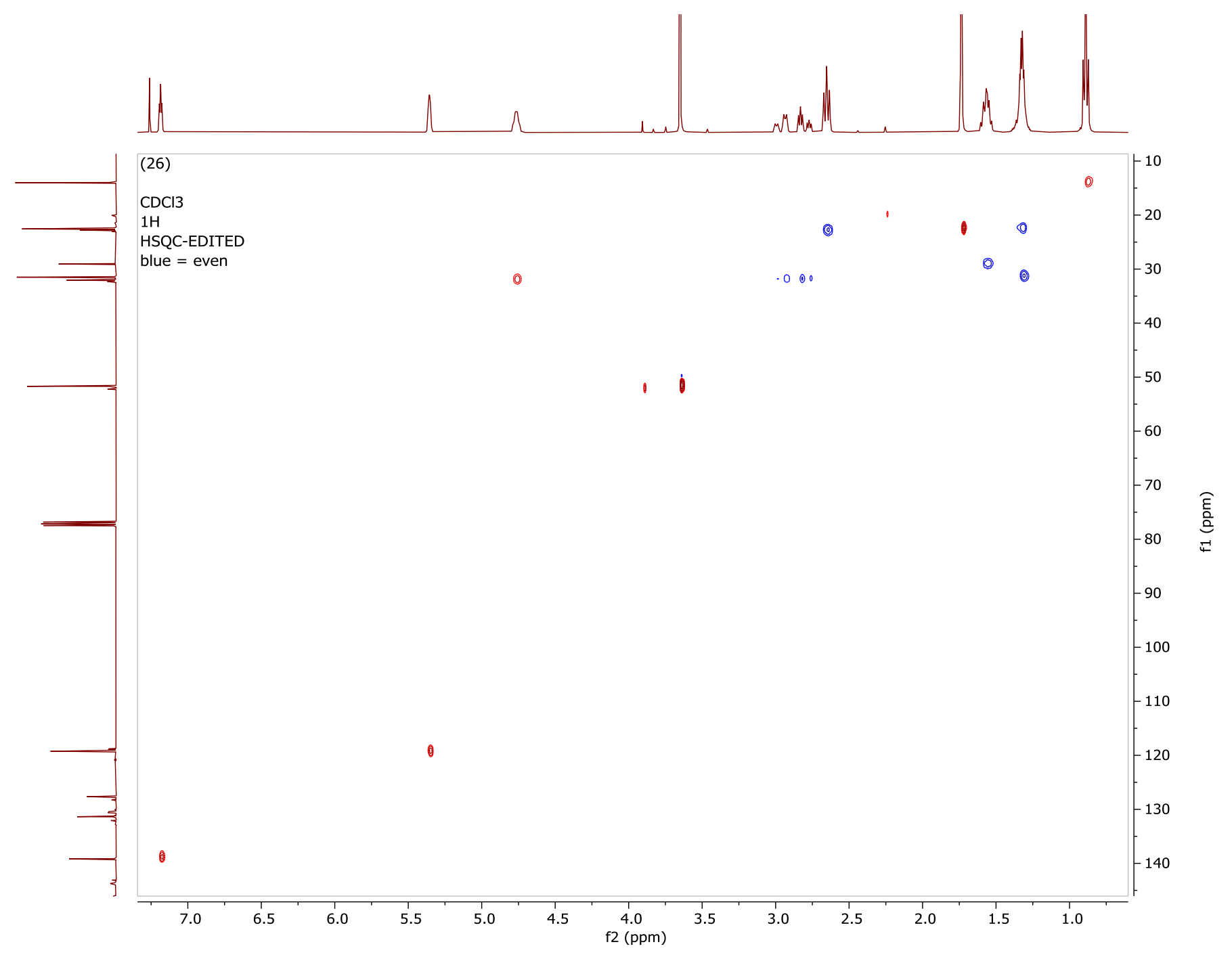


${ }^{1} \mathrm{H}-{ }^{1} \mathrm{H}$ COSY

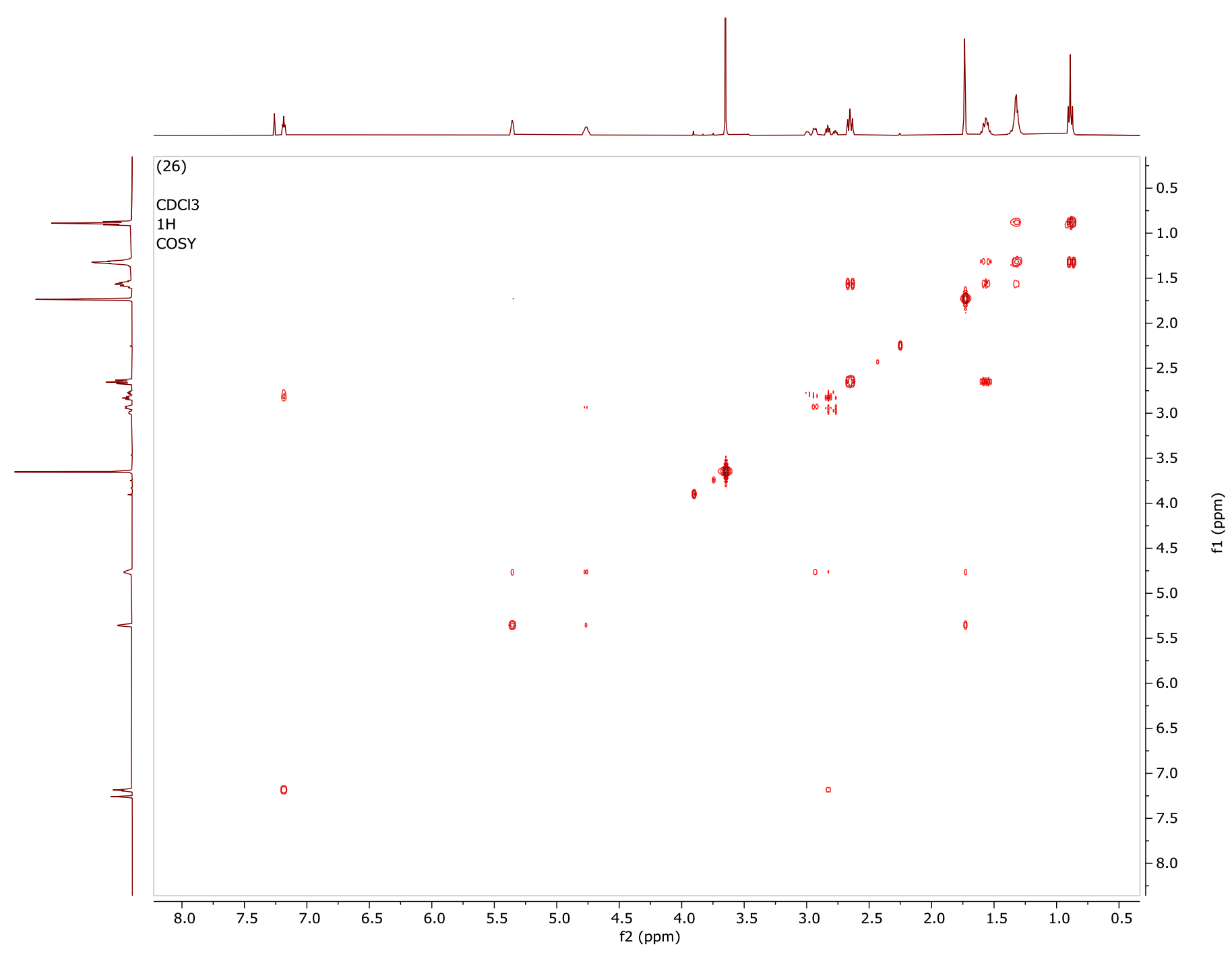


(29) Methyl 2',3',5',6'-tetrafluoro-5-methyl-4' -pentyl-1,2,3,4-tetrahydro-[1,1'-biphenyl]- 2-carboxylate

${ }^{19} \mathbf{F}$ NMR $\left(\mathrm{CDCl}_{3}, 376 \mathrm{MHz}\right)$

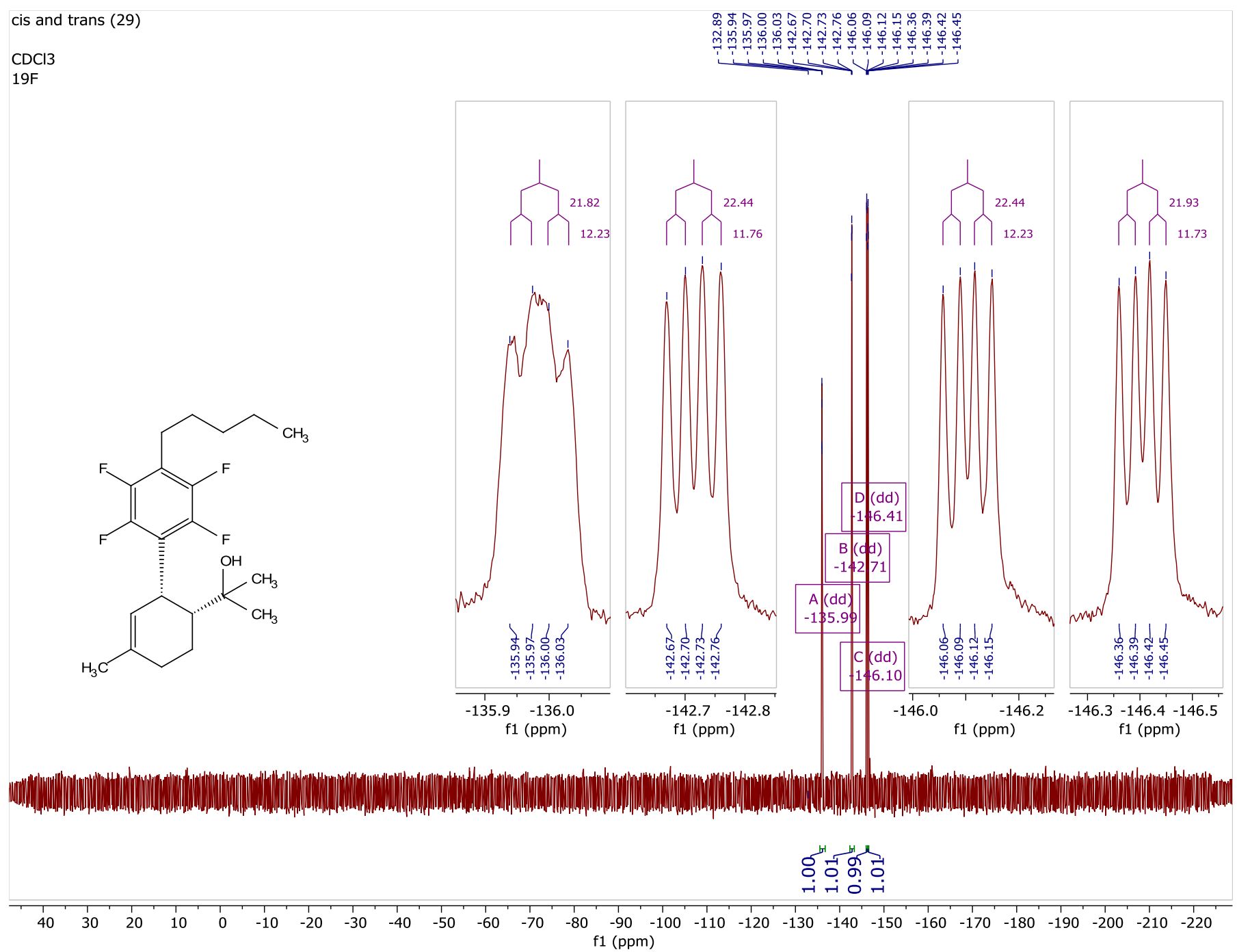


${ }^{1} \mathbf{H}$ NMR $\left(\mathrm{CDCl}_{3}, 400 \mathrm{MHz}\right)$

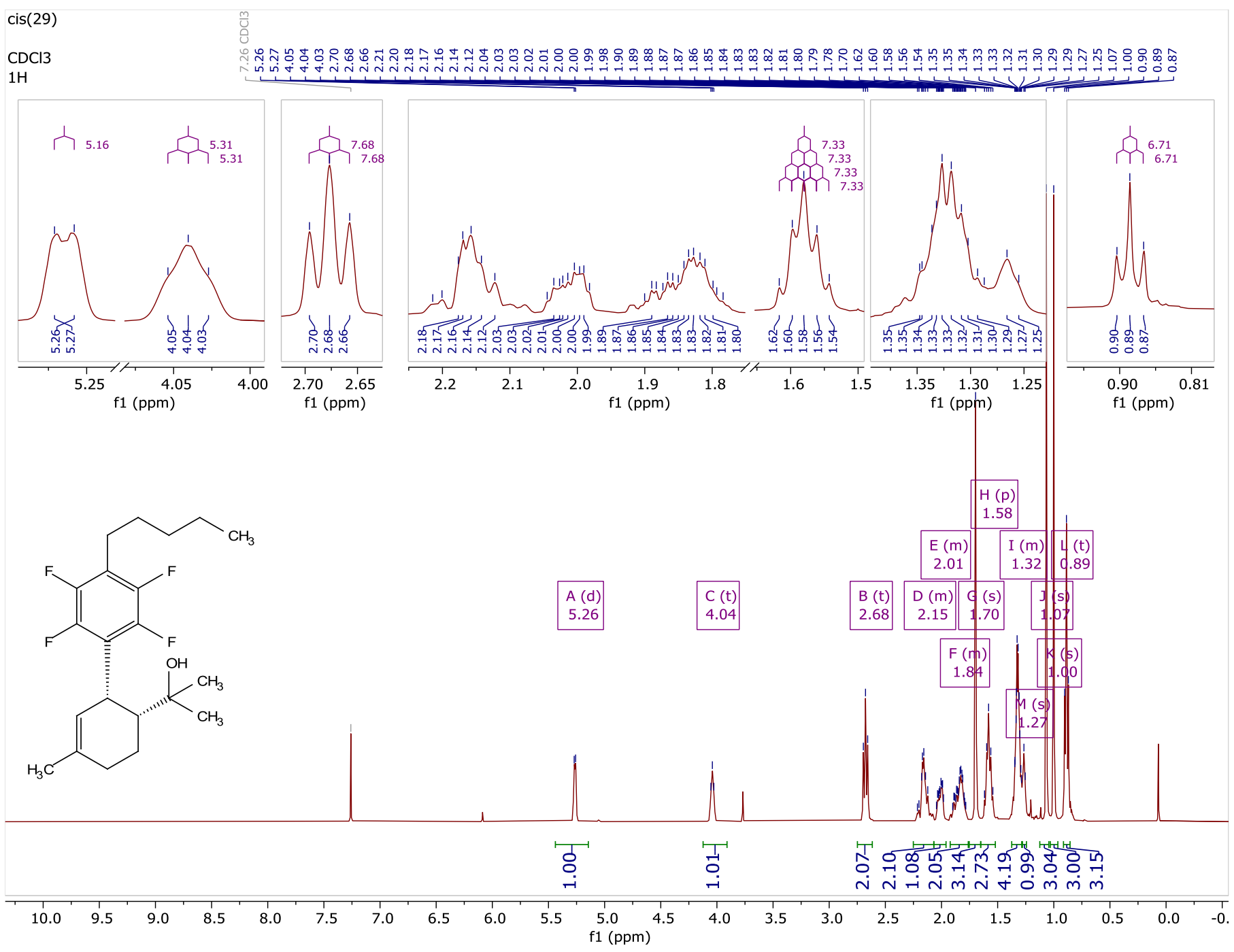


${ }^{13} \mathbf{C}\left\{{ }^{1} \mathbf{H}\right\}$ NMR $\left(\mathrm{CDCl}_{3}, 101 \mathrm{MHz}\right)$

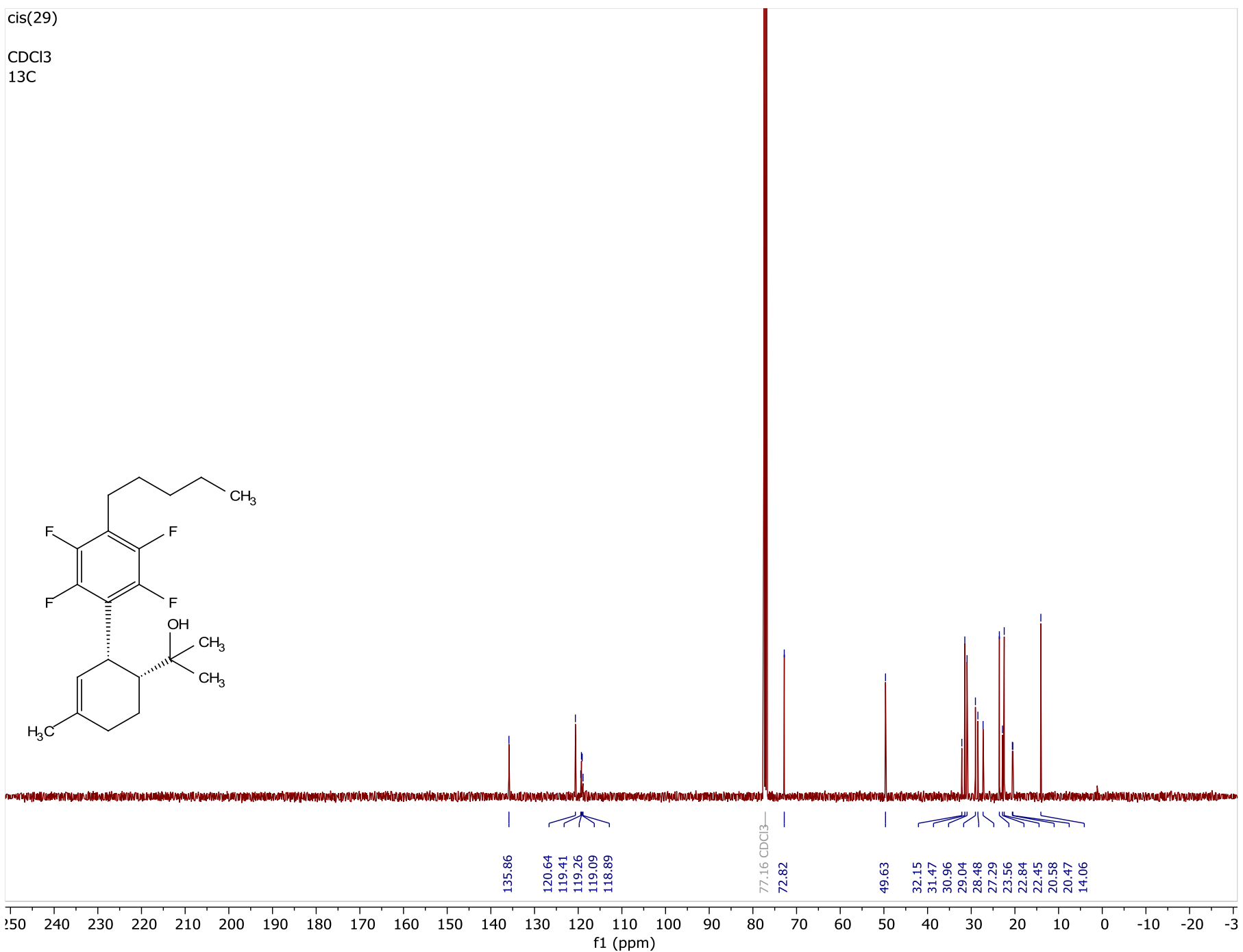


${ }^{19}$ F NMR $\left(\mathrm{CDCl}_{3}, 376 \mathrm{MHz}\right)$

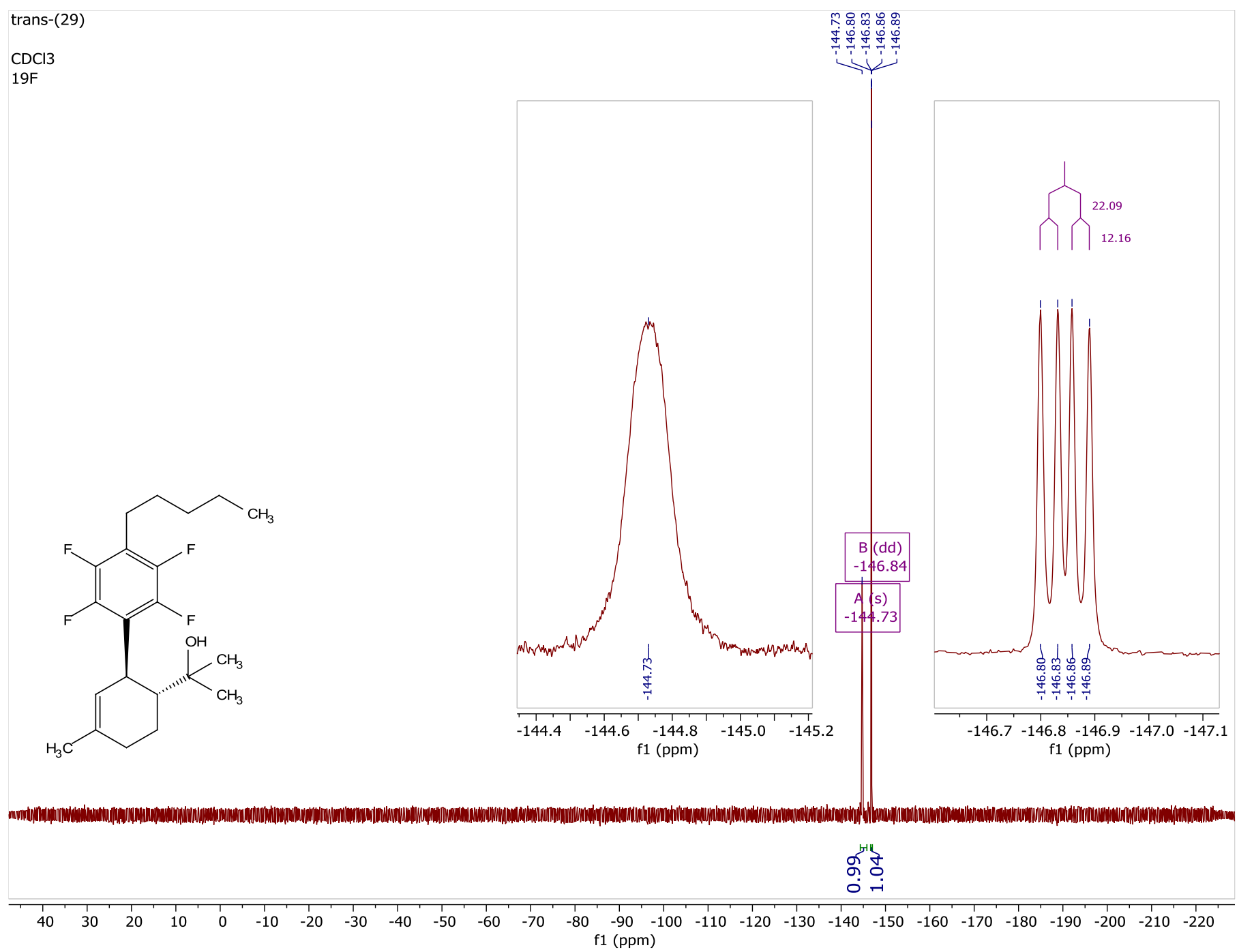


${ }^{1} \mathbf{H}$ NMR $\left(\mathrm{CDCl}_{3}, 400 \mathrm{MHz}\right)$

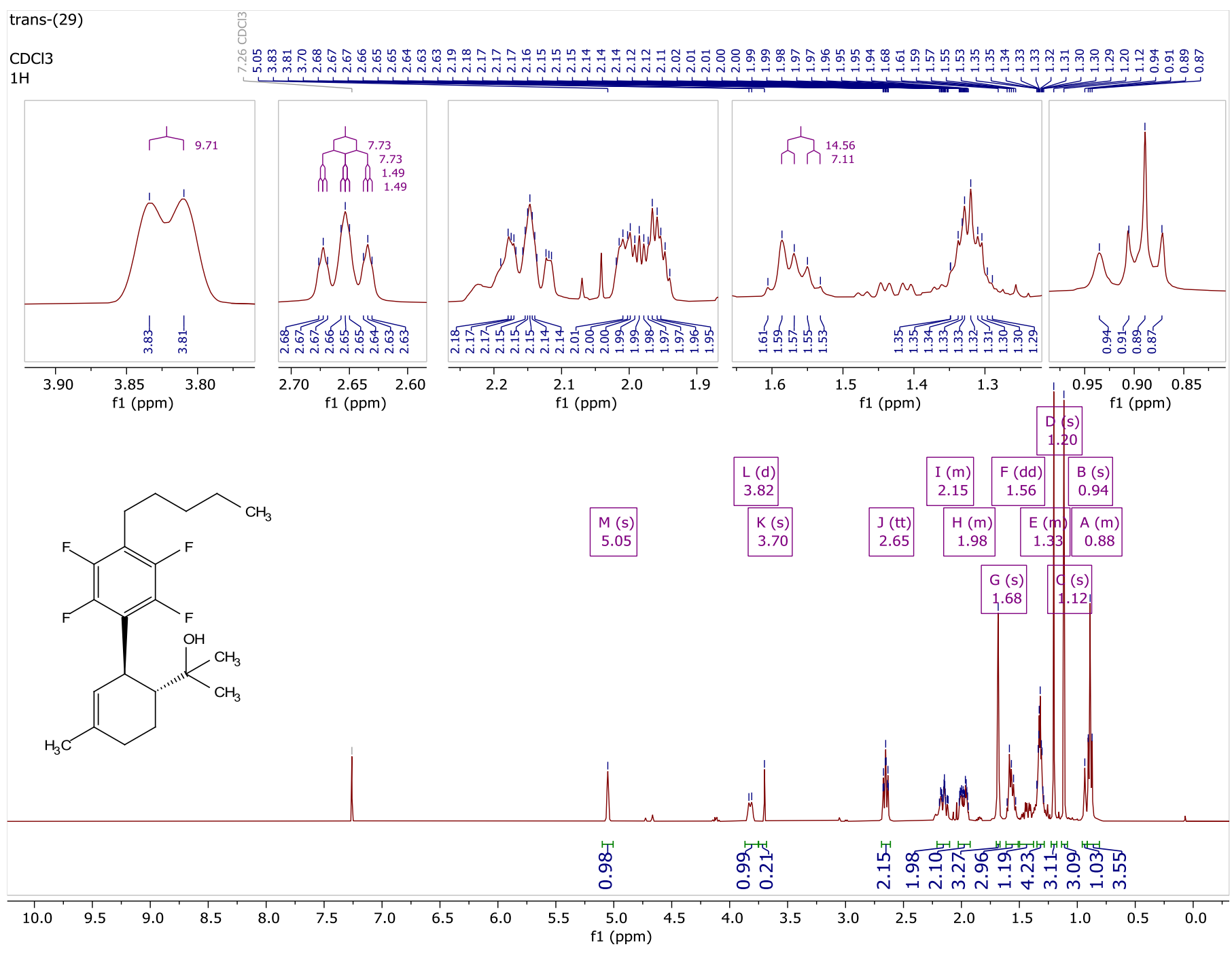


${ }^{13} \mathbf{C}\left\{{ }^{1} \mathbf{H}\right\}$ NMR $\left(\mathrm{CDCl}_{3}, 101 \mathrm{MHz}\right)$

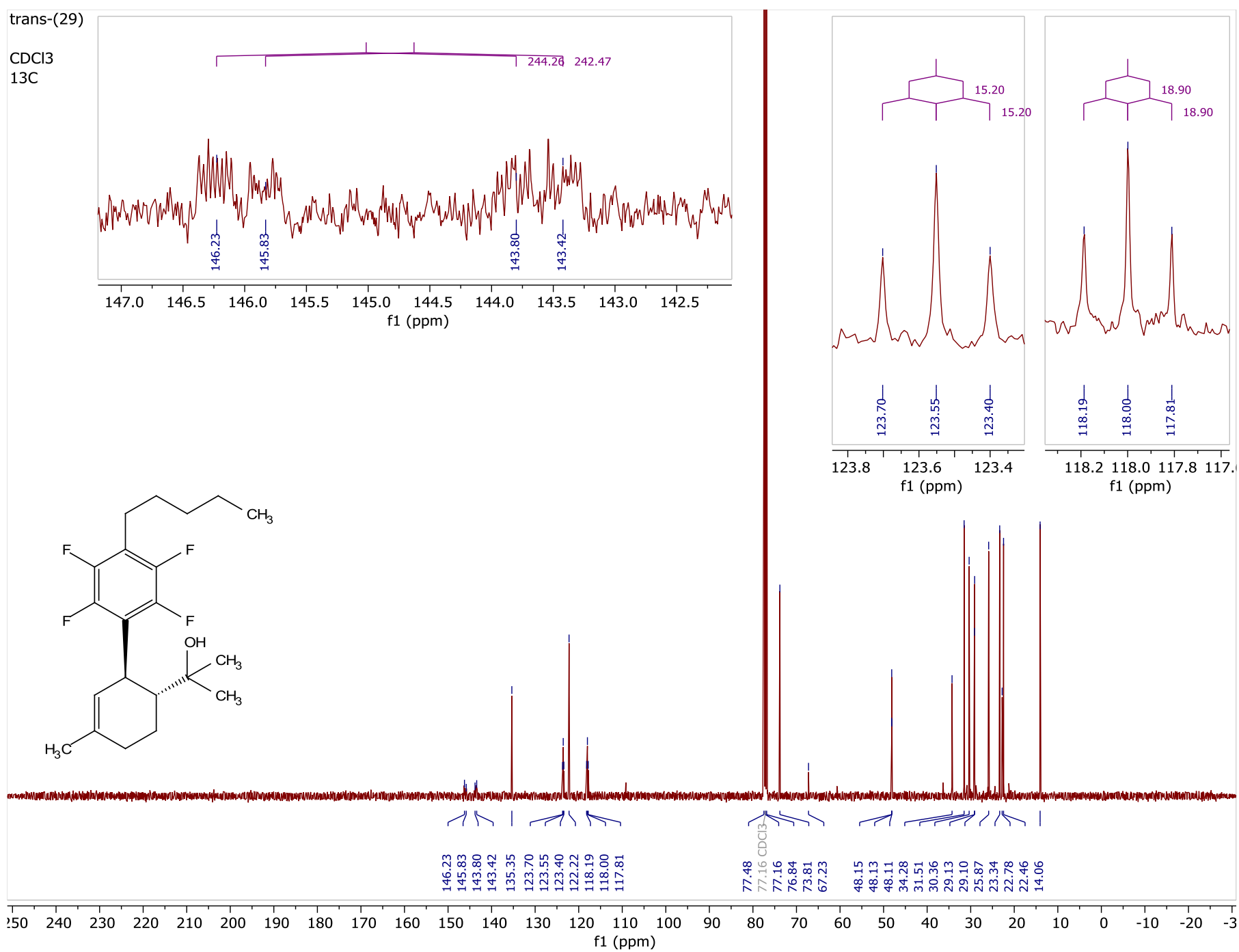


${ }^{1} \mathrm{H}-{ }^{13} \mathrm{C}$ HSQC

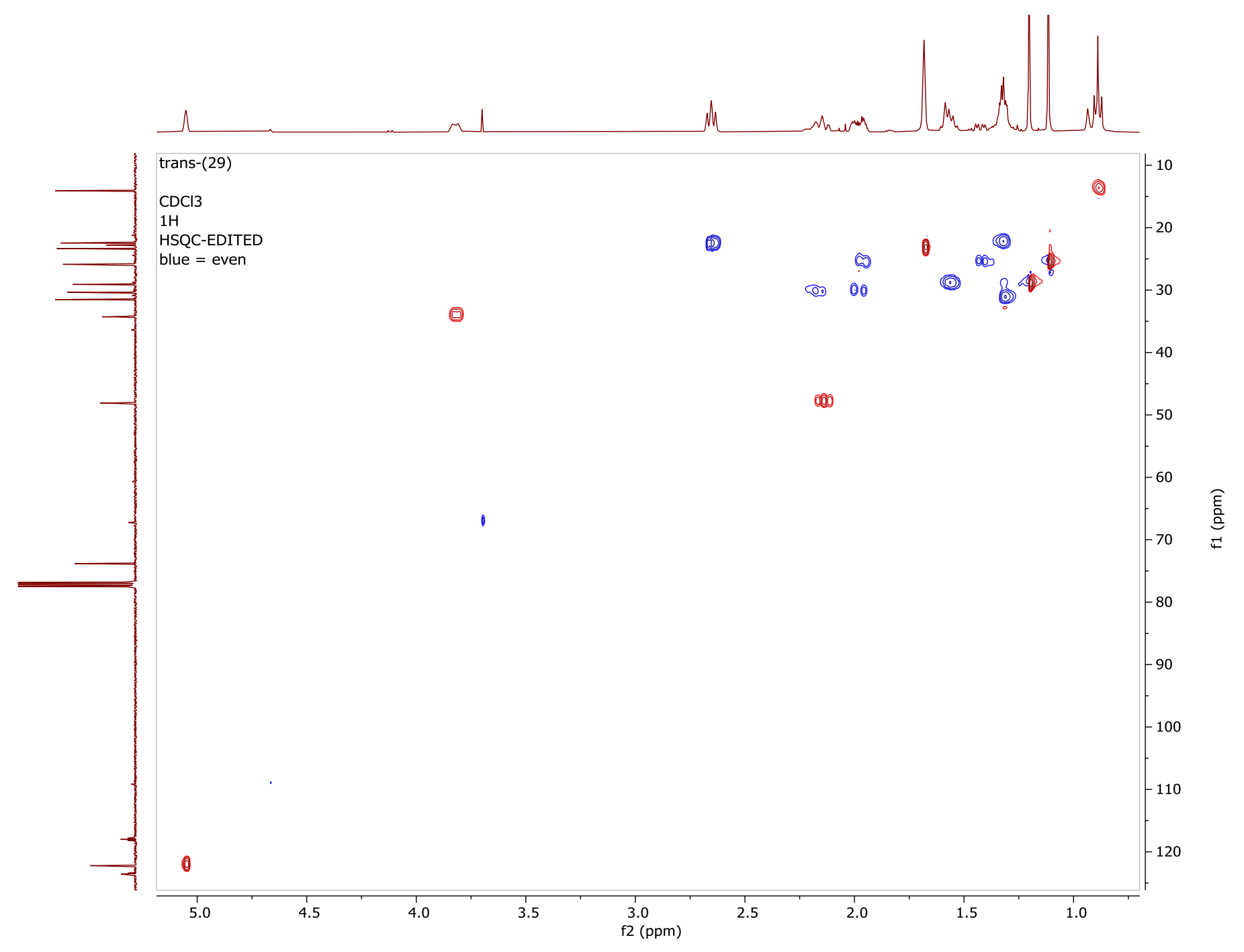


${ }^{1} \mathrm{H}-{ }^{1} \mathrm{H}$ COSY

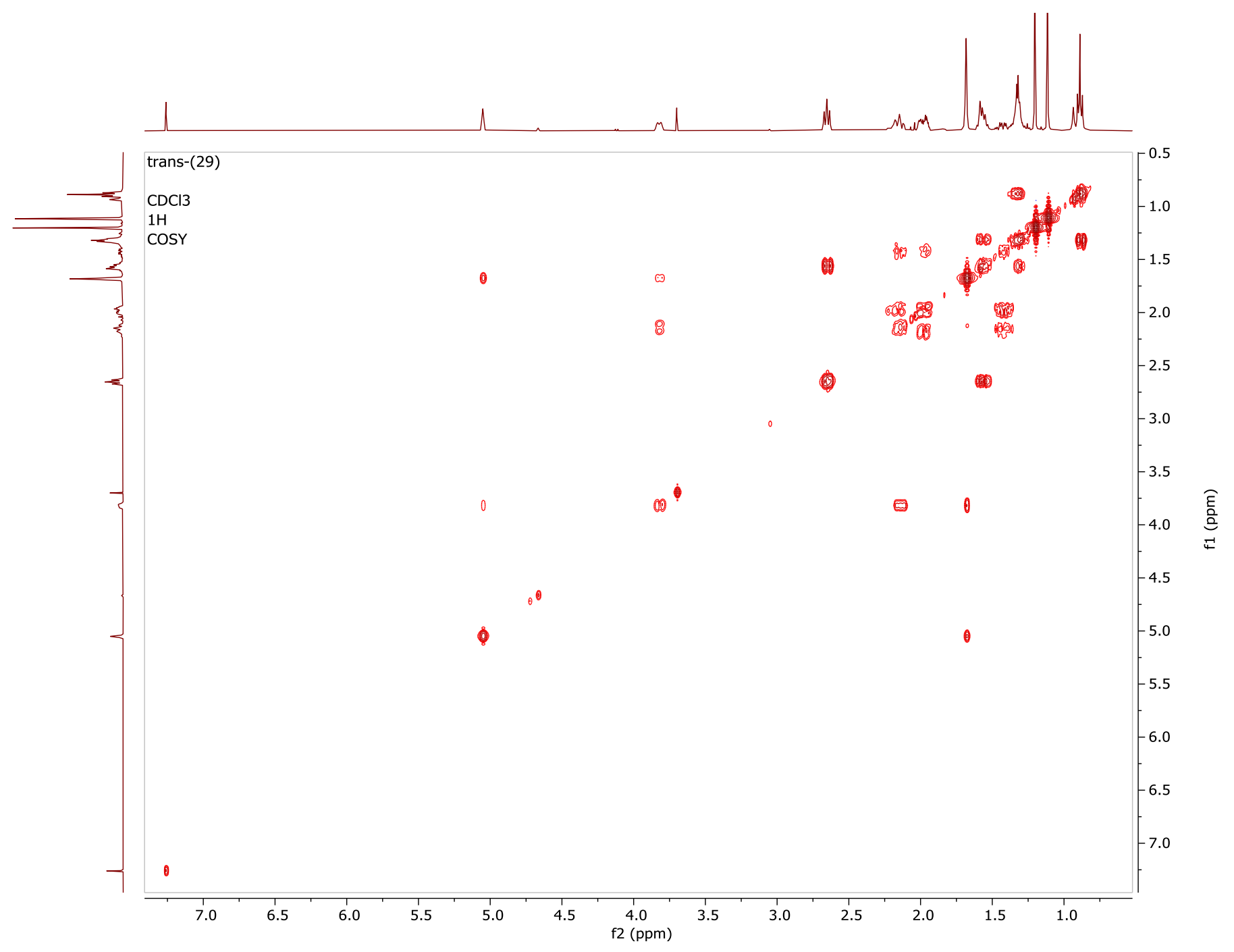


(30) 1,2,4-Trifluoro-6,6,9-trimethyl-3-pentyl-6a,7,8,10a-tetrahydro-6Hbenzo[c]chromene (1-deoxy-1,2,4-trifluoro-THC)

${ }^{19} \mathbf{F}$ NMR $\left(\mathrm{CDCl}_{3}, 376 \mathrm{MHz}\right)$

(30)
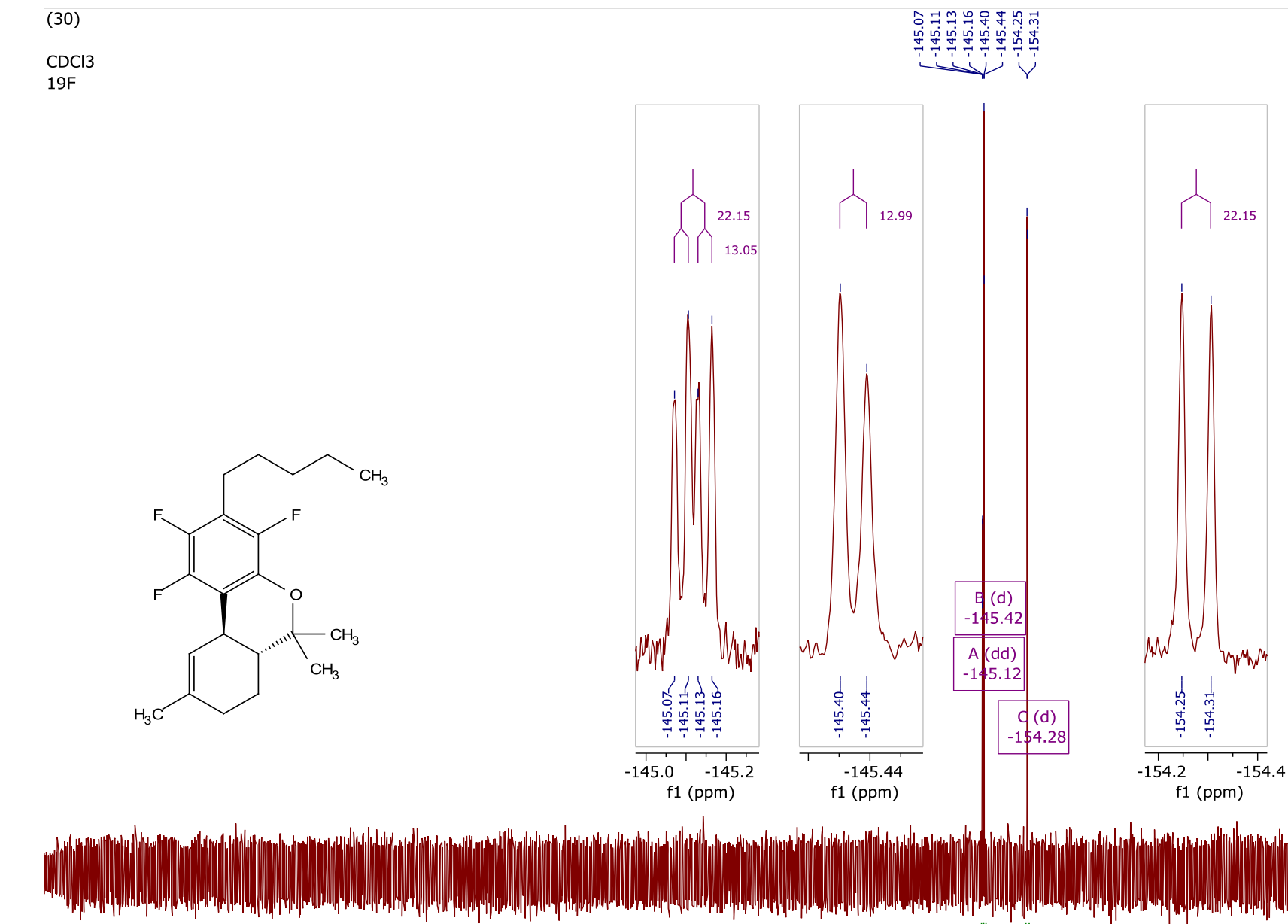

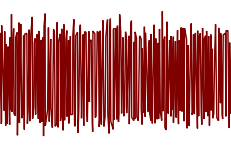

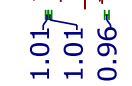

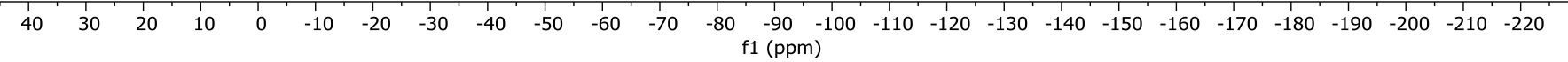


'H NMR (CDCl, $400 \mathrm{MHz})$

(30)

$\mathrm{CDCl} 3$
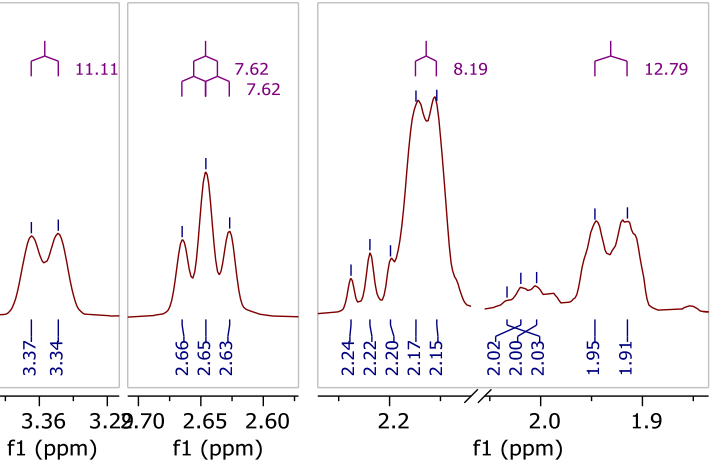

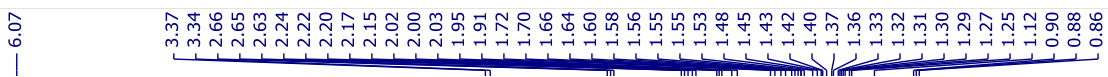

f1 $(\mathrm{ppm}) \quad \mathrm{f1}(\mathrm{ppm})$
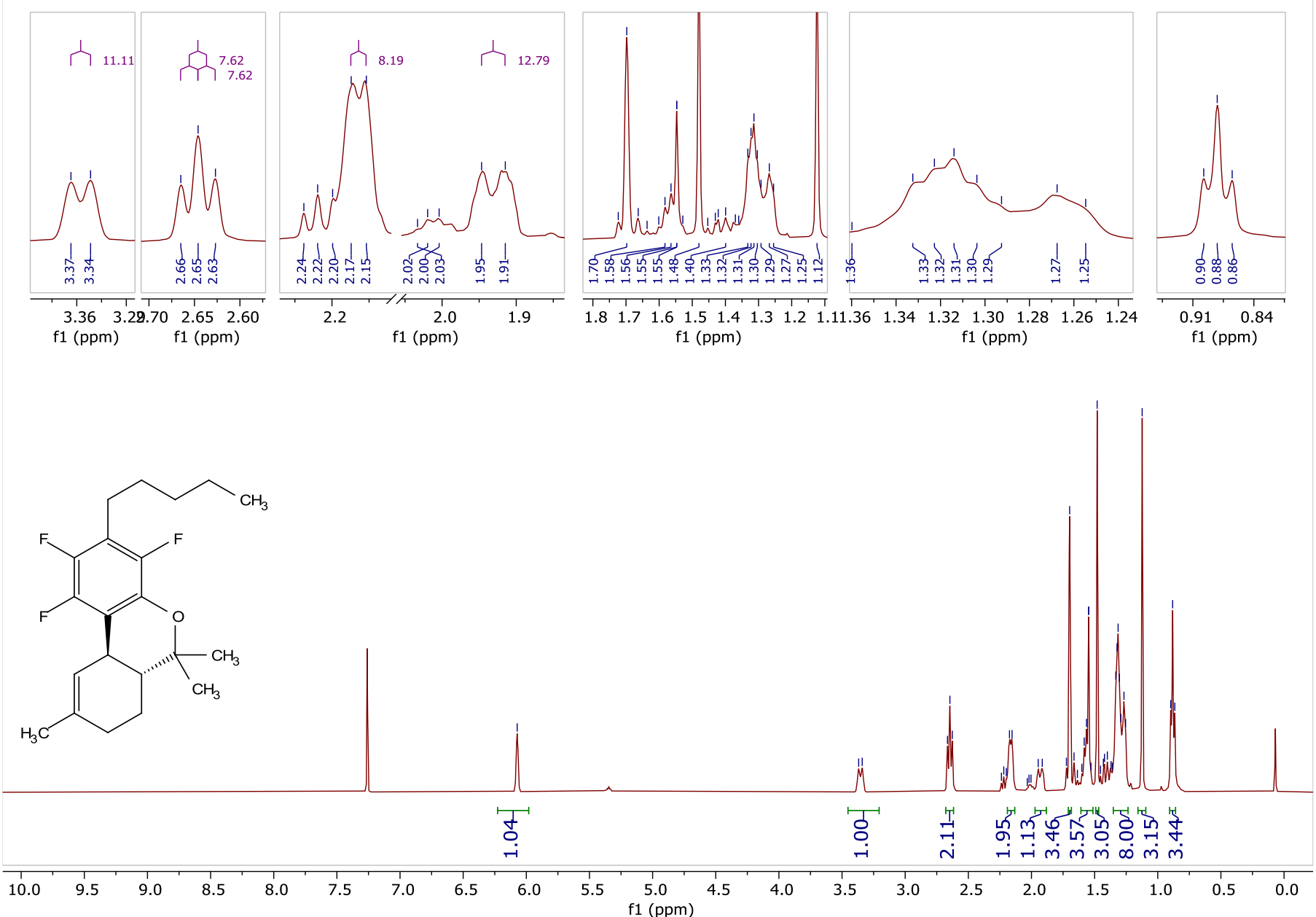

f1 (ppm)

f1 (ppm)

$0.91(\mathrm{ppm})$
$\mathrm{f} 1(\mathrm{ph})$ 
${ }^{13} \mathrm{C}\left\{{ }^{1} \mathrm{H}\right\}$ NMR $\left(\mathrm{CDCl}_{3}, 101 \mathrm{MHz}\right)$

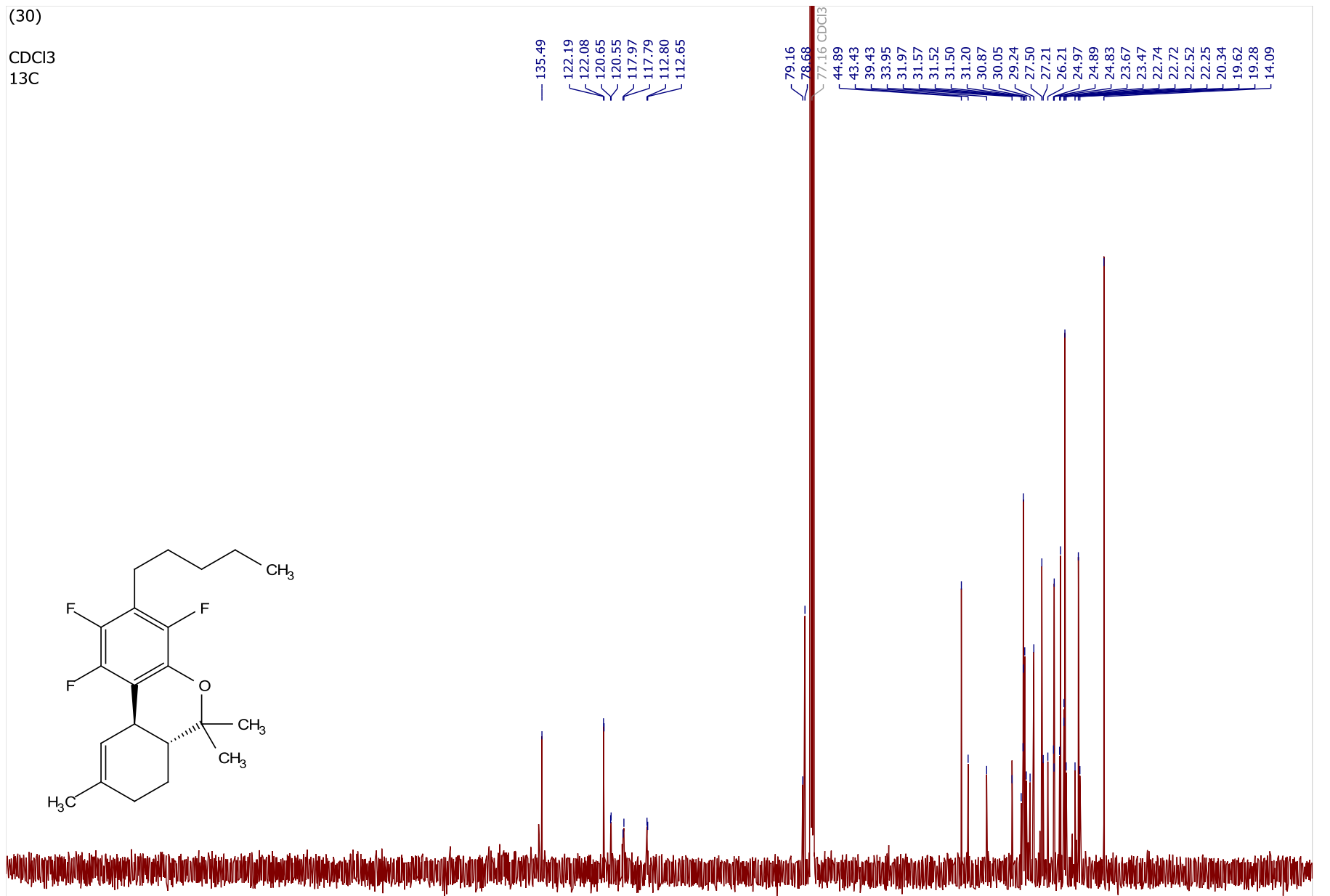

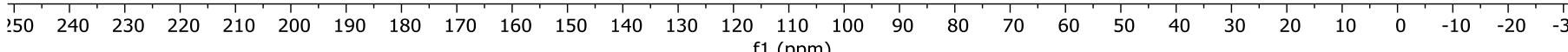


${ }^{1} \mathrm{H}-{ }^{13} \mathrm{C}$ HSQC

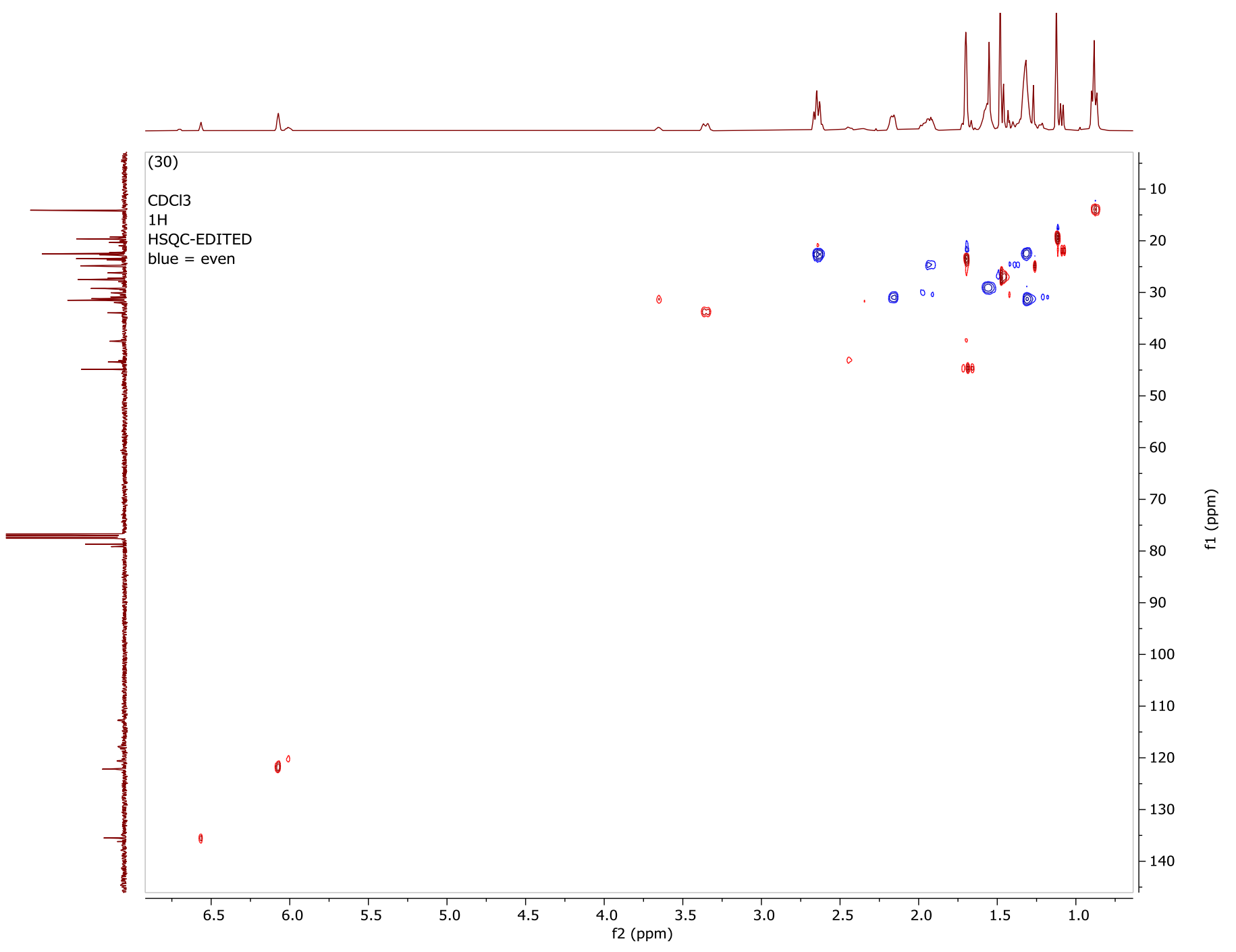


${ }^{1} \mathrm{H}-{ }^{1} \mathrm{H} \cos Y$

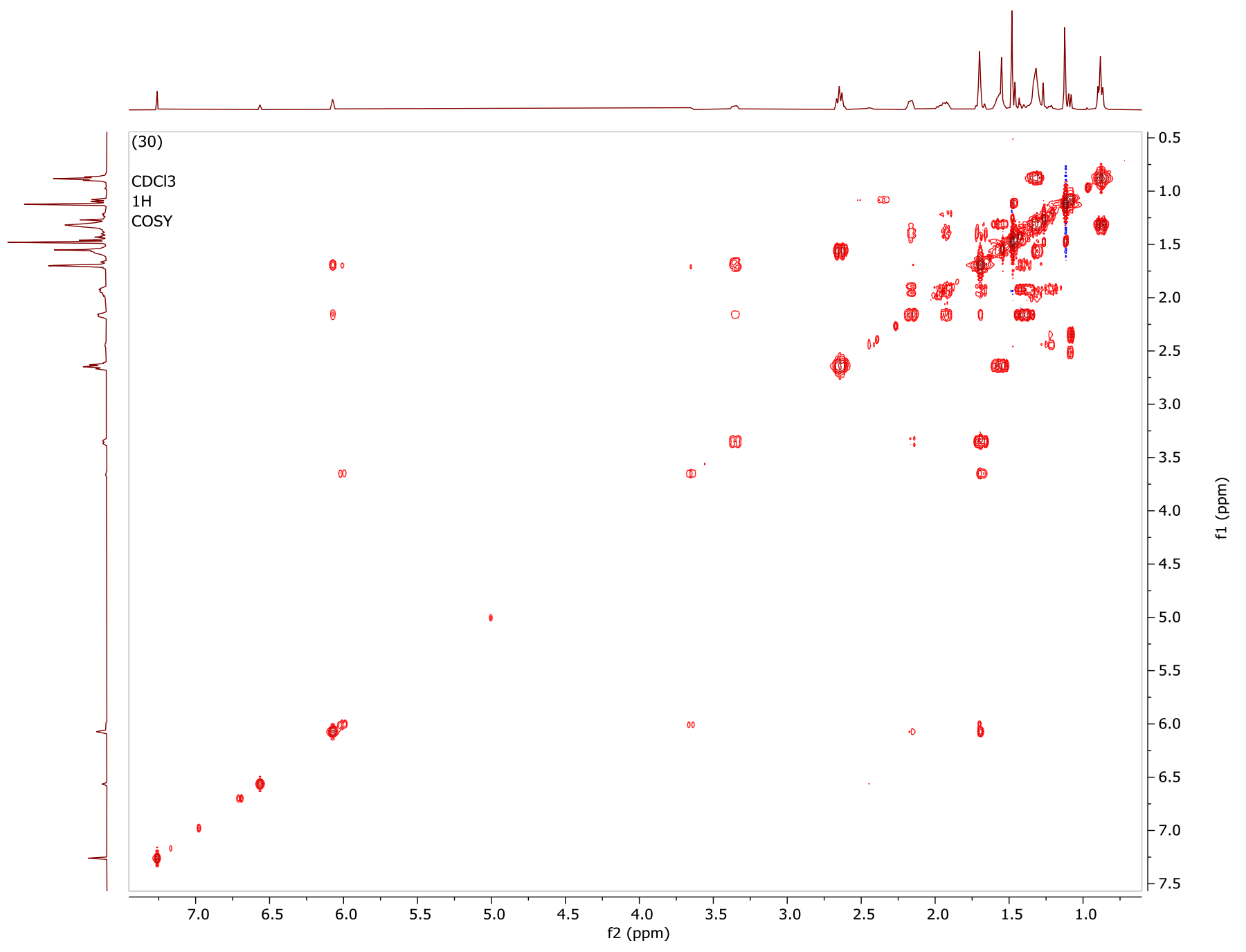




\section{${ }^{1} \mathrm{H}-{ }^{1} \mathrm{H}$ NOESY}

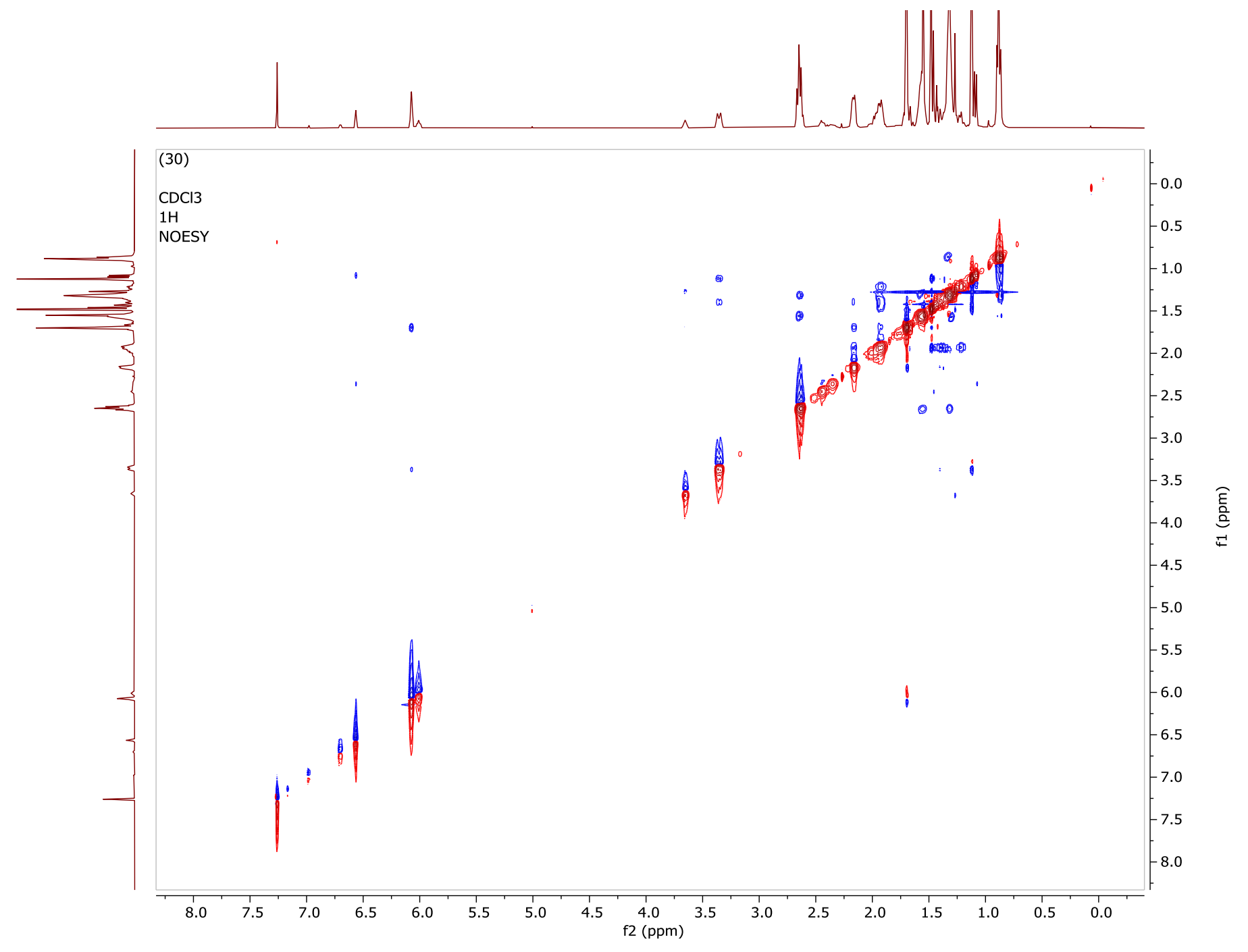


${ }^{19}$ F NMR $\left(\mathrm{CDCl}_{3}, 376 \mathrm{MHz}\right)$

(30) cis and trans

$\mathrm{CDCl} 3$

$19 \mathrm{~F}$

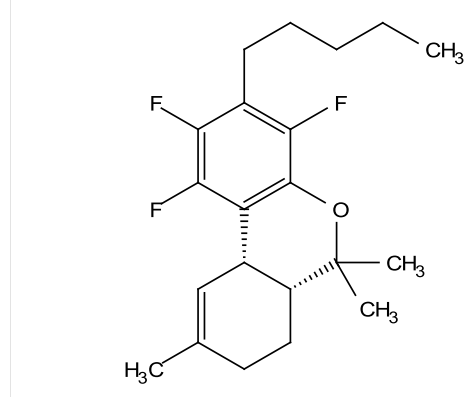

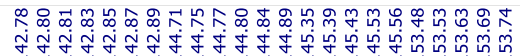

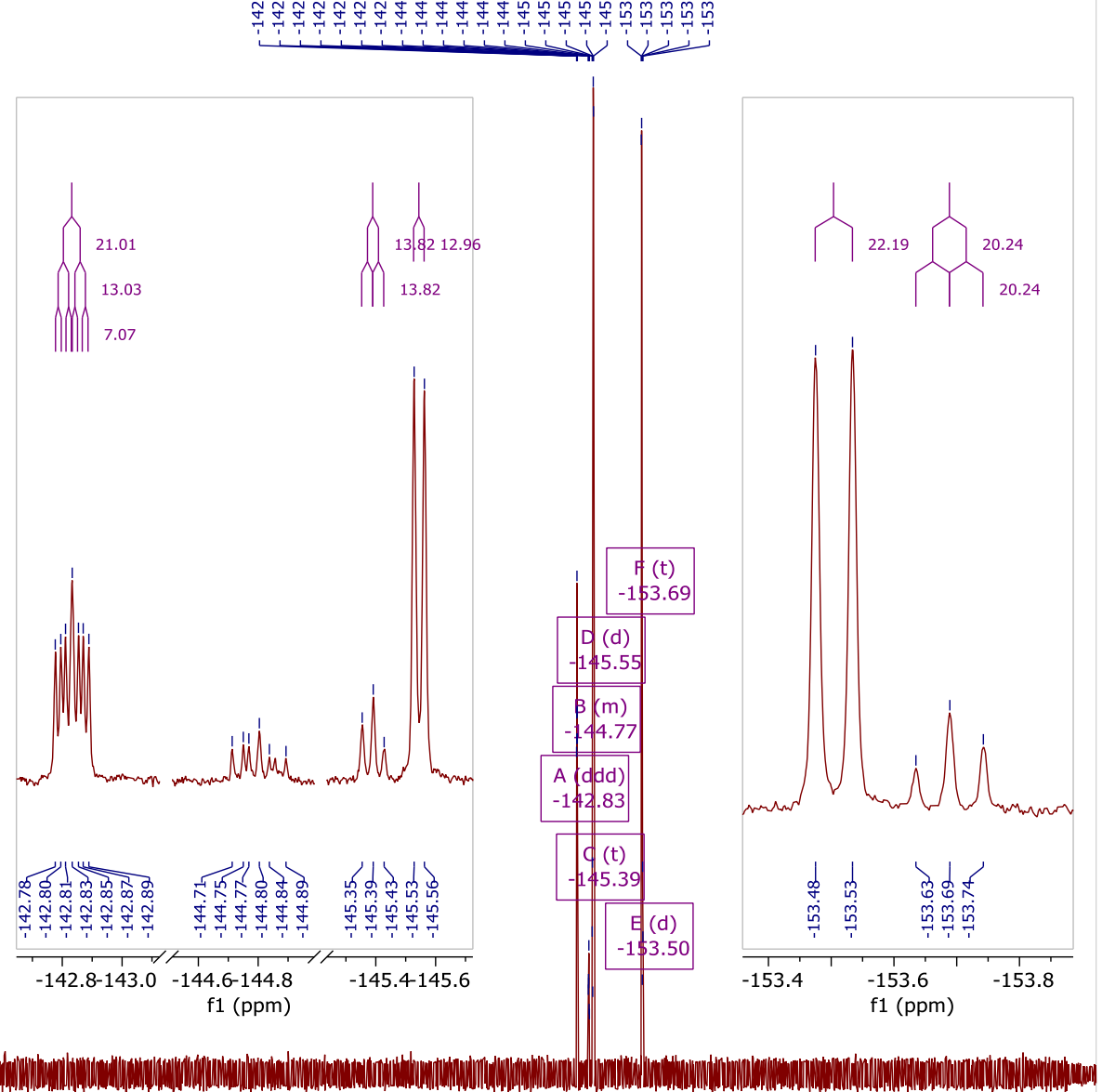

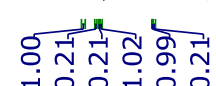

$\begin{array}{llllllllllllllllllllllllllllllllllll}40 & 30 & 20 & 10 & 0 & -10 & -20 & -30 & -40 & -50 & -60 & -70 & -80 & -90 & -100 & -110 & -120 & -130 & -140 & -150 & -160 & -170 & -180 & -190 & -200 & -210 & -220\end{array}$ 
${ }^{1} \mathbf{H}$ NMR $\left(\mathrm{CDCl}_{3}, 400 \mathrm{MHz}\right)$

(30) cis and trans

$\mathrm{CDCl} 3$

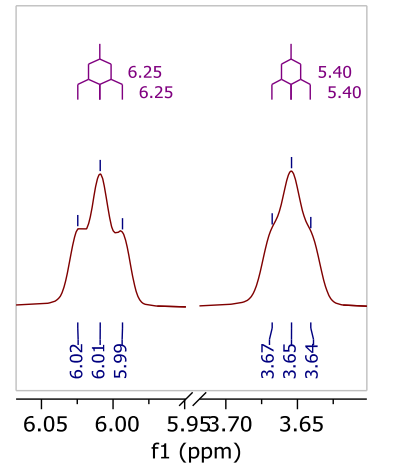

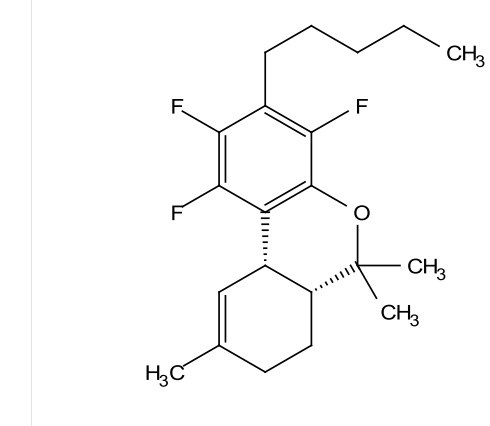

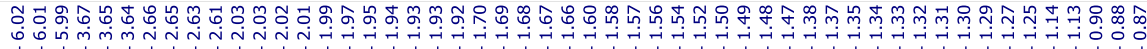

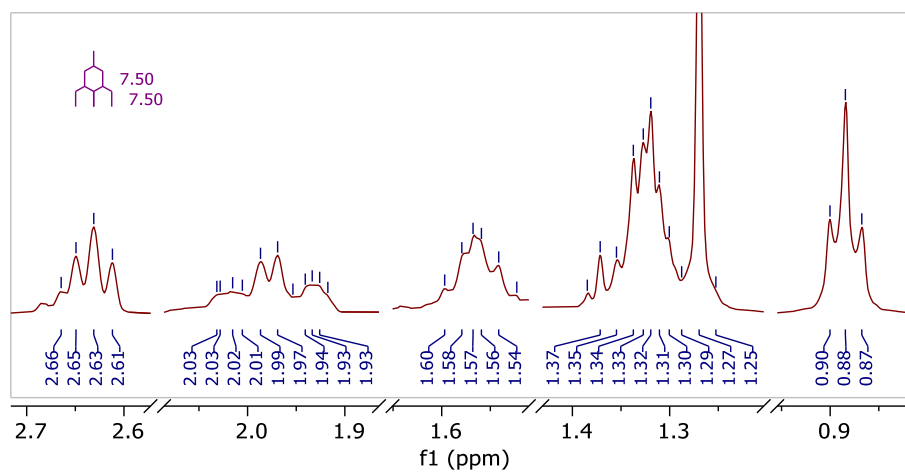

$1(\mathrm{ppm})$
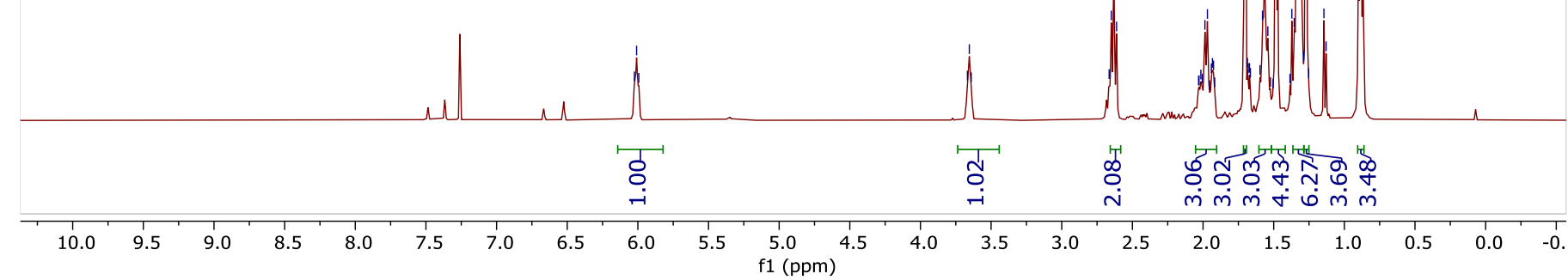


\section{Computational}

Calculations were conducted using Gaussian 09 RevE.01 on the HPC-cluster Athene at University of Regensburg or using Gaussian 09 , Revision C.01 on Cowboy or Pete supercomputing clusters at the Oklahoma State University. Geometries at or near minima as determined by frequency calculation.

\section{Computational NMR spectra}

Computational NMR Spectra General Procedure: structures were built in Chem3D ver 17.1.0.105. Low energy conformational search was performed using stochastic conformational analysis was performed (MMF94), varying by 3 nm, generating 10 structures which were minimized in 500 steps. The low energy conformer was selected, exported as a PDB, and subjected to minimization in Gaussian. Methods same as described in Bally \& Rablen. ${ }^{3}$ Methyl signals were averaged following completion of calculation. Spectra were analyzed in MestreNova.<smiles>CC(=O)C1=CC=C(C)CC1c1c(F)c(F)nc(F)c1F</smiles>

Diene Structure A

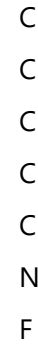

C
C
C
C
C
N
F

C

c

C

$\mathrm{F}$

$\mathrm{F}$

C

C

O

C

C

C

C

$\mathrm{H}$

$\mathrm{H}$

$\mathrm{H}$

$\mathrm{H}$

$\mathrm{H}$

$\mathrm{H}$

$\mathrm{H}$

$\mathrm{H}$

$\mathrm{H}$

$\mathrm{H}$

H
2.437508

$$
2.12956
$$

3.134767

4.412496

4.612408

3.650092

1.459861

5.464506

2.811558

5.044653

2.380228

0.867334

5.846329

3.487286

3.094526

1.271948

4.786576

4.140228

2.182532

5.691364

1.732254

1.599968

2.318611

5.941319

2.785889

4.335194

3.360729

6.074526

6.546521

5.150945

3.626284
$3.506468 \quad 1.052895$

$2.156784 \quad 0.89753$

$1.244149 \quad 0.563841$

$1.788777 \quad 0.417087$

$3.155504 \quad 0.596863$

$3.996418 \quad 0.906482$

$4.357797 \quad 1.372831$

$1.009242 \quad 0.113967$

$-0.23701 \quad 0.390186$

$-2.00826-0.25495$

$-0.64374-3.53015$

$1.749309 \quad 1.086835$

$3.6491 \quad 0.451908$

$-1.141 \quad 1.460206$

$-0.71308-1.03169$

$0.558514-1.78869$

$-1.80011 \quad 1.051536$

$-1.535-1.28613$

$-0.20868 \quad-2.08474$

$-2.2674 \quad 2.152091$

$-0.30249 \quad 0.537386$

$-0.18114-4.13689$

$-1.73324-3.63271$

$-2.54158 \quad-0.56049$

$-1.95024 \quad 1.718143$

$-1.85604-2.30613$

$-0.33121-3.90801$

$\begin{array}{ll}-1.4134 & 2.72781\end{array}$

$-2.83005 \quad 1.765952$

$-2.90561 \quad 2.865443$

$-0.57378 \quad 2.388598$

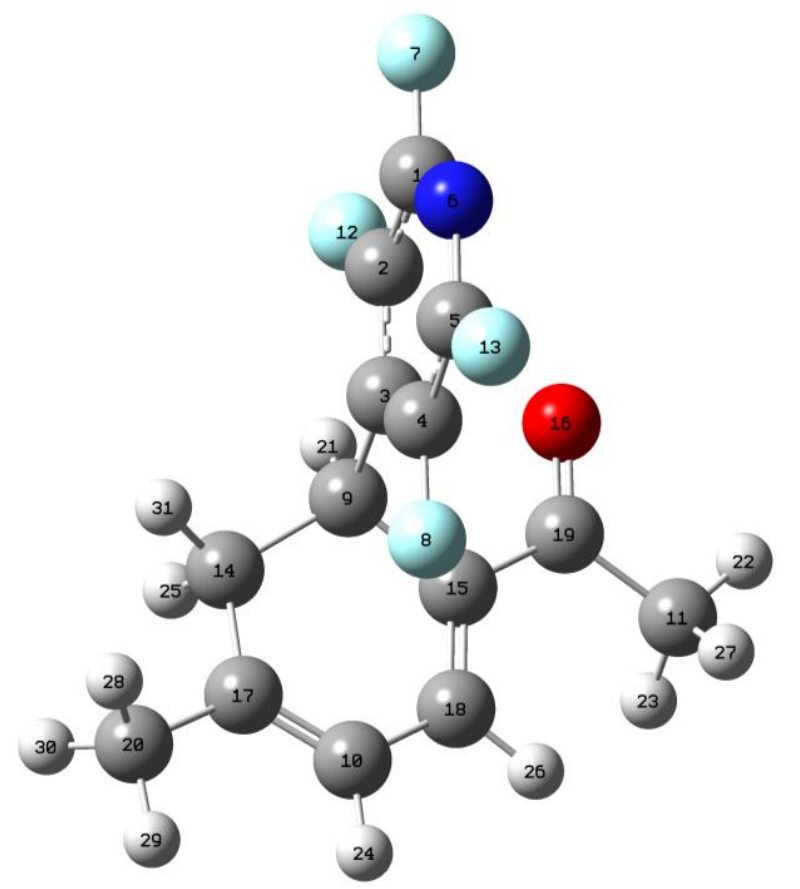




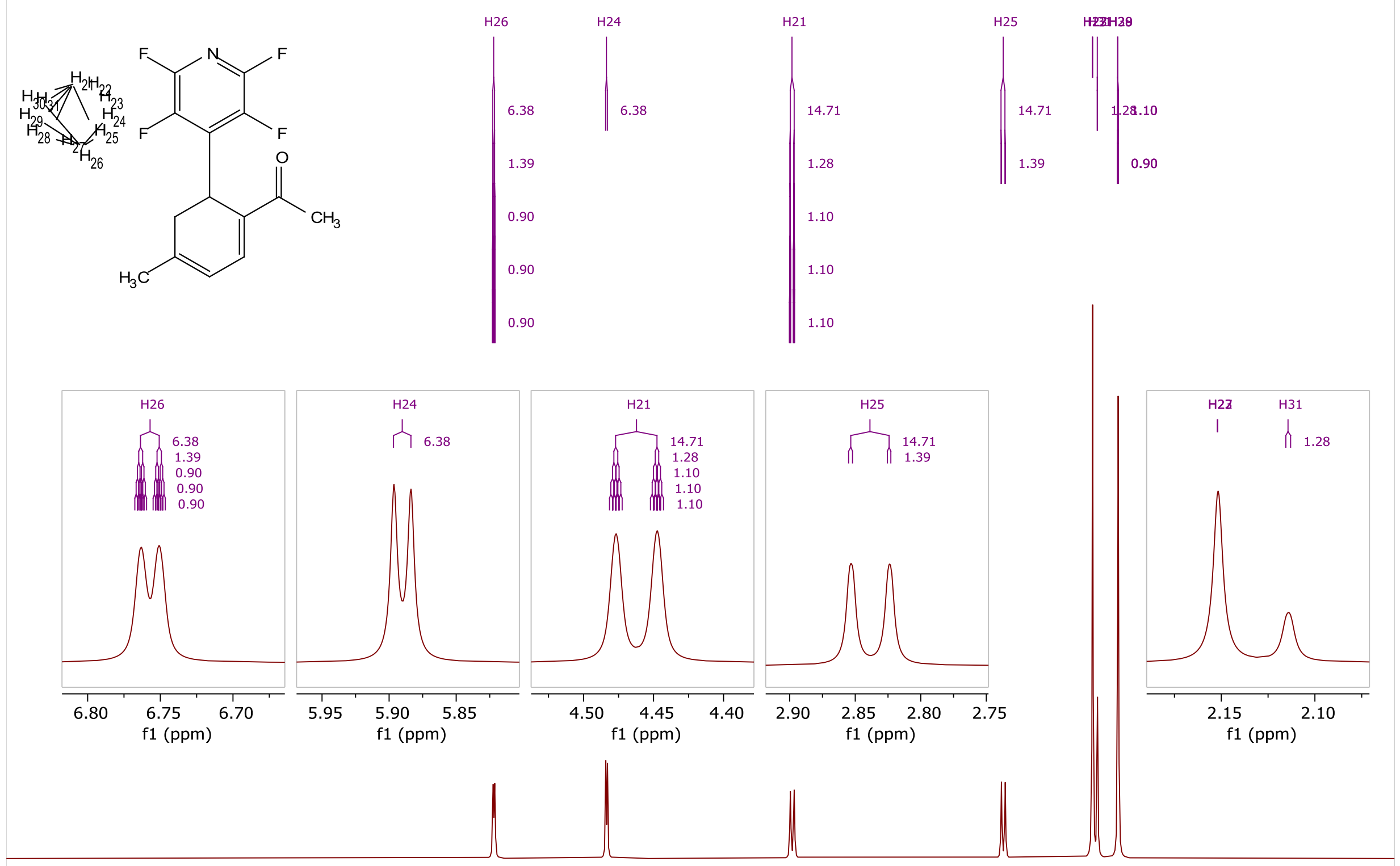

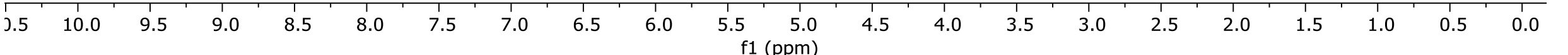


<smiles>CC(=O)C1=CCC(C)=CC1c1c(F)c(F)nc(F)c1F</smiles>

Diene Structure B
C

$\begin{array}{llll}\text { C } & 4.625901 & 1.423385 & -0.3422\end{array}$

$\begin{array}{llll}C & 5.729266 & 0.839051 & -0.96841\end{array}$

$\begin{array}{llll}\text { C } & 6.785255 & 1.637765 & -1.39841\end{array}$

$\begin{array}{llll}N & 6.810622 & 2.94543 & -1.25677\end{array}$

$\begin{array}{llll}\text { F } & 3.658906 & 3.477653 & 0.365667\end{array}$

$\begin{array}{llll}\text { F } & 7.832926 & 1.056553 & -1.99159\end{array}$

$\begin{array}{llll}\mathrm{F} & 5.788564 & -0.49103 & -1.16152\end{array}$

$\begin{array}{llll}\text { C } & 3.422108 & 0.612099 & 0.132987\end{array}$

$\begin{array}{llll}F & 5.803285 & 4.844896 & -0.53386\end{array}$

$\begin{array}{llll}\text { C } & 2.702027 & 0.01108 & -1.05509\end{array}$

$\begin{array}{llll}C & 2.627064 & -1.2972 & -1.31727\end{array}$

$\begin{array}{llll}C & 3.263163 & -2.31347 & -0.39875\end{array}$

$\begin{array}{llll}\text { C } & 3.735065 & -1.74483 & 0.905326\end{array}$

$\begin{array}{llll}\text { C } & 3.818945 & -0.43118 & 1.166235\end{array}$

$\begin{array}{llll}C & 4.275096 & 0.10206 & 2.483378\end{array}$

$\begin{array}{llll}\text { C } & 4.677056 & -0.85752 & 3.592946\end{array}$

$\begin{array}{llll}\mathrm{O} & 4.319923 & 1.311748 & 2.663839\end{array}$

$\begin{array}{llll}\text { C } & 1.920322 & -1.84366 & -2.52861\end{array}$

$\begin{array}{llll}H & 2.761634 & 1.332625 & 0.626897\end{array}$

$\begin{array}{llll}H & 2.242868 & 0.726347 & -1.73571\end{array}$

$\begin{array}{llll}H & 2.558934 & -3.13707 & -0.20163\end{array}$

$\begin{array}{llll}H & 4.114764 & -2.79432 & -0.9103\end{array}$

$\begin{array}{llll}H & 4.020735 & -2.46597 & 1.667851\end{array}$

$\begin{array}{llll}H & 5.512688 & -1.49564 & 3.283111\end{array}$

$\begin{array}{llll}H & 3.84523 & -1.51446 & 3.872002\end{array}$

$\begin{array}{llll}H & 4.977265 & -0.27004 & 4.462176\end{array}$

$\begin{array}{llll}H & 1.099069 & -2.51464 & -2.24125\end{array}$

$\begin{array}{llll}H & 1.505519 & -1.04375 & -3.1492\end{array}$

$\begin{array}{llll}H & 2.605792 & -2.4357 & -3.15084\end{array}$

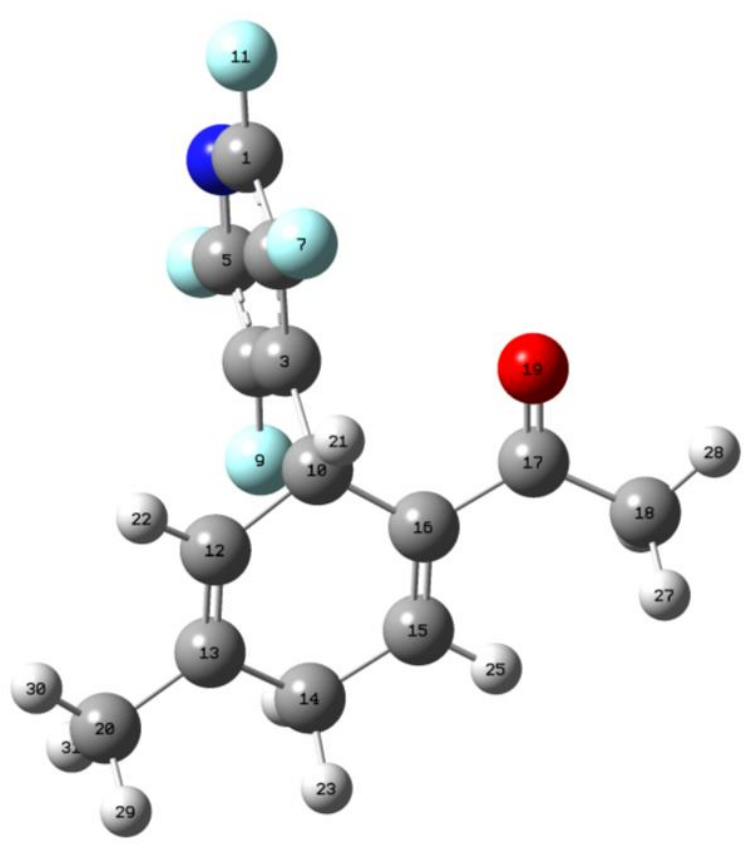


Computed diene spectrum B

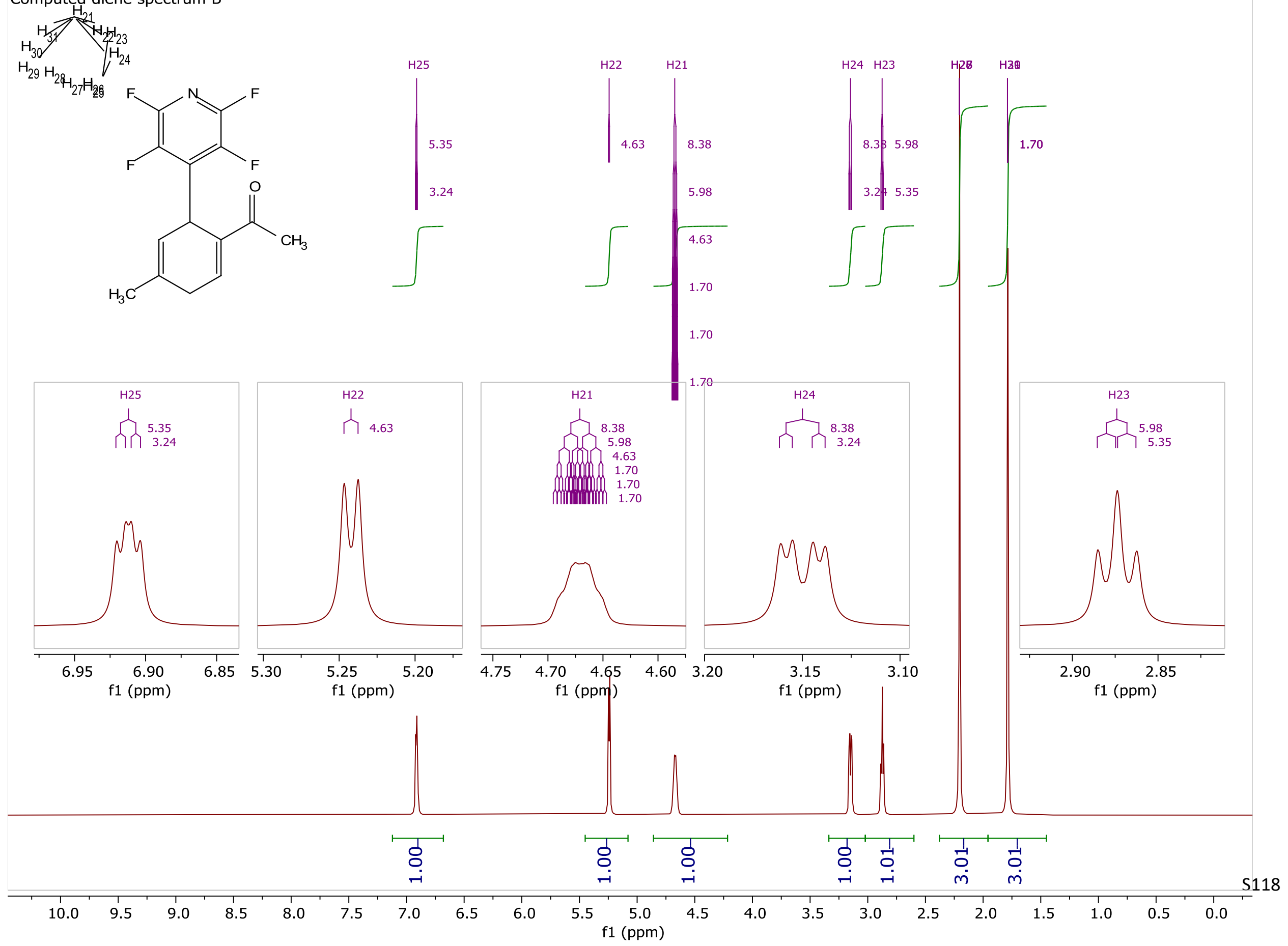


<smiles>CC(=O)C1=CC(c2c(F)c(F)nc(F)c2F)C(C)=CC1</smiles>

Diene Structure C
$\mathrm{H}$

H

$\mathrm{H}$

$\mathrm{H}$

$\mathrm{H}$

$\mathrm{H}$

$\mathrm{H}$

C

C

C

C

C

$$
\mathrm{F}
$$

C

$\mathrm{F}$

$\mathrm{F}$

\section{F}

c

C

\begin{tabular}{rrr}
3.245211 & 3.363823 & -0.58187 \\
2.057594 & -1.81474 & 4.457663 \\
2.722236 & -0.33298 & 3.722167 \\
3.688029 & -1.81398 & 3.736151 \\
4.158207 & -1.28067 & -3.66239 \\
5.664051 & -1.11332 & -2.73954 \\
4.700723 & 0.313189 & -3.11268 \\
4.273673 & -0.38182 & 1.887368 \\
0.9804 & -1.41767 & -0.28853 \\
1.714927 & -2.98 & -0.1193 \\
2.370684 & -2.14632 & -2.37917 \\
\hline 5.51141 & -0.53024 & -0.2486 \\
1.010358 & -2.51697 & 2.315185 \\
\hline 2.667713 & -1.42477 & 3.641216 \\
\hline 2.034789 & -1.84974 & 2.324768 \\
4.632218 & -0.74678 & -2.83345 \\
\hline 3.784337 & -0.71261 & 0.97444 \\
2.664178 & -1.44819 & 1.027903 \\
1.960584 & -1.91486 & -0.22059 \\
2.750928 & -1.67448 & -1.47385 \\
3.865355 & -0.94075 & -1.55117 \\
\hline 2.0926 & 4.032682 & -0.64854 \\
4.445663 & -0.27017 & -0.31199 \\
6.780871 & 1.42625 & -0.3821 \\
6.627914 & 4.116332 & -0.5623 \\
\hline .026889 & 1.344564 & -0.47253 \\
4.360052 & 4.063336 & -0.60468 \\
5.497647 & 3.407185 & -0.53843 \\
5.575358 & 2.018901 & -0.44525 \\
\hline .407033 & 1.254992 & -0.41858 \\
\hline & 1.973816 & -0.49131
\end{tabular}

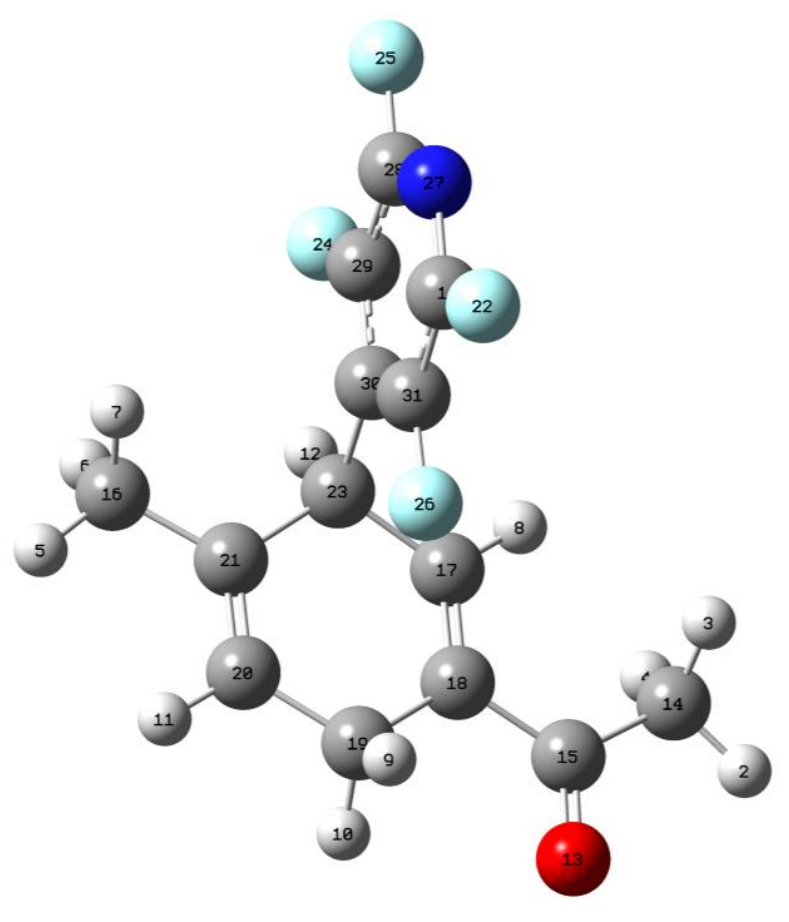


Computed diene spectrum $\mathrm{C}$
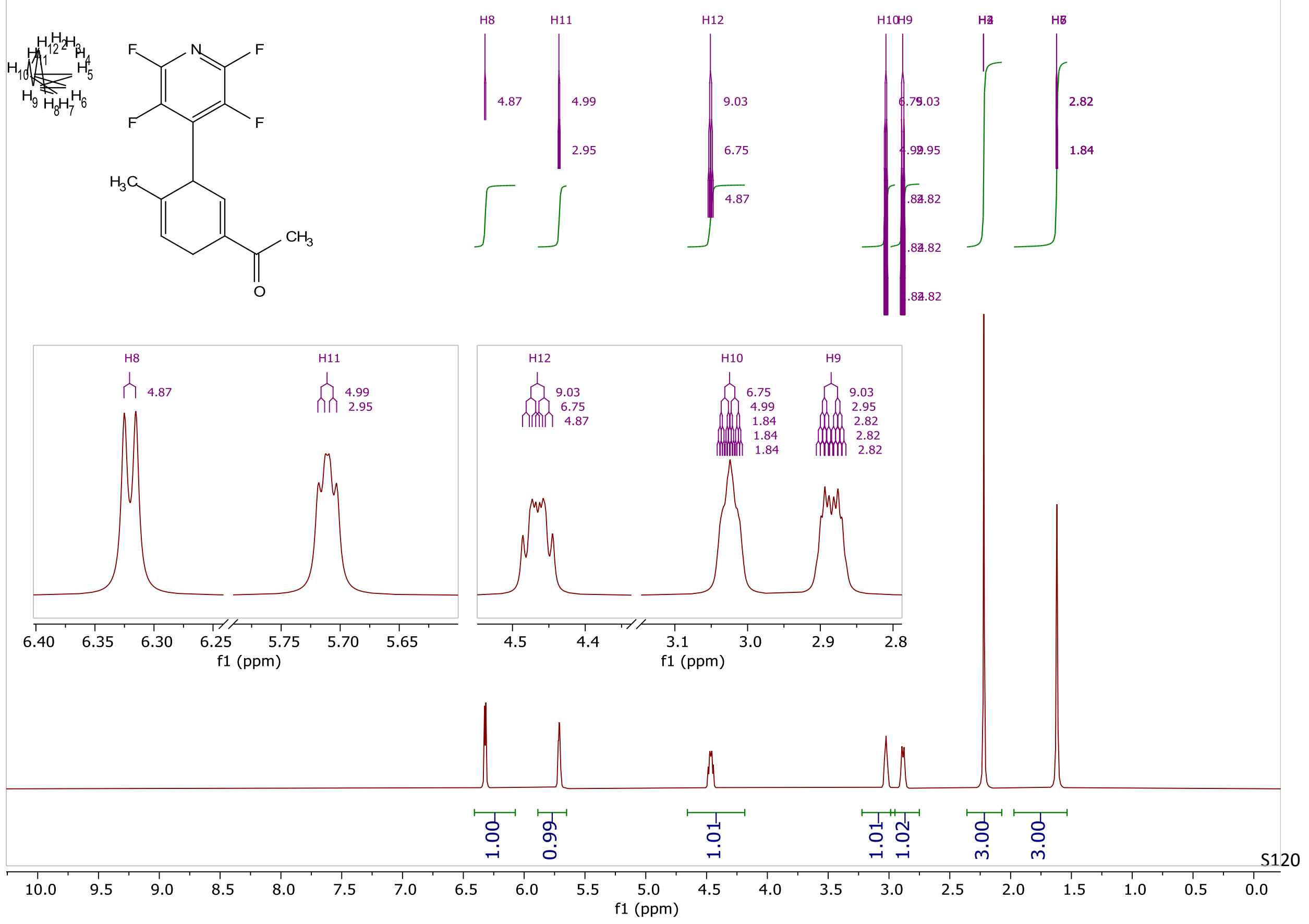


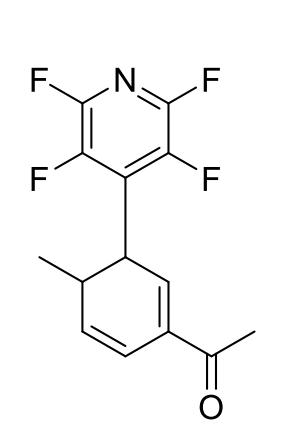

\begin{tabular}{|c|c|c|c|}
\hline $\mathrm{H}$ & 3.478092 & 0.704907 & 3.044957 \\
\hline H & 2.474395 & -0.74206 & 2.834845 \\
\hline r & 4.110976 & -0.88118 & 3.49941 \\
\hline ト & 5.774064 & -3.49023 & -2.52347 \\
\hline ト & 3.480614 & 0.438343 & -1.95935 \\
\hline r & 4.349214 & -2.7202 & 1.817034 \\
\hline ト & 4.118192 & -4.02681 & -2.81673 \\
\hline r & 5.075439 & -0.11119 & 1.321001 \\
\hline r & 4.342601 & -3.50513 & -0.48888 \\
\hline r & 5.07806 & -3.36263 & -4.16562 \\
\hline r & 2.166334 & -0.1788 & 0.404693 \\
\hline$c$ & 3.496735 & -0.3525 & 2.761997 \\
\hline c & 4.357966 & -1.87173 & -2.81301 \\
\hline$c$ & 4.051014 & -1.50223 & -1.38735 \\
\hline$C$ & 4.200513 & -2.01943 & 0.997938 \\
\hline c & 4.205979 & -1.06647 & -3.71805 \\
\hline C & 4.064966 & -0.5513 & 1.349735 \\
\hline $\mathrm{F}$ & 5.338939 & 4.571563 & 0.093153 \\
\hline $\mathrm{F}$ & 0.933514 & 1.691376 & 1.166031 \\
\hline C & 4.2055 & -2.44991 & -0.2725 \\
\hline$c$ & 4.865575 & -3.27439 & -3.09894 \\
\hline C & 3.611299 & -0.25359 & -1.13232 \\
\hline C & 3.204824 & 0.162525 & 0.265937 \\
\hline $\mathrm{F}$ & 5.461933 & 1.892156 & -0.25388 \\
\hline $\mathrm{F}$ & 1.011948 & 4.377135 & 1.443719 \\
\hline$N$ & 3.175213 & 4.46323 & 0.766391 \\
\hline C & 4.255753 & 3.836823 & 0.351001 \\
\hline C & 4.319907 & 2.457089 & 0.172662 \\
\hline C & 3.198039 & 1.667536 & 0.437304 \\
\hline C & 2.065777 & 2.355245 & 0.877635 \\
\hline C & 2.106627 & 3.740863 & 1.022647 \\
\hline
\end{tabular}


Computed diene spectrum $D$

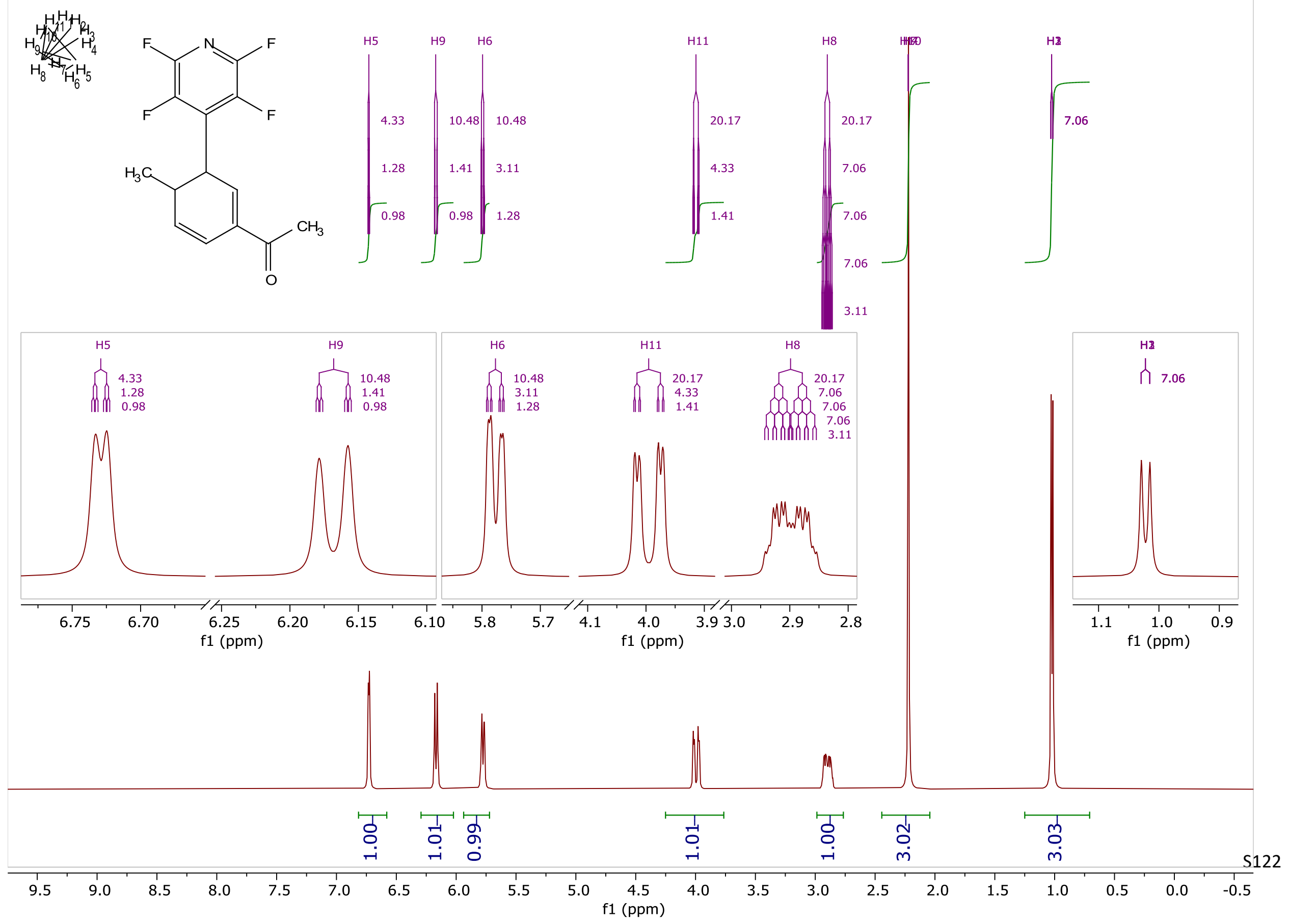



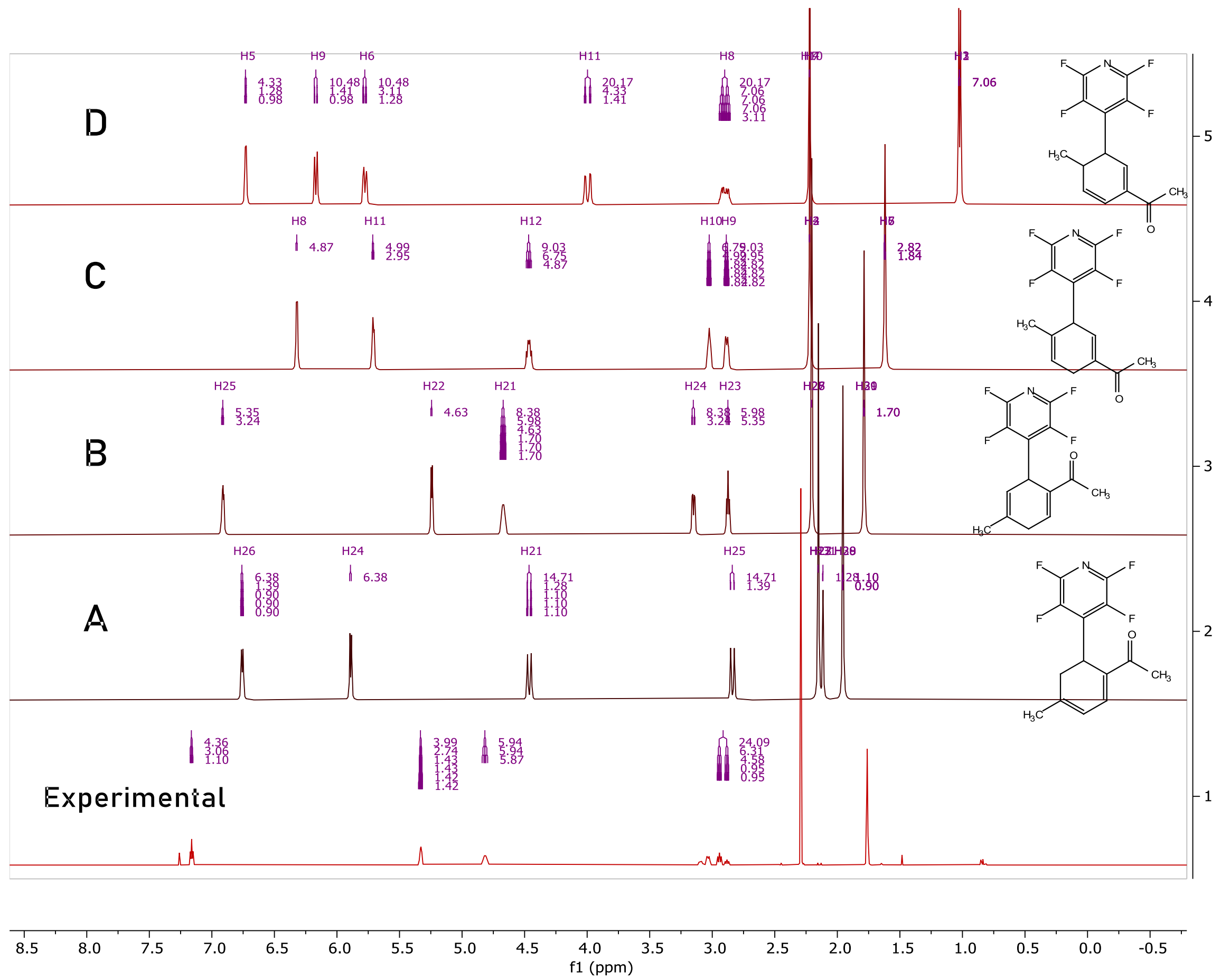


\begin{tabular}{|c|c|c|c|c|c|c|c|c|c|c|c|c|c|}
\hline \multirow[b]{2}{*}{$\begin{array}{c}\text { Experimental } \\
\text { shift }\end{array}$} & \multirow[b]{2}{*}{ Integral } & \multirow{2}{*}{$\begin{array}{c}\text { Computational } \\
\text { Shift: } \\
\text { Structure A }\end{array}$} & \multirow[b]{2}{*}{ Integral } & \multirow[b]{2}{*}{$\Delta$} & \multicolumn{2}{|l|}{ Computational } & \multirow[b]{2}{*}{$\Delta$} & \multirow{2}{*}{$\begin{array}{c}\text { Computational } \\
\text { Shift: } \\
\text { Structure C }\end{array}$} & \multirow[b]{2}{*}{ Integral } & \multicolumn{3}{|c|}{ Computational } & \multirow[b]{2}{*}{$\Delta$} \\
\hline & & & & & $\begin{array}{c}\text { Shift: } \\
\text { Structure B }\end{array}$ & Integral & & & & $\Delta$ & $\begin{array}{c}\text { Shift: } \\
\text { Structure D }\end{array}$ & Integral & \\
\hline 7.16 & 1 & 6.76 & 1 & 0.40 & 6.91 & 1 & 0.25 & 6.32 & 1 & 0.84 & 6.73 & 1 & 0.43 \\
\hline 5.33 & 1 & 5.89 & 1 & -0.56 & 5.24 & 1 & 0.09 & 5.71 & 1 & -0.38 & 6.17 & 1 & -0.84 \\
\hline 4.82 & 1 & 4.46 & 1 & 0.36 & 4.67 & 1 & 0.15 & 4.47 & 1 & 0.35 & 5.78 & 1 & -0.96 \\
\hline 3.06 & 1 & 2.84 & 1 & 0.22 & 3.15 & 1 & -0.09 & 3.02 & 1 & 0.04 & 3.99 & 1 & -0.93 \\
\hline 2.91 & 1 & 2.11 & 1 & 0.80 & 2.87 & 1 & 0.04 & 2.88 & 1 & 0.03 & 2.90 & 1 & 0.01 \\
\hline 2.29 & 3 & 2.15 & 3 & 0.14 & 2.20 & 3 & 0.09 & 2.22 & 3 & 0.07 & 2.22 & 3 & 0.07 \\
\hline 1.76 & 3 & 1.96 & 3 & -0.20 & 1.79 & 3 & -0.03 & 1.62 & 3 & 0.14 & 1.02 & 3 & 0.74 \\
\hline std dev & & & & 0.41 & & & 0.10 & & & 0.35 & & & 0.65 \\
\hline mean & & & & 0.17 & & & 0.07 & & & 0.15 & & & -0.21 \\
\hline
\end{tabular}




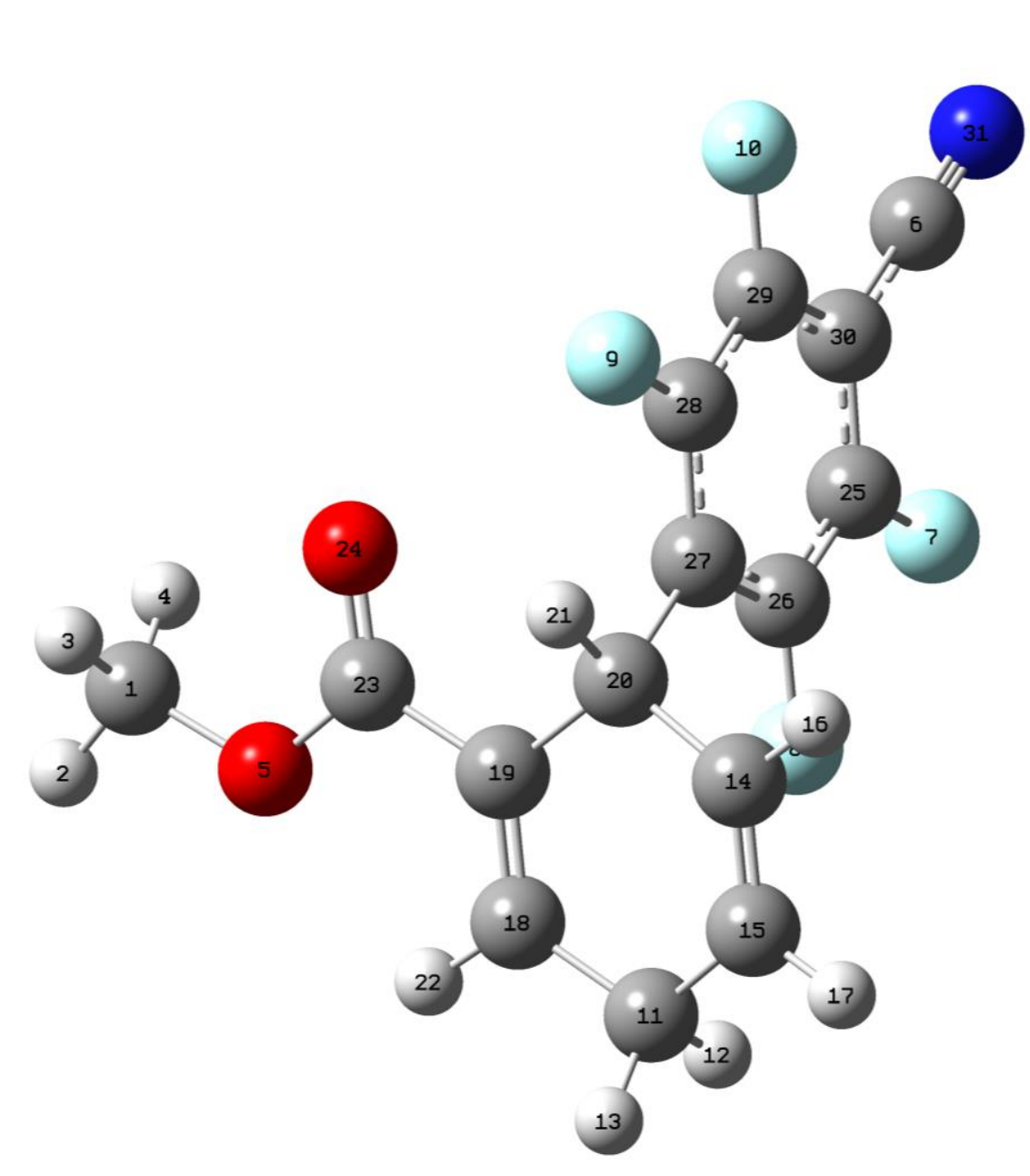

\section{4.192810714943}

$-0.819316164610$

4.137624952878

H 3.655127257694

$-1.331856343321$

H 5.262679481263

$-1.036927887829$

0.261739028424

H 4.0464514325

O 3.636201083005

C 2.923569184108

$-1.326261555225$

4.826675737268

F 0.917234325705

F 1.461793543937

2.884889470269

0.285295108197

F 6.105932152525

F 5.548688003209

1.257085469196

3.856859546273

C 2.004657199496

H 0.945074033085

$-2.906684136547$

H 1.975789781640

$-2.605514086352$

$-4.002161667699$

$-1.588289453755$

$-2.519307797476$

H 4.170638350493

$-1.340070665732$

H 2.441037809609

$-3.038836483290$

$-2.308463291131$

C 2.613631083428

C 3.566315579346

$-1.365484703016$

C 4.136195466954

H 5.228283051093

$-0.804342129281$

$-0.854453632853$

H 2.257117002069

C 4.166442031493

$-2.671770584095$

$-0.811495327757$

O 5.052172997302

C 2.193000828991

0.024352461626

2.492964250246

C 2.489200361368

C 3.806144658448

1.144820004424

0.679388399238

C 4.820518280424

C 4.542952890827

1.633574857927

2.985806023852

C 3.218783086482

$3.441712166198 \quad-1.262018944044$

4.935724697794

4.192217861469

4.207022499748

2.913932792117

$-1.431605591122$

$-1.202824555269$

$-0.890601332174$

$-1.002415025772$

$-1.309712507066$

$-0.669373379678$

$-0.730976546496$

$-0.565818393282$

$-1.967475294433$

$-1.930565026565$

$-2.905655421863$

$-2.843227373178$

0.563131544489

0.539569169546

$-0.754677789751$

$-0.689140334683$

1.522856295056

1.782703198819

1.783916905947

$-1.150169907753$

$-0.989152856446$

$-0.923581330272$

$-1.040027588556$

$-1.204054324725$ 


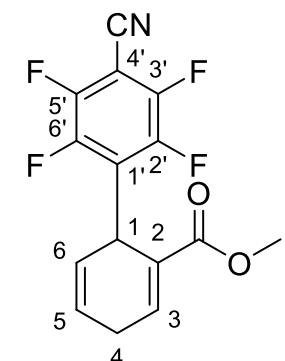

\begin{tabular}{ccc}
$\begin{array}{c}\text { Experimental shift } \\
(\mathrm{ppm})\end{array}$ & $\begin{array}{c}\text { Computational shift } \\
(\mathrm{ppm})\end{array}$ & $\begin{array}{c}\Delta \text { (EXP- } \\
\text { COMP) }\end{array}$ \\
\hline 7.25 & 7.19 & 0.06 \\
5.98 & 5.82 & 0.16 \\
5.69 & 5.5 & 0.19 \\
4.89 & 4.67 & 0.22 \\
3.63 & 3.6 & 0.03 \\
3.04 & 3.08 & -0.04
\end{tabular}

methyl 4'-cyano-2',3',5',6'-tetrafluoro-1,4-dihydro-[1,1'-biphenyl]-2-carboxylate 

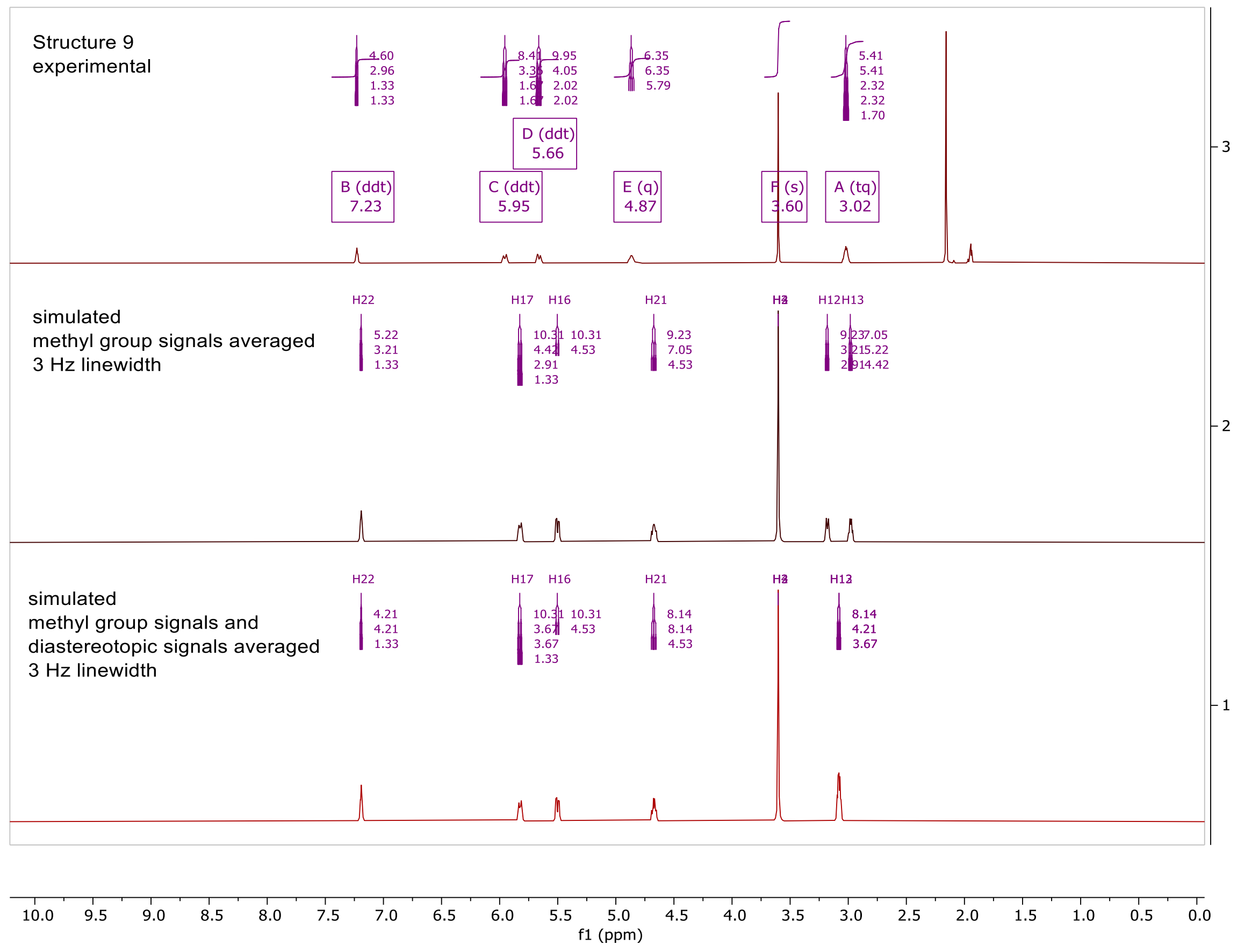


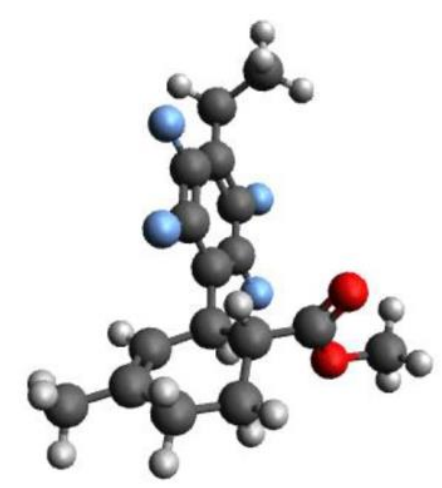<smiles>CCc1c(F)c(F)c(C2C=C(C)CCC2C(=O)O)c(F)c1F</smiles>

trans-Methyl-4'-ethyl-2',3', ', ',6'-tetrafluoro-5-methyl-1,2,3,4-tetrahydro-[1,1'- biphenyl]-2-carboxylate (28) model system

Basis set: B3LYP/6-31G* gas phase

Standard orientation (coordinates in Angstroms)

C 02.4250000 .3710001 .060000

C 01.0450000 .5170001 .183000

C $00.150000-0.2700000 .456000$

C $00.722000-1.214000-0.402000$

C $02.100000-1.357000-0.523000$

C $02.994000-0.5740000 .208000$

C $0-1.356000-0.0960000 .596000$

C $0-2.032000-1.3840001 .032000$

C $0-3.216000-1.8200000 .586000$

C $0-4.019000-1.036000-0.430000$

C $0-3.5310000 .409000-0.591000$

C $0-1.9940000 .458000-0.707000$

C $0-1.5080001 .861000-1.030000$

F 00.5820001 .4540002 .034000

C $0-3.818000-3.1200001 .054000$

F $0-0.070000-2.009000-1.148000$

F $02.581000-2.286000-1.373000$

F 03.2220001 .1710001 .797000

C $04.489000-0.7250000 .052000$

C $05.0380000 .012000-1.183000$

O $0-0.9820002 .196000-2.069000$
O $0-1.7530002 .719000-0.011000$

C $0-1.3230004 .073000-0.224000$

H $0-1.5050000 .6560001 .379000$

H 0 -1.489000 -1.973000 1.770000

H $0-3.981000-1.558000-1.399000$

H $0-5.079000-1.035000-0.139000$

H $0-3.9880000 .865000-1.478000$

H $0-3.8420001 .0080000 .274000$

H $0-1.682000-0.165000-1.550000$

H $0-3.164000-3.6400001 .761000$

H $0-4.789000-2.9560001 .541000$

H $0-4.004000-3.7930000 .205000$

H $04.977000-0.3480000 .956000$

H $04.729000-1.791000-0.026000$

H $06.122000-0.124000-1.258000$

H $04.581000-0.372000-2.100000$

H $04.8320001 .086000-1.122000$

H $0-1.5930004 .6120000 .684000$

H $0-0.2420004 .110000-0.386000$

H 0 - $1.8270004 .503000-1.094000$

Sum of electronic and thermal Free Energies = 1208.171102 Eh 


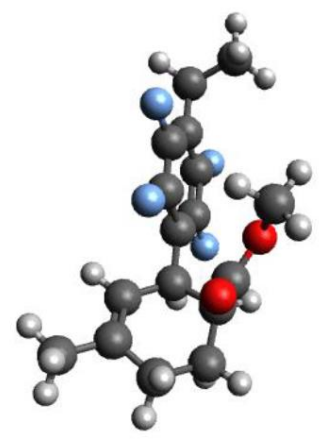<smiles>CCc1c(F)c(F)c(C2C=C(C)CCC2C(=O)OC)c(F)c1F</smiles>

cis-Methyl-4'-ethyl-2',3',5',6' -tetrafluoro-5-methyl-1,2,3,4-tetrahydro-[1,1'- biphenyl]-2-carboxylate (27) model system

Basis set: B3LYP/6-31G* gas phase

Standard orientation (coordinates in Angstroms)

C $0-2.545000-1.338000-0.354000$

C $0-1.188000-1.560000-0.576000$

C $0-0.198000-0.7690000 .010000$

C $0-0.6550000 .2620000 .834000$

C $0-2.0090000 .4840001 .053000$

C $0-2.997000-0.3130000 .474000$

C $01.273000-1.061000-0.238000$

C $02.090000-1.1220001 .039000$

C $03.403000-0.8720001 .108000$

C $04.204000-0.449000-0.102000$

C $03.433000-0.562000-1.428000$

C $01.954000-0.149000-1.315000$

C $01.8100001 .346000-1.052000$

F $0-0.840000-2.578000-1.389000$

C $04.163000-0.9660002 .405000$

F 00.2280001 .0950001 .419000

F $0-2.3740001 .5090001 .852000$

F $0-3.434000-2.151000-0.958000$

C $0-4.466000-0.0490000 .710000$

C $0-5.0120001 .104000-0.151000$

O $02.6480002 .072000-0.561000$
O $00.6080001 .797000-1.481000$

C $00.3430003 .185000-1.216000$

H $01.287000-2.062000-0.690000$

H $01.554000-1.4230001 .938000$

H $05.118000-1.057000-0.163000$

H 04.5340000 .5860000 .047000

H $03.454000-1.603000-1.777000$

H $03.9280000 .039000-2.199000$

H $01.444000-0.344000-2.265000$

H $04.637000-0.0050002 .650000$

H $04.971000-1.7070002 .340000$

H $03.511000-1.2440003 .240000$

H $0-4.6160000 .1880001 .769000$

H $0-5.026000-0.9650000 .501000$

H $0-4.8970000 .886000-1.218000$

H $0-4.4820002 .0370000 .067000$

H O -6.077000 1.2610000 .052000

H $01.1140003 .817000-1.663000$

H $00.3130003 .360000-0.138000$

H $0-0.6300003 .387000-1.665000$

Sum of electronic and thermal Free Energies = 1208.165044 Eh 
<smiles>CCCCCc1c(F)c(F)c([C@H]2C=C(C)CC[C@H]2C(=O)OC)c(F)c1F</smiles>

(27)

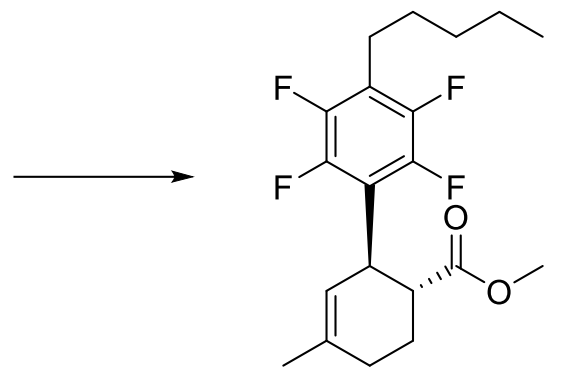

(28)

Addition of stoichiometric amounts of KHMDS in THF epimerizes the cis to the trans diastereomer.
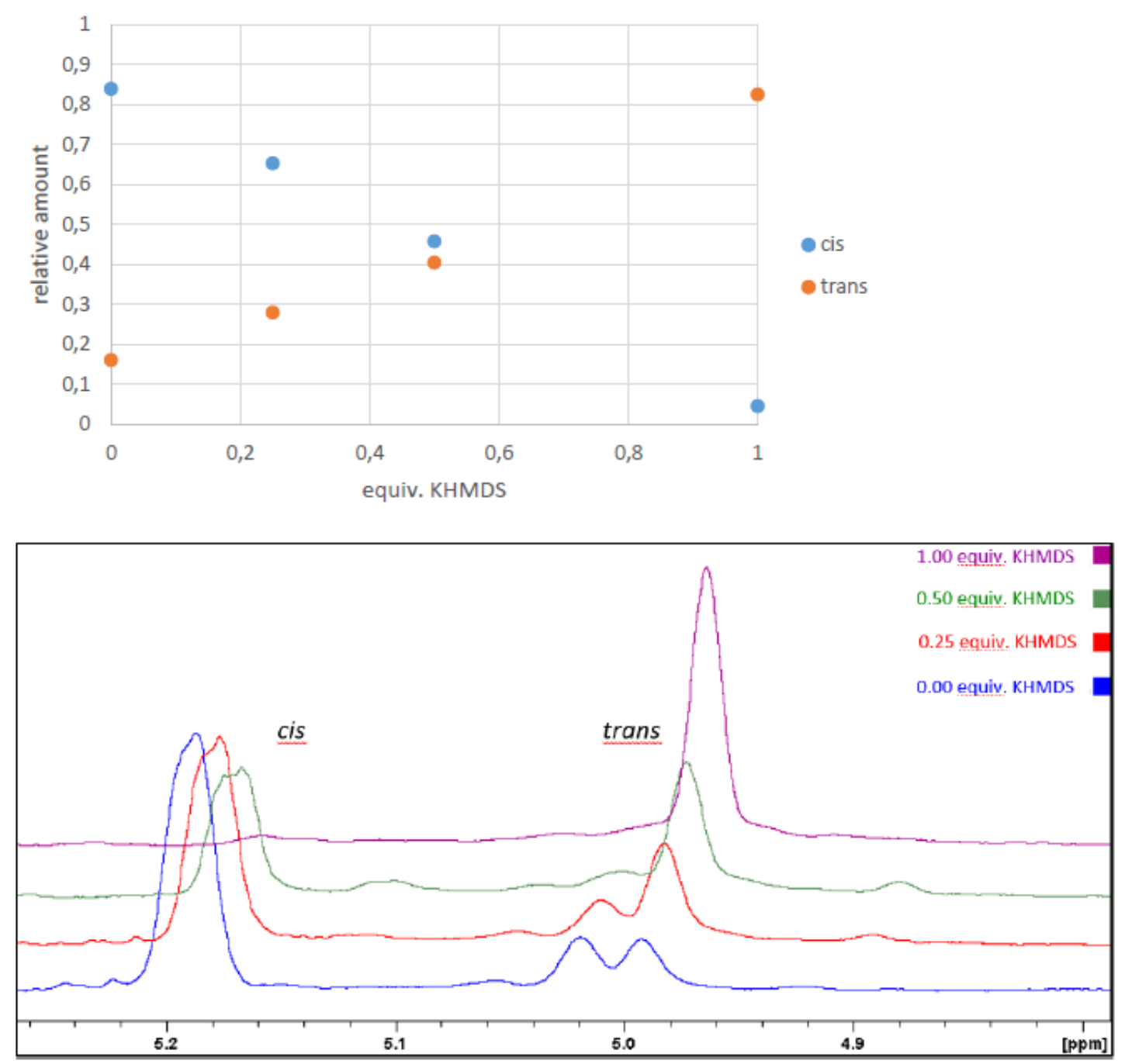

Epimerization of cis-(27) to trans-(28) (Top) Relative amounts determined via 1H NMR with THF (solvent) used as internal standard. (Bottom) 1H NMR showing the olefinic proton of cis and trans-28. Reaction conditions: SM (190 $\mu \mathrm{M}$ in THF) inside septum capped NMR tube. KHMDS (1M in THF) added stepwise. Values were corrected for dilution by additional THF from the KHMDS solution 


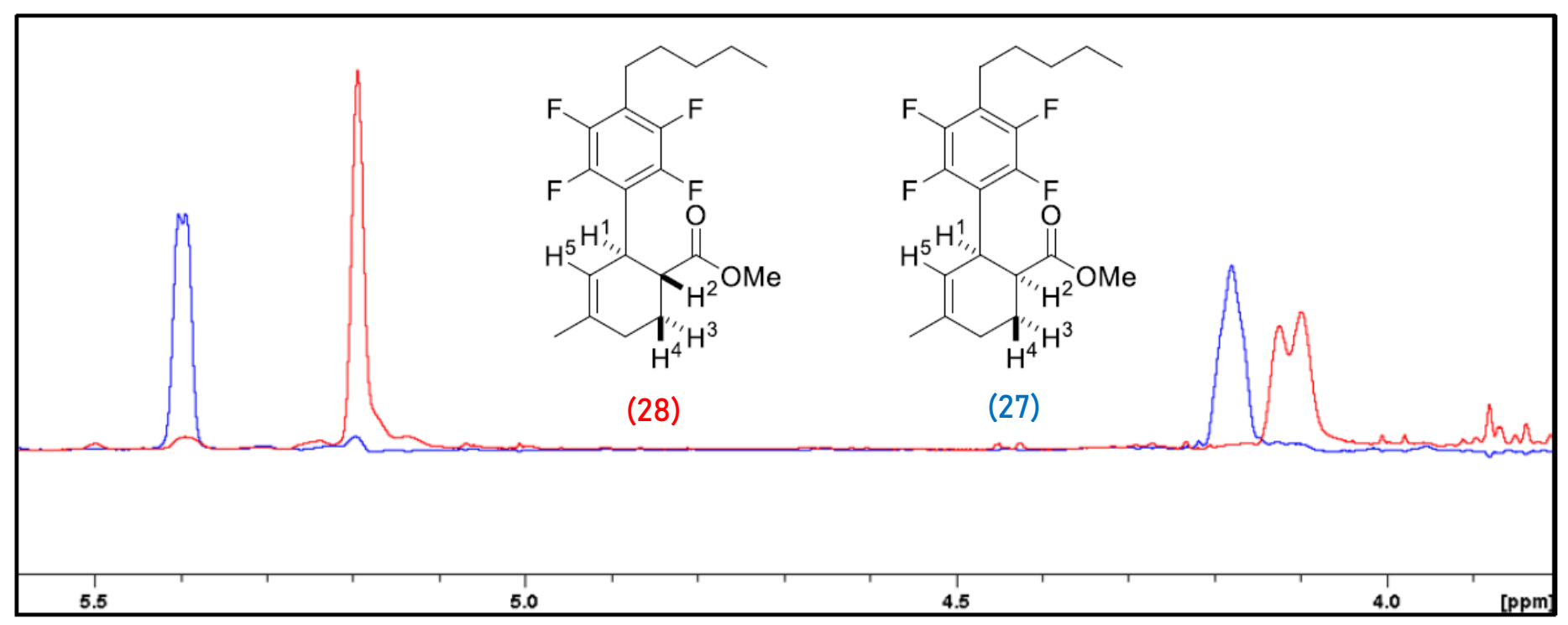

Superposition of the $1 \mathrm{H}$ NMR of (27) cis- and (28) trans-. Only the part showing the region of the olefinic (left) and benzylic (right) proton is depicted. 


\section{Single Crystal X-Ray Diffraction Analysis}

X-Ray analysis was performed by the crystallography laboratory of the University of Regensburg. Structure solving was done by Florian Meurer.

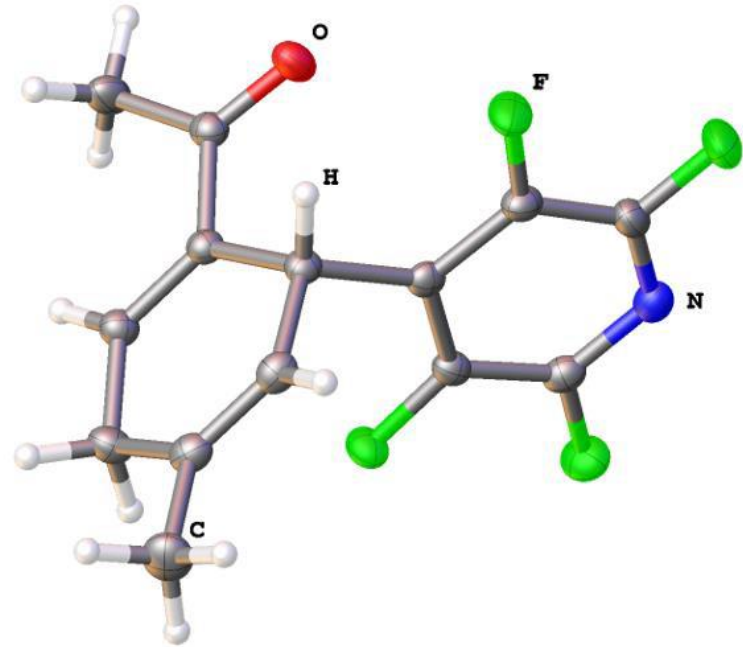

$\begin{array}{ll}\text { Formula } & \text { C14H11F4NO } \\ D_{\text {calc. } / \mathrm{g} \mathrm{cm}^{-3}} & 1.522 \\ \mu / \mathrm{mm}^{-1} & 1.201 \\ \text { Formula Weight } & 285.24 \\ \text { Colour } & \text { clear colourless } \\ \text { Shape } & \text { block } \\ \text { Size/mm } & 0.12 \times 0.11 \times 0.08 \\ T / \mathrm{K} & 123.00(10) \\ \text { Crystal System } & \text { monoclinic } \\ \text { Space Group } & 12 / a \\ a / \AA & 13.6681(2) \\ b / \AA & 10.05445(18) \\ c / \AA & 18.1356(3) \\ \left.\alpha\right|^{\circ} & 90 \\ \beta /^{\circ} & 93.0274(15) \\ \gamma /{ }^{\circ} & 90 \\ V / \AA 3 & 2488.81(7) \\ Z & 8 \\ Z^{\prime} & 1\end{array}$

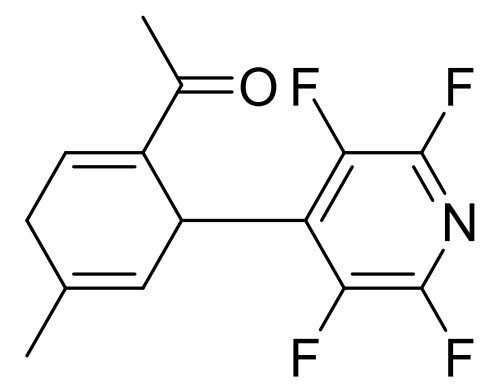

(8)

\begin{tabular}{|c|c|}
\hline Wavelength $/ \AA$ & 1.54184 \\
\hline Radiation type & CuK $\alpha$ \\
\hline$\Theta \min /{ }^{\circ}$ & 4.884 \\
\hline$\Theta \max /^{\circ}$ & 74.892 \\
\hline Measured Refl. & 16570 \\
\hline Independent Refl. & 2537 \\
\hline Reflections with I > 2(I) & 2290 \\
\hline Rint & 0.0265 \\
\hline Parameters & 183 \\
\hline Restraints & 0 \\
\hline Largest Peak & 0.257 \\
\hline Deepest Hole & -0.180 \\
\hline GooF & 1.036 \\
\hline wR2 (all data) & 0.0857 \\
\hline$w R 2$ & 0.0823 \\
\hline R1 (all data) & 0.0346 \\
\hline$R 1$ & 0.0307 \\
\hline
\end{tabular}

Fractional Atomic Coordinates $\left(\times 10_{4}\right)$ and Equivalent Isotropic Displacement Parameters $\left(\AA_{2} \times 10_{3}\right)$ for S046. Ueq is defined as $1 / 3$ of the trace of the orthogonalised $U_{i j}$.

\begin{tabular}{cllll}
\hline Atom & \multicolumn{1}{c}{$\mathbf{x}$} & $\mathbf{y}$ & $\mathbf{z}$ & $\boldsymbol{U}_{\text {eq }}$ \\
\hline F4 & $4022.5(5)$ & $5336.8(7)$ & $4642.3(4)$ & $25.04(18)$ \\
F3 & $4776.2(6)$ & $7046.6(8)$ & $5659.2(4)$ & $29.33(19)$ \\
F1 & $2591.8(6)$ & $9055.6(7)$ & $3400.2(4)$ & $31.4(2)$ \\
F2 & $3379.3(7)$ & $10571.8(8)$ & $4488.3(5)$ & $38.0(2)$ \\
O1 & $4227.7(7)$ & $7438.5(9)$ & $2537.6(5)$ & $26.7(2)$ \\
N1 & $4070.6(8)$ & $8804.2(11)$ & $5074.6(6)$ & $24.4(2)$ \\
C10 & $3832.0(8)$ & $6638.4(12)$ & $4565.8(6)$ & $18.9(2)$
\end{tabular}

$\begin{array}{cllll}\text { C9 } & 3269.9(8) & 7104.6(12) & 3959.2(6) & 18.4(2) \\ \text { C11 } & 4215.5(8) & 7516.6(13) & 5091.0(6) & 21.3(2) \\ \text { C3 } & 3646.0(8) & 5420.6(12) & 2990.1(6) & 17.8(2) \\ \text { C7 } & 2077.7(8) & 5280.8(13) & 3646.7(6) & 21.4(3) \\ \text { C4 } & 3734.9(8) & 4104.2(12) & 3054.5(6) & 20.2(2) \\ \text { C2 } & 4306.5(8) & 6226.0(12) & 2542.0(6) & 19.7(2) \\ \text { C8 } & 2844.8(8) & 6187.9(11) & 3353.1(6) & 18.3(2) \\ \text { C13 } & 3533.3(10) & 9257.4(12) & 4510.1(7) & 24.4(3) \\ \text { C6 } & 2164.2(9) & 3969.9(13) & 3706.0(6) & 22.2(3)\end{array}$




$\begin{array}{llllllllll}\text { C12 } & 3125.8(9) & 8471.9(12) & 3951.0(6) & 22.3(3) & \text { C1 } & 5048.8(10) & 5545.9(13) & 2087.3(7) & 26.9(3) \\ \text { C5 } & 3066.2(9) & 3258.1(12) & 3481.7(7) & 23.5(3) & \text { C14 } & 1373.7(10) & 3108.8(15) & 3996.9(8) & 32.5(3)\end{array}$

Anisotropic Displacement Parameters $\left(\times 10_{4}\right)$ S046. The anisotropic displacement factor exponent takes the form: -2 回 $2\left[h_{2} a^{*} \times U_{11}+\ldots\right.$ $\left.+2 h k a^{*} \times b^{*} \times U_{12}\right]$

\begin{tabular}{lcccrrr}
\hline Atom & $\boldsymbol{U}_{11}$ & $\boldsymbol{U}_{22}$ & $\boldsymbol{U}_{\mathbf{3 3}}$ & $\boldsymbol{U}_{23}$ & $\boldsymbol{U}_{\mathbf{1 3}}$ & $\boldsymbol{U}_{\boldsymbol{1 2}}$ \\
\hline F4 & $29.5(4)$ & $19.3(4)$ & $25.6(4)$ & $3.0(3)$ & $-5.0(3)$ & $4.5(3)$ \\
F3 & $29.0(4)$ & $33.2(4)$ & $24.8(4)$ & $2.3(3)$ & $-8.6(3)$ & $-1.1(3)$ \\
F1 & $45.0(5)$ & $22.9(4)$ & $25.5(4)$ & $2.5(3)$ & $-6.6(3)$ & $11.2(3)$ \\
F2 & $61.4(6)$ & $17.9(4)$ & $34.4(4)$ & $-2.8(3)$ & $-0.8(4)$ & $5.6(4)$ \\
O1 & $30.5(5)$ & $18.3(5)$ & $31.8(5)$ & $2.4(3)$ & $8.0(4)$ & $-0.9(3)$ \\
N1 & $26.8(5)$ & $25.1(6)$ & $21.6(5)$ & $-1.9(4)$ & $4.1(4)$ & $-3.6(4)$ \\
C10 & $17.5(5)$ & $18.5(6)$ & $20.9(5)$ & $2.4(4)$ & $3.5(4)$ & $1.4(4)$ \\
C9 & $16.1(5)$ & $21.1(6)$ & $18.4(5)$ & $0.9(4)$ & $4.6(4)$ & $1.0(4)$ \\
C11 & $18.2(5)$ & $26.7(6)$ & $19.0(5)$ & $2.4(5)$ & $1.7(4)$ & $-1.3(5)$ \\
C3 & $17.4(5)$ & $19.4(6)$ & $16.5(5)$ & $1.1(4)$ & $0.6(4)$ & $1.0(4)$ \\
C7 & $16.0(5)$ & $27.6(6)$ & $20.5(5)$ & $-1.2(5)$ & $1.4(4)$ & $0.6(4)$ \\
C4 & $19.4(5)$ & $20.0(6)$ & $21.5(5)$ & $1.7(4)$ & $1.9(4)$ & $1.6(4)$ \\
C2 & $20.7(5)$ & $19.7(6)$ & $18.6(5)$ & $1.6(4)$ & $0.0(4)$ & $-0.5(4)$ \\
C8 & $18.6(5)$ & $18.6(6)$ & $17.8(5)$ & $1.7(4)$ & $-0.1(4)$ & $2.8(4)$ \\
C13 & $32.2(6)$ & $17.1(6)$ & $24.6(6)$ & $-0.3(5)$ & $6.2(5)$ & $0.8(5)$ \\
C6 & $19.2(6)$ & $27.6(6)$ & $19.6(6)$ & $0.8(5)$ & $0.2(4)$ & $-3.1(5)$ \\
C12 & $25.4(6)$ & $22.3(6)$ & $19.2(5)$ & $3.1(4)$ & $2.1(4)$ & $4.7(5)$ \\
C5 & $23.2(6)$ & $19.7(6)$ & $27.7(6)$ & $4.6(5)$ & $2.2(5)$ & $-0.5(5)$ \\
C1 & $29.1(6)$ & $23.5(6)$ & $29.2(6)$ & $3.2(5)$ & $12.2(5)$ & $0.5(5)$ \\
C14 & $27.5(7)$ & $32.9(7)$ & $37.6(7)$ & $0.1(6)$ & $7.9(6)$ & $-8.4(6)$
\end{tabular}

Bond Lengths in $\AA$.

\begin{tabular}{lll}
\hline Atom & Atom & Length/Å \\
\hline F4 & C10 & $1.3401(14)$ \\
F3 & C11 & $1.3376(14)$ \\
F1 & C12 & $1.3408(14)$ \\
F2 & C13 & $1.3384(15)$ \\
O1 & C2 & $1.2239(15)$ \\
N1 & C11 & $1.3097(17)$ \\
N1 & C13 & $1.3097(17)$ \\
C10 & C9 & $1.3896(16)$ \\
C10 & C11 & $1.3816(17)$ \\
C9 & C8 & $1.5258(16)$ \\
C9 & C12 & $1.3888(17)$ \\
C3 & C4 & $1.3336(17)$ \\
C3 & C2 & $1.4861(16)$ \\
C3 & C8 & $1.5180(15)$ \\
C7 & C8 & $1.5078(16)$ \\
C7 & C6 & $1.3272(18)$ \\
C4 & C5 & $1.4947(16)$ \\
C2 & C1 & $1.5051(16)$ \\
C13 & C12 & $1.3793(18)$ \\
C6 & C5 & $1.5002(17)$ \\
C6 & C14 & $1.5018(17)$
\end{tabular}

Bond Angles in $\AA$.

\begin{tabular}{lllr}
\hline Atom & Atom & Atom & Angle $^{\circ}$ \\
\hline C11 & N1 & C13 & $116.08(11)$ \\
F4 & C10 & C9 & $120.51(11)$ \\
F4 & C10 & C11 & $119.22(10)$ \\
C11 & C10 & C9 & $120.26(11)$ \\
C10 & C9 & C8 & $122.70(10)$ \\
C12 & C9 & C10 & $114.49(11)$ \\
C12 & C9 & C8 & $122.81(10)$ \\
F3 & C11 & C10 & $119.11(11)$ \\
N1 & C11 & F3 & $116.52(11)$ \\
N1 & C11 & C10 & $124.37(11)$ \\
C4 & C3 & C2 & $122.24(10)$ \\
C4 & C3 & C8 & $122.07(10)$ \\
C2 & C3 & C8 & $115.67(10)$ \\
C6 & C7 & C8 & $124.66(11)$ \\
C3 & C4 & C5 & $123.70(11)$ \\
O1 & C2 & C3 & $119.38(11)$ \\
O1 & C2 & C1 & $120.70(11)$ \\
C3 & C2 & C1 & $119.90(10)$ \\
C3 & C8 & C9 & $111.31(9)$ \\
C7 & C8 & C9 & $110.88(9)$ \\
C7 & C8 & C3 & $112.13(10)$ \\
F2 & C13 & C12 & $119.13(11)$ \\
N1 & C13 & F2 & $116.58(11)$ \\
N1 & C13 & C12 & $124.29(12)$
\end{tabular}




$\begin{array}{llllllll}\text { C7 } & \text { C6 } & \text { C5 } & 121.52(11) & \text { F1 } & \text { C12 } & \text { C13 } & 118.67(11) \\ \text { C7 } & \text { C6 } & \text { C14 } & 122.57(12) & \text { C13 } & \text { C12 } & \text { C9 } & 120.49(11) \\ \text { C5 } & \text { C6 } & \text { C14 } & 115.92(11) & \text { C4 } & \text { C5 } & \text { C6 } & 113.76(10)\end{array}$

Hydrogen Fractional Atomic Coordinates $\left(\times 10_{4}\right)$ and Equivalent Isotropic Displacement Parameters $\left(\AA_{2} \times 10_{3}\right)$. Ueq is defined as $1 / 3$ of the trace of the orthogonalised $U_{i j}$.

\begin{tabular}{llllll}
\hline Atom & \multicolumn{1}{c}{$\mathbf{x}$} & $\mathbf{y}$ & \multicolumn{1}{c}{$\mathbf{z}$} & $\boldsymbol{U}_{\boldsymbol{e q}}$ \\
\hline H7 & 1503.07 & 5667.49 & 3797.01 & 26 \\
H4 & 4241.62 & 3689.32 & 2820.42 & 24 \\
H8 & 2519.41 & 6750.58 & 2973.05 & 22 \\
H5A & 3423.67 & 2934.21 & 3921.89 & 28 \\
H5B & 2869.87 & 2492.11 & 3184.22 & 28 \\
H1A & 4717.03 & 4990.19 & 1723.49 & 40 \\
H1B & 5474.97 & 5010.51 & 2403.05 & 40 \\
H1C & 5427.92 & 6203.12 & 1845.48 & 40 \\
H14A & 1627.89 & 2635.99 & 4425.23 & 49 \\
H14B & 1153.89 & 2484.33 & 3623.5 & 49 \\
H14C & 833.92 & 3655.67 & 4127.98 & 49
\end{tabular}




\section{References}

1. Fulmer, G. R.; Miller, A. J. M.; Sherden, N. H.; Gottlieb, H. E.; Nudelman, A.; Stoltz, B. M.; Bercaw, J. E.; Goldberg, K. I., NMR Chemical Shifts of Trace Impurities: Common Laboratory Solvents, Organics, and Gases in Deuterated Solvents Relevant to the Organometallic Chemist. Organometallics 2010, 29 (9), 2176-2179.

2. Lau, C. K.; Dufresne, C.; Belanger, P. C.; Pietre, S.; Scheigetz, J., Reductive deoxygenation of aryl aldehydes and ketones and benzylic, allylic, and tertiary alcohols by zinc iodide-sodium cyanoborohydride. The Journal of Organic Chemistry 1986, 51 (15), 3038-3043.

3. Bally, T.; Rablen, P. R., Quantum-Chemical Simulation of 1H NMR Spectra. 2. Comparison of DFT-Based Procedures for Computing Proton-Proton Coupling Constants in Organic Molecules. The Journal of Organic Chemistry 2011, 76 (12), 4818-4830. 